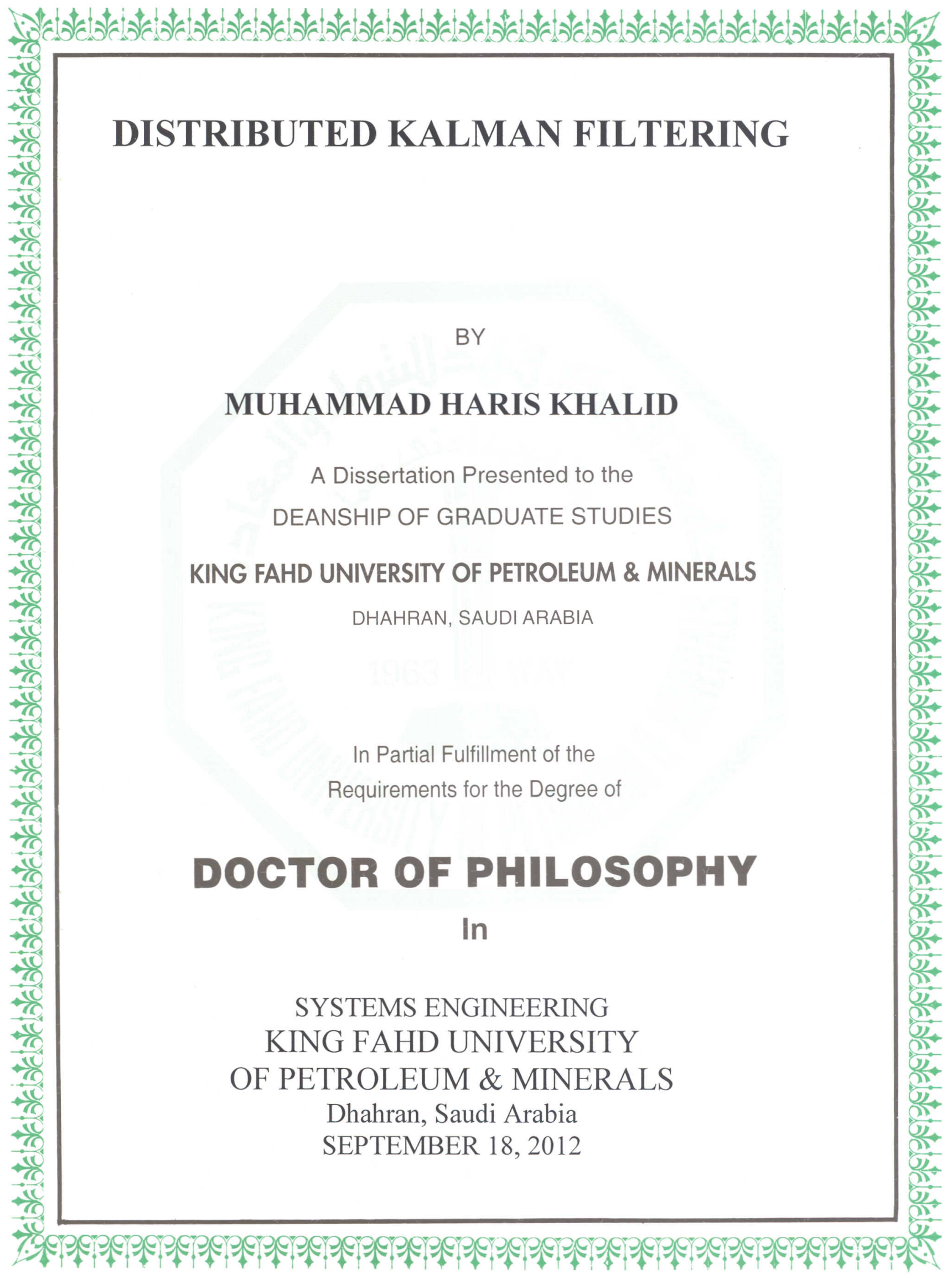




\section{KING FAHD UNIVERSITY OF PETROLEUM \& MINERALS \\ DHAHRAN- 31261, SAUDI ARABIA}

\section{DEANSHIP OF GRADUATE STUDIES}

This dissertation, written by MUHAMMAD HARIS KHALID under the direction of his dissertation advisor and approved by his dissertation committee, has been presented and accepted by the Dean of Graduate Studies, in partial fulfillment of the requirements for the degree of DOCTOR OF PHILOSOPHY IN SYSTEMS ENGINEERING.
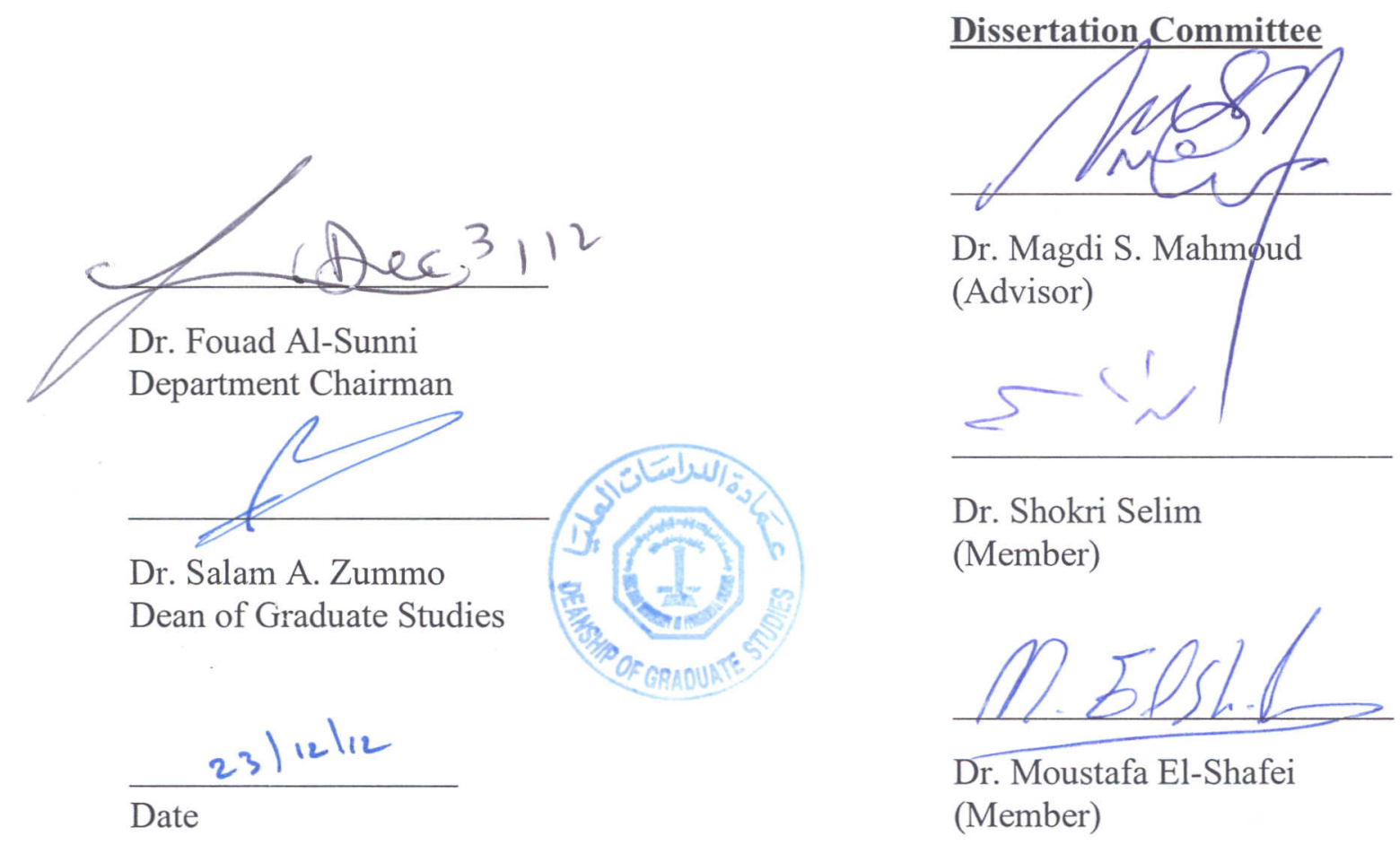

Dr. Shokri Selim (Member)

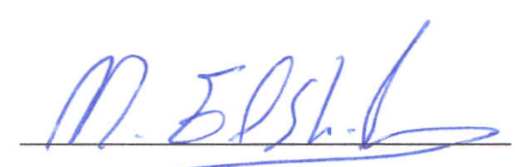

Dr. Moustafa El-Shafei (Member)

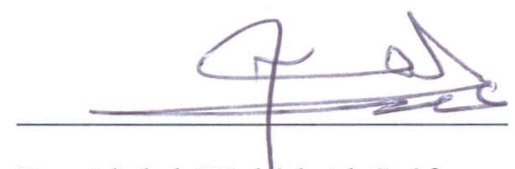

Dr. Abdul-Wahid Al-Saif (Member)

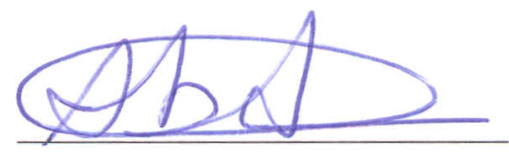

Dr. Mohammad Ali Abido (Member) 


\title{
DISTRIBUTED KALMAN FILTERING
}

by

\section{MUHAMMAD HARIS KHALID}

\author{
A Thesis Presented to the \\ DEANSHIP OF GRADUATE STUDIES
}

In Partial Fulfillment of the Requirements for the degree

DOCTOR OF PHILOSOPHY

IN

SYSTEMS ENGINEERING

KING FAHD UNIVERSITY

OF PETROLEUM \& MINERALS

Dhahran, Saudi Arabia

SEPTEMBER 18, 2012 
Dedicated to my loving Parents, Brother \& Sister 


\title{
ACKNOWLEDGMENTS
}

\author{
In the name of Allah, the Most Beneficent Most Merciful
}

With deep humility, I thank Almighty Allah, the merciful and beneficent, for his constantly reliable help and his everlasting inspiration. I would like to express my deepest thanks and appreciation to my dissertation advisor, Distinguished Professor Dr. Magdi S. Mahmoud, for his consistent help, guidance and attention that he devoted throughout the course of this work. His valuable suggestions and useful discussions to maintain the research quality made this work interesting to me. His support and words of encouragement gave a new life to my efforts in hard times. Without his help and valuable directions, this work would have never seen the light. I am equally grateful to my dissertation committee members Prof. Dr. Shokri Selim, Prof. Dr. Moustafa El-Shafei, Prof. Dr. Mohammad Ali Abido and Dr. Abdul-Wahid Al-Saif who have deeply guided me on the critical aspects of the work. I am also thankful to the Chairman of my Department, Prof. Dr. Fouad Al-Sunni who has been always supportive and provided enormous help in the support of this research. I wish to thank him from the depth of my heart.

I would also like to thank the Deanship of Scientific Research (DSR) at KFUPM for financial support through research group project RG-1105-1. The support and facilities 
provided by King Fahd University of Petroleum and Minerals for completing this work are highly appreciated. 


\section{TABLE OF CONTENTS}

LIST OF FIGURES ix

ABSTRACT (ENGLISH) - xii

$\begin{array}{ll}\text { ABSTRACT (ARABIC) } & \text { xV }\end{array}$

NOMENCLATURE Xvi

1 INTRODUCTION 1

1.1 Distributed Kalman Filtering . . . . . . . . . . . . . . . 1

1.2 Research Objectives and Methodology . . . . . . . . . . . . . . . . . 4

1.2.1 Problem Statement . . . . . . . . . . . . . . . 4

1.3 Dissertation Structure . . . . . . . . . . . . . . . . 5

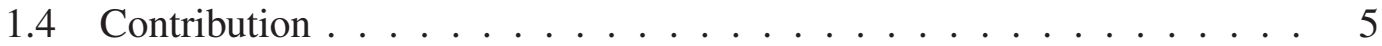

2 BIBLIOGRAPHIC REVIEW

2.1 An Overview ........................ 7

2.2 Introduction . . . . . . . . . . . . . . . 7

2.3 DKF Methods and their Applications . . . . . . . . . . . . . 11

2.3.1 DKF methods . . . . . . . . . . . . . . . . 11

2.3.2 DKF with applications . . . . . . . . . . . . 15

2.4 Diffusion-Based DKF . . . . . . . . . . . . . . . . . . . 18 
2.5 Distributed OOSM . . . . . . . . . . . . . . . . 22

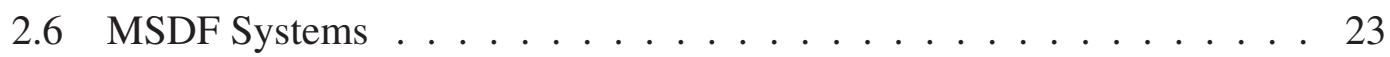

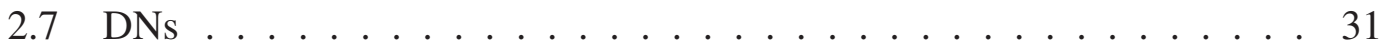

2.8 Mathematical Design in Track-to-Track Fusion $\ldots \ldots \ldots$. . . . 33

2.9 DC-Based Estimation . . . . . . . . . . . . . . . . . . . 36

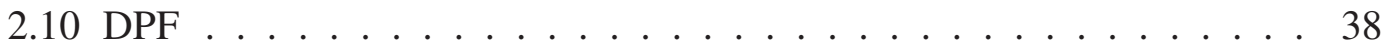

2.11 ST-Based Distributed Fusion Kalman Filter . . . . . . . . . . . 41

3 APPROXIMATE DISTRIBUTED ESTIMATION 44

3.1 An Overview . . . . . . . . . . . . . . . . . . . . . . . . . 44

3.2 Introduction $\ldots \ldots \ldots \ldots \ldots \ldots \ldots \ldots \ldots \ldots$

3.3 Problem Formulation $\ldots \ldots \ldots \ldots$

3.4 Bayesian-based FB Kalman Filter Fusion with Complete Prior Infor-

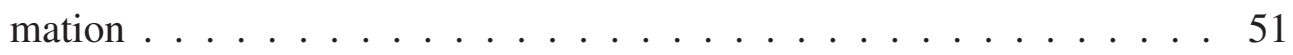

3.4.1 Modified Filter with Complete Prior Information . . . . . . 54

3.4.2 Approximating the Filter for Complete Prior Information . . . . 57

3.5 Bayesian-Based FB Kalman Filter Fusion without Prior Information . . 62 3.5.1 Modified Kalman Filter Without Prior Information . . . . . . 64

3.5.2 Approximating the Kalman Filter for Without Prior Information 65

3.6 Bayesian-Based FB Kalman Filter Fusion with Incomplete Prior Infor-

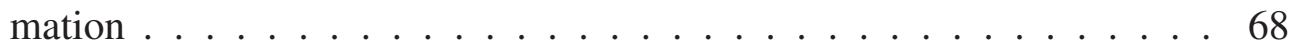

3.6.1 Modified Kalman Filter With Incomplete Prior Information . . . 70

3.6.2 Approximating the Kalman Filter for Incomplete Prior Infor-

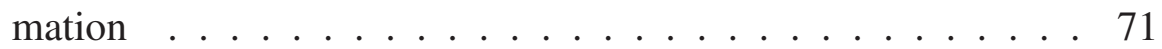

3.7 Fusion Algorithm . . . . . . . . . . . . . . . . . . . . . . . 74

3.8 Evaluation and Testing $\ldots \ldots \ldots \ldots \ldots$

3.8.1 Evaluation of results . . . . . . . . . . . . . 79

3.8 .2 Time Computation . . . . . . . . . . . . . 83

4 A DISTRIBUTED EM-BASED KALMAN SMOOTHER 95

4.1 An Overview . . . . . . . . . . . . . . . . . . . . . . . . 95 
4.2 Introduction . . . . . . . . . . . . . . . . . . . . 95

4.3 Problem Formulation . . . . . . . . . . . . . . . . . 98

4.4 Full-Order EM-Based Fusion Smoothers . . . . . . . . . . . . . . . 100

4.4.1 Computation of cross covariance . . . . . . . . . . . 104

4.4.2 Estimation of the model parameters using an EM algorithm . 107

4.4 .3 Full-Order Fusion $\ldots \ldots 111$

4.5 Reduced-Order EM-Based SEEK Smoother . . . . . . . . . . . . . . 114

4.5.1 SEEK Smoother: A Reduced-Order Kalman . . . . . . . . . 114

4.5.2 Estimation of the model parameters using an EM algorithm . . 120

4.5.3 Reduced-Order Fusion . . . . . . . . . . . . . . . . . . . . . . 123

4.6 Evaluation and Testing . . . . . . . . . . . . . 125

4.6.1 Description of the Power Quality Lab . . . . . . . . . 125

4.6 .2 Load Scenarios . . . . . . . . . . . . . . . . . 127

4.6 .3 Evaluation of results $\ldots \ldots \ldots \ldots . \ldots 128$

\section{DISTRIBUTED ESTIMATION VIA INFORMATION MATRIX APPROACH140}

5.1 An Overview . . . . . . . . . . . . . . . . . . . . . 140

5.2 Introduction . . . . . . . . . . . . . . . . . 140

5.3 Problem Formulation . . . . . . . . . . . . . . . . . . . . 142

5.4 Covariance Intersection . . . . . . . . . . . . . . . . . 144

5.4.1 Information-Based Covariance Intersection Filter Algorithm . 145

5.4.2 Information-Based Covariance Intersection Filter: Complete Feedback Case . . . . . . . . . . . . . . . . . 147

5.4.3 Information-Based Covariance Intersection Filter: Partial Feedback Case . . . . . . . . . . . . . . . . . 151

5.5 Weighted Covariance . . . . . . . . . . . . . . 152

5.5.1 Information-Based Weighted Covariance Filter Algorithm . . 154

5.5.2 Information-Based Weighted Covariance Filter: Complete Feedback Case . . . . . . . . . . . . . . . 156 
5.5.3 Information-Based Weighted Covariance Filter: Partial Feedback Case . . . . . . . . . . . . . . . . . . 160

5.6 Kalman-Like Particle Filter . . . . . . . . . . . . . . . . . . . . . 161

5.6.1 Information-Based Kalman-Like Particle Filter Algorithm . . 163

5.6.2 Information-Based Kalman-Like Particle Filter: Complete Feedback Case . . . . . . . . . . . . . . . . 165

5.6.3 Information-Based Kalman-Like Particle Filter: Partial Feedback Case . . . . . . . . . . . . . . . . . . . 168

5.7 Measurement Fusion Algorithm . . . . . . . . . . . . . . . . . 169

5.8 On Functional Equivalence of two measurement Fusion Methods . . . . 171

5.9 Evaluation and Testing . . . . . . . . . . . . . . . 180

5.9 .1 Utility boiler . . . . . . . . . . . . . . . . . 180

5.9 .2 Evaluation of results $\ldots \ldots \ldots \ldots$. . . . . . . 182

6 CONCLUSIONS AND PERSPECTIVES 192

6.1 Research Conclusions . . . . . . . . . . . . . . . . . . . . 192

6.2 Future Research Work . . . . . . . . . . . . . . . . . . . . . . 194

$\begin{array}{ll}\text { REFERENCES } & 196\end{array}$

$\begin{array}{ll}\text { VITAE } & 255\end{array}$ 


\section{LIST OF FIGURES}

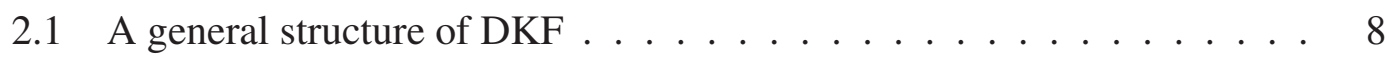

2.2 Classification of Distributed Kalman Filter* . . . . . . . . . . 10

3.1 Proposed Data Fusion Design . . . . . . . . . . . . . . . . . . . . 74

3.2 Architecture of LTIP in distributed control network . . . . . . . . . 77

3.3 Comparison of Covariance for complete prior information for Controller Fault . . . . . . . . . . . . . . . . . . . . . . . . . . . 84

3.4 Comparison of Estimates for complete prior information for Leakage Fault . . . . . . . . . . . . . . . . . . . . . . . . . 84

3.5 Comparison of Estimates for complete prior information for Controller

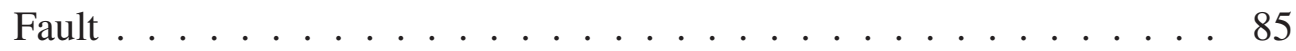

3.6 Comparison of Covariance for complete prior information for Leakage Fault with Upper Bound Modified Filter . . . . . . . . . . . . 85

3.7 Comparison of Covariance for complete prior information for Controller Fault with Upper Bound Modified Filter . . . . . . . . 86

3.8 Comparison of Covariance for complete prior information for Controller Fault with Lower Bound Modified Filter . . . . . . . . . 86

3.9 Comparison of Estimates for complete prior information for Leakage Fault with Lower Bound Modified Filter . . . . . . . . . . . . . . 87

3.10 Comparison of Estimates for complete prior information for Controller Fault with Lower Bound Modified Filter . . . . . . . . . . . . . 87

3.11 Comparison of Estimates for Incomplete prior information for Con-

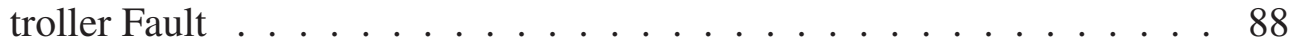


3.12 Comparison of Covariance for Incomplete prior information for Leakage Fault with Upper Bound Modified Filter . . . . . . . . . . . . 88

3.13 Comparison of Covariance for without prior information for Leakage Fault . . . . . . . . . . . . . . . . . . . . . 89

3.14 Comparison of Covariance for without prior information for Controller Fault . . . . . . . . . . . . . . . . . . . . 89

3.15 Comparison of Estimates for without prior information for Controller

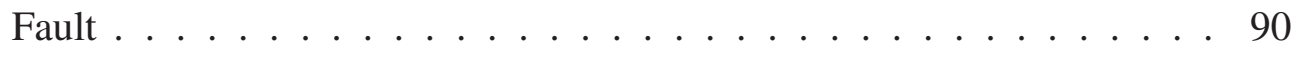

3.16 Comparison of Covariance for without prior information for Leakage Fault with Upper Bound Modified Filter . . . . . . . . . . . . . 990

3.17 Comparison of Covariance for without prior information for Controller Fault with Upper Bound Modified Filter . . . . . . . . . . . . . . . . 91

3.18 Comparison of Covariance for without prior information for Leakage Fault with Lower Bound Modified Filter . . . . . . . . . . . . . 91

3.19 Comparison of Covariance for without prior information for Controller Fault with Lower Bound Modified Filter . . . . . . . . . . . . . . 92

3.20 Comparison of Estimates for without prior information for Leakage Fault with Lower Bound Modified Filter . . . . . . . . . . . . . . 92

3.21 Comparison of Estimates for without prior information for Controller Fault with Lower Bound Modified Filter . . . . . . . . . . . . . 93

4.1 Estimates of Kalman-like particle and Basic Kalman smoother . . . . . 101

4.2 Mean Square Error: Kalman-like particle and basic Kalman smoother . 101

4.3 Power System Lab . . . . . . . . . . . . . . . . . . . . . . 127

4.4 Estimates for full-order smoother for Phase 3: DC motor drive Load . . 132

4.5 Estimates for full-order smoother for Phase 3: Linear Load . . . . . . 132

4.6 Estimates for full-order smoother for Phase 3: Nonlinear Load . . . . 133

4.7 Estimates for reduced-order smoother for Phase 1: DC motor drive Load 133

4.8 Estimates for reduced-order smoother for Phase 2: DC motor drive Load 134

4.9 Estimates for reduced-order smoother for Phase 3: DC motor drive Load 134 
4.10 Estimates for various reduced-order smoothers: DC motor Load . . . . 135

4.11 Estimates for reduced-order smoother for Phase 1: Linear Load . . . . . 135

4.12 Estimates for reduced-order smoother for Phase 2: Linear Load . . . . . 136

4.13 Estimates for reduced-order smoother for Phase 3: Linear Load . . . . . 136

4.14 Estimates for various reduced-order smoothers: Linear Load . . . . . . 137

4.15 Estimates for reduced-order smoother for Phase 1: Nonlinear Load . . . 137

4.16 Estimates for reduced-order smoother for Phase 2: Nonlinear Load . . . 138

4.17 Estimates for reduced-order smoother for Phase 3: Nonlinear Load . . . 138

4.18 Estimates for various reduced-order smoothers: Nonlinear Load . . . 139

4.19 Mean Square Error: EM-Based full and reduced-order smoothers . . . . 139

5.1 Estimates of Kalman-like particle and Basic Kalman filter . . . . . . . 162

5.2 Mean Square Error: Kalman-like particle and basic Kalman filter . . . . 162

5.3 Measurement fusion employed for information-based sensor . . . . . 170

5.4 State vector fusion employed for information-based sensor . . . . . . 170

5.5 Schematic Diagram of an Industrial Utility Boiler . . . . . . . . . 181

5.6 Covariance Intersection: Complete Feedback Comparison . . . . . . . . 186

5.7 Covariance Intersection: Partial Feedback Comparison ..... . 186

5.8 Covariance Intersection: No Feedback Comparison . . . . . . . . . . . 187

5.9 Covariance Intersection: Feedback Comparison _ . . . . . . . . . 187

5.10 Weighted Covariance: Complete Feedback Comparison . . . . . . . . 188

5.11 Weighted Covariance: Partial Feedback Comparison . . . . . . . . 188

5.12 Weighted Covariance: Feedback Comparison . . . . . . . . . . . 189

5.13 Kalman-like Particle Filter: Complete Feedback Comparison . . . . . . 189

5.14 Kalman-like Particle Filter: Partial Feedback Comparison . . . . . . . . 190

5.15 Kalman-like Particle Filter: Feedback Comparison . . . . . . . . . 190

5.16 Kalman-like Particle Filter: Complete Feedback with measurement fusion method ........................ 191 


\section{THESIS ABSTRACT}

NAME: $\quad$ Muhammad Haris Khalid

TITLE OF STUDY: Distributed Kalman Filtering

MAJOR FIELD: $\quad$ Systems Engineering

DATE OF DEGREE: September 18, 2012

In recent years, a compelling need has arisen to understand the effects of distributed information structures on estimation and filtering. In this thesis, distributed Kalman filtering has been on focus with various perspectives. Firstly, a bibliographical review on distributed Kalman filtering $(D K F)$ is provided. A classification of different approaches and methods involved to DKF has been elaborated, followed by the applications of DKF are also discussed and explained separately. A comparison of different approaches is briefly carried out. Focuses on the contemporary research are also addressed with emphasis on the practical application of the techniques. An exhaustive list of publications, linked directly or indirectly to DKF in the open literature, is compiled to provide an overall picture of different developing aspects of this area. 
Secondly, an approximate distributed estimation within distributed networked control formalism has been proposed. This is made possible by using Bayesian-based forward-backward (FB) system with generalized versions of Kalman filter. The analytical treatment is presented for cases with complete, incomplete or no prior information with bounds and then followed by estimation fusion for all three cases. The proposed scheme is validated on a rotational drive-based electro-hydraulic system and the ensuing results ensured the effectiveness of the scheme underpinning it.

The thesis proposes distributed expectation maximization(EM)-based reduced-order singular evolutive extended Kalman (SEEK) smoother. Optimal reduced-order smoothers complement the computation by doing re-analysis to correct the state of a dynamic system. The nature of order reduction of the SEEK smoother is fulfilling this phase, and made more precise by injecting the Kalman-like particle nature of the filter. The proposed scheme is first evaluated with its distributed full-order EM-based smoother version, followed by its reduced order version. The EM algorithm plays its role to identify and improve the estimate of process noise covariance $Q$ in each case. The proposed scheme is then validated on a power quality system with various kinds of loads, ensuring the effectiveness and applicability of the scheme underpinning it.

An approach for distributed estimation algorithm is proposed using information matrix filter on a distributed tracking system in which $N$ number of sensors are tracking the same target. The approach incorporates proposed engineered versions of information matrix filter derived from covariance intersection, weighted covariance and Kalman-like particle filter $(K L P F)$ respectively. The steady performance of these filters 
is evaluated with different feedback strategies, moreover employing them with commonly used measurement fusion methods i.e. measurement fusion and state-vector fusion respectively to complete the picture. The proposed filters are then validated on an industrial utility boiler, ensuring the effectiveness and applicability of the scheme underpinning it.

Keywords: $D K F$, Bayesian approach, prior information, distributed estimation, approximate estimation, electro-hydraulic system, expectation maximization, power system quality, EM smoother, information matrix filter, covariance intersection, weighted covariance, KLPF, industrial utility boiler. 


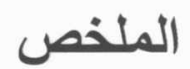

تزايدت فى الآونه الاخيرة الحاجه الملحه لاستيعاب التاثيرات المتعددة لهياكل

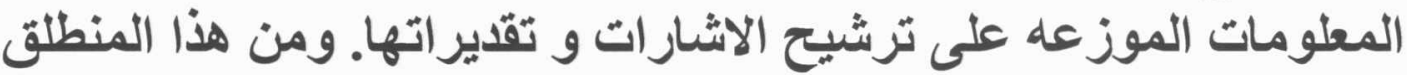

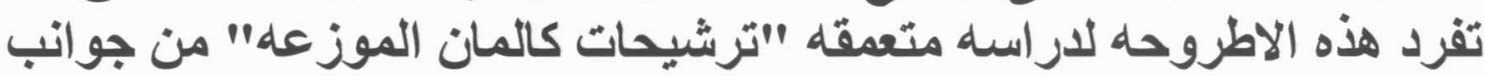

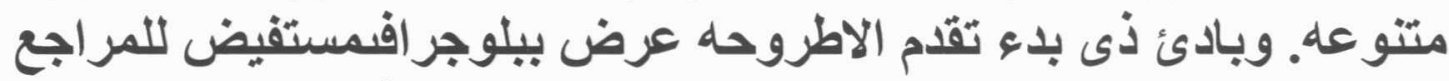

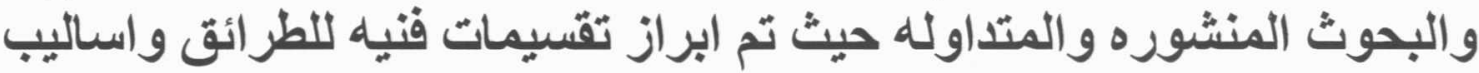
البحث ثم استعرضت التطبيقات المتنوعة وأجرت دراسه مقارنه متعدده

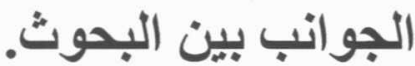
وتتطلق الاطروحه لتناقش موضوع هام يختص بالتقديرات الموزعه التقريبيه

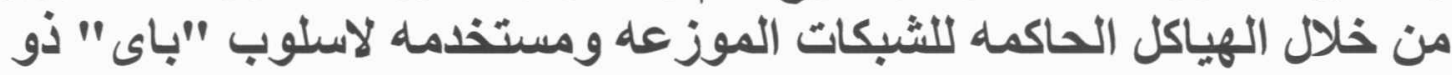

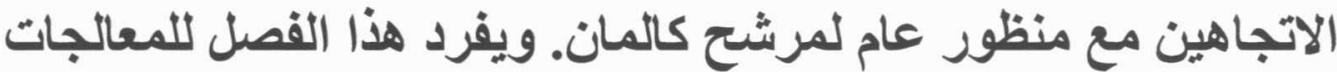

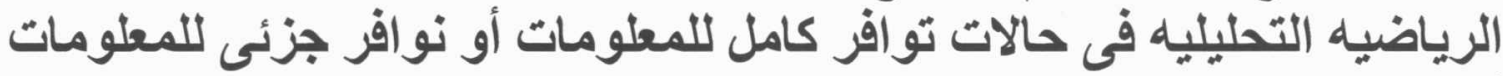

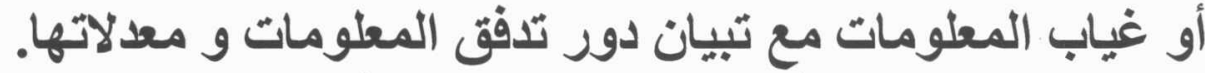

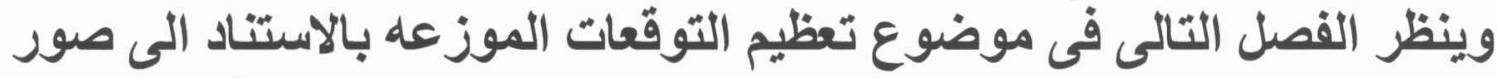
مصغره ل"منعم كالمان المتصل المفرد" ويقدم الفصل منعمات مثاليه مصغره

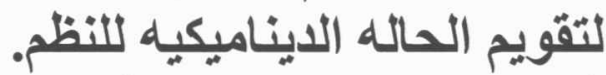

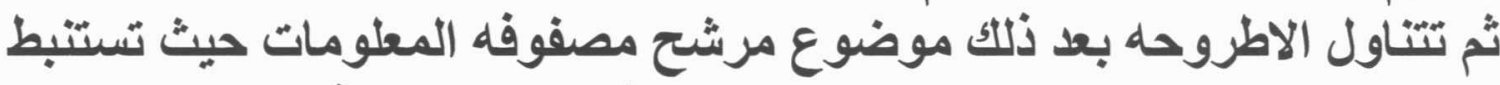

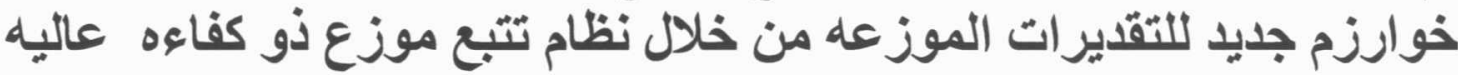

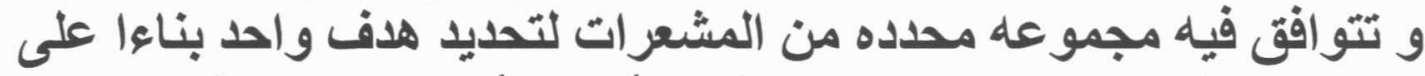

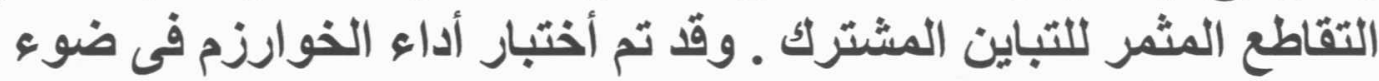

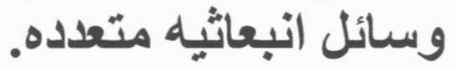

وقد أفردت الاطروحه فصول خاصه للمحاكاه على نماذج لعمليات عمليه و

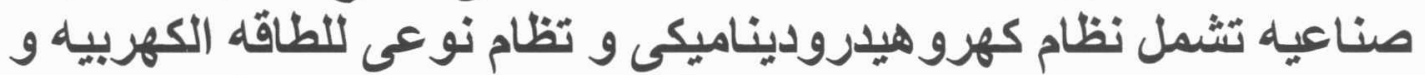
مر اجل صناعيه ـ وبينت النتائج مدى فعاليه النظم المستنبطه في في الاطروحيه. 


\section{NOMENCLATURE}

$\begin{array}{ll}\text { ABBREVIATIONS } & \\ \text { DKF } & : \text { Distributed Kalman Filtering } \\ \text { EM } & : \text { Expectation Maximization } \\ \text { KLPF } & : \text { Kalman-like Particle Filter } \\ \text { OOSM } & : \text { Out-of-Sequence Measurements } \\ \text { MSDF } & : \text { Multi-Sensor Data Fusion } \\ \text { DN } & : \text { Distributed Networks } \\ \text { DC } & : \text { Distributed Particle Filtering } \\ \text { DPF } & : \text { Self Tuning } \\ \text { ST } & : \text { Extended Kalman filtering } \\ \text { EKF } & : \text { Unscented Kalman filtering } \\ \text { UKF } & \end{array}$




\section{INTRODUCTION}

\subsection{Distributed Kalman Filtering}

In recent years, a compelling need has arisen to understand the effects of distributed information structures on estimation and filtering. Technological advances in hardware and software over the past few decades have enabled cheap and small, yet powerful, communication and computation devices leading to this field. The distributed system architecture, on the whole, is very powerful since it allows the design of the individual units or components to be much simpler, while not compromising too much on the performance. Additional benefits include increased robustness to component loss, increased flexibility in that the components can be reconfigured for many different tasks and so on. However, the design of such systems challenges various problems of assumptions, handling, fusing the architecture of such systems.

Distributed Kalman Filtering (DKF) in general shows scheme or class of schemes which employs Kalman filter either interconnected or spatially distributed. If the system by definition, employs sensor network, can process to employ Kalman filter, advancements (mass produced), in order to develop for multi-sensor network, multi-sensor data 
fusion, for this Kalman filter is an old scheme, and we need revised version of Kalman filters. Therefore in some cases, the conditions of standard Kalman filtering are violated and the regular recursive formulation can not be derived directly from the Kalman filtering theory and we have to propose methods for uncertain observations, passive packet loss, finite-time correlated noises etc.

Many advanced systems now make use of large number of sensors in practical applications ranging from aerospace and defense, robotics and automation systems, to the monitoring and control of a process generation plants. For example, an important practical problem in the above systems is to find an optimal state estimator given the observations. Moreover, DKF using applications of sensor fusion filter, federated square root filter, network of wireless cameras, multi-user detection problems, formation flying satellites, sparse large-scale systems, estimation on quantized observations etc. gives the route to DKF with applications.

The idea of distributing the computations involved in estimation problems using Kalman filters in sensor networks has been a subject of research since the late 1970s [1]. This section presents some of the recent contributions in this area.

OlfatiSaber [2] presented a distributed Kalman filter wherein a system with an dimensional measurement vector is first split into subsystems of -dimensional measurement vectors, then these subsystems are individually processed by micro Kalman filters in the nodes of the network. In this system, the sensors compute an average inverse covariance and average measurements using consensus filters. These averaged values are then used by each node to individually compute the estimated state of the 
system using the information form of the Kalman filter. Even though this approach is effective in an environment monitoring application where the state vector is partially known by each node in the network, it is not valid for an object tracking application where, at a given time, each node in a small number of nodes knows the entire state vector (although possibly not accurately).

Nettleton et al. [3] proposed a tree-based architecture in which each node computes the update equations of the Kalman filter in its information form and sends the results to its immediate predecessor in the tree. The predecessor then aggregates the received data and computes a new update. Node asynchrony is handled by predicting asynchronously received information to the current time in the receiving node. This approach is scalable since the information transmitted between any pair of nodes is fixed. However, the size of the information matrix is proportional to, where is the dimension of the state vector. In a sensor network setting, this information may be too large to be transmitted between nodes; therefore, methods to effectively quantize this information may need to be devised.

Regarding quantization, the work by Ribeiro et al. [4], studied a network environment wherein each node transmits a single bit per observation, the sign of innovation (SOI), at every iteration of the filter. The system assumes an underlying sensorscheduling mechanism so that only one node transmits the information at a time. It also assumes the update information (i.e., the signs of innovations) to be available to each node of the network. They showed that the mean squared error of their SOI Kalman filter is closely related to the error of a clairvoyant Kalman filter, which has access to 
all of the data in analog form. There is an interesting tradeoff between the works by Nettleton et al. and Ribeiro et al. The former presents a high level of locality (i.e., each node only needs information about its immediate neighbors). On the other hand, a reasonably large amount of information must be transmitted by each node. The later, by its turn, requires the transmission of a very small amount of information by each node; however, the algorithm does not present locality since the information must be propagated throughout the network. This kind of tradeoff must be carefully considered when designing an algorithm for real wireless sensor network applications.

To the best of our knowledge, the only work that applies Kalman filtering to a cluster-based architecture for object tracking using camera networks is that proposed by Goshorn et al. [5]. Their system assumes that the network is previously partitioned into clusters of cameras with similar fields of view. As the target moves, information within a cluster is handed off to a neighboring cluster.

\subsection{Research ObJectives and Methodology}

From the filtering and estimation perspective that we propose in this dissertation, the following is the main reason why the distributed filtering and estimation fusion problem is difficult.

\subsubsection{PROBLEM STATEMENT}

In distributed estimation problems, parallelism arises naturally due to the data obtained from different local sensors or subsystems located at various dispersed locations. Un- 
fortunately, due to limited communication bandwidth, or to increase survivability of the system in a poor environment, such as a war situation or in mission critical systems, every local sensor has to carry out filtering upon its own observations first for local requirement, and then transmit the processed data local state estimate to a fusion center. Therefore, the fusion center now needs to fuse all received local estimates to yield a globally optimal state estimate. Moreover, when the fusion takes place, the filtering job gets more challenging.

\subsection{Dissertation Structure}

The following is the dissertation structure for the chapters to follow. In chapter 2, we deal with the bibliographic literature survey of the distributed Kalman filtering, followed by chapter 3, which has the approximate distributed estimation of distributed Kalman filtering, followed by chapter 4, which has distributed EM-Based Kalman smoother. Chapter 5 contains the distributed estimation via information matrix approach. In the end is chapter 6 where conclusions and future perspectives are made.

\subsection{CONTRIBUTION}

The following are the research originalities and contributions of this dissertation.

- A comprehensive bibliographic review has been made where distributed Kalman filtering has been divided into eight classification.

- Bayesian-Based Forward Backward Kalman Filter has been derived, followed 
by three cases of Prior Information derived for Bayesian-Based Forward Backward Kalman Filter. Then two Techniques of Upper Bound and Lower Bound have been applied on three cases of Prior Information. In the simulation, various comparison simulations for electro-hydraulic system with faults have been made. In the end, time computation comparison of different techniques applied has been shown.

- Kalman-like particle smoother has been derived, followed by derivation and implementation of full-order Kalman-like particle smoother with EM algorithm, then the derivation and implementation of reduced-order Kalman-like particle smoother with EM algorithm has been made. In the simulation, power quality system simulation with comparison for full-order system and reduced-order system respectively have been made.

- Derivations and implementations have been made for the covariance intersectionbased information matrix filter, weighted covariance-based information matrix filter and Kalman-like particle filter-based information matrix filter respectively. In the simulation, industrial utility boiler simulation with comparison for various feedback strategies and measurement fusion methods has been made. 


\section{BIBLIOGRAPHIC REVIEW}

\subsection{AN OVERVIEW}

This chapter presents a bibliographic literature survey and technical review on Distributed Kalman Filtering.

\subsection{INTRODUCTION}

In hi-tech environment, a strict surveillance unit is required for an appropriate supervision. It often utilizes a group of distributed sensors which provide information of the local targets. Comparing with the centralized Kalman filtering (CKF), which can be used in mission critical scenarios, where every local sensor is important with its local information, the distributed fusion architecture has many advantages. There is no second thought that in certain scenarios, centralized Kalman filter plays a major role, and it involves minimum information loss. A general structure for the DKF can be seen in figure (see Fig. 2.1).

The distributed system architecture, on the whole, is very powerful since it allows the design of the individual units or components to be much simpler, while not compro- 


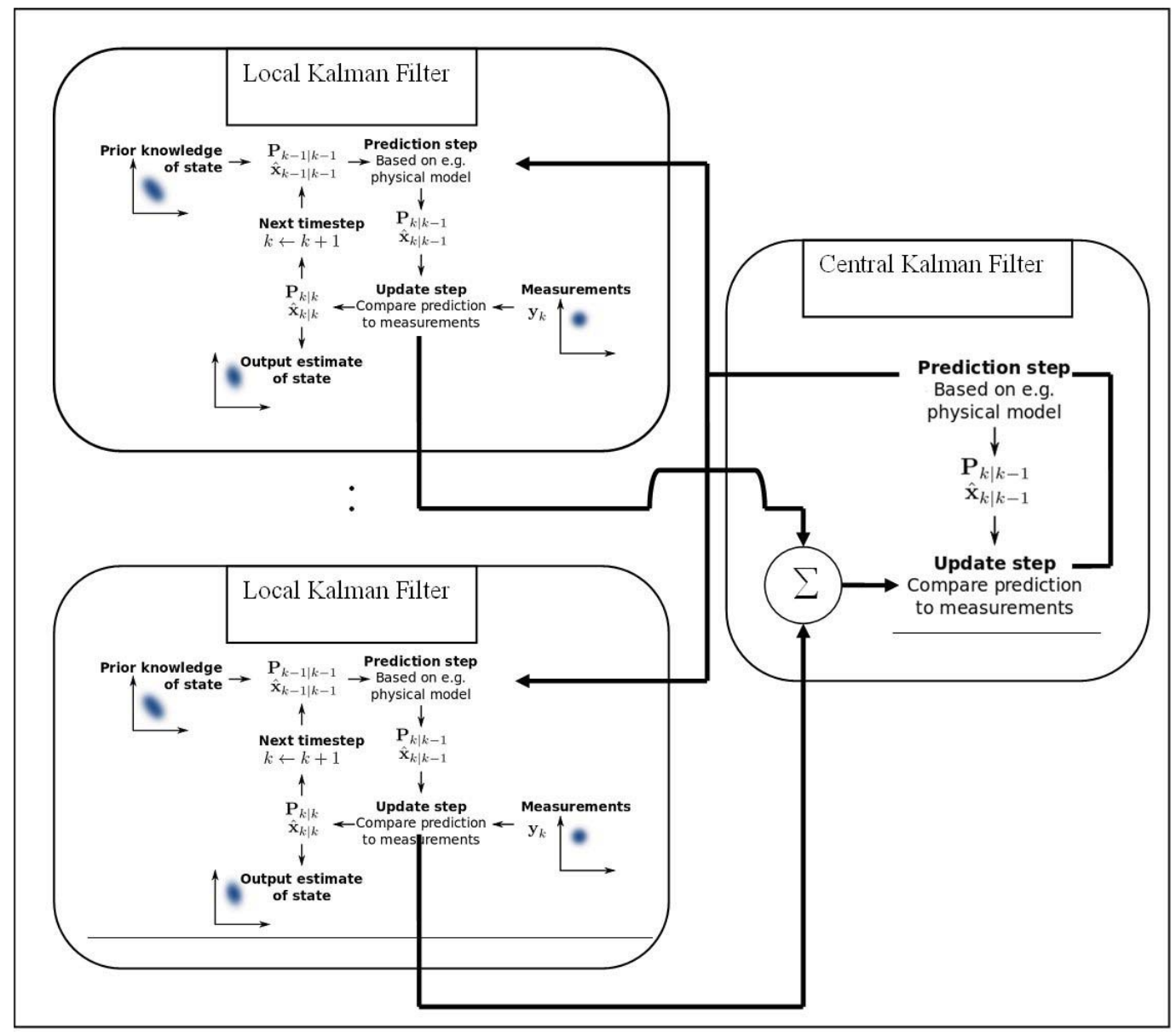

Figure 2.1: A general structure of DKF 
mising too much on the performance. Additional benefits include increased robustness to component loss, increased flexibility in that the components can be reconfigured for many different tasks and so on. However, the design of such systems challenges various problems of assumptions, handling, fusing the architecture of such systems. Our purpose is to provide a bibliographic survey on DKF and its architectures, comprising of distribution, fusion, filtering and estimation. A classification of such an architecture can be seen in the figure (see Fig. 2.2), which shows the vision of filtering and estimation under the umbrella of DKF. DKF methods have been categorized into eight main divisions which are then further categorized into other several subdivisions.

Therefore, in this paper, we present a bibliographic literature survey and technical review of DKF. The remaining part of the paper is organized as follows: Bibliographic review and technical survey of DKF and its applications are presented in Section II, diffusion-based DKF in Section III, followed by Distributed OOSM in Section IV, MSDF systems in section V, followed by DN in section VI, mathematical design in track-to-track fusion in Section VII, DC-based estimation in Section VIII, DPF in Section IX, ST-based distributed fusion Kalman filter in Section X. Finally some concluding remarks are given in Section XI. It should be noted that remark has been generated at the end of every section, showing the generic formulation generation explanation of a particular approach in that specific section.

$3 *$ The Fig. 2 is showing the classification of distributed Kalman filter, where KF stands for Kalman filter, DKF stands for distributed Kalman filter, EKF stands for extended Kalman filter, DC stands for distributed consensus, MSDF stands for multi-sensor data fusion, OOSM stands for out-of-sequence measurements, SN stands for sensor network, ST stands for self tuning, DPF stands for Distributed particle filter, DN stands for distributed networks. 


\begin{tabular}{|c|c|c|c|}
\hline $\mathrm{KF}$ & $\begin{array}{c}\text { DKF with } \\
\text { Applications }\end{array}$ & 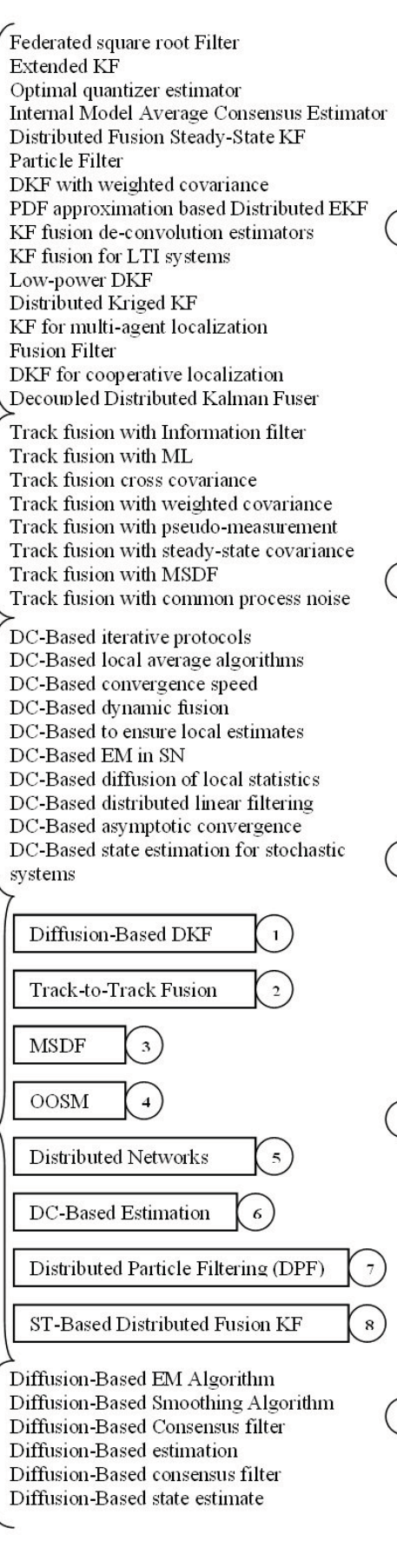 & $\begin{array}{l}\text { MSDF smoothing with ARMA signals } \\
\text { MSDF by scalars } \\
\text { MSDF centralized filter } \\
\text { MSDF with model uncertainty } \\
\text { MSDF with asynchronous measurements } \\
\text { MSDF with unified fusion models } \\
\text { MSDF with Bayesian adaptive framework } \\
\text { MSDF with alternates to quantile quantizer } \\
\text { MSDF with diagonal weighting matrix } \\
\text { MSDF with median fusion and information fusion } \\
\text { MSDF with BLUE fusion w/o prior information } \\
\text { MSDF with MAP } \\
\text { MSDF with one-step prediction } \\
\text { MSDF with weighted algorithms, uncertain } \\
\text { observations and correlated noises } \\
\text { MSDF with recursive fusion estimator } \\
\text { Multi-sensor EKF/de-convolution estimator } \\
\text { MSDF optimal linear fusion } \\
\text { OOSM with recursive BLUE } \\
\text { OOSM with cases of prior info. } \\
\text { OOSM with augmented state smoothing } \\
\text { OOSM with multi-step update } \\
\text { Multi-sensor OOSM problem } \\
\text { OOSM with one-step suboptimal updating } \\
\text { OOSM with optimal flight path update } \\
\text { Asynchronous OOSM } \\
\text { OOSM with estimation fusion } \\
\text { OOSM for linear systems } \\
\text { OOSM with more applications } \\
\text { DPF continuous transformation } \\
\text { DPF with delayed OOSM } \\
\text { DPF with MCMC smoothing step } \\
\text { DPF-Based on retrodiction } \\
\text { DPF with SOI estimation } \\
\text { DPF with tracking targets } \\
\text { DPF with tracking ground targets } \\
\text { DPF with individual sensor nodes } \\
\text { DPF and DKF Comparites } \\
\text { DN with Distributed EM consensus filter diffusion } \\
\text { DN with density estimation } \\
\text { DN with multi-sensor data } \\
\text { DN with spatially scattered sensors } \\
\text { DN with adaptive KF } \\
\text { DN with peer-to-peer architecture of DKF } \\
\text { DN with lossy network communicating multiple } \\
\text { DOAstion oOSM }\end{array}$ \\
\hline
\end{tabular}

Figure 2.2: Classification of Distributed Kalman Filter* 


\subsection{DKF Methods AND THEIR AppliCATIONS}

\subsubsection{DKF METHODS}

DKF can be introduced through different methods promoting to a better filtering approach, also considering various scenarios. A list of publications focusing on DKF methods and their applications is summarized in Table 2.1 and Table 2.2. In Table 2.1, the most recent references are [335] and [31], where in [335], a method is discussed under uncertain observations, including measurement with a false alarm probability as a special case. Moreover, it is proved that under a mild condition the fused state estimate is equivalent to the centralized Kalman filtering. In [31], consensus strategies of DKF are discussed where the problem of estimating the state of a dynamical system from distributed noisy measurements is considered with the help of a two-stage strategy for estimation. Other DKF methods and their applications can be seen in [7], [8], [9], [10], [101], [151], [152], [158], [162], [202], [203], [204], [205], [206], [276], [297], [298] and [300].

In Table 2.2, the most recent references are [25] and [33], where in [25], the estimation of sparsely connected, large scale systems is reported, moreover full distribution of Kalman filter is achieved. In [33], a network is modeled as a Bernoulli random topology and establish necessary and sufficient conditions for mean square sense and almost sure convergence of average consensus when network links fail. Other DKF methods and its applications can be seen in [26], [27], [28], [29], [30], [31], [32], [123], [153], [218], [219] and [220]. 
Remark 2.3.1 In [162], an $\ell$-sensor distributed dynamic system is described by:

$$
\begin{aligned}
x_{k+1} & =\phi_{k} x_{k}+v_{k}, k=0,1, \ldots \\
y_{k}^{i} & =H_{k}^{i} x_{k}+w_{k}^{i}, i=1, \ldots, \ell
\end{aligned}
$$

where $\phi_{k}$ is a matrix of order $r \times r, x_{k}, v_{k} \in \mathcal{R}^{r}, H_{i}^{k} \in \mathcal{R}^{N_{i} \times r}, y_{k}^{i}, w_{i}^{k} \in \mathcal{R}^{N_{i}}$. The process noise $v_{k}$ and measurement noise $w_{i}^{k}$ are both zero-mean random variables independent of each other temporally but $w_{i}^{k}$ and $w_{j}^{k}$ may be cross-correlated for $i \neq j$ at the same time instant $k$.

To compare performances between the centralized and distributed filtering fusion, the stacked measurement equation is written as:

$$
y_{k}=H_{k} x_{k}+w_{k}
$$

where

$$
\begin{aligned}
& y_{k}=\left(y_{k}^{1^{t}}, \ldots \ldots, y_{k}^{\ell^{t}}\right)^{t}, H_{k}=\left(H_{k}^{1^{t}}, \ldots \ldots, H_{k}^{\ell^{t}}\right)^{t} \\
& w_{k}=\left(w_{k}^{1^{t}}, \ldots \ldots, w_{k}^{\ell^{t}}\right)^{t}
\end{aligned}
$$

and the covariance of the noise $w_{k}$ is given by:

$$
\operatorname{Cov}\left(w_{k}\right)=R_{k}, R_{k}^{i}=\operatorname{Cov}\left(w_{k}^{i}\right), \quad i=1, \ldots, \ell
$$

where $R_{k}$ and $R_{k}^{i}$ are both invertible for all $i$. According to the standard results of 
Kalman filtering, the local Kalman filtering at the $i$-th sensor is expressed as:

$$
\begin{aligned}
& \widehat{K}_{k}^{i}=\widehat{P}_{k / k}^{i} H_{k}^{i^{t}} \widehat{R}_{k}^{i^{-1}} \\
& \widehat{x}_{k / k}^{i}=\widehat{x}_{k / k-1}^{i}+\widehat{K}_{k}^{i}\left(y_{k}^{i}-H_{k}^{i} \widehat{x}_{k / k-1}^{i}\right) \\
& \widehat{P}_{k / k}^{i}=\widehat{P}_{k / k-1}^{i}-\widehat{K}_{k}^{i} H_{k} \widehat{P}_{k / k-1}^{i}
\end{aligned}
$$

where, the covariance of filtering error can be stated as:

$$
\widehat{P}_{k / k}^{i-1}=\widehat{P}_{k / k-1}^{i-1}+H_{k}^{i t} \widehat{R}_{k}^{i^{-1}} H_{k}^{i}
$$

with

$$
\begin{aligned}
\widehat{x}_{k / k-1}^{i} & =\widehat{\Phi}_{k} \widehat{x}_{k-1 / k-1}^{i}, \\
\widehat{P}_{k / k}^{i} & =E\left[\left(\widehat{x}_{k / k}^{i}-\widehat{x}_{k}\right)\left(\widehat{x}_{k / k-1}^{i}-\widehat{x}_{k}\right)^{t}\right] \\
\widehat{P}_{k / k-1}^{i} & =E\left[\left(\widehat{x}_{k / k-1}^{i}-\widehat{x}_{k}\right)\left(\widehat{x}_{k / k-1}^{i}-\widehat{x}_{k}\right)^{t}\right]
\end{aligned}
$$

Similarly, the centralized Kalman filtering with all sensor data is given by:

$$
\begin{aligned}
& \widehat{K}_{k}=\widehat{P}_{k / k} H_{k}^{t} \widehat{R}_{k}^{-1} \\
& \widehat{x}_{k / k}=\widehat{x}_{k / k-1}+\widehat{K}_{k}\left(y_{k}-H_{k} \widehat{x}_{k / k-1}\right) \\
& \widehat{P}_{k / k}=\widehat{P}_{k / k-1}-\widehat{K}_{k} H_{k} \widehat{P}_{k / k-1}
\end{aligned}
$$


where, the covariance of filtering error can be described as:

$$
\widehat{P}_{k / k}^{-1}=\widehat{P}_{k / k-1}^{-1}+H_{k}{ }^{t} \widehat{R}_{k}^{-1} H_{k}
$$

with

$$
\begin{aligned}
\widehat{x}_{k / k-1} & =\widehat{\Phi}_{k} \widehat{x}_{k-1 / k-1} \\
\widehat{P}_{k / k} & =E\left[\left(\widehat{x}_{k / k}-\widehat{x}_{k}\right)\left(\widehat{x}_{k / k-1}-\widehat{x}_{k}\right)^{t}\right] \\
\widehat{P}_{k / k-1} & =E\left[\left(\widehat{x}_{k / k-1}-\widehat{x}_{k}\right)\left(\widehat{x}_{k / k-1}-\widehat{x}_{k}\right)^{t}\right]
\end{aligned}
$$

It is quite clear when the sensor noises are cross-dependent that

$$
H_{k}^{t} \widehat{R}_{k}^{-1} H_{k}=\sum_{i=1}^{l} H_{k}^{i^{t}} \widehat{R}_{k}^{i^{-1}} H_{k}^{i}
$$

Likewise, the centralized filtering and error matrix could be explicitly expressed in terms of the local filtering and error matrices as follows:

$$
\widehat{P}_{k / k}^{-1}=\widehat{P}_{k / k-1}^{-1}+\sum_{i=1}^{l}\left(\widehat{P}_{k / k}^{i^{-1}}-\widehat{P}_{k / k-1}^{i^{-1}}\right)
$$

and

$$
\widehat{P}_{k / k}^{-1} \widehat{x}_{k / k}=\widehat{P}_{k / k-1}^{-1}+\sum_{i=1}^{l}\left(\widehat{P}_{k / k}^{i-1} \widehat{x}_{k / k}^{i}-\widehat{P}_{k / k-1}^{i^{-1}} \widehat{x}_{k / k-1}^{i}\right)
$$


Also,

$$
H_{k}^{i^{\prime}} \widehat{R}_{k}^{i^{-1}} y_{k}^{i}=\widehat{P}_{k / k}^{i-1} \widehat{x}_{k / k}^{i}-\widehat{P}_{k / k-1}^{i-1} \widehat{x}_{k / k-1}^{i}
$$

Proposition 2.1 In what follows is the detailed bibliographic review of DKF methods which have been explained comprehensively in Table 2.1 and Table 2.2 respectively. The recent references have been explained and others have been cited in the tables. In the end [162] considering the distributed dynamic systems for DKF has been explained as a particular case.

\subsubsection{DKF WITH APPLICATIONS}

This section shows the characterization of DKF with various applications. A list of publications in some application-oriented research is summarized in Table 2.3 and Table 2.4 respectively. As it can be seen, a large amount of research has been carried out in the framework of modified filters. In Table 2.3, the most recent ones are as follows. In [186], the synthesis of a distributed algorithm is made to compute weighted least squares estimates with sensor measurements correlated. In [199], distributed object tracking system which employs a cluster-based Kalman filter in a network of wireless cameras is presented. In [211] [212], distributed recursive mean-square error optimal quantizer-estimator based on the quantized observations is presented. Other DKF applications can be seen in [335], [336], [338], [339] .[38], [39], [40], [41], [42], [43], [44], [105], [106], [109], [114], [119], [156], [179], [191], [197], [213], [214], [215], [216], [221], [233], [237], [238] and [242]. 
Table 2.1: DKF Methods I

\begin{tabular}{l}
\hline \hline DKF Design Approaches Used \\
\hline - Under uncertain observations, including measurement
\end{tabular}

with a false alarm probability

- Under uncertain observations, randomly variant dynamic

systems with multiple models

- Optimal centralized and distributed fusers are

algebraically equivalent in this case

- Power systems: mode estimation. A trust-based DKF

approach to estimate the modes of power systems

- Using Standard Kalman filter locally, together with a consensus

step in order to ensure that the local estimates agree

- Frequency-domain characterization of the distributed estimator's

steady-state performance

- EKF to globally optimal KF for the dynamic systems with finite-time

correlated noises

- Distributed Kalman-type processing scheme essentially makes use of the

fact that the sensor measurements do not enter into the update equation for the estimation error covariance matrices

- DKF fusion with weighted covariance approach

- DKF fusion with passive packet loss or initiative intermittent

communications from local estimators to a fusion center while the process noise does exist

- For each Kalman update, an infinite number of consensus steps

to restricted to one

- For each Kalman update, state estimates are additionally exchanged

- Only the estimates at each Kalman update over-head are exchanged

- Analyzes the number of messages to exchange between successive updates in DKF

- Global Optimality of DKF fusion exactly equal to the corresponding centralized optimal Kalman filtering fusion

- A parallel and distributed state estimation structure developed

from an hierarchical estimation structure

- A computational procedure to transform an hierarchical Kalman filter

into a partially decentralized estimation structure

- Optimal DKF based on a-priori determination of measurements

[202] [203]

[204] 
Table 2.2: DKF Methods II

\begin{tabular}{l|r}
\hline \hline DKF & References \\
\hline - Estimate sparsely connected, large scale systems & {$[25]$} \\
- $n$-th order with multiple sensors & {$[26]$} \\
- Data-fusion over arbitrary communication networks & {$[27]$} \\
- Iterative consensus protocols & {$[28]$} \\
- Using bipartite fusion graphs & {$[29]$} \\
- Local average consensus algorithms & {$[30]$} \\
- Based on consensus strategies & {$[31]$} \\
- Semi-definite programming -based consensus Iterations & {$[32]$} \\
- Converge Speed of consensus strategies & {$[33]$} \\
- Distributed Kalman filtering, with focus on limiting the & \\
required communication bandwidth & {$[123]$} \\
- Distributed Kalman-type processing scheme, which provides & \\
optimal track-to-track fusion results at arbitrarily chosen & \\
instants of time & {$[153]$} \\
- Distributed architecture of track-to-track & \\
fusion for computing the fused estimate from multiple filters & \\
tracking a maneuvering target with the simplified maximum & \\
likelihood estimator & {$[218]$} \\
- Original batch form of the Maximum Likelihood (ML) estimator & {$[219]$} \\
- Modified Probabilistic Neural Network & {$[220]$} \\
\hline
\end{tabular}


In Table 2.4, the most recent one are as follows. Low-power DKF based on a fast polynomial filter is shown in [267]. Distributed 'Kriged' Kalman filtering is addressed in [272]. Decoupled distributed Kalman fuser presented by using Kalman filtering method and white noise estimation theory is shown in [281]. Decomposition of a linear process model into a cascade of simpler subsystems is given in [282]. Other applications can be seen in [7], [338], [247], [248], [268], [269], [270], [271], [273], [275], [283], [284], [299], [321], [323], and [324] respectively.

Proposition 2.2 In what follows is the detailed bibliographic review of DKF methods with applications which have been explained comprehensively in Table 2.3 and Table 2.4 respectively. The recent references have been explained and others have been cited in the tables.

\subsection{DIFFUSION-BASED DKF}

The publications of diffusion-based DKF are classified in Table 2.5. Recent ones in this area are as follows. Diffusion-based distributed expected maximization (EM) algorithm for Gaussian mixtures is shown in [50]. Diffusion-based Kalman filtering and smoothing algorithm is shown in [51]. Diffusion Kalman filtering for every measurement and for every node, a local state estimate using the data from the neighborhood is provided in [178]. Other publications classified with diffusion-based DKF are [97], [99], [173], [174], [175], [176] and [177] respectively.

Remark 2.4.1 In the paper [50], a diffusion scheme of EM(DEM) algorithm for Gaussian mixtures in Wireless Sensor Networks (WSNs) is proposed. At each iteration, the 
Table 2.3: DKF with Applications I

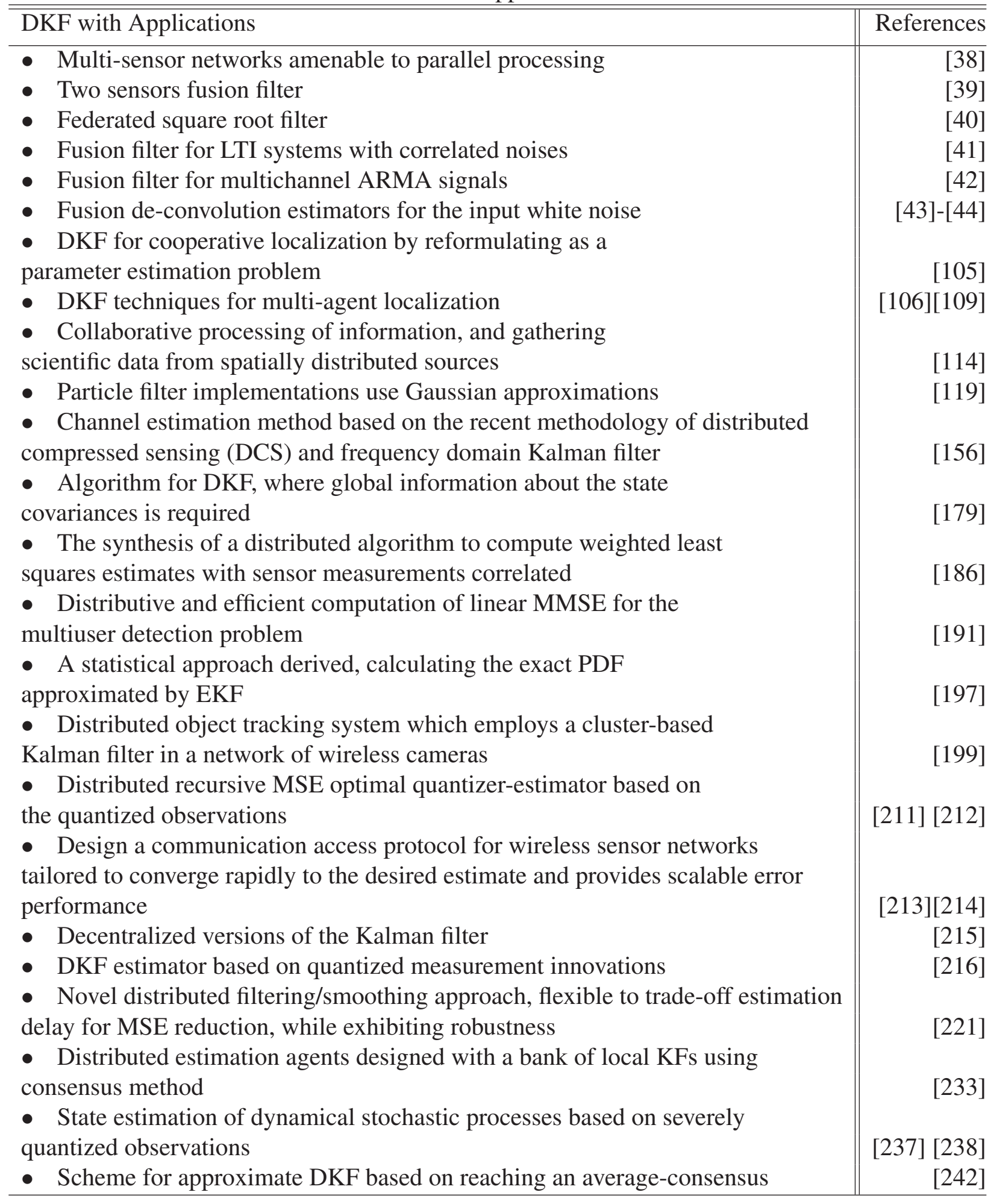


Table 2.4: DKF with Applications II

\begin{tabular}{l||r}
\hline \hline DKF with Applications & References \\
\hline - When no feedback from the fusion center to local sensors, a distributed Kalman & \\
filtering fusion formula under a mild condition & {$[247]$} \\
- Rigorous performance analysis for KF fusion with feedback & {$[248]$} \\
- Low-power DKF based on a fast polynomial filter & {$[267]$} \\
- Consensus Problem and their special cases & {$[268]$} \\
- DKF for sparse large-scale systems monitored by sensor networks & {$[269]$} \\
- DKF to estimate actuator faults for deep space formation flying satellites & {$[270]$} \\
- Internal model average consensus estimator for DKF & {$[271]$} \\
- Distributed Kriged Kalman filtering & {$[272]$} \\
- The behavior of the distributed Kalman filter varies smoothly from a & \\
centralized Kalman filter to a local Kalman filter with average consensus update & {$[273]$} \\
- Track fusion formulas with feedback are, like the track fusion without feedback & {$[275]$} \\
- Decoupled distributed Kalman fuser presented by using Kalman filtering & \\
method and white noise estimation theory & {$[281]$} \\
- Decomposition of a linear process model into a cascade of simpler subsystems & {$[282]$} \\
- Distributed fusion steady-state Kalman filtering by using the modern time & \\
series analysis method & {$[283]$} \\
- Distributed Kalman filtering with weighted covariance transfer function & {$[284]$} \\
describing the error behavior of the DKF in the case of stationary noise processes & {$[299]$} \\
- DKF approach for distributed parametric systems, for deep space & {$[321][323]$} \\
formations, for unreliable information, for false alarms respectively & {$[324][326]$} \\
\hline
\end{tabular}


time-varying communication network is modeled as a random graph. A diffusion-step (D-step) is implemented between the E-step and the M-step. In the E-step, sensor nodes compute the local statistics by using local observation data and parameters estimated at the last iteration. In the D-step, each node exchanges local information only with its current neighbors and updates the local statistics with exchanged information. In the $M$-step, the sensor nodes compute the estimation of parameter using the updated local statistics by the D-step at this iteration. Compared with the existing distributed EM algorithms, the proposed approach can extensively save communication for each sensor node while maintain the estimation performance. Different from the linear estimation methods such as the least-squares and the least-mean squares estimation algorithms, each iteration of EM algorithm is a nonlinear transform of measurements. The steadystate performance of the proposed DEM algorithm can not be analyzed by linear way. Instead, we show that the DEM algorithm can be considered as a stochastic approximation method to find the maximum likelihood estimation for Gaussian Mixtures. In this regard, we have in mind a network of $M$ sensor nodes is considered, each of which has $N_{m}$ data observations $\left\{y_{m, n}\right\}, m=1,2, \ldots ., M, n=1,2, \ldots . ., N_{m}$. These observations are drawn from a $K$ Gaussian mixtures with mixture probabilities $\alpha_{1}, \ldots ., \alpha_{k}$.

$$
y_{m, n} \sim \sum_{j=1}^{K} \alpha_{j} . N\left(\mu_{j}, \Sigma_{j}\right)
$$

where $N(\mu, \Sigma)$ denote the Gaussian density function with mean $\mu$ and covariance $\Sigma$. Let $z \in\{1,2, \ldots, K\}$ denote the missing data where Gaussian y comes from.

Proposition 2.3 In what follows is the detailed bibliographic review of diffusion-based 
Table 2.5: Diffusion-Based DKF

\begin{tabular}{|c|c|}
\hline$\overline{\text { Diffusion App }}$ & Refe \\
\hline $\begin{array}{l}\text { - Diffusion-Based Distributed EM algorithm for Gaussian } \\
\text { mixtures } \\
\text { - Diffusion-Based Kalman filtering and smoothing algorithm } \\
\text { - Distributed EM algorithm over sensor networks, consensus } \\
\text { filter used to diffuse local sufficient statistics to neighbors } \\
\text { and estimate global sufficient statistics in each node } \\
\text { - Consensus filter diffusion of local sufficient statistics } \\
\text { over the entire network through communication with } \\
\text { neighbor nodes } \\
\text { - Diffusion Kalman filtering, where nodes communicate } \\
\text { only with their neighbors, and no fusion center is present } \\
\text { - DKF proposed in the context of diffusion estimation } \\
\text { - DKF proposed in the context of average consensus } \\
\text { - Diffusion Kalman filtering for every measurement } \\
\text { and for every node, a local state estimate using the } \\
\text { data from the neighborhood }\end{array}$ & $\begin{array}{l}{[50]} \\
{[51]}\end{array}$ \\
\hline
\end{tabular}

DKF methods which have been explained comprehensively in Table 2.5. The recent references have been explained and others have been cited in the Table. In the end [50] considering the diffusion scheme for Gaussian mixture in wireless sensor network has been explained as a particular case.

\subsection{DistRibUTED OOSM}

This section shows the discussion on distributed OOS. Typically OOSM behavior is caused by deterministic transmission system, where the transmission time of a message vary very much. Distributed OOSM-based list of publications are classified in Table 2.6. The most recent publications in distributed OOSM are [138]-[143], [164], [194] and [279], where efficient incorporation of OOSMs in Kalman filters is developed in [138]-[143]. Counterpart of the OOSM update problem, needed to remove an 
earlier measurement from the flight path, is analyzed in [164]. Focus on centralized update problem for multiple local sensor systems with asynchronous OOSMs is treated in [194]. A globally optimal state trajectory update algorithm for a sequence with arbitrary delayed OOSMs including the case of interlaced OOSMs with less storages is given in [279]. Other publications classified with distributed OOSM are [61], [62], [63], [64], [81], [82], [83], [84], [85], [86], [87], [88], [89], [90], [141], [163], [165], [166], [167], [168], [188], [195], [207], [208], [224], [225], [225]-[229], [230], [231], [280], [301] and [302].

Proposition 2.4 In what follows is the bibliographic review of OOSM, a subdivision of DKF which have been explained comprehensively in Table 2.6. The recent references have been explained and others have been cited in the Table.

\subsection{MSDF SYSTEMS}

This section shows the discussion on another division of DKF with respect to MSDF systems. In Tables 2.7, 2.8 and 2.9, MSDF systems-based list of publications are classified respectively. The most recent of the publications described in these tables are as follows. Sensor noises of converted system cross-correlated, and also correlated with the original system is treated in [335]. Centralized fusion center, expressed by a linear combination of the local estimates is presented in [336]. Bayesian framework for adaptive quantization, fusion-center feedback, and estimation of a spatial random field and its parameters are treated in [65]. A framework for alternates to quantile quantizer and fusion center is provided in [66]. Median fusion and information fusion, not based 
Table 2.6: OOSM

\begin{tabular}{l}
\hline \hline OOSM Approaches \\
- Recursive BLUE without prior \\
- Cases of prior information about the OOSM \\
- Dating the state estimate globally optimally \\
with three cases of prior information about OOSM
\end{tabular}

- Updating the state estimate globally optimally with an OOSM within one step time delay for a system

- Multi-step OOSM updating using augmented state smoothing

- Multi-step update in OOSM

- Multi-sensor OOSM problem in a cluttered environment

- One-step suboptimal updating algorithms with a nonsingular state transition matrix

- Efficient incorporation of OOSMs in KFs

- A globally optimal flight path update algorithm with OOSMs

- Counterpart of the OOSM update problem, needed to remove an earlier measurement from flight path

- One-step solution for the general OOSM problem in tracking presented independently

- Distributed fusion update for the local sensors with OOSMs

- OOSM with practical applications

- Optimal analysis of one-step OOSM filtering algorithms in target tracking

- Focus on centralized update problem for multiple local sensor systems with asynchronous OOSMs

- The $l$ step algorithm developed for OOSM

- Optimal distributed estimation fusion with OOSM at local sensors

- Two new algorithms for solving the out-of-sequence data problem for the case of linear and nonlinear dynamic control systems

- When the delays and the sequence of arrival of all the information are not fixed, constituting the named Out-Of-Sequence Problem (OOSP)

- Out-Of-Sequence Problem (OOSP) developed for linear systems

- OOSP developed for non-linear systems

- A globally optimal state trajectory update algorithm for a sequence with arbitrary delayed OOSMs including the case of interlaced OOSMs with less storages

- OOSM with more applications

- OOSM processing for tracking ground target using particle filters

- Comparison of the KF and particle filter based OOSM filtering algorithms 
on weighted sums of local estimates, are presented in [92]. Optimal distributed estimation fusion algorithm with the transformed data is proposed in [125]. Corresponding distributed fusion problem, proposed based on a unified data model for linear unbiased estimator is presented in [128]. An algorithm, fuses one step predictions at both the fusion center and all current sensor estimates is given in [129]. In multi-sensor linear dynamic system, several efficient algorithms of centralized sensor fusion, distributed sensor fusion, and multi-algorithm fusion to minimize the Euclidian estimation error of the state vector are documented in [130]. Problem of data fusion in a decentralized and distributed network of multi-sensor processing nodes is contained in [193]. Fusion algorithm based on multi-sensor systems and a distributed multi-sensor data fusion algorithm based on Kalman filtering is presented in [274]. Other related publications cited in the Table 2.7 are [338]-[339], [337], [332], [333, 334], [37], [46], [47], [48], [48], [60], [59, 58], [57], [56], [55], [54], [53], [52]. Other related publications cited in the Table 2.8 are [67], [68], [91], [93, 94], [95], [115], [116], [117], [124], [125], [127] and [128]. Other related publications cited in the Table 2.9 are [131], [169, 170], [170, 171, 172], [183], [187], [200], [240], [241], [244], [249], [252], [253], [254, 255, 256], [257], [259], [275], [277], [278], [304] and [307].

Remark 2.6.1 In [332], using estimators of white measurement noise, an optimal information fusion distributed Kalman smoother is given for multichannel ARMA signals with correlated noise. The work on ARMA signal and information fusion is also done in [333] and [334]. Basically it has a three-layer fusion structure with fault tolerant, and robust properties. The first fusion layer and the second fusion layer both have nested 
parallel structures to determine the prediction error cross-covariance of the state and the smoothing error cross-covariance of the ARMA signal between any two faultless sensors at each time step. And the third fusion layer is the fusion centre to determine the optimal matrix weights and obtain the optimal fusion distributed smoother for ARMA signals. The computation formula of smoothing error cross-covariance matrix between any two sensors is given for white measurement noise. The computation formula of smoothing error cross-covariance matrix between any two sensors is given for white measurement noise. The discrete time multi-channel ARMA signal system considered here with L sensors is:

$$
\begin{aligned}
B\left(q^{-1}\right) s(t) & =C\left(q^{-1}\right) w(t) \\
y_{i}(t) & =s(t)+v_{i}(t), i=1, \ldots, L
\end{aligned}
$$

where $s(t) \in \Re^{m}$ is the signal to estimate, $y_{i}(t) \in \Re^{m}$ is the measurement of the $i t h$ sensor, $w(t) \in \Re^{r}$ is the process noise, $v_{i}(t) \in \Re^{m}$ is the measurement noise of the ith sensor, $L$ is the number of sensors, and $B\left(q^{-1}\right), C\left(q^{-1}\right)$ are polynomial matrices having the form

$$
X\left(q^{-1}\right)=X_{0}+X_{1}\left(q^{-1}\right)+\ldots \ldots+X_{n_{x}} q^{-n_{x}}
$$

where the argument $q^{-1}$ is the back shift operator, that is, $q^{-1} x(t)=x(t-1), \quad X_{i}, i=$ $0,1, \ldots . ., n_{x}$ are the coefficient matrices, the degree of $X\left(q^{-1}\right)$ is denoted by $n_{x}$.

In the multi-sensor random parameter matrices case, sometimes, even if the origi- 
nal sensor noises are mutually independent, the sensor noises of the converted system are still cross-correlated. Hence, such multi-sensor system seems not satisfying the conditions for the distributed Kalman filtering fusion as given in [338, 339]. In the paper [335], it was proved that when the sensor noises or the random measurement matrices of the original system are correlated across sensors, the sensor noises of the converted system are cross-correlated. Even if so, similarly with [336], centralized random parameter matrices Kalman filtering, where the fusion center can receive all sensor measurements, can still be expressed by a linear combination of the local estimates. Therefore, the performance of the distributed filtering fusion is the same as that of the centralized fusion under the assumption that the expectations of all sensor measurement matrices are of full row rank. Numerical examples are given which support our analysis and show significant performance loss of ignoring the randomness of the parameter matrices. The following discrete time dynamic system is considered:

$$
\begin{aligned}
x_{k+1} & =F_{k} x_{k}+v_{k} \\
y_{k} & =H_{k} x_{k}+\omega_{k}, k=0,1,2,3, \ldots
\end{aligned}
$$

where $x_{k} \in \Re^{r}$ is the system state, $y_{k} \in \Re^{N}$ is the measurement matrix, $v_{k} \in \Re^{r}$ is the process noise, and $\omega_{k} \in \Re^{N}$ is the measurement noise. The subscript $k$ is the time index. $F_{k} \in \Re^{r \times r}$ and $H_{k} \in \Re^{N \times r}$ are random matrices.

Proposition 2.5 In what follows is the detailed bibliographic review of MSDF methods which have been explained comprehensively in Table 2.7, Table 2.8 and Table 2.9 re- 
Table 2.7: MSDF Systems I

\begin{tabular}{l}
\hline \hline MSDF Design Approaches \\
- Sensor noises of converted systems cross-correlated, whilst original \\
system independent
\end{tabular}

- Sensor noises of converted system cross-correlated, whilst original system also correlated

- Centralized fusion center, expressed by a linear combination of the local estimates

- No centralized fusion center, but algorithm highly resilient to lose one or more sensing nodes

- Discrete smoothing fusion with ARMA Signals LMV with information fusion filter

- Deconvolution estimation of ARMA signal with multiple sensors

- Fusion criterion weighted by scalars

- Functional equivalence of two measurement fusion methods

- Centralized filter, data processed/communicated centrally

- New performance bound for sensor fusion with model uncertainty

- All prior fusion results with Asynchronous Measurements

- Unified fusion model and unified batch fusion rules

- Unified rules by examples

- Computing formulation for cross-covariance of the local estimation

- Conditions for centralized and distributed fusers to be identical References

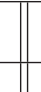

- Relationships among the various fusion rules

- Optimal rules for each sensor to compress its measurements

- Various issues unique to fusion for dynamic systems

- Bayesian framework for adaptive quantization, fusion-center feedback, and estimation of a spatial random field and its parameters

- Framework for alternates to quantile quantizer and fusion center 
Table 2.8: MSDF II

\begin{tabular}{|c|c|}
\hline MS & \\
\hline $\begin{array}{l}\text { - Diagonal weighting matrices } \\
\text { - Different fusion rates for the different states } \\
\text { - Optimal distributed estimation fusion in the LMV estimation } \\
\text { - Median fusion and information fusion, not based on weighted sums } \\
\text { of local estimates } \\
\text { - Distributed filtering algorithms, optimal in mean square sense linear } \\
\text { combinations of the matrix or scalar weights with derivations } \\
\text { - Closed form analytical solution of steady fused covariance } \\
\text { of information matrix fusion with arbitrary number of sensor derived } \\
\text { - Focus on various issues unique to fusion for dynamic systems, } \\
\text { present a general data model for discretized asynchronous } \\
\text { multi-sensor systems } \\
\text { - Recursive BLUE fusion without prior information } \\
\text { - Statistical interval estimation fusion } \\
\text { - Fused estimate communicated to a central node } \\
\text { to be used for some task } \\
\text { - Optimal distributed estimation fusion algorithm } \\
\text { with the transformed data is proposed, which is actually equivalent } \\
\text { to the centralized estimation fusion } \\
\text { - State estimation fusion algorithm, optimal in the sense of MAP } \\
\text { - Corresponding distributed fusion problem, proposed based } \\
\text { on a unified data model for linear unbiased estimator } \\
\text { - An algorithm, fuses one step predictions at both } \\
\text { the fusion center and all current sensor estimates } \\
\text { - In multi-sensor linear dynamic system, several efficient algorithms of } \\
\text { centralized sensor fusion, distributed sensor fusion, and multi-algorithm } \\
\text { fusion to minimize the Euclidian estimation error of the } \\
\text { state vector }\end{array}$ & $\begin{array}{l}{[115]} \\
{[116]}\end{array}$ \\
\hline
\end{tabular}


Table 2.9: MSDF III

\begin{tabular}{l}
\hline MSDF Design Approaches \\
- Derivation of approximation technique for arbitrary probability densities,
\end{tabular}
providing distributable fusion structure as the linear information filter - Multi-sensor distributed fusion filters based on three weighted algorithms, applied to the systems with uncertain observations and correlated noises

- MSDF in state estimation fields, and easy fault detection, isolation and more reliability

- CKF algorithm, obtained by combining all measurement data

- Design of general and optimal asynchronous recursive fusion estimator for a kind of multi-sensor asynchronous sampling system

- Problem of data fusion in a decentralized and DN of multi-sensor processing nodes

- To assure the validity of data fusion, a centralized trust rating system

- white noise filter weighted by scalars based on Kalman predictor

- White noise de-convolution estimators

- Optimal information fusion distributed Kalman smoother given for discrete time ARMA signals

- Optimal dimensionality reduction of sensor data by using the matrix decomposition, pseudo-inverse, and eigenvalue techniques

- Multi-sensor Information fusion distributed KF and applications

- Based on analysis of the fused state estimate covariances of the two measurement fusion methods

- MSDF approaches to resolve problem of obtaining a joint state-vector estimate

- Decentralized multi-sensor EKF which has been divided up into modules

- A distributed reduced-order fusion Kalman filter (DRFKF)

- Fusion algorithm based on multi-sensor systems and a distributed MSDF algorithm based on KF

- Track fusion formulas with feedback are, like the track fusion without feedback

- The optimal distributed KF fusion algorithms for the various cases

- General optimal linear fusion

- Information fusion in distributed SN

- Multi-scale Recursive Estimation, Data Fusion, and Regularization References 
spectively. The recent references have been explained and others have been cited in the Tables. In the end [332] considering the optimal information fusion distributed Kalman smoother has been explained as a particular case.

\subsection{DNs}

This section describes the area of DNs in DKF. The list of publications on DNs is classified in Table 2.10. Some recent publications in this area are as follows. Distributed expectation maximization (EM) algorithm over sensor networks, consensus filter used to diffuse local sufficient statistics to neighbors and estimate global sufficient statistics in each node are developed in [97]. Modified adaptive Kalman filter for sensor-less current control of a three-phase inverter based distributed generation system is proposed in [196]. Distributed estimation scheme for tracking the state of a Gauss-Markov model by means of observations at sensors connected in a network is the subject of [201]. A message-passing version of the Kalman consensus filter (KCF) is considered in [209]. For decentralized tracking applications, DKF and smoothing algorithms are derived for any-time MMSE optimal consensus-based state estimation using Wireless Sensor Networks are considered in [217]. Other publications cited in Table 2.10 are [69], [70], [98, 100], [99], [132], [154], [155], [192], [210], [223], [232], [234], [313]-[319] and [325].

Remark 2.7.1 In literature, a single plant is usually assumed for an NCS and the links between the plant and the estimator or controller channel. This notion is extended by a distributed networked control system (DNCS) in which there are multiple agents 
communicating over a lossy communication channel [69]. A DNCS extends an NCS to model a distributed multi-agent system such as the Vicsek model. The best examples of such system include ad-hoc wireless sensor networks and a network of mobile agents. The exact state estimation method based on the Kalman filter is introduced in [69]. However, the time complexity of the exact method can be exponential in the number of communication links.are closed by a common (unreliable) communication In the paper [70], this issue is addressed by developing two approximate filtering algorithms for estimating states of a DNCS. The approximate filtering algorithms bound the state estimation error of the exact filtering algorithm and the time complexity of approximate methods is not dependent on the number of communication links. The stability of estimators under a lossy communication channel is studied in [309], [310]. However, the extension of the result to the general case with an arbitrary number of lossy communication links is unknown. While computing the exact communication link probabilities required for stable state estimation is non-trivial, the general conditions for stable state estimation using jump linear system theory are described. The following first distributed control system consisting of $N$ agents is considered, in which there is no communication loss. The discrete-time linear dynamic model of the agent $j$ can be described as following:

$$
x_{j}(k+1)=\sum_{i=1}^{N} A_{i j} x_{i}(k)+G_{j} w_{j}(k)
$$

where $k \in \mathbf{Z}^{+}, x_{j}(k) \in \mathbf{R}^{n_{x}}$ is the state of the agent $j$ at time $k, w_{j}(k) \in \mathbf{R}^{n_{w}}$ is a white noise process, $A_{i j} \in \mathbf{R}^{n_{x} \times n_{x}}$, and $G_{j} \in \mathbf{R}^{n_{x} \times n_{W}}$. Hence, the state of the 
agent $j$ is governed by the previous states of all $N$ agents. It can also be considered that $A_{i j} x_{i}(k)$ as a control input from the agent $i$ to the agent $j$ for $i \neq j$.

Proposition 2.6 In what follows is the detailed bibliographic review of DN methods which have been explained comprehensively in Table 2.10. The recent references have been explained and others have been cited in the Table. In the end, [69] has been considered using distributed networked control system over a lossy communication as a particular case.

\subsection{Mathematical Design in TRACK-TO-Track FU- SION}

Track fusion (TF)-based list of publications are classified in Table 2.11. Some recent publications in this area are as follows. Track fusion measurement is given in [18]. Performance of various track-to-track fusion algorithms from aspects of fusion accuracy, feedback and process noises are treated in [263]. Perform track fusion optimally for a multiple-sensor system with a specific processing architecture is treated in [295]. Other work cited in Table 2.11 are [338], [15, 22, 23, 24], [16], [17], [19], [21], [20], [71], [72], [73]-[74], [75]-[76], [77], [78]-[79], [124], [126], [260], [261, 262], [264, 265], [266], [296], [303], [305] and [306].

Proposition 2.7 In what follows is the detailed bibliographic review of TF-based methods which have been explained comprehensively in Table 2.11. The recent references have been explained and others have been cited in the Table. 
Table 2.10: DNs

\begin{tabular}{|c|c|}
\hline & \\
\hline $\begin{array}{l}\text { Worked control system (DNCS) with multiple nodes } \\
\text { te filtering algorithms for estimating states of a DNCS } \\
\text { I algorithm over sensor networks, consensus filter used to } \\
\text { ient statistics to neighbors and estimate global } \\
\text { tion and unsupervised clustering, first step in } \\
\text { alysis } \\
\text { r diffusion of local sufficient statistics over } \\
\text { ion of multiple sensor data to networks } \\
\text { ted state estimation against false data injection } \\
\text { consisting of a set of spatially scattered sensors } \\
\text { fading wireless channels }\end{array}$ & $\begin{array}{r}{[99]} \\
{[132]} \\
{[154]} \\
{[155]} \\
{[192]} \\
{[196]}\end{array}$ \\
\hline
\end{tabular}


Table 2.11: Mathematical Design in Track-to-Track Fusion

\begin{tabular}{l|r}
\hline \hline Track-to-Track Fusion Approaches & References \\
\hline - Track fusion with information filter & {$[338]$} \\
- Track fusion optimality with ML & {$[16]$} \\
- Two track estimates cross-covariance & {$[17]$} \\
- Track fusion local estimate dependency & {$[18]$} \\
- Track fusion measurement & {$[19]$} \\
- Track fusion multi-sensor algorithm & {$[21]$} \\
- Track fusion cross-covariance with independent noises & {$[20]$} \\
- Steady-state fusing problem & \\
- Steady state fused covariance for hierarchical track fusion architecture & {$[71]$} \\
with feedback & {$[72]$} \\
- Cross-covariance of the local track & {$[73]-[74]$} \\
- Weighted covariance state-vector Track fusion & {$[75]-[76]$} \\
- Pseudo-measurement state-vector Track fusion & {$[77]$} \\
- Steady state fused covariance matrix & {$[78]-[79]$} \\
- Various architectures for track association and fusion & {$[124]$} \\
- Fused estimate communicated to a central node to be used for some task & \\
- Track-to-track fusion algorithm, optimal in the sense of ML for more & {$[126]$} \\
than 2 sensors & {$[260]$} \\
- Measurement Fusion and State vector track fusion & {$[261][262]$} \\
- State vector track fusion with pseudo-measurement & \\
- Performance of various track-to-track fusion algorithms from aspects of & {$[263]$} \\
fusion accuracy, feedback and process noises & {$[264][265]$} \\
- Fuse state vectors using Weighted Covariance (WC) & {$[266]$} \\
- Weighted covariance algorithm turns out to be a ML estimate & \\
- Perform track fusion optimally for a multiple-sensor system with a & {$[295]$} \\
specific processing architecture & {$[296]$} \\
- Track-to-track fusion for multi-sensor data fusion & {$[303]$} \\
- Common process noise on the two-sensor fused-track covariance & {$[305]$} \\
- Track association and track fusion with non-deterministic target dynamics & \\
- Comparison of two-sensor tracking methods based on state vector fusion & {$[306]$} \\
and measurement fusion & \\
\hline
\end{tabular}




\subsection{DC-BASED Estimation}

DC-based estimation list of publications are classified in Table 2.12. Some recent work in this area is as follows. Recent work [32] is based on consensus Iterations. Distributed EM algorithm over sensor networks, consensus filter used to diffuse local sufficient statistics to neighbors and estimate global sufficient statistics in each node are the subject of [97]. A novel state estimation algorithm for linear stochastic systems, proposed on the basis of overlapping system decomposition, implementation of local state estimators by intelligent agents, application of a consensus strategy providing the global state estimates are detailed in [110]. Consensus-based distributed approached Kalman filters for linear systems $[121,122]$. Other publications cited in Table 2.12 are [28], [30], [31, 180], [33], [80], [10], [99], [102], [103], [104], [111], [112, 113], [118], [209], [210], [222], [243], [322], [327], [328] and [320] repectively.

Remark 2.9.1 In the paper [97], the number of Gaussian components is given. In the next step, distributed unsupervised clustering approach is used to select the number of Gaussian components, or it can use a distributed algorithm to estimate this number and run EM algorithm simultaneously. A well-fitted approach to this integration is the one proposed in [311]. The proposed distributed EM algorithm in the paper [97] handles this difficulty through estimating the global sufficient statistics using local information and neighbors local information. It calculates the local sufficient statistics in the E-step as usual first. Then, it estimates the global sufficient statistics. Finally, it updates the parameters in the M-step using the estimated global sufficient statistics. 
The estimation of global sufficient statistics is achieved by using an average consensus filter. The consensus filter can diffuse the local sufficient statistics over the entire network through communication with neighbor nodes [27, 28, 312] and estimate the global sufficient statistics using local information and neighbors local information. By using the estimated global sufficient statistics, each node updates the parameters in the M-step in the same way as in the standard EM algorithm. Because the consensus filter only requires local communication, that is, each node only needs to communicate with its neighbors and gradually gains global information, this distributed algorithm is scalable. It is shown that the equations of parameter estimation in this algorithm are not related to the number of sensor nodes. Thus, it is also robust. Failures of any nodes do not affect the algorithm performance given the network is still connected. Eventually, the estimated parameters can be accessed from any nodes in the network. In this paper, section, we a network of $M$ sensors is considered, each of which has $N_{m}$ data observations $y_{m, n}\left(m=1, \ldots, M, n=1, \ldots ., N_{m}\right.$. The environment is assumed to be a Gaussian mixture setting with $K$ mixture probabilities $\alpha_{m, k},(k=1, \ldots ., K)$. The unobserved state is denoted as $z$ and $z_{k}$ represents $z=k$. For each unobserved state $z_{k}$, observation $y_{m, n}$ follows a Gaussian distribution with mean $\mu_{k}$ and variance $\Sigma_{k}$ :

$$
p\left(y_{m, n} \mid \mu_{k}, \Sigma_{k}\right)=\frac{1}{\sqrt{2 \pi}\left\|\Sigma_{k}\right\|^{\frac{1}{2}}} e^{-\frac{1}{2}\left(y_{m, n}-\mu_{k}\right)^{T} \Sigma_{k}^{-1}\left(y_{m, n}-\mu_{k}\right)}
$$

The Gaussian mixture distribution for observation $y_{m, n}$ is:

$$
p\left(y_{m, n} \mid \theta\right)=\sum_{k=1}^{K} \alpha_{m, k} p\left(y_{m, n} \mid \mu_{k}, \Sigma_{k}\right)
$$


where $\theta$ is the set of the distribution parameters to be estimated $\theta=\left\{\alpha_{m, k}, \mu_{k}, \Sigma_{k} ; k=\right.$ $1, \ldots ., K, m=1, \ldots ., M\}$.

Proposition 2.8 In what follows is the detailed bibliographic review of DC-based estimation methods which have been explained comprehensively in Table 2.12. The recent references have been explained and others have been cited in the Table. In the end, [97] has been considered using Gaussian components.

\section{$2.10 \mathrm{DPF}$}

A DPF list of publications are classified in Table 2.13. Some recent work in this area is described as follows. A novel framework for delay-tolerant particle filtering, with delayed OOSM is treated in [137]. A number of heuristic metrics to estimate the utility of delayed measurements is proposed in [149]. Other recent publication in this area cited in Table 2.13 are [118], [133], [134], [135, 136], [144], [145], [146], [146], [148], [150], [181], [198], [235], [236], [250], [251], [301] and [302].

Proposition 2.9 In what follows is the detailed bibliographic review of DPF methods which have been explained comprehensively in Table 2.13. The recent references have been explained and others have been cited in the Table. 
Table 2.12: DC-Based Estimation

Design Approaches used in DC

- Iterative consensus protocols

- Local average consensus algorithms

- Based on consensus strategies

- Based consensus Iterations

- Converge Speed of consensus strategies

- Dynamic consensus problems regarding fusion of the measurements and covariance information with consensus filters

- Using Standard KF locally with a consensus step

- Distributed EM algorithm over sensor networks, consensus filter used to diffuse local sufficient statistics to neighbors and estimate global sufficient statistics in each node

- Distributed EM algorithm over SNs, consensus filter used to diffuse local sufficient statistics

- Consensus filter diffusion of local sufficient statistics over the entire network through communication with neighbor nodes

- Consensus-based distributed linear filtering problem

- The interaction between the consensus matrix and the Kalman gain for scalar systems

- KF with a consensus filter, ensuring estimates asymptotically converge to the same value

- Novel state estimation algorithm for linear stochastic systems, proposed on the basis of overlapping system decomposition, implementation of local state estimators by intelligent agents, application of a consensus strategy providing the global state estimates

- Average-consensus algorithm for $n$ measurements of noisy signals obtained from $n$ sensors in the form of a distributed low-pass filter

- Average-consensus algorithm for $n$ constant values

- Consensus-Based distributed implementation of the unscented particle filter

- Consensus-based distributed approached KFs for linear systems

- A message-passing version of the Kalman-Consensus Filter (KCF)

- A peer-to-peer (P2P) architecture of DKF that rely on reaching a consensus on estimates of local KFs

- Consensus-based suboptimum KF scheme

- Distributed filter that allows the nodes of a SN to track the average of $n$ sensor measurements

- DC-Based estimation for networks of agents, uncertain systems, jump Markov Systems and SN with delay

References 
Table 2.13: DPF

Design Approaches used in DPF
References

- Consensus-Based distributed implementation of the unscented particle filter (UPF)

- Particle filtering transformation into continuous representations

- Consensus-based, distributed implementation of the UPF

- Particle filter implementations using Gaussian approximations for the local posteriors

- A novel framework for delay-tolerant particle filtering, with delayed OOSM

- An approach that stores sets of particles for the last $l$ time steps, where $l$ is the predetermined maximum delay

- Markov chain Monte Carlo (MCMC) smoothing step for OOSM

- Approximate OOSM particle filter based on retrodiction(predicts backward)

- Also uses retrodiction (predicts backward), but employs the Gaussian particle filter

- Recent advances in particle smoothing, storage-efficient particle filter

- Proposed a number of heuristic metrics to estimate the utility of delayed measurements

- Proposed a threshold based procedure to discard uninformative delayed measurements, calculating their informativeness

- Optimal estimation using quantized innovations, with application to distributed estimation over SNs using Kalman-like particle filter

- SOl-Particle-Filter (SOI-PF) derived to enhance the performance of the distributed estimation procedure

- Problem of tracking a moving target in a multi-sensor environment DPFs

- Optimal fusion method, introduced to fuse the collected GMMs with different number of components

- Two distributed particle filters to estimate and track the moving targets in a WSN

- Updating the complete particle filter on each individual sensor nodes

- Out-of-sequence measurement processing for tracking ground target using PFs

- Comparison of the KF and PF based OOSM filtering algorithms

$[135][136]$ [302] 


\subsection{ST-Based Distributed Fusion Kalman Fil-}

\section{TER}

This section explains the ST-based distributed fusion Kalman filter, another categorization for DKF. A list of publications is this regard is classified in Table 2.14. Some of the recent work in this area is as follows. Self-tuning decoupled fusion Kalman predictor is proposed in [160] and self-tuning weighted measurement Kalman filter is included in [161]. Self-tuning measurement system using the correlation method, can be viewed as the least-squares (LS) fused estimator and found in [285]. Self-tuning distributed (weighed) measurement fusion Kalman filters is shown in [292, 293, 294]. Other recent publication in this area cited in Table 2.14 are [157], [159], [182], [184, 185], [189], [190], [239], [245], [246], [258], [286]-[289], [290] and [291].

Remark 2.11.1 For self-tuning decoupled fusion Kalman predictor, the following multisensor linear discrete time-invariant stochastic system is considered in the paper [308]:

$$
\begin{aligned}
x(t+1) & =\Phi x(t)+\Gamma w(t) \\
y_{i}(t) & =H_{i} x(t)+v_{i}(t), i=1, \ldots ., L
\end{aligned}
$$

where $x(t) \in \Re^{n}, y_{i}(t) \in \Re^{m_{i}}, w(t) \in \Re^{r}$ and $v_{i}(t) \in \Re^{m_{i}}$ are the state, measurement, process and measurement noises of the ith sensor subsystem, respectively, and $\Phi$, $\Gamma$ and $H_{i}$ are constant matrices with compatible dimensions.

Proposition 2.10 In what follows is the detailed bibliographic review of ST-based dis- 
Table 2.14: ST-Based Distributed Fusion Kalman Filter

ST Design Approaches

- Multi-sensor systems with unknown model parameters and noise variances, by the information matrix approach, the ST distributed state fusion information filter is presented - ST distributed state fusion Kalman filter with weighted covariance approach

- ST decoupled fusion Kalman predictor

- ST weighted measurement Kalman filter

- Multi-sensor systems with unknown noise variances,

a new ST weighted measurement fusion Kalman filter is presented, which has asymptotic global optimality

- Weighted ST state fusion filters

- Sign of Innovation- Particle Filter (SOI-PF) improves the tracking performance when the target moves according to a linear and a gaussian model

- Efficiency of the SOI-PF in a nonlinear and a non gaussian case, considering a jump-state Markov model for the target trajectory

- ST information fusion reduced-order Kalman predictor with a two-stage fusion structure based on linear minimum variance

- Optimal ST smoother

- Optimal ST fix-lag smoother

- A new convergence analysis method for ST Kalman Predictor

- ST measurement system using the correlation method, can be viewed as the least-squares (LS) fused estimator

- ST filtering for systems with unknown model and/or noise variances

- ST distributed state fusion Kalman estimators

- ST distributed (weighed) measurement fusion Kalman filters References

\begin{tabular}{r|r} 
References \\
\\
{$[157]$} \\
{$[159]$} \\
{$[160]$} \\
{$[161]$} \\
\\
{$[182]$} \\
{$[184][185]$} \\
\\
{$[189]$} \\
{$[190]$} \\
{$[239]$} \\
{$[245]$} \\
{$[246]$} \\
{$[258]$} \\
{$[285]$} \\
{$[286]-[289]$} \\
{$[290][291]$} \\
{$[292][293][294]$} \\
\end{tabular}


tributed KF methods which have been explained comprehensively in Table 2.14. The recent references have been explained and others have been cited in the Table. In the end, [308] has been considered using decoupled fusion Kalman predictor. 


\section{Approximate Distributed Esti-}

\section{MATION}

\subsection{AN OVERVIEW}

In this chapter, we have discussed approximate distributed estimation, where we have derived the distributed estimation for different prior cases with the help of Bayesianbased Forward Backward (FB) Kalman filter.

\subsection{INTRODUCTION}

Distributed and decentralized estimations have been the point of attraction in the past with a large associated literature. When tackling the distributed structure, problems do encounter regarding fusion of the data coming from various sensor of the plant or $\underline{\text { network. Data fusion techniques combine data from multiple sensors and related infor- }}$ $\underline{\text { mation to achieve more specific inferences than could be achieved by using a single, }}$ independent sensor. The classic work of Rao and Durrant-Whyte [381] 
presents an approach to decentralized Kalman filtering which accomplishes globally optimal performance in the case where all sensors can communicate with each other. Further, this design failed gracefully, as individual sensors are removed from the network due to its distributed design. Sensor noises of converted systems crosscorrelated, whilst original system independent is shown in [338]-[339]. Sensor noises of converted system cross-correlated, whilst original system also correlated is presented in [335]. Centralized fusion center, expressed by a linear combination of the local estimates is pictured in [336]. No centralized fusion center, but algorithm highly resilient to lose one or more sensing nodes is shown in [337]. Discrete smoothing fusion with ARMA signals is shown in [332]. Linear minimum variance with information fusion filter is shown in [333][334]. A dense attention has been devoted to multi-sensor data fusion for both military and civilian applications. For civilian applications, monitoring of manufacturing processes, robotics, medical applications/environmental monitoring are considered. For military applications, target recognition, guidance for autonomous vehicles and battle field surveillance are considered.

Estimation problem has also been dealt with consensus algorithms. Consensus problems [340], [341] and their special cases have been the subject of intensive studies by several researchers [342], [343], [344], [345], [346], [347], [348] in the context of formation control, self-alignment, and flocking [349] in networked dynamic systems.

In distributed estimation and fusion, Kalman filtering is a fundamental tool, and it is an essential element to provide functionality particularly in sensor networks. An indepth comparison between the distributed Kalman filter and the existing decentralized 
sensor fusion algorithms both with and without fusion centers are presented in [350], [351], [352], [353] respectively.

In this paper, we have derived an approximate distributed estimation for different prior cases for dynamic systems, with the help of Bayesian-based FB Kalman filter, The estimation is derived on a distributed networked control system [330]. Then, to reduce the time complexity, upper bound and lower bound methods for time complexity reduc$\underline{\text { tion have been derived on all three cases of prior knowledge. After achieving estimates, }}$ we have used a data fusion technique to consider it for a distributed structure. The proposed scheme is then validated on a network structure of a rotational drive-based electro-hydraulic system, where various types of faults were introduced, and then different fault profile data are considered for the evaluation of the proposed scheme.

The remainder of this paper is structured as follows. Problem formulation is described in Section II. The Bayesian-based FB Kalman filter with complete prior information is derived and discussed in Section III, the Bayesian-based FB Kalman filter without prior information is derived and discussed in Section IV, followed by derivation of Bayesian-based FB Kalman filter with incomplete prior information in Section V. Evaluation and testing is made in Section VI. Finally some conclusion is described in Section VII.

\subsection{PROBLEM Formulation}

Consider a distributed control system as in [330] consisting of $N$ agents, in which there is no communication loss. The discrete-time linear dynamic model of the agent $j$ can 
be described as:

$$
x_{j}(k+1)=\sum_{i=1}^{N} A_{i j} x_{i}(k)+G_{j} w_{j}(k)
$$

where $k \in \mathbf{Z}^{+}, x_{j}(k) \in \mathbf{R}^{n_{x}}$ is the state of the agent $j$ at time $k, w_{j}(k) \in R^{n_{w}}$ is a white noise process, $A_{i j} \in \mathbf{R}^{n_{x} \times n_{x}}$, and $G_{j} \in R^{n_{x} \times n_{w}}$. Hence, the state of the agent $j$ is governed by the previous states of all $N$ agents. We can also consider $A_{i j} x_{i}(k)$ as a control input from the agent $i$ to the agent $j$, where $i \neq j$.

Now consider a distributed networked control system (DNCS), in which agents communicate with each other over a lossy communication channel. We assume an erasure channel between a pair of agents. At each time $k$, a packet sent by the agent $i$ is correctly received by the agent $j$ with probability $p_{i j}$. We form a communication matrix $P_{c o m}=\left[p_{i j}\right]$. Let $Z_{i j}(k) \in\{0,1\}$ be a Bernoulli random variable, such that $Z_{i j}(k)=1$ if a packet sent by the agent $i$ is correctly received by the agent $j$ at time $k$, otherwise, $Z_{i j}(k)=0$. Since there is no communication loss within an agent, $p_{i i}=$ 1 and $Z_{i i}(k)=1$ for all $i$ and $k$. For each $(i, j)$ pair, $\left\{Z_{i j}(k)\right\}$ are i.i.d. (independent identically distributed) random variables such that $P\left(z_{i j}(k)=1\right)=p_{i j}$ for all $k$; and $Z_{i j}(k)$ are independent from $Z_{l m}(k)$ for $l \neq i$ or $m \neq j$. Then we can write the dynamic model of the agent $j$ under lossy links as:

$$
x_{j}(k+1)=\sum_{i=1}^{N} Z_{i j}(k) A_{i j} x_{i}(k)+G_{j} w_{j}(k)
$$

where $Z_{i j}$ is a random Bernoulli variable. 
Let $x(k)=\left[x_{1}(k)^{T}, \ldots, x_{N}(k)^{T}\right]^{T}$ and $w(k)=\left[w_{1}(k)^{T}, \ldots, w_{N}(k)^{T}\right]^{T}$, where $y^{T}$ is a transpose of $y$. Let $\bar{A}_{i j}$ be a $N_{n_{x}} \times N_{n_{x}}$ block matrix. The entries of $\bar{A}_{i j}$ are all zeros except the $(j, i)-t h$ block is $A_{i j}$. For example, when $N=2$.

$$
\bar{A}_{12}=\left[\begin{array}{ll}
0_{n x} & 0_{n x} \\
A_{12} & 0_{n x}
\end{array}\right]
$$

where $0_{n x}$ is a $n_{x} \times n_{x}$ zero matrix. Then the discrete-time linear dynamic model of the DNCS with lossy links can be represented as following:

$$
x(k+1)=\left(\sum_{i=1}^{N} \sum_{j=1}^{N} Z_{i j}(k) \bar{A}_{i j}\right) x(k)+G w(k)
$$

where $G$ is a block diagonal matrix of $G_{1}, \ldots, G_{N}$. For notational convenience, we introduce a new index $n \in 1, \ldots, N_{2}$ such that $i j$ is indexed by $n=N(i-1)+j$. With this new index $n$, the dynamic model (3.3) can be rewritten as:

$$
x(k+1)=\left(\sum_{n=1}^{N^{2}} Z_{n}(k) \bar{A}_{n}\right) x(k)+G w(k)
$$

By letting $A(k)=\left(\sum_{n=1}^{N^{2}} Z_{n}(k) \bar{A}_{n}\right)$ we see that (3.4) is a time-varying linear dynamic model:

$$
x(k+1)=A(k) x(k)+G w(k)
$$

Until now we have assumed that $\bar{A}_{n}$ is fixed for each $n$. Now suppose a more 
general case where the matrix $A$ is time-varying and its values are determined by the communication link configuration $Z(k)=\left[Z_{1}(k), \ldots, Z_{N^{2}}(k)\right]^{T}$. Hence, $A$ is a function of $Z(k)$ and this general case can be described as:

$$
x(k+1)=A(Z(k)) x(k)+G w(k)
$$

The dynamic model (3.6) or (3.4) is a special case of the linear hybrid model or a jump linear system [355] since $A(k)$ takes an element from a set of a finite number of matrices. We will call the dynamic model (3.4) as the "simple" DNCS dynamic model and (3.6) as the "general" DNCS dynamic model.

In the following sections, we will derive Kalman filter fusion with cases of prior information, and their modifications which can bound the covariance matrices [330]. The Bayesian-based FB Kalman filter is expressed as follows (See Equation (3.7-3.15)), where the simple Bayesian-based optimal Kalman filter is expressed in [329], where the basic version of Bayesian-based Kalman filter is derived, from which the Bayesianbased FB Kalman and its versions for different prior knowledge have then been derived 
and formulated here.

$$
\begin{aligned}
& \text { Forward Run: For }(k=0 ; k<T ;+k) \\
& R_{e, i}=R_{i}+H_{k} P_{k+1 / k} H_{k}^{*} \\
& \widehat{K}_{f, i}=F_{k+1 / k} \widehat{P}_{k+1 / k} H_{k}^{T}\left(H_{k} \widehat{P}_{k / k-1} H_{k}^{T}+R_{e, i}^{-1}\right) \\
& \widehat{x}_{k / k}^{M A P}=\widehat{x}_{k+1 / k}+\widehat{K}_{f, i}\left(y_{k}-H_{k} \widehat{x}_{k+1 / k}\right) \\
& \widehat{x}_{k+1 / k}=F_{k} \widehat{x}_{k+1 / k} \\
& \widehat{P}_{k+1 / k}=F_{k+1 / k} P_{k+1 / k} F_{k+1 / k}^{T}+G_{i} Q G_{i}^{*} \\
& -\widehat{K}_{p, i} R_{e, i} \widehat{K}_{p, i}^{*} \\
& \widehat{P}_{k / k}=\widehat{P}_{k+1 / k}-F_{k / k+1} \widehat{K}_{k} H_{k} \widehat{P}_{k+1 / k}
\end{aligned}
$$

Backward Run: For $(k=T-1 ; t \geq 0 ;-k)$

$$
\begin{aligned}
& \widehat{J}_{k-1 / T}=\widehat{P}_{k-1 / T} F_{k}^{T} \widehat{P}_{k-1 / T}^{-1} \\
& \widehat{x}_{k-1 / T}=\widehat{x}_{k-1 / k-1}^{i}+\widehat{J}_{k-1}\left(\widehat{x}_{k-1 / T}-\widehat{x}_{k-1 / k}\right) \\
& \widehat{P}_{k-1 / T}=\widehat{P}_{k-1 / k-1} \\
& +\widehat{J}_{k-1}\left(\widehat{J}_{k-1 / T}-\widehat{P}_{k-1} / k\right) J_{k-1}^{\prime}
\end{aligned}
$$

where $R_{e, i}$ is the covariance matrix of residual, $P_{k+1 / k}$ is the a-posteriori error covariance matrix, $H_{k}$ is the observation model, $\hat{K}_{f, i}$ is the system gain, $Q$ is the covariance of the process noise, and $F_{k}$ is the state-transition model for each time-step $k$.

It should be noted that smoother is being employed here to reduce noise effect and 
have more clear results in the approximate estimation of various prior information versions due to its nature of choosing the most refined covariance error matrix $P_{k}$ from the last iteration instant of forward run and considering it as the first iteration in the backward run. Note that it is the designers choice whether to use smoothing equations or not. For example, during an on-line analysis, the Kalman smoother will give estimates only after the end of the experiment, which may not be acceptable. But for an off-line analysis, getting the estimates after the experiment may not matter.

\subsection{BAYESIAN-BASED FB KALMAN FILTER FUSION With COMPLETE PRIOR INFORMATION}

In this section, generalized version of Kalman filter is presented with complete prior information. Consider the generalized DNCS dynamic model (3.6) where $w(k)$ is a Gaussian noise with zero mean and covariance $Q$, and measurement model (3.16) where $y(k) \in R^{n_{y}}$ is a measurement at time $t, C \in \mathbf{R}^{n_{y} \times N_{n_{x}}}$ and $\nu(k)$ is a Gaussian noise with zero mean and covariance $k$.

$$
y(k)=C x(k)+\nu(k)
$$

The following theorem presents the Bayesian-based FB Kalman filter with complete prior information: 


\section{Theorem 3.1}

Forward Run: For $(k=0 ; k<T ;+k)$

$$
\begin{aligned}
& \widehat{x}_{k / k}=F_{k} \bar{x}_{k}+K_{p, k}\left[y_{i}-H_{k} \bar{x}_{k+1 / k}-\bar{\nu}\right] \\
& \widehat{x}_{k+1 / k}=F_{k} \widehat{x}_{k+1 / k}+K_{p, k} \nu_{k} \\
& \widehat{R}_{e, k}=R_{k}+H_{k} P_{k+1 / k} H_{k}^{*}+H C_{x v}+\left(H C_{x v}\right)^{\prime} \\
& K_{k}=\left(F_{k} P_{k+1 / k} H^{*}+G_{k} S_{k}\right)\left(H_{k} P_{k / k} H_{k}^{*}+R_{e, k}\right)^{-1} \\
& \widehat{P}_{k+1 / k}=F_{k} P_{k+1 / k} F_{k}^{*}+G Q_{i} G^{*} \\
& -F_{k+1 / k} K_{p, k} R_{e, k} K_{p, k}^{*} \\
& \widehat{P}_{k / k}=F_{k} P_{k+1 / k} F_{k}^{*}-K_{k} H_{k} P_{k+1 / k}
\end{aligned}
$$

Backward Run: For $(k=0 ; k<T ;+k)$

$$
\begin{aligned}
& \widehat{J}_{k-1 / T}=\widehat{P}_{k-1 / T} F_{k}^{T} \widehat{P}_{k-1 / T}^{-1} \\
& \widehat{x}_{k-1 / T}=\widehat{x}_{k-1 / k-1}^{i}+\widehat{J}_{k-1}\left(\widehat{x}_{k-1 / T}-\widehat{x}_{k-1 / k}\right) \\
& \widehat{P}_{k-1 / T}=\widehat{P}_{k-1 / k-1} \\
& +\widehat{J}_{k-1}\left(\widehat{J}_{k-1 / T}-\widehat{P}_{k-1} / k\right) J_{k-1}^{\prime}
\end{aligned}
$$


where $S_{k}$ is the covariance of $\tilde{y}_{k}$. The error covariance and the gain matrices have the following alternative forms (See Eqns. (3.26) and (3.27)):

$$
\begin{aligned}
P & =F P_{k+1 / k+1} F^{\prime}+K R_{e, k} K^{\prime}-F P K^{\prime}-\left(F B K^{\prime}\right)^{\prime} \\
K & =\left(F_{k} P_{k+1 / k} H^{*}+P_{k / k}\right)\left(K R_{e, k} K+H P_{k / k}\right)^{-1}
\end{aligned}
$$

where $B_{k}$ is the control-input model.

Proof. For linear estimation of $x$ using data $y$ with linear model $y=H x+\nu$, the prior information consists of $\bar{x}$ and $\bar{\nu}$, and $C_{x}=\operatorname{cov}(x), C_{v}=\operatorname{cov}(v)$, and $C_{x v}=\operatorname{cov}(x, v)$. When we talk about prior information, we mean prior information about $x$, that is $\bar{x}$, $C_{x}$, and $C_{x, v}$

For dynamic case, as in Kalman filter,

$$
\begin{aligned}
\hat{x}_{k / k} & =\mathbf{E}^{*}\left[x_{k} \mid y^{k}\right]=\left[\bar{x}_{k} \mid y^{k}\right] \\
& =\bar{x}_{k}+C_{x_{k}} y^{k} C^{+} y^{k}\left(y^{k}-\bar{y}^{k}\right), \bar{x}_{k}=\mathbf{E}\left[x_{k}\right] \\
P_{k / k} & =\operatorname{MSE}\left(\hat{x}_{k / k}\right)={ }^{\triangle} \mathbf{E}\left[\left(x_{k}-\hat{x}_{k / k}\right)\left(x_{k}-\hat{x}_{k / k}\right)^{\prime}\right] \\
& =C x_{k}-C x_{k} y^{k} C_{y^{k}}^{+} C_{x_{k}}^{\prime} y^{k}
\end{aligned}
$$

With few exceptions, however, it is unrealistic since its computational burden increases rapidly with time (method for decreasing time computation complexity is applied in the 
next section using modified kalman filter functions of upper bound and lower bound).

$$
\begin{aligned}
& \hat{x}_{k / k}=E^{*}\left[x_{k} \mid y^{k}\right]=\mathbf{E}^{*}\left[x_{k} \mid y_{k}, y^{k-1}\right]=\hat{x}_{k / k-1}+K_{k} \bar{y}_{k / k-1} \\
& P_{k / k}=\operatorname{MSE}\left(\hat{x}_{k / k}\right)=\operatorname{MSE}\left(\hat{x}_{k / k-1}\right)-K_{k} C \bar{y}_{k / k-1} K_{k}^{\prime}
\end{aligned}
$$

Let $A=P_{k / k}$ and $F_{k}=\zeta$. Equation (3.27) follows from the following:

$$
\begin{aligned}
& \left(\zeta P H^{\prime}+A\right)(C+H A)^{-1} \\
= & \left\{\zeta \left[C_{x}-\left(C_{x} H^{\prime}+A\right)\left(H C_{x} H^{\prime}+C+H A+(H A)^{\prime}\right)^{-1}\right.\right. \\
\cdot & \left.\left.\left(C_{x} H^{\prime}+A\right)^{\prime}\right] H^{\prime}+A\right\}(C+H A)^{-1} \\
= & \left(\zeta C_{x}+H^{\prime}+A\right)\left[I-\left(H C_{x} H^{\prime}+C+H A+(H A)^{\prime}\right)^{-1}\right. \\
\cdot & \left.\left(H C_{x} H^{\prime}+(H A)^{\prime}\right)\right](C+H A)^{-1} \\
= & \left(\zeta C_{x} H^{\prime}+A\right)\left(H C_{x} H^{\prime}+C+H A+(H A)^{\prime}\right)^{-1} \\
\cdot & (C+H A)(C+H A)^{-1} \\
= & \left(\zeta C_{x} H^{\prime}+A\right)\left(C_{y}+H A\right)^{-1}
\end{aligned}
$$

\subsubsection{MOdified Filter With COMPLETE PRIOR INFORMATION}

Based on general DNCS dynamic model (3.6), where $Z(k)$ is independent from $Z(t)$ for $t \neq k$, we derive an optimal linear filter.

The following terms are defined to describe the modified Bayesian-Based FB Kalman 
filter.

$$
\begin{aligned}
\widehat{x}_{k / k} & =\mathbf{E}\left[x(k) \mid y_{k}\right] \\
P(k \mid k) & =\mathbf{E}\left[e(k) e(k)^{T} \mid y_{k}\right] \\
\widehat{x}(k+1 \mid k) & =\mathbf{E}\left[x(k+1) \mid y_{k}\right] \\
P(k+1 \mid k) & =\mathbf{E}\left[e(k+1 \mid k) e(k+1 \mid k)^{T} \mid y_{k}\right] \\
J(k-1 \mid T) & =\mathbf{E}\left[J(k-1 \mid T) \mid P_{k / k}\right] \\
\widehat{x}(k-1 \mid T) & =\mathbf{E}\left[e(k-1 \mid T) \mid y_{k}\right] \\
P(k-1 \mid T) & =\mathbf{E}\left[e(k-1 \mid T) e(k-1 \mid T)^{T} \mid y_{k}\right]
\end{aligned}
$$

where $y_{k}=\{y(t): 0 \leq t \leq k\}, e(k \mid k)=x(k)-\widehat{x}(k \mid k)$, and $e(k+1 \mid k)=x(k+1)-$ $\widehat{x}(k+1 \mid k)$.

Suppose that we have estimates $\widehat{x}(k \mid k)$ and $P(k \mid k)$ from time $k$. At time $k+1$, a new measurement $y(k+1)$ is received and our goal is to estimate $\widehat{x}(k+1 \mid k+1)$ and $P(k+1 \mid k+1)$ from $\widehat{x}(k \mid k), P(k \mid k)$ and $y(k+1)$. First, we compute $\widehat{x}(k+1 \mid k)$ and $P(k+1 \mid k)$.

$$
\begin{aligned}
\widehat{x}(k+1 \mid k) & =\mathbf{E}\left[x(k+1) \mid y_{k}\right] \\
& =\mathbf{E}\left[A(Z) x(k)+G \omega(k) \mid y_{k}\right] \\
& =\widehat{A} \widehat{x}(k \mid k)
\end{aligned}
$$


where

$$
\widehat{A}=\sum_{z \in Z} p_{z} A(z)
$$

is the expected value of $A(Z)$. Here $p_{z}=P(Z=z)$, and $\mathcal{Z}$ is a set of all possible communication link configurations.

The prediction covariance can be computed as:

$$
\begin{aligned}
P(k+1 \mid k)= & \mathbf{E}\left[e(k+1 \mid k) e(k+1 \mid k)^{T} \mid y_{k}\right] \\
= & G Q G^{T}+\sum_{z \in Z} p_{z} A(z) P(k \mid k) A(z)^{T} \\
& -K_{p, k} R_{e, k} K_{p, k}^{*}+\sum_{z \in Z} p_{z} A(z) \widehat{x}(k \mid k) \widehat{x}(k \mid k)^{T} \\
& \times(A(z)-\widehat{A})^{T}
\end{aligned}
$$

Given $\widehat{x}(k+1 \mid k)$ and $P(k+1 \mid k), \widehat{x}(k+1 \mid k+1)$ and $P(k+1 \mid k+1)$ are computed as in the standard Kalman filter (See Eqn. (3.32) and (3.33)).

$$
\begin{aligned}
\widehat{x}(k+1 \mid k+1)= & F_{k} \widehat{x}(k+1 \mid k)+K(k+1)(y(k+1) \\
& -H \widehat{x}(k+1 \mid k))-\nu_{i} \\
P(k+1 \mid k+1)= & F_{k} P(k+1 \mid k) F_{k}^{*} \\
& -F_{k / k-1} K_{k}(k+1) H P(k+1 \mid k)
\end{aligned}
$$

where $K(k+1)=\left(F P k+1 \mid k H^{T}+G S\right)\left(H P k \mid k H^{T}+R\right)^{-1}$. 


\subsubsection{APPROXIMATING THE FILTER FOR COMPLETE PRIOR INFOR-}

\section{MATION}

The modified KF proposed in Section 3.4.1 for the general DNCS is an optimal linear filter but the time complexity of the algorithm can be exponential in $N$ since the size of $\mathcal{Z}$ is $O\left(2^{N(N-1)}\right)$ in the worst case, i.e., when all agents can communicate with each other. In this section, we describe two approximate Kalman filtering methods for the general DNCS dynamic model (6) which are more computationally efficient than the modified KF by avoiding the enumeration over $\mathcal{Z}$. Since the computation of $P(k+1 \mid k)$ is the only time-consuming process, we propose two filtering method which can bound $P(k+1 \mid k)$. We use the notation $A \geq 0$ if $A$ is a positive definite matrix and $\mathrm{A} \geq 0$ if $A$ is a positive semi-definite matrix.

\section{Lower-Bound KF: Complete Prior Information Case}

The lower-bound KF (lb-KF) is the same as the modified KF described in Section III, except we approximate $P(k+1 \mid k)$ by $\underline{P}(k+1 \mid k)$ and $P(k \mid k)$ by $\underline{P}(k \mid k)$. The covariances are updated as:

$$
\begin{aligned}
\underline{P}(k+1 \mid k)= & \widehat{\widehat{A}} \underline{P}(k \mid k) \widehat{A}^{T}+G Q G^{T} \\
& -\underline{K}_{p, k} \underline{R}_{e, k} \underline{K}_{p, k} \\
\underline{P}(k+1 \mid k+1)= & F_{k} \underline{P}(k+1 \mid k) \\
& -F_{k / k-1} \underline{K}(k+1) H_{k} \underline{P}(k+1 \mid k)
\end{aligned}
$$


where $\widehat{A}$ is the expected value of $A(Z)$ and $\underline{K}(k+1)=F_{k+1 / k} \underline{P}(k+1 \mid k) H^{T}\left(H_{k} \underline{P}(k+\right.$ $\left.1 \mid k) H_{k}^{*}+R\right)^{-1}$. Notice that $\widehat{A}$ can be computed in advance and the $\mathrm{lb}-\mathrm{KF}$ avoids the enumeration over $\mathcal{Z}$.

Lemma 3.1 If $\underline{P}(k \mid k) \leq P(k \mid k)$, then $\underline{P}(k+1 \mid k) \leq P(k+1 \mid k)$.

Proof. Using (3.31), we have

$$
\begin{aligned}
P(k+1 \mid k)-\underline{P}(k+1 \mid k) & =\mathbf{E}\left[A(Z) P(k \mid k) A(Z)^{T}\right] \\
& +\mathbf{E}\left[A(Z) \widehat{x}(k \mid k) \widehat{x}(k \mid k)^{T} A(Z)^{T}\right] \\
& -\widehat{A} \widehat{x}(k \mid k) \widehat{x}(k \mid k)^{T} \widehat{A}^{T}-\widehat{A} \underline{P}(k \mid k) \widehat{A}^{T} \\
& -\underline{K}_{p, k} \underline{R}_{e, k} \underline{K}_{p, k}+K_{p, k} R_{e, k} K_{p, k} \\
& =P_{1}+P_{2}
\end{aligned}
$$

where $P_{1}=\mathbf{E}\left[A(Z) P(k \mid k) A(Z)^{T}\right]-\widehat{A} \underline{P}(k \mid k) \widehat{A}^{T}-\underline{K}_{p, k} \underline{R}_{e, k} \underline{K}_{p, k}$ and $P_{2}=$ $\mathbf{E}\left[A(Z) \widehat{x}(k \mid k) \widehat{x}(k \mid k)^{T} A(Z)^{T}\right]-\widehat{A} \widehat{x}(k \mid k) \widehat{x}(k \mid k)^{T} \widehat{A}^{T}+K_{p, k} R_{e, k} K_{p, k}$.

If $P_{1} \geq 0$ and $P_{2} \geq 0$, then $P(k+1 \mid k)-\underline{P}(k+1 \mid k) \geq 0$

$$
\begin{aligned}
P_{1} & =\mathbf{E}\left[A(Z) P(k \mid k) A(Z)^{T}\right]-\widehat{A} \underline{P}(k \mid k) \widehat{A}^{T}-\underline{K}_{p, k} \underline{R}_{e, k} \underline{K}_{p, k}^{*} \\
& -\widehat{A} P(k \mid k) \widehat{A}^{T}+\widehat{A} P(k \mid k) \widehat{A}^{T} \\
& =\mathbf{E}\left[A(Z) P(k \mid k) A(Z)^{T}\right]-\widehat{A} P(k \mid k) \widehat{A}^{T} \\
& +\widehat{A}(P(k \mid k)-\underline{P}(k \mid k)) \widehat{A}^{T}-\underline{K}_{p, k} \underline{R}_{e, k} \underline{K}_{p, k}^{*}
\end{aligned}
$$

Since $P(k \mid k)$ is a symmetric matrix, $P(k \mid k)$ can be decomposed into $P(k \mid k)=$ 
$U_{1} D_{1} U_{1}^{T}$, where $U_{1}$ is a unitary matrix and $D_{1}$ is a diagonal matrix. Hence,

$$
\begin{aligned}
P_{1} & =\mathbf{E}\left[\left(A(Z) U_{1} D_{1}^{1 / 2}\right)\left(A(Z) U_{1} D_{1}^{1 / 2}\right)^{T}\right] \\
& -\mathbf{E}\left[\left(A(Z) U_{1} D_{1}^{1 / 2}\right)\right] \mathbf{E}\left[\left(A(Z) U_{1} D_{1}^{1 / 2}\right)\right]^{T} \\
& +\widehat{A}(P(k \mid k)-\underline{P}(k \mid k)) \widehat{A}^{T}-\underline{K}_{p, k} \underline{R}_{e, k} \underline{K}_{p, k}^{*} \\
& =\operatorname{Cov}\left[\left(A(Z) U_{1} D_{1}^{1 / 2}\right]+\widehat{A}(P(k \mid k)-\underline{P}(k \mid k)) \widehat{A}^{T}\right. \\
& -\underline{K}_{p, k} \underline{R}_{e, k} \underline{K}_{p, k}
\end{aligned}
$$

where Cov $[H]$ denotes the covariance matrix of $H$. Since a covariance matrix is positive definite and $P(k \mid k)-\underline{P}(k \mid k) \geq 0$ by assumption, $P_{1} \geq 0 . P_{2}$ is a covariance matrix since $\widehat{x}(k \mid k) \widehat{x}(k \mid k)^{T}$ is symmetric, hence $P_{2} \geq 0$.

Lemma 3.2 If $\underline{P}(k+1 \mid k) \leq P(k+1 \mid k)$, then $\underline{P}(k+1 \mid k+1) \leq P(k+1 \mid k+1)$.

Proof. Here, we will use matrix inversion lemma which says that $(A+U C V)^{-1}=$ $A^{-1}-A^{-1} U\left(C^{-1}+V A^{-1} U\right)^{-1} V A^{-1}$ where $A, U, C$ and $V$ all denote matrices of the correct size. Applying the matrix inversion lemma to (3.33), we have $P(k+1 \mid k+1)=$ $\left(P(k+1 \mid k)^{-1}+C^{T} R^{-1} C\right)^{-1}$. Let $P=P(k+1 \mid k)$ and $\underline{P}=\underline{P}(k+1 \mid k)$. Then

$P \geq \underline{P} \Rightarrow P^{-1} \leq \underline{P}^{-1}$

and

$P^{-1}+C^{T} R^{-1} C \leq \underline{P}^{-1}+C^{T} R^{-1} C \Rightarrow\left(P^{-1}+C^{T} R^{-1} C\right)^{-1} \geq\left(\underline{P}^{-1}+C^{T} R^{-1} C\right)^{-1}$

and

$P(k+1 \mid k+1) \geq \underline{P}(k+1 \mid k+1)$

Finally, using Lemma 3.1, Lemma 3.2, and the induction hypothesis, we have the 
following theorem showing that the lb-KF maintains the state error covariance which is upper-bounded by the state error covariance of the modified KF.

Theorem 3.2 If the $l b-K F$ starts with an initial covariance $\underline{P}(0 \mid 0)$, such that $\underline{P}(0 \mid 0) \leq$ $P(0 \mid 0)$, then $\underline{P}(k \mid k) \leq P(k \mid k)$ for all $k \geq 0$.

\section{Upper-bound KF: Complete Prior Information Case}

Similar to the $l b-K F$, the upper-bound $K F(u b-K F)$ approximates $P(k+1 \mid k)$ by $\bar{P}(k+$ $1 \mid k)$ and $P(k \mid k)$ by $\bar{P}(k \mid k)$. Let $\lambda_{\max }=\lambda_{\max }(\bar{P}(k \mid k))+\lambda_{\max }\left(\widehat{x}(k \mid k) \widehat{x}(k \mid k)^{T}\right)$, where $\lambda_{\text {max }}(S)$ denotes the maximum eigenvalue of $S$. The covariances are updated as following:

$$
\begin{aligned}
\bar{P}(k+1 \mid k) & =\lambda_{\max } \mathbf{E}\left[A(Z) A(Z)^{T}\right]-\bar{K}_{p} \bar{R}_{e, k} \bar{K}_{p}^{*} \\
& -\widehat{A} \bar{x}(k \mid k) \bar{x}(k \mid k)^{T} \widehat{A}^{T}+G Q G^{T} \\
\bar{P}(k+1 \mid k+1) & =F \bar{P}(k+1 \mid k) \\
& -F \bar{K}(k+1) H \bar{P}(k+1 \mid k)
\end{aligned}
$$

where $\widehat{A}$ is the expected value of $A(Z)$ and $\bar{K}(k+1)=\left(F \bar{P}(k+1 \mid k) H^{T}+G S\right)(H \bar{P}(k+$ $\left.1 \mid k) H^{T}+R\right)^{-1}$. In the $u b-K F, \mathbf{E}\left[A(Z) A(Z)^{T}\right]$ can be computed in advance but we need to compute $\lambda_{\max }$ at each step of the algorithm. But if the size of $Z$ is large, it is more efficient than the modified KF. (Notice that the computation of $\lambda_{\max }$ requires a polynomial number of operations in $N$ while the size of $\mathcal{Z}$ can be exponential in $N$.)

Lemma 3.3 If $\bar{P}(k \mid k) \geq P(k \mid k)$, then $\bar{P}(k+1 \mid k) \geq P(k+1 \mid k)$. 
Proof. Let $M=\widehat{x}(k \mid k) \widehat{x}(k \mid k)^{T}$ and I be an identity matrix. Then using (3.31), we have

$$
\begin{aligned}
\bar{P}(k \mid k)-P(k \mid k) & =\lambda_{\max } \mathbf{E}\left[A(Z) A(Z)^{T}\right] \\
& -\mathbf{E}\left[A(Z) P(k \mid k) A(Z)^{T}\right]-\mathbf{E}\left[A(Z) M A(Z)^{T}\right] \\
& -K_{p} R_{e, k} K_{p}^{*}+\bar{K}_{p} \bar{R}_{e, k} \bar{K}_{p}^{*} \\
& =\mathbf{E}\left[A(Z)\left(\lambda_{\max }(\bar{P}(k \mid k)) I-P(k \mid k)\right) A(Z)^{T}\right] \\
& +\mathbf{E}\left[A(Z)\left(\lambda_{\max }(M) I-M\right) A(Z)^{T}\right] \\
& -K_{p} R_{e, k} K_{p}^{*}+\bar{K}_{p} \bar{R}_{e, k} \bar{K}_{p}^{*}
\end{aligned}
$$

Since, $\bar{P}(k \mid k) \geq P(k \mid k)$ and $\lambda_{\max }(S) I-S \geq 0$ for any symmetric matrix $S, \bar{P}(k \mid k)-$ $P(k \mid k) \geq 0$.

Using Lemma 3.3, Lemma 3.2, and the induction hypothesis, we obtain the following theorem. The ub-KF maintains the state error covariance which is lower-bounded by the state error covariance of the modified $K F$.

Theorem 3.3 If the $u b-K F$ starts with an initial covariance $\bar{P}(0 \mid 0)$, such that $\bar{P}(0 \mid 0)$ $\geq P(0 \mid 0)$, then $\bar{P}(k \mid k) \geq P(k \mid k)$ for all $k \geq 0$.

\section{Convergence}

The following theorem shows a simple condition under which the state error covariance can be unbounded.

Theorem 3.4 If $\left(\mathbf{E}[A(Z)]^{T}, \mathbf{E}[A(Z)]^{T} C^{T}\right)$ is not stabilizable, or equivalently, $(\mathbf{E}[A(Z)], C \mathbf{E}[A(Z)])$ 
is not detectable, then there exists an initial covariance $P(0 \mid 0)$ such that $P(k \mid k)$ diverges as $k \rightarrow \infty$.

Proof. Let us consider the lb-KF. Let $\underline{P}_{k}=\underline{P}_{k \mid k} . \psi=G Q G^{T}, \hat{A}=\mathbf{E}[A]$, and $F=$ $-\left(C \hat{A} \underline{P}_{k} \hat{A}^{T} C^{T}+C \psi C^{T}+R\right)^{-1}\left(C \psi+C \hat{A} \underline{P}_{k} \hat{A}^{T}\right)$.

Then based on Riccati difference equation [356], we can express $\underline{P}_{k+1}$ as:

$$
\begin{aligned}
\underline{P}_{k+1} & =\hat{A} \underline{P}_{k} \hat{A}^{T}+\psi \\
& -F^{T}\left(C \hat{A} \underline{P}_{k} \hat{A}^{T} C^{T}+C \psi C^{T}+R\right) F \\
& =\left(\hat{A}^{T}+\hat{A}^{T} C^{T} F\right)^{T} \underline{P}_{k}\left(\hat{A}^{T}+\hat{A}^{T} C^{T} F\right) \\
& +F^{T}\left(C \psi C^{T}+R\right) F+\psi C^{T} F+F^{T} C \psi+\psi
\end{aligned}
$$

Hence, if $\left(\hat{A}^{T}+\hat{A}^{T} C^{T} F\right)$ is not a stability matrix, for some $\underline{P}_{0} \leq P(0 \mid 0)$. $\underline{P}_{k}$ diverges as $k \rightarrow \infty$. Since the state error covariance of the lb-KF diverges and $\underline{P}(k \mid k) \leq$ $P(k \mid k)$ for all $k \geq 0$ (Theorem 3.2), $P(k \mid k)$ diverges as $k \rightarrow \infty$. Here $P(k \mid k)$ can be $F_{k} P_{k+1 / k} F_{k}^{*}-K_{k} H_{k} P_{k+1 / k}$ for 'complete' prior case and $K_{k} H_{k} P_{k / k-1}$ for 'without' prior and 'incomplete' prior cases respectively.

\subsection{BAyesian-BAsEd FB Kalman Filter Fusion With- OUT PRIOR INFORMATION}

The Bayesian-Based FB Kalman filter rule of theorem 3.1 is not applicable if either there is no prior information about the estimatee, the information is incomplete (e.g. 
the prior covariance is not known or does not exist), or the estimatee is not random. In these cases, the estimation formulas are not clearly applicable.

The following theorem presents the Bayesian-based FB Kalman filter for without prior information:

\section{Theorem 3.5}

Forward Run: For $(k=0 ; k<T ;+k)$

$$
\begin{aligned}
& \widehat{x}_{k / k}=K_{p, i}\left[y_{i}-\bar{\nu}\right] \\
& \widehat{x}_{k+1 / k}=F_{k} x_{k+1 / k}-K_{p} H_{k} x_{k+1 / k}+k_{p} y-k_{p} \nu \\
& \widehat{P}_{k / k}=K_{k} H_{k} P_{k / k-1} \\
& K_{k}=H_{k}^{+}\left[I-P_{k / k-1}\left(\left(I-H H^{\prime}\right)\left(P_{k / k-1}\right)\right.\right. \\
& \left.\left..\left(I-H H^{\prime}\right)\right)^{+}\right] \\
& \tilde{K}=K+B^{\prime}\left(I-H H^{\prime}\right) \\
& P_{k+1 / k}=K_{p, k} R_{e, k} K_{p, k}^{*}
\end{aligned}
$$

Backward Run: For $(k=0 ; k<T ;+k)$

$$
\begin{aligned}
& \widehat{J}_{k-1 / T}=\widehat{P}_{k-1 / T} F_{k}^{T} \widehat{P}_{k-1 / T}^{-1} \\
& \widehat{x}_{k-1 / T}=\widehat{x}_{k-1 / k-1}^{i}+\widehat{J}_{k-1}\left(\widehat{x}_{k-1 / T}-\widehat{x}_{k-1 / k}\right) \\
& \widehat{P}_{k-1 / T}=\widehat{P}_{k-1 / k-1} \\
& +\widehat{J}_{k-1}\left(\widehat{J}_{k-1 / T}-\widehat{P}_{k-1} / k\right) J_{k-1}^{\prime}
\end{aligned}
$$


where $B$ is any matric of compatible dimensions satisfying $P_{k / k-1}^{\frac{1}{2}^{\prime}}\left(I-H H^{+}\right) B=0$, $P_{k / k-1}^{\frac{1}{2}}$ is any square root matrix of $P_{k / k-1}$. The optimal gain matrix $\tilde{K}$ is given uniquely by:

$$
\begin{aligned}
\tilde{K}= & K=H^{+}\left[I-P_{k / k-1}\left(I-H H^{+}\right)^{\frac{1}{2}}\left(\left(I-H H^{+}\right)^{\frac{1^{\prime}}{2}}\right.\right. \\
& \left.\left.P_{k / k-1}\left(I-H H^{+}\right)^{\frac{1}{2}}\right)^{-1}\left(I-H H^{+}\right)^{\frac{1^{\prime}}{2}}\right]
\end{aligned}
$$

if and only if $\left[H, P_{k / k-1}^{\frac{1}{2}}\right]$ has full row rank, where $\left(I-H H^{+}\right)^{\frac{1}{2}}$ is a full-rank square root of T. Note that in $\hat{x}_{k / k}, \bar{x}_{k}$ is not carried because of no prior information, and all other variables are derived according with condition of $H$ as full row rank.

\subsubsection{MOdifiEd KALMAN FILTER Without PRIOR INFORMATION}

In this section, we outline the case without prior information. As Section 3.5 is discussed for complete prior information, the modification of the kalman filter is focused towards the prediction covariance computing of that case.

Hence, the prediction covariance in the case of no prior information can be computed as following:

$$
\begin{aligned}
P(k+1 \mid k)= & \mathbf{E}\left[e(k+1 \mid k) e(k+1 \mid k)^{T} \mid y_{k}\right] \\
= & -K_{p} R_{e, k} K_{p}^{*} \\
& +\sum_{z \in Z} p_{z} A(z) \widehat{x}(k \mid k) \widehat{x}(k \mid k)^{T}(A(z)-\widehat{A})^{T}
\end{aligned}
$$


And here also, given $\widehat{x}(k+1 \mid k)$ and $P(k+1 \mid k), \widehat{x}(k+1 \mid k+1)$ and $P(k+1 \mid k+1)$ are computed as in the standard Kalman filter.

$$
\begin{aligned}
& \widehat{x}(k+1 \mid k+1)=K(k+1)[y(k+1)-\bar{\nu}] \\
& P(k+1 \mid k+1)=K(k+1) H(k+1) P(k+1)
\end{aligned}
$$

where $K(k+1)=H(k+1)^{+}\left[I-P(k+1)\left(\left(I-H H^{T}\right)(P k+1)\right.\right.$.

\subsubsection{ApProximating the Kalman Filter for Without Prior INFORMATION}

Likewise in Section 3.4.2, since the computation of $P(k+1 \mid k)$ is the only timeconsuming process, we propose two filtering method which can bound $P(k+1 \mid k)$. The same notations have been followed as in Section 3.4.2.

\section{Lower-Bound KF: Without Prior Information Case}

The lower-bound KF (lb-KF) is the same as the modified KF described in Section 3.5.1, except we approximate $P(k+1 \mid k)$ by $\underline{P}(k+1 \mid k)$ and $P(k \mid k)$ by $\underline{P}(k \mid k)$. The covariances are updated as following:

$$
\begin{aligned}
\underline{P}(k+1 \mid k) & =\underline{K}(k+1) \underline{R K}^{T}(k+1) \\
\underline{P}(k+1 \mid k+1) & =\underline{K}(k+1) H \underline{P}^{T}(k+1 \mid k)
\end{aligned}
$$

where $\underline{K}(k+1)=H^{+}\left[I-\underline{P}(k+1 \mid k)\left(\left(I-H H^{T}\right) \underline{P}(k+1 \mid k)\right.\right.$. 
Lemma 3.4 If $\underline{P}(k \mid k) \leq P(k \mid k)$, then $\underline{P}(k+1 \mid k) \leq P(k+1 \mid k)$.

Proof. Using (3.53), we have

$$
\begin{aligned}
P(k+1 \mid k)-\underline{P}(k+1 \mid k) & =\mathbf{E}\left[A(Z) \widehat{x}(k \mid k) \widehat{x}(k \mid k)^{T} A(Z)^{T}\right] \\
& -K_{p, k} R_{e, k} K_{p, k}^{*} \\
& -\widehat{A} \widehat{x}(k \mid k) \widehat{x}(k \mid k)^{T} \widehat{A}^{T} \\
& -\underline{K}_{p, k} \underline{R}_{e, k} \underline{K}_{p, k}^{*} \\
& =P_{1}+P_{2}
\end{aligned}
$$

where $P_{1}=K_{p, k} R_{e, k} K_{p, k}^{*} \quad$ and $\quad P_{2}=\mathbf{E}\left[A(Z) \widehat{x}(k \mid k) \widehat{x}(k \mid k)^{T} A(Z)^{T}\right]-$ $\widehat{A} \widehat{x}(k \mid k) \widehat{x}(k \mid k)^{T} \widehat{A}^{T}-\underline{K}_{p, k} \underline{R}_{e, k} \underline{K}_{p, k}^{*}$.

Since $P(k \mid k)$ is a symmetric matrix, $P(k \mid k)$ can be decomposed into $P(k \mid k)=$ $U_{1} D_{1} U_{1}^{T}$, where $U_{1}$ is a unitary matrix and $D_{1}$ is a diagonal matrix, but there is no $P(k \mid k)$ for $P_{1}$ here. Hence,

$$
P_{1}=-K_{p, k} R_{e, k} K_{p, k}^{*}
$$

\section{Upper-bound KF: Without Prior Information Case}

Similar to the $\mathrm{lb}-\mathrm{KF}$, the upper-bound KF (ub-KF) approximates $P(k+1 \mid k)$ by $\bar{P}(k+$ $1 \mid k)$ and $P(k \mid k)$ by $\bar{P}(k \mid k)$. Let $\lambda_{\max }=\lambda_{\max }(\bar{P}(k \mid k))+\lambda_{\max }\left(\widehat{x}(k \mid k) \widehat{x}(k \mid k)^{T}\right)$, where $\lambda_{\max }(S)$ denotes the maximum eigenvalue of $S$. The covariances are updated as fol- 
lowing:

$$
\begin{aligned}
\bar{P}(k+1 \mid k) & =\lambda_{\max } \mathbf{E}\left[A(Z) A(Z)^{T}\right] \\
& +K_{p, k} R_{e, k} K_{p, k}^{*} \\
\bar{P}(k+1 \mid k+1) & =\bar{K}(k+1) H \bar{P}(k+1 \mid k)
\end{aligned}
$$

where $\bar{K}(k+1)=H^{+}\left[I-\bar{P}(k+1 \mid k)\left(I-H H^{\prime}\right)(\bar{P}(k+1 \mid k)) . \quad\right.$ In the ub-KF, $\mathbf{E}\left[A(Z) A(Z)^{T}\right]$ can be computed in advance but we need to compute $\lambda_{\max }$ at each step of the algorithm.

Lemma 3.5 If $\bar{P}(k \mid k) \geq P(k \mid k)$, then $\bar{P}(k+1 \mid k) \geq P(k+1 \mid k)$.

Proof. Let $M=\widehat{x}(k \mid k) \widehat{x}(k \mid k)^{T}$ and I be an identity matrix. Then using (3.53), we have (See Eqn. (3.62)).

$$
\begin{aligned}
\bar{P}(k \mid k)-P(k \mid k) & =\lambda_{\max } \mathbf{E}\left[A(Z) A(Z)^{T}\right] \\
& -\mathbf{E}\left[A(Z) M A(Z)^{T}\right]-\mathbf{E}\left[\hat{A} M \hat{A}^{T}\right] \\
& =\mathbf{E}\left[A(Z)\left(\lambda_{\max }(M) I-M\right) A(Z)^{T}\right] \\
& +\mathbf{E}\left[\hat{A} M \hat{A}^{T}\right]+\bar{K}_{p, k} \bar{R}_{e, k} \bar{K}_{p, k}^{*} \\
& -K_{p, k} R_{e, k} K_{p, k}^{*}
\end{aligned}
$$

Since, $\bar{P}(k \mid k) \geq P(k \mid k)$ and $\lambda_{\max }(S) I-S \geq 0$ for any symmetric matrix $S, \bar{P}(k \mid k)-$ $P(k \mid k) \succeq 0$

Using Lemma 3.5, Lemma 3.2, and the induction hypothesis, we obtain the follow- 
ing theorem. The ub-KF maintains the state error covariance which is lower-bounded by the state error covariance of the modified $K F$.

Theorem 3.6 If the ub-KF starts with an initial covariance $\bar{P}(0 \mid 0)$, such that $\bar{P}(0 \mid 0)$ $\geq P(0 \mid 0)$, then $\bar{P}(k \mid k) \geq P(k \mid k)$ for all $k \geq 0$.

\section{Convergence}

The convergence will same as followed in Section 3.4.2 and in Theorem 3.4.

\subsection{BAYESIAN-BASED FB KALMAN FILTER FUSION WITH INCOMPLETE PRIOR INFORMATION}

In practice, it is sometimes the case when prior information of some of all the states

of system parameters but not all the components of $\bar{x}$ are not available. For example, tracking the positioning of a vehicle, it is easy to determine the prior position vector of the vehicle (it must be within a certain position range) with certain covariance, but not the velocity of the vehicle, i.e. at what speed it is traveling. Such an incomplete prior problem is presented in this section using Bayesian-based FB Kalman filter.

The following theorem presents the Bayesian-Based FB Kalman filter with incomplete prior information: 


\section{Theorem 3.7}

$$
\begin{aligned}
& \text { Forward Run: For }(k=0 ; k<T ;+k) \\
& \widehat{x}_{k / k}=V K_{p, i} V_{1}^{\prime} \bar{x}+V K_{p, i}\left[y_{i}-\bar{\nu}\right] \\
& \widehat{x}_{k+1 / k}=V K_{p, i} V_{1}^{\prime} \widehat{x}_{k+1 / k}+V K_{p, k} y_{k}-V K_{p, k} V^{\prime} \\
& \widehat{P}_{k / k}=K_{k} H_{k} P_{k / k-1} \\
& K_{k}=H_{k}^{+}\left[I-P_{k / k-1}\left(\left(I-H H^{\prime}\right)\left(P_{k / k-1}\right)\right.\right. \\
& \left.\left..\left(I-H H^{\prime}\right)\right)^{+}\right] \\
& P_{k+1 / k}=G_{i} Q_{i} G_{i}^{*}-K_{p, k} R_{e, k} K_{p, k}^{*}
\end{aligned}
$$

$$
\begin{aligned}
& \text { Backward Run: For }(k=0 ; k<T ;+k) \\
& \widehat{J}_{k-1 / T}=\widehat{P}_{k-1 / T} F_{k}^{T} \widehat{P}_{k-1 / T}^{-1} \\
& \widehat{x}_{k-1 / T}=\widehat{x}_{k-1 / k-1}^{i}+\widehat{J}_{k-1}\left(\widehat{x}_{k-1 / T}-\widehat{x}_{k-1 / k}\right) \\
& \widehat{P}_{k-1 / T}=\widehat{P}_{k-1 / k-1} \\
& +\widehat{J}_{k-1}\left(\widehat{J}_{k-1 / T}-\widehat{P}_{k-1} / k\right) J_{k-1}^{\prime}
\end{aligned}
$$

Proof. By Theorem 3.5, the problem can be converted to without prior information with $H$ and $C$ replaced by the $\tilde{H}$ and $\tilde{C}$ respectively, where, from the proof of Theorem 3.5, the estimatee is $u=V^{\prime} x$, where $V$ is an orthogonal matrix. This means that Theorem 3.5 is applicable now to $u$. Therefore, all formulas in this theorem follows 
from Theorem 3.5 and the relationship:

$$
\widehat{x}=V \hat{u}, P=V \operatorname{MSE}(\hat{u}) V^{\prime}
$$

The uniqueness result thus follows from Theorem 3.5.

\subsubsection{Modified Kalman Filter With Incomplete Prior IN- FORMATION}

In this section, we outline the case with incomplete prior information. As Section 3.6 is discussed for incomplete prior information, the modification of the kalman filter is focused towards the prediction covariance computing of that case.

The prediction covariance in the case of incomplete prior information can be computed as following:

$$
\begin{aligned}
P(k+1 \mid k)= & \mathbf{E}\left[e(k+1 \mid k) e(k+1 \mid k)^{T} \mid y_{k}\right] \\
= & G Q G^{T}-K_{p} R_{e, k} K_{p}^{*} \\
& +\sum_{z \in Z} p_{z} A(z) \widehat{x}(k \mid k) \widehat{x}(k \mid k)^{T}(A(z)-\widehat{A})^{T}
\end{aligned}
$$

And here also, given $\widehat{x}(k+1 \mid k)$ and $P(k+1 \mid k), \widehat{x}(k+1 \mid k+1)$ and $P(k+1 \mid k+1)$ 
are computed as in the standard Kalman filter (See Eqn. (3.72) and (3.72)).

$$
\begin{aligned}
& \widehat{x}(k+1 \mid k+1)=K(k+1)[y(k+1)-\bar{\nu}] \\
& P(k+1 \mid k+1)=K(k+1) H(k+1) P(k+1)
\end{aligned}
$$

where $K(k+1)=\tilde{H}(k+1)^{+}\left[I-\tilde{P}(k+1 \mid k)\left(\left(I-\tilde{H} \tilde{H}^{T}\right)(P k+1 \mid k)\right.\right.$.

\subsubsection{APPROXIMATING THE KALMAN FILTER FOR INCOMPLETE PRIOR \\ INFORMATION}

Likewise in Section 3.4.2, since the computation of $P(k+1 \mid k)$ is the only timeconsuming process, we propose two filtering methods which can bound $P(k+1 \mid k)$. The same notations have been followed as in Section 3.4.2.

\section{Lower-Bound KF: Incomplete Prior Information Case}

The lower-bound KF (lb-KF) is the same as the modified KF described in Section 3.6.1, except we approximate $P(k+1 \mid k)$ by $\underline{P}(k+1 \mid k)$ and $P(k \mid k)$ by $\underline{P}(k \mid k)$. The covariances are updated as following:

$$
\begin{aligned}
\underline{P}(k+1 \mid k) & =G Q G^{T}-\underline{K}_{p, k} \underline{R}_{e, k} \underline{K}_{p, k}^{*} \\
\underline{P}(k+1 \mid k+1) & =V \underline{K}(k+1) H_{k} \underline{P}(k+1 \mid k)^{*} V^{T}
\end{aligned}
$$

where $\underline{K}(k+1)=\tilde{H}_{k}^{+}\left[I-\underline{\tilde{P}}(k+1 \mid k)\left(I-\tilde{H} \tilde{H}^{\prime}\right)(\underline{\tilde{P}}(k+1 \mid k))\right.$.

Lemma 3.6 If $\underline{P}(k \mid k) \preceq P(k \mid k)$, then $\underline{P}(k+1 \mid k) \preceq P(k+1 \mid k)$. 
Proof. Using (3.71), we have

$$
\begin{aligned}
P(k+1 \mid k)-\underline{P}(k+1 \mid k) & =\mathbf{E}\left[A(Z) \widehat{x}(k \mid k) \widehat{x}(k \mid k)^{T} A(Z)^{T}\right] \\
& -K_{p, k} R_{e, k} K_{p, k}^{*} \\
& -\widehat{A} \widehat{x}(k \mid k) \widehat{x}(k \mid k)^{T} \widehat{A}^{T} \\
& +\underline{K}_{p, k} \underline{R}_{e, k} \underline{K}_{p, k}^{*} \\
& =P_{1}+P_{2}
\end{aligned}
$$

where $P_{1}=-K_{p, k} R_{e, k} K_{p, k}^{*} \quad$ and $\quad P_{2}=\mathbf{E}\left[A(Z) \widehat{x}(k \mid k) \widehat{x}(k \mid k)^{T} A(Z)^{T}\right]-$ $\widehat{A} \widehat{x}(k \mid k) \widehat{x}(k \mid k)^{T} \widehat{A}^{T}-\underline{K}_{p, k} \underline{R}_{e, k} \underline{K}_{p, k}^{*}$.

Note: Here we have simply used (3.71), subtract lower bound covariance from the nominal covariance and assign their names as $P_{1}$ and $P_{2}$ respectively. Sections 3.4.1 and 3.4.2 can be seen for more basic details.

Since $P(k \mid k)$ is a symmetric matrix, $P(k \mid k)$ can be decomposed into $P(k \mid k)=$ $U_{1} D_{1} U_{1}^{T}$, where $U_{1}$ is a unitary matrix and $D_{1}$ is a diagonal matrix, but here there is no $P(k \mid k)$ for $P_{1}$.

\section{Upper-bound KF: Incomplete Prior Information Case}

Similar to the lb-KF, the upper-bound KF (ub-KF) approximates $P(k+1 \mid k)$ by $\bar{P}(k+$ $1 \mid k)$ and $P(k \mid k)$ by $\bar{P}(k \mid k)$. Let $\lambda_{\max }=\lambda_{\max }(\bar{P}(k \mid k))+\lambda_{\max }\left(\widehat{x}(k \mid k) \widehat{x}(k \mid k)^{T}\right)$, where $\lambda_{\max }(S)$ denotes the maximum eigenvalue of $S$. The covariances are updated as fol- 
lowing:

$$
\begin{aligned}
\bar{P}(k+1 \mid k) & =\lambda_{\max } \mathbf{E}\left[A(Z) A(Z)^{T}\right] \\
& +\bar{K}_{p, k} \bar{R}_{e, k} \bar{K}_{p, k}^{*} \\
\bar{P}(k+1 \mid k+1) & =\bar{K}(k+1) H \bar{P}(k+1 \mid k)
\end{aligned}
$$

where $\bar{K}(k+1)=\tilde{H}^{+}\left[I-\overline{\tilde{P}}(k+1 \mid k)\left(I-\tilde{H} \tilde{H}^{\prime}\right)(\overline{\tilde{P}}(k+1 \mid k))\right.$. In the ub-KF, $\mathbf{E}\left[A(Z) A(Z)^{T}\right]$ can be computed in advance but we need to compute $\lambda_{\max }$ at each step of the algorithm.

Lemma 3.7 If $\bar{P}(k \mid k) \geq P(k \mid k)$, then $\bar{P}(k+1 \mid k) \geq P(k+1 \mid k)$.

Proof. Let $M=\widehat{x}(k \mid k) \widehat{x}(k \mid k)^{T}$ and I be an identity matrix. Then using (3.71), we have

$$
\begin{aligned}
\bar{P}(k \mid k)-P(k \mid k) & =\mathbf{E}\left[A(Z)\left(\lambda_{\max }(M) I-M\right) A(Z)^{T}\right] \\
& +\hat{A} M \hat{A}^{T}+\bar{K}_{p, k} \bar{R}_{e, k} \bar{K}_{p, k}^{*} \\
& -K_{p, k} R_{e, k} K_{p, k}^{*} \\
& +G Q G^{T}
\end{aligned}
$$

Since, $\bar{P}(k \mid k) \geq P(k \mid k)$ and $\lambda_{\max }(S) I-S \geq 0$ for any symmetric matrix $S, \bar{P}(k \mid k)-$ $P(k \mid k) \geq 0$

Using Lemma 3.7, Lemma 3.2, and the induction hypothesis, we obtain the following theorem. The ub-KF maintains the state error covariance which is lower-bounded by the state error covariance of the modified $K F$. 


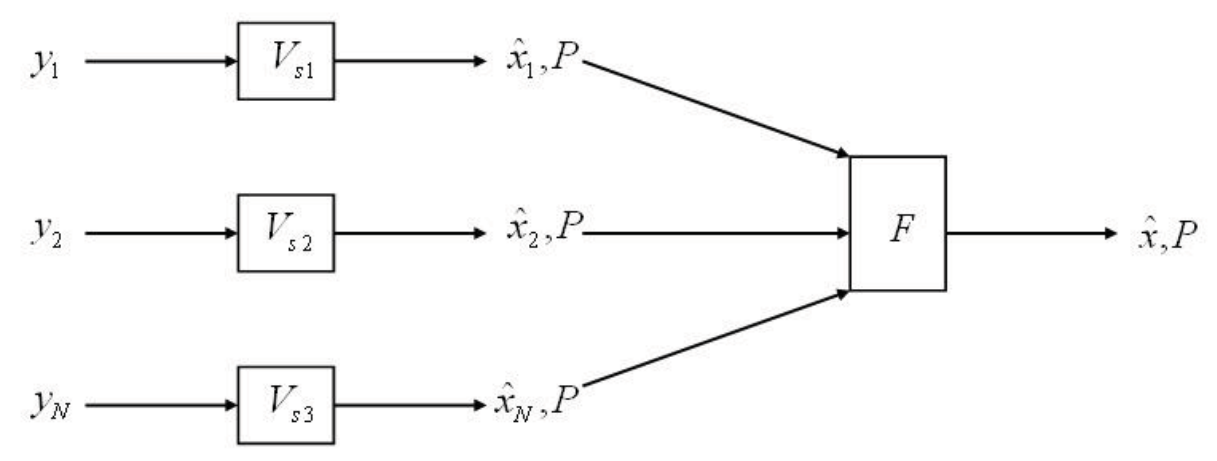

Figure 3.1: Proposed Data Fusion Design

Theorem 3.8 If the ub-KF starts with an initial covariance $\bar{P}(0 \mid 0)$, such that $\bar{P}(0 \mid 0)$ $\geq P(0 \mid 0)$, then $\bar{P}(k \mid k) \geq P(k \mid k)$ for all $k \geq 0$.

\section{Convergence}

The convergence will be the same as followed in Section 3.4.2 and in Theorem 3.4.

\subsection{Fusion ALgORITHM}

The information captured in each priori cases are designed for a distributed structure. The idea is taken from [357].

Suppose there is $X$ number of sensors. For every measurement coming from these sensors that is received in fusion center, there is a corresponding estimation based solely on one sensors that taken is so called virtual sensor (VS). Every estimation from Single VS then is processed through the fusion algorithm to get optimal estimation of the state. Overall diagram of fusion process using multiple sensors can be seen in Fig. 3.1. 
When estimate of the states are available, based on their prior knowledge, now the problem turn how to combine these different estimations to get the optimal result. Fused estimation based on the series of particular sensors are computed every sampling time $T_{s}$, where the fused estimation $\hat{x}(k \mid k)$ is no more than an estimation coming from each sensor $\hat{x}_{i}(k \mid k)$ (See Theorem 3.9 where equation no. 3.81-3.84 comprise the fusion algorithm).

Theorem 3.9 For any $k=1,2, \ldots$, the estimate and the estimation error covariance of $x(k)$ based on all the observations before time $k T$ are denoted by $\hat{x}(k \mid k)$ and $P(k \mid k)$, then they can be generated by use of the following formula:

$$
\begin{aligned}
& \hat{x}(k \mid k)=\sum_{i=1}^{N} \alpha_{i}(k) \hat{x}_{N \mid i}(k \mid k) \\
& P(k \mid k)=\left(\sum_{i=1}^{N} P_{N \mid i}^{-1}(k \mid k)\right)^{-1}
\end{aligned}
$$

where,

$$
\alpha_{i}(k)=P(k \mid k) P_{N \mid i}^{-1}(k \mid k)
$$

where $\hat{x}_{N \mid i}(k \mid k)$ is state estimation at the highest sample rate based on estimation from $V S i$ and $P_{N \mid i}(k \mid k)$ is it's error covariance.

From equation (3.82), it can be verified that:

$$
P(k \mid k) \leq P_{N \mid i}(k \mid k)
$$


which means that the fused estimation error from estimation of different sensors are always be less or equal to the estimation error of each sensor.

\subsection{Evaluation AND Testing}

The evaluation and testing has been made on an electro-hydraulic system [358]. A networked control system with wired communication has been developed in a Matlab environment as can be seen in the Fig. 3.2. In simulation, we study the performance of the modified Kalman filtering algorithms developed for various types of prior information against the standard Bayesian-Based Kalman smoother which assumes no communication errors. Then we provide motivating results showing the effectiveness of the $\mathrm{lb}-\mathrm{KF}$ and ub-KF. Our simulation is based on a Matlab environment developed for multiple fault scenarios in a wired networked control system. For each test case, we will run the modified Bayesian-Based KF and the standard Bayesian-KF and show their comparisons for various cases, moreover compute the mean square error (MSE) of state estimates and show the results in Table I, II, and III respectively.

Fault scenarios are created by using the rotational hydraulic drive in the simulation program. In these scenarios leakage fault and controller fault are being considered.

Scenario I: Leakage Fault In this scenario, while the system is working in real time, leakage faults is being introduced in the hydraulic fluid flow linked to the servo-valve of the system. The leakage fault is considered as $\omega_{h} C_{L_{\text {leakage }}} x_{3}(t)$ in state 3 . 


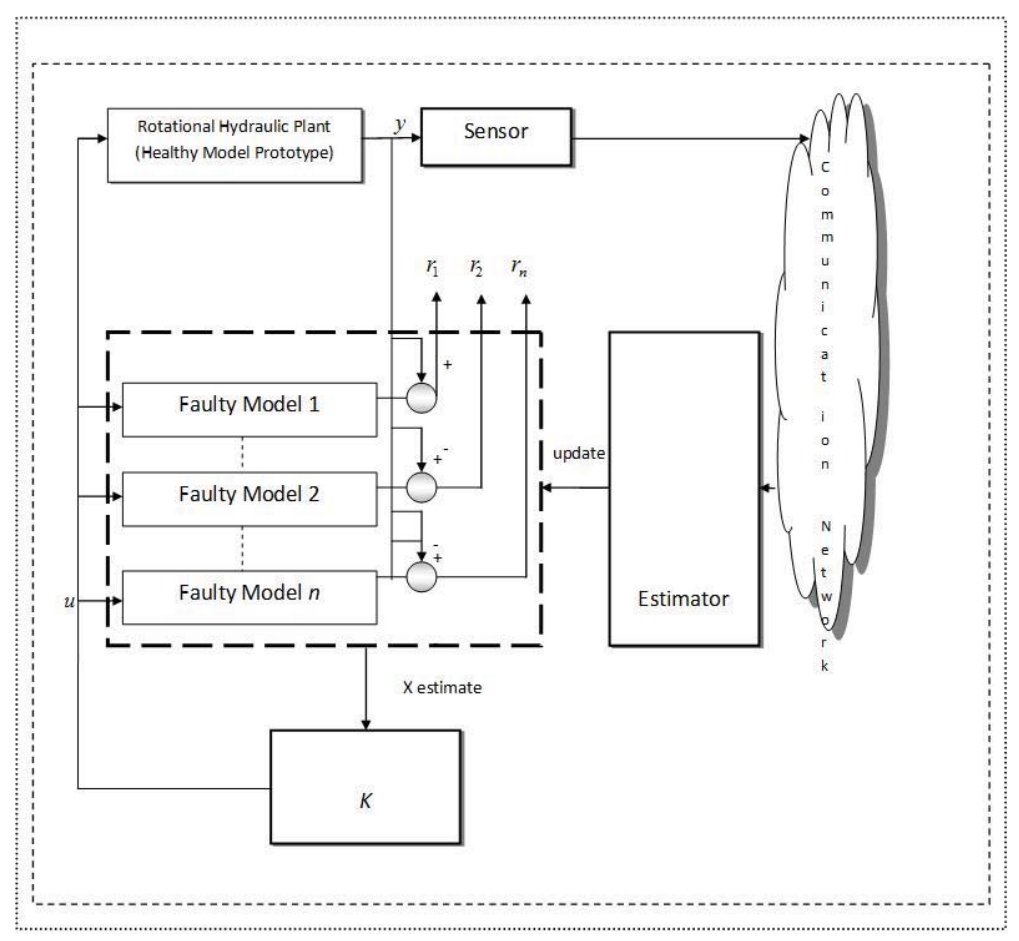

Figure 3.2: Architecture of LTIP in distributed control network 
Scenario II: Controller Fault In this scenario, while the system is working in real time and getting the input for driving the dynamics of the system, a fault has been introduced by increasing the torque load in the hydraulic drive, then effecting the controller, $-\frac{\omega_{h}}{\alpha} t_{L_{\text {fault }}}$ is considered in state 2 of the system. Following [358] and the fault scenarios, the fault model of the system can be described in state space form as:

$$
\begin{aligned}
\dot{x}_{1}(t)= & \omega_{\max } x_{2}(t) \\
\dot{x}_{2}(t)= & -\gamma \frac{\omega_{h}}{\alpha} x_{2}(t)+\frac{\omega_{h}}{\alpha} x_{3}(t)- \\
& \frac{\omega_{h}}{\alpha} t_{L}-\frac{\omega_{h}}{\alpha} t_{L_{\text {fault }}} \\
\dot{x}_{3}(t)= & -\alpha \omega_{h} x_{2}(t)-\omega_{h} C_{L} x_{3}(t) \\
+ & \alpha \omega_{h} x_{4}(t) \sqrt{1-x_{3}(t) \operatorname{sigm}\left(x_{4}(t)\right)} \\
- & \omega_{h} C_{L_{\text {leakage }}} x_{3}(t) \\
\dot{x}_{4}(t)= & -\frac{1}{\tau_{v}} x_{4}(t)+\frac{i(t)}{\tau_{v}}
\end{aligned}
$$


where

$$
\begin{aligned}
x_{1}(t) & =\theta(t), x_{2}(t)=\frac{\dot{\theta}(t)}{\omega_{\max }}, \\
x_{3}(t) & =\frac{P_{L}(t)}{P_{s}}, x_{4}(t)=\frac{A_{v}(t)}{A_{v}^{\max }}, \\
u_{1}(t) & =i(t)=\frac{I(t)}{I_{\max }}, u_{2}=t_{L}=\frac{T_{L}}{P_{s} D_{m}}, \\
\gamma & =\frac{B \omega_{\max }}{P_{s} D_{m}}, \\
\omega_{h} & =\sqrt{\frac{2 \beta D_{m}^{2}}{J V}}, \\
\alpha & =\frac{\left(C_{d} A_{v}^{\text {max }} \sqrt{P_{s} \rho}\right) J \omega_{h}}{P_{s} D_{m}^{2}}, \\
c_{L} & =\frac{J C_{L} \omega_{h}}{D_{m}^{2}}
\end{aligned}
$$

and $C_{L_{\text {leakage }}}$ is the leakage fault considered in state $3, t_{L_{\text {fault }}}$ is the controller fault in the form of torque load in state 2 .

Using the sign convention for $A_{v}(t)$ and the definition of $x_{3}(t)$, it follows that $0 \leq$ $x_{3}(t) \operatorname{sigm}\left(x_{4}(t)\right) \leq 1$. It is also noted here that $0 \leq x_{3}(t) \operatorname{sigm}\left(x_{4}(t)\right) \leq 1$, because $P_{1}(t)$ and $P_{2}(t)$ are both positive and the condition $x_{3}(t) \operatorname{sigm}\left(x_{4}(t)\right)=1$ implies that $P_{1}(t)=P_{s}$ and $P_{2}(t)=0$ or $P_{2}(t)=P_{s}$ and $P_{1}(t)=0$, indicating zero pressure drop across the open ports of the servo-valve and thus, no flow to or from the actuator, a situation that would occur if the rotational motion of the drive is impeded.

\subsubsection{EVALUATION OF RESUlts}

In what follows, we present simulation results for the proposed distributed approximate estimation with three cases of prior knowledge. The experiment has been performed on 
the rotational hydraulic drive system. Two sets of faults have been considered here, that is, the leakage fault in state 3 and controller fault. Firstly, the data collected from the plant has been initialized and the parameters have been being optimized which comprises of the pre-processing and normalization of the data. The comparison of results for the distributed estimation, and estimation generated from various levels of faults, and the basic profile of that particular fault has been compared. Moreover, same pattern of comparison has been followed for modified estimation filters with lower bound and upper bound. Later, computational time comparison has been shown for different results showing the effectiveness of the modified filter in all cases.

\section{Fault 1 (Leakage): Estimates and covariance comparison of distributed estimation} with complete prior information, with lower and upper bound filter versions

The Bayesian-Based FB Kalman filter has been simulated here for the leakage fault of the plant. Simulations have been made for the x-estimate and the covariance of each case. In the simulation, comparison of various levels of leakage, that is, no, small, and medium intensity of leakage faults, and distributed estimation has been shown. It can be seen for the estimate profile in Fig. 3.4 that the distributed structure is clearly performing well as compared to the other profiles for complete prior information, when it comes to the covariance of modified filter implementation with upper bound, see Fig. 3.6 and lower bound, see Fig. 3.9 for estimate of lower bound scheme, it is performing equally well for distributed structure. Actually, the advantage of using the modified upper and lower bound filters can be seen more clearly when we talk about the time computation as discussed in the next Section. 
Fault 1 (Leakage): Estimates and covariance comparison of distributed estimation with incomplete prior information, with its lower and upper bound filter versions

In case of incomplete prior information with leakage fault, when it comes to the covariance and estimate of modified filter implementation with upper bound, see Fig. 3.12 it performs well for distributed structure. Actually, the advantage of using the modified upper and lower bound filters can be seen more clearly when we talk about the time computation as discussed in the next Section.

\section{Fault 1 (Leakage): Estimates and covariance comparison of distributed estimation without prior information, with its lower and upper bound filter versions}

In case of estimation without prior information but with leakage fault, it can be seen for the covariance profile, see Fig. 3.13, that the distributed structure is clearly performing well as compared to the other profiles for without prior information, which is the worst scenario case chosen among all three as far as the prior information is concerned. It is only because of the full rank of the $H$ matrix in the gain $K_{k}$ that it managed to show the performance, in particular with distributed case, likewise, when it comes to the covariance and estimate of modified filter implementation with upper bound, see Fig. (3.16 for covariance of upper bound scheme and lower bound, see Fig. 3.18 for covariance of lower bound scheme, it appears to be performing equally well for distributed structure. It is due to this factor of poor prior information that rise in the y-axis (estimate) can be seen. A comparison of computation time will be reported later. 
Fault 2 (Controller): Estimates and Covariance Comparison of Distributed Estimation with Complete Prior Information, with its lower and upper bound filter versions

The Bayesian-Based FB Kalman filter has been simulated here for the controller fault of the plant, which has been introduced by increasing the torque load in the hydraulic drive, then effecting the controller. Simulations have been made for the x-estimate and the covariance of each case. In the simulation, comparison of various levels of controller faults, that is, no, small, and medium intensity of faults, and distributed estimation has been shown. It can be seen for the covariance profile, see Fig. 3.3 and estimate, see Fig. 3.5 that the distributed structure is clearly performing well as compared to the other profiles for complete prior information, when it comes to the covariance and estimate of modified filter implementation with upper bound, see Fig. 3.7 for covariance of upper bound scheme and lower bound, see Fig. 3.8 for covariance of lower bound scheme and see Fig. 3.10 for estimate of lower bound scheme, it is performing equally well for distributed structure.

Fault 2 (Controller): Estimates and Covariance Comparison of Distributed Estimation with Incomplete Prior Information, with its lower and upper bound filter versions

In case of incomplete prior information with controller fault, it can be seen for the estimate profile, see Fig. 3.11 that the distributed structure is clearly performing well as compared to the other profiles even for incomplete prior information. A comparison 
of computation time will be reported later.

\section{Fault 2 (Controller): Estimates and covariance comparison of distributed estima- tion without prior information, with its lower and upper bound filter versions}

In case of estimation without prior knowledge but with controller fault, it can be seen for the covariance profile, see Fig. 3.14 and estimate, see Fig. 3.15 that the distributed structure is clearly performing well as compared to the other profiles for without prior information, which is the worst scenario case chosen among all three as far as the prior information is concerned. It is only because of the full rank of the $H$ matrix in the gain $K_{k}$ that it managed to show the performance, in particular with distributed case, likewise, when it comes to the covariance and estimate of modified filter implementation with upper bound, see Fig. 3.19 for covariance of lower bound scheme and see Fig. 3.21 for estimate of lower bound scheme, it is performing equally well for distributed structure. The advantage of using the modified upper and lower bound filters can be seen more clearly when we talk about the time computation as discussed in the next Section.

\subsubsection{Time Computation}

The time computation of different methods is evaluated using an HP COMPAQ labtop, $\mathrm{n} \times 7300$ INTEL $(\mathrm{R})$ core $(\mathrm{TM}) 2 \mathrm{CPU}$ T $7200 @ 2 \mathrm{GHz}$ with $2.5 \mathrm{~GB}$ ram and 500 Hard disk. An equal number of 5 iterations have been run for achieving each and every of the estimate. For the case of complete prior information, it can be seen from Table 3.1, that iteration time of the basic bayesian-based FB Kalman filter, though it 


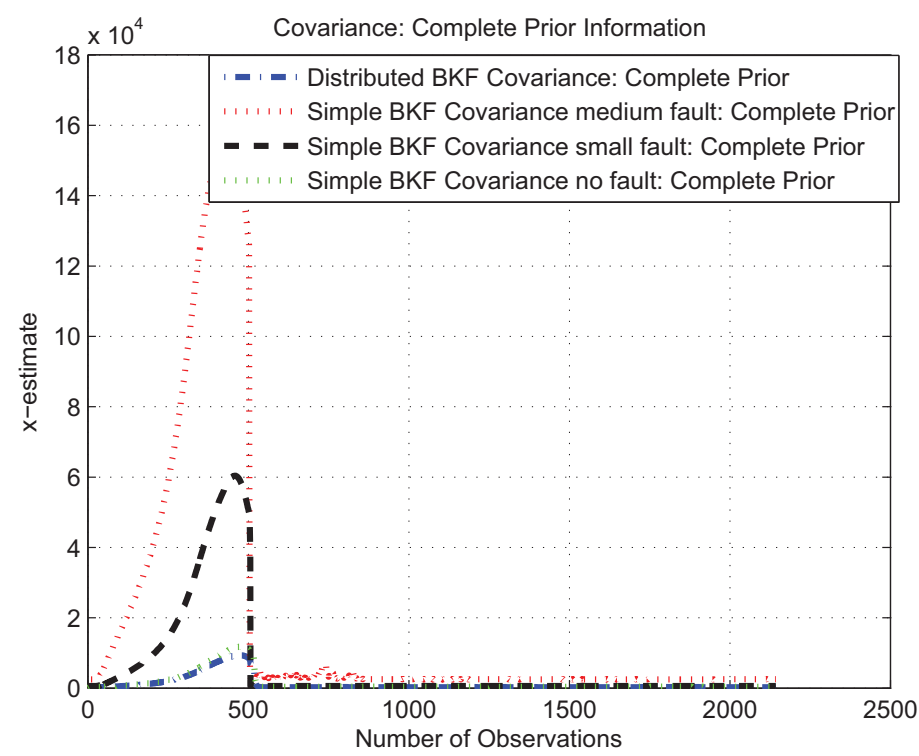

Figure 3.3: Comparison of Covariance for complete prior information for Controller Fault

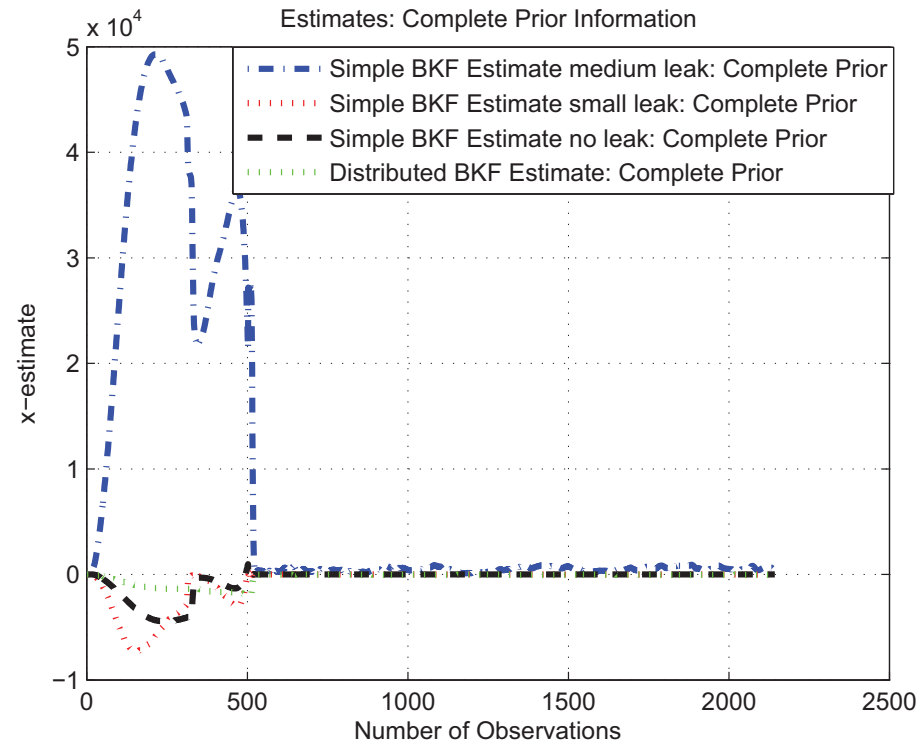

Figure 3.4: Comparison of Estimates for complete prior information for Leakage Fault 


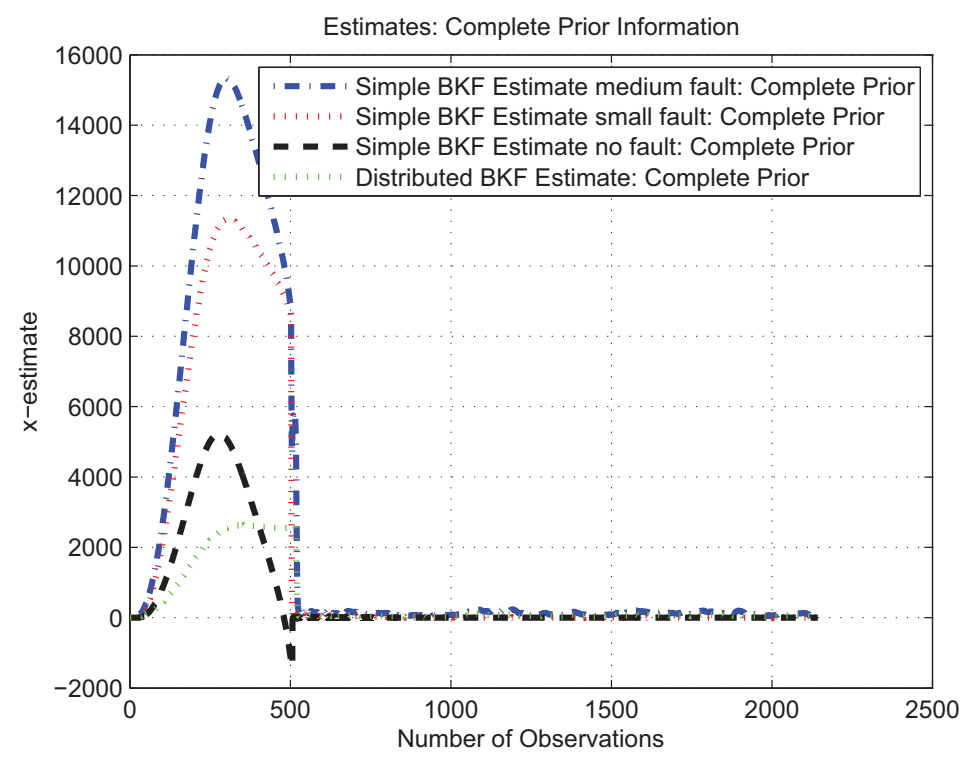

Figure 3.5: Comparison of Estimates for complete prior information for Controller Fault

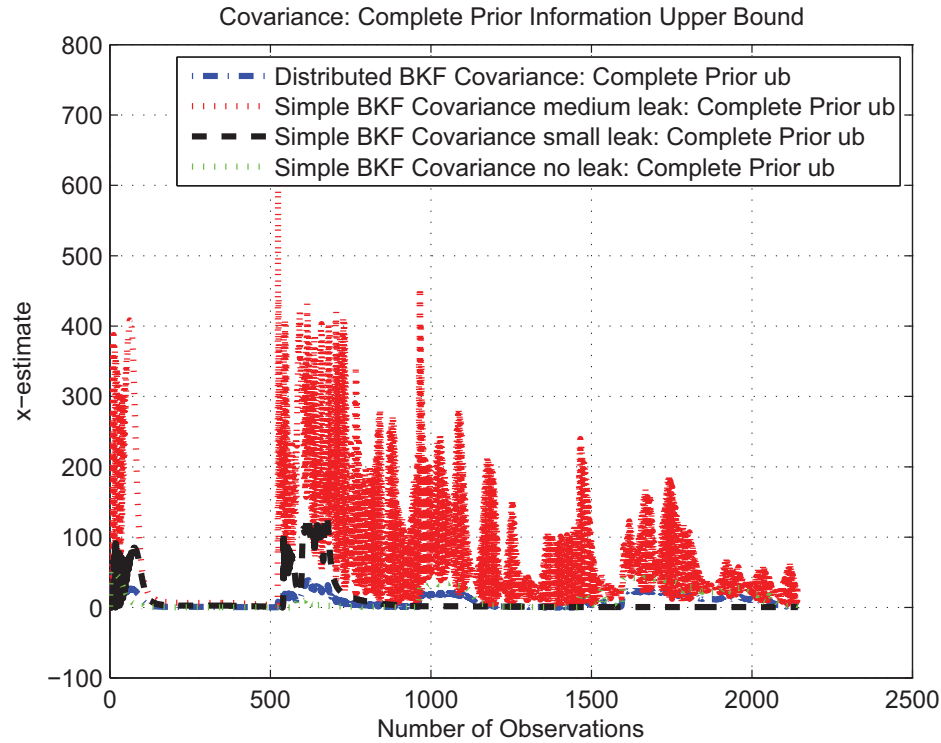

Figure 3.6: Comparison of Covariance for complete prior information for Leakage Fault with Upper Bound Modified Filter 


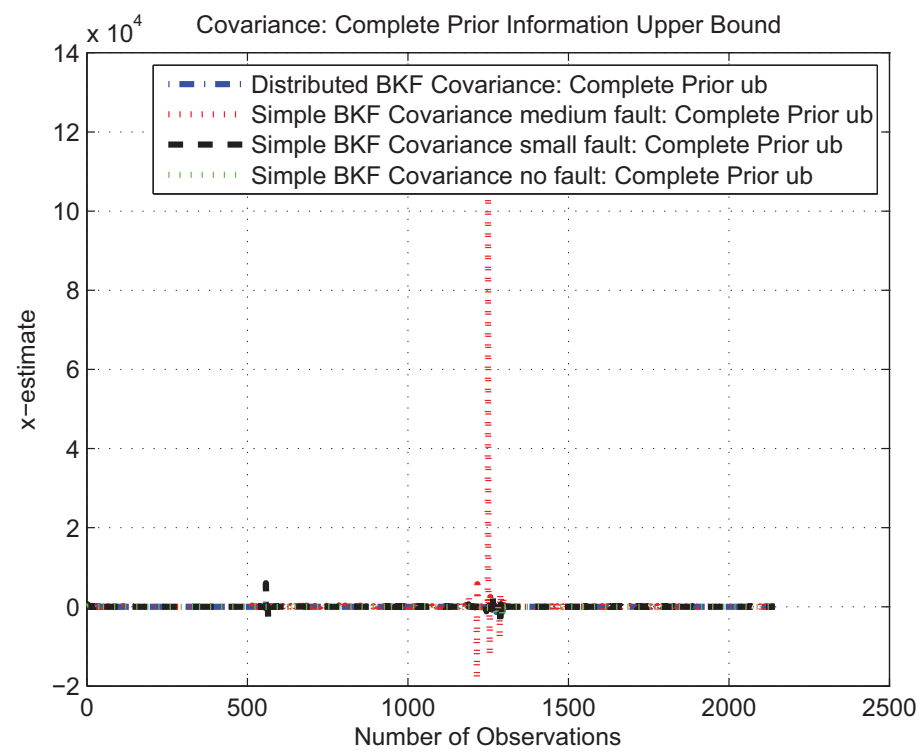

Figure 3.7: Comparison of Covariance for complete prior information for Controller Fault with Upper Bound Modified Filter

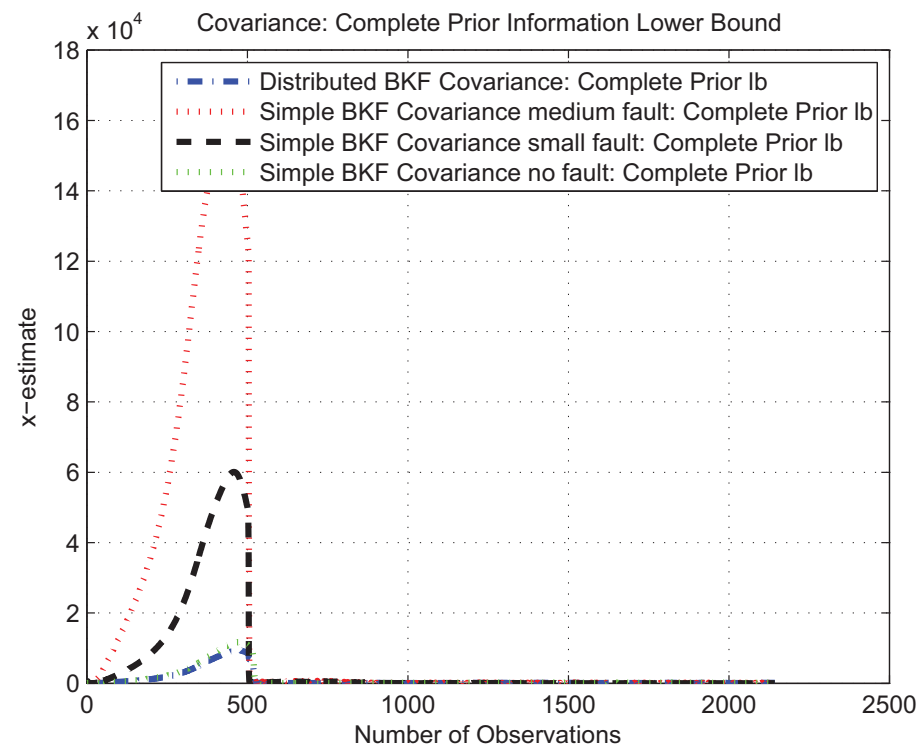

Figure 3.8: Comparison of Covariance for complete prior information for Controller Fault with Lower Bound Modified Filter 


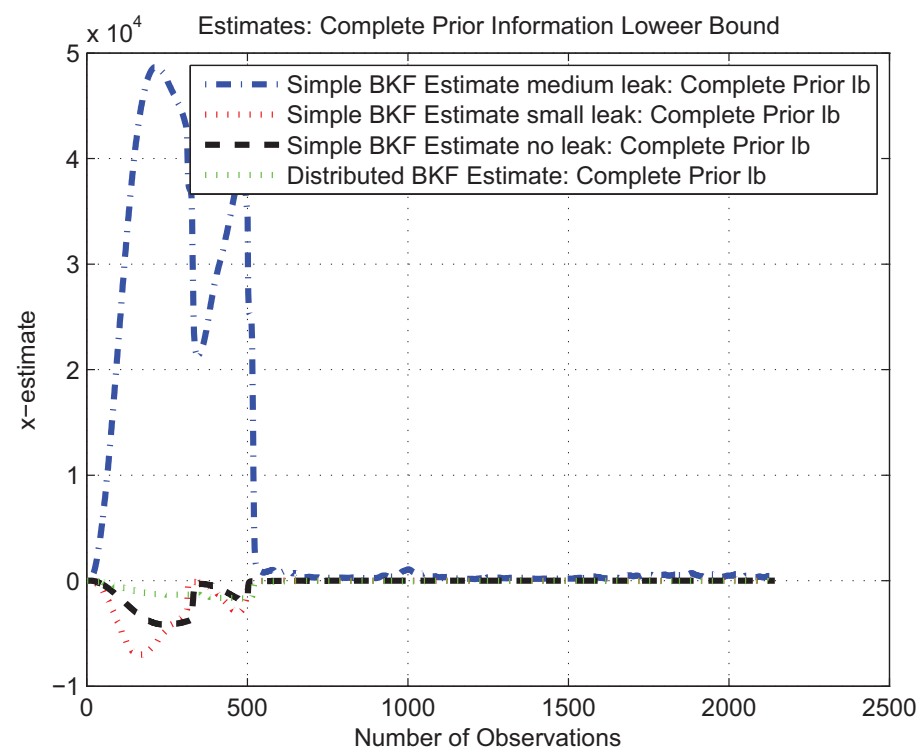

Figure 3.9: Comparison of Estimates for complete prior information for Leakage Fault with Lower Bound Modified Filter

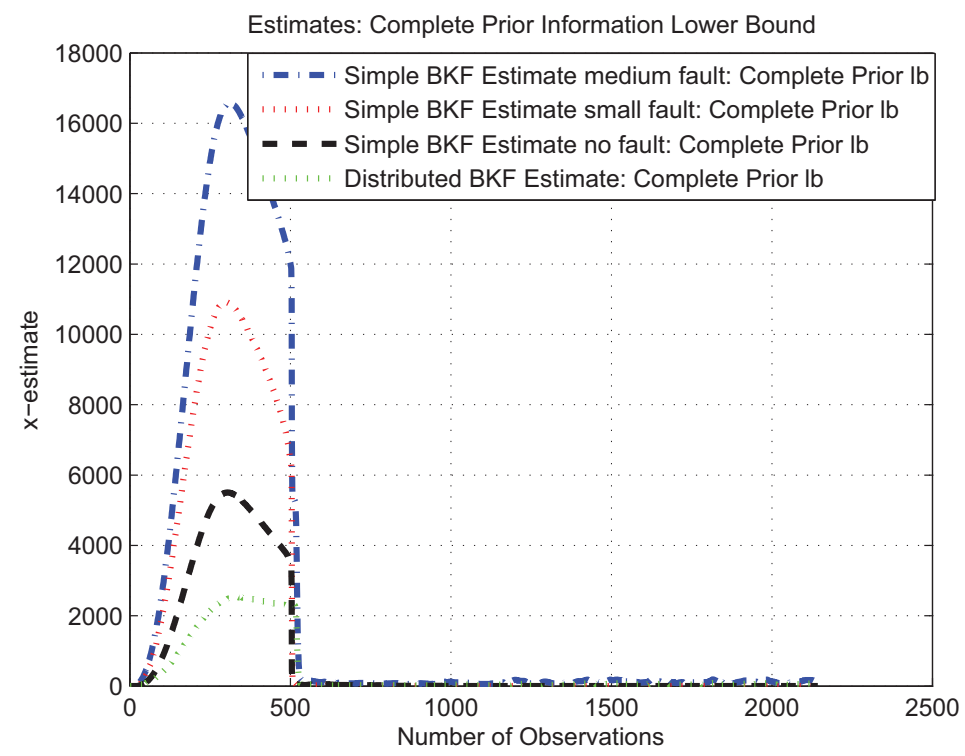

Figure 3.10: Comparison of Estimates for complete prior information for Controller Fault with Lower Bound Modified Filter 


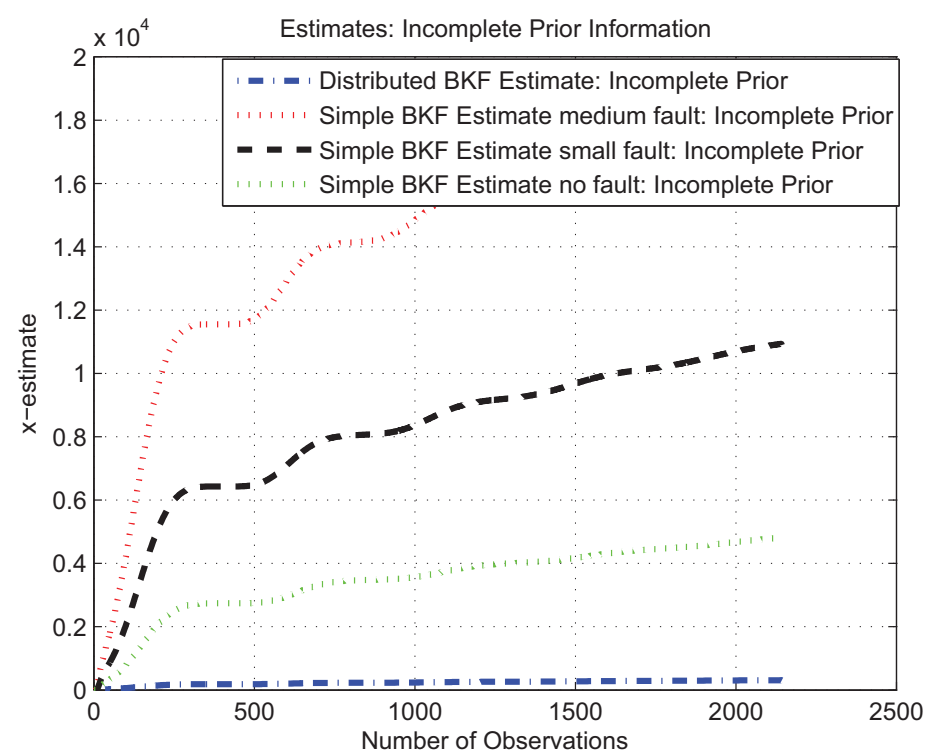

Figure 3.11: Comparison of Estimates for Incomplete prior information for Controller Fault

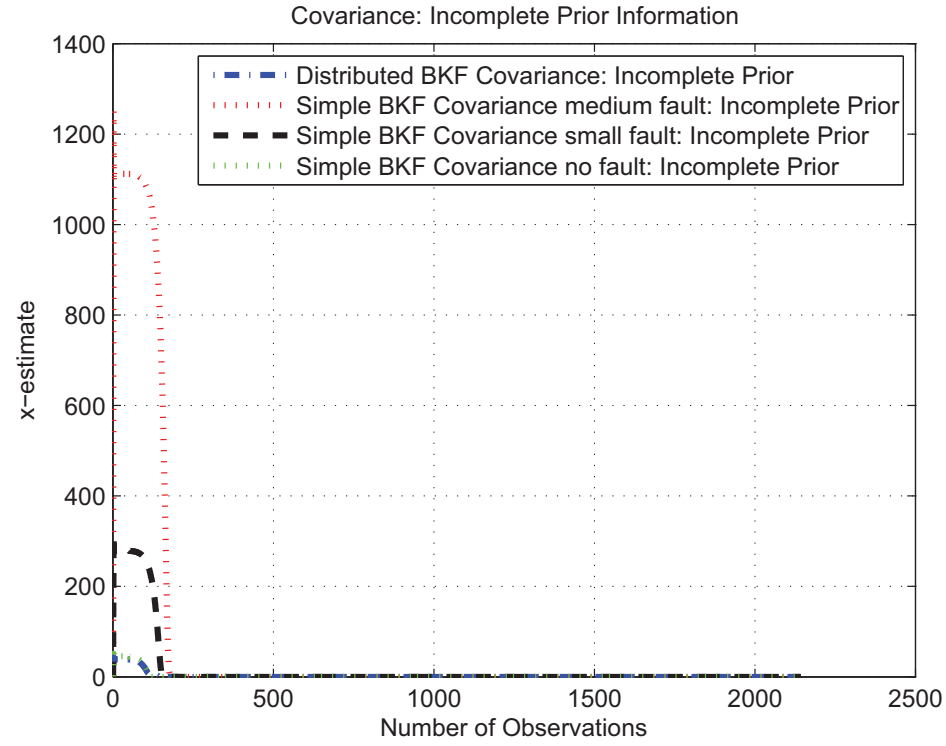

Figure 3.12: Comparison of Covariance for Incomplete prior information for Leakage Fault with Upper Bound Modified Filter 


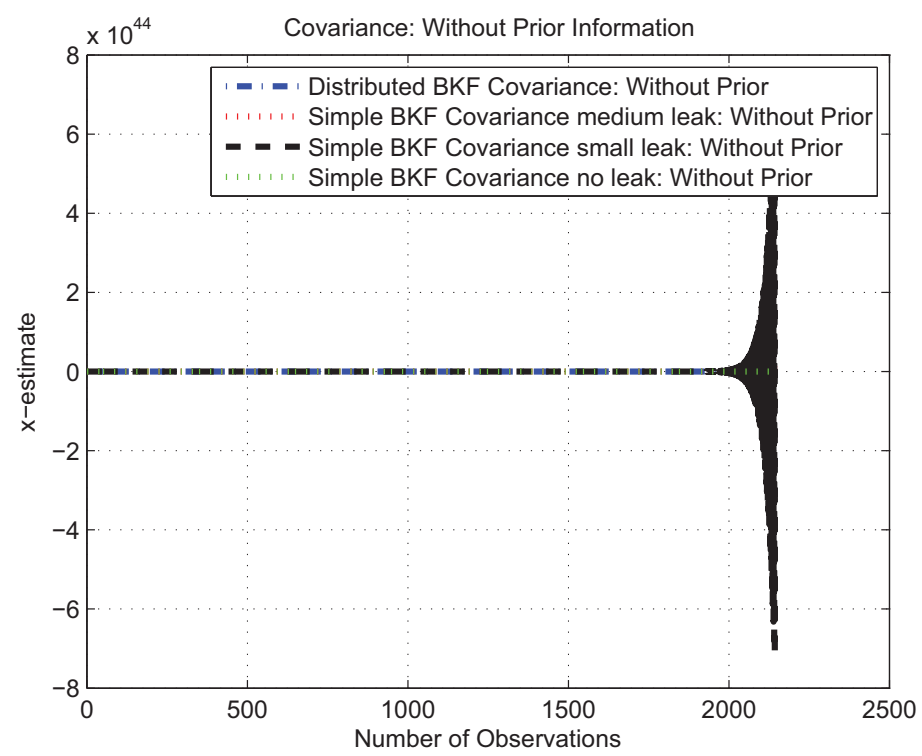

Figure 3.13: Comparison of Covariance for without prior information for Leakage Fault

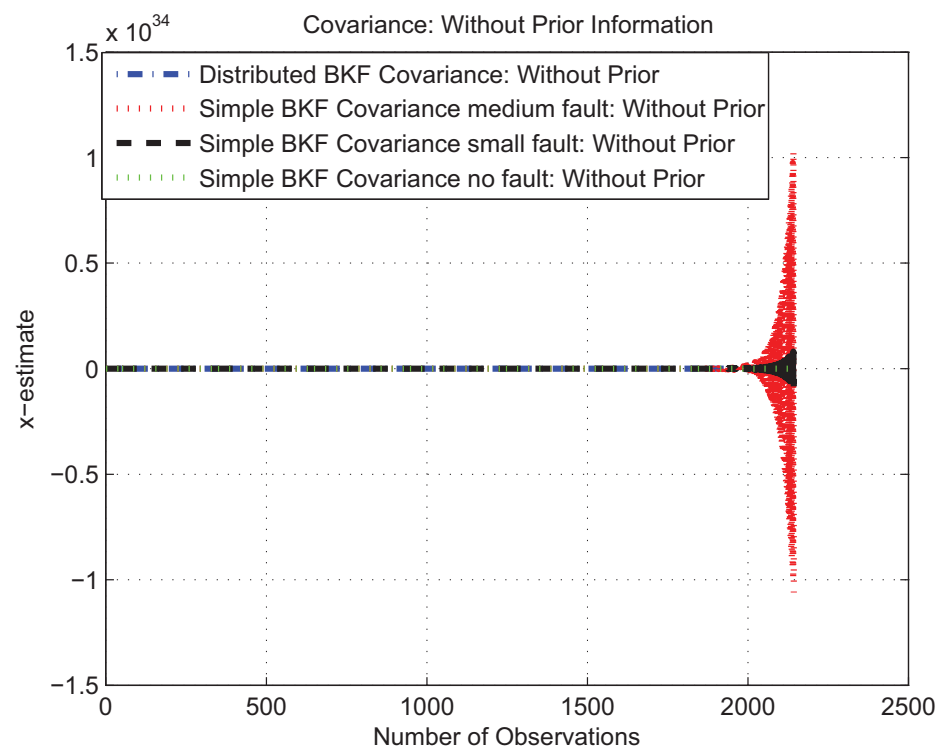

Figure 3.14: Comparison of Covariance for without prior information for Controller Fault 


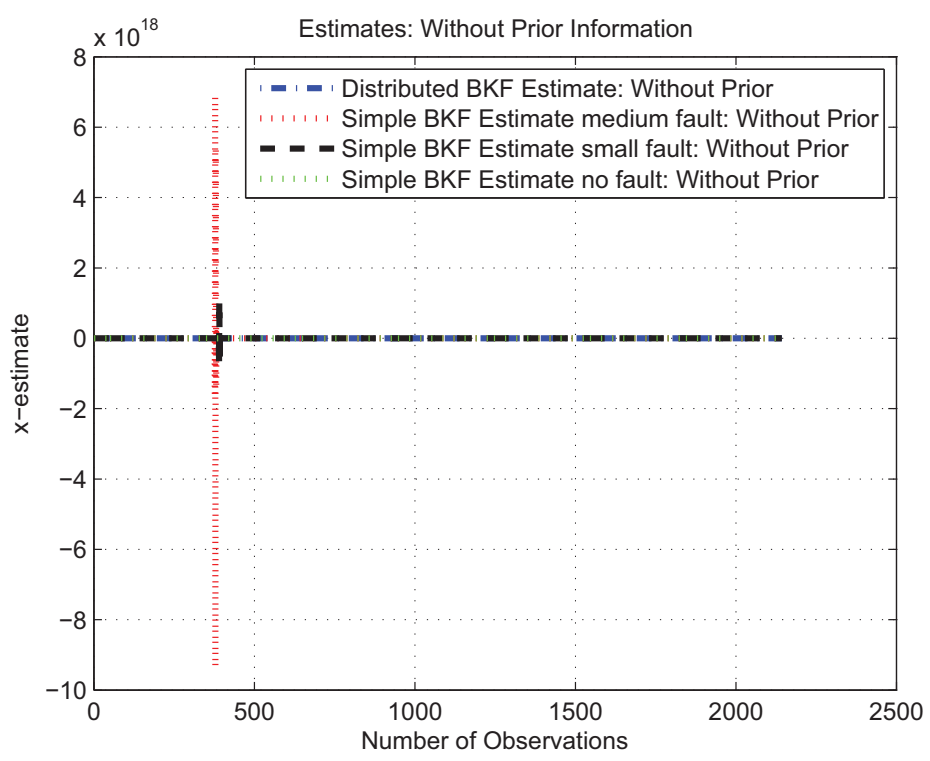

Figure 3.15: Comparison of Estimates for without prior information for Controller Fault

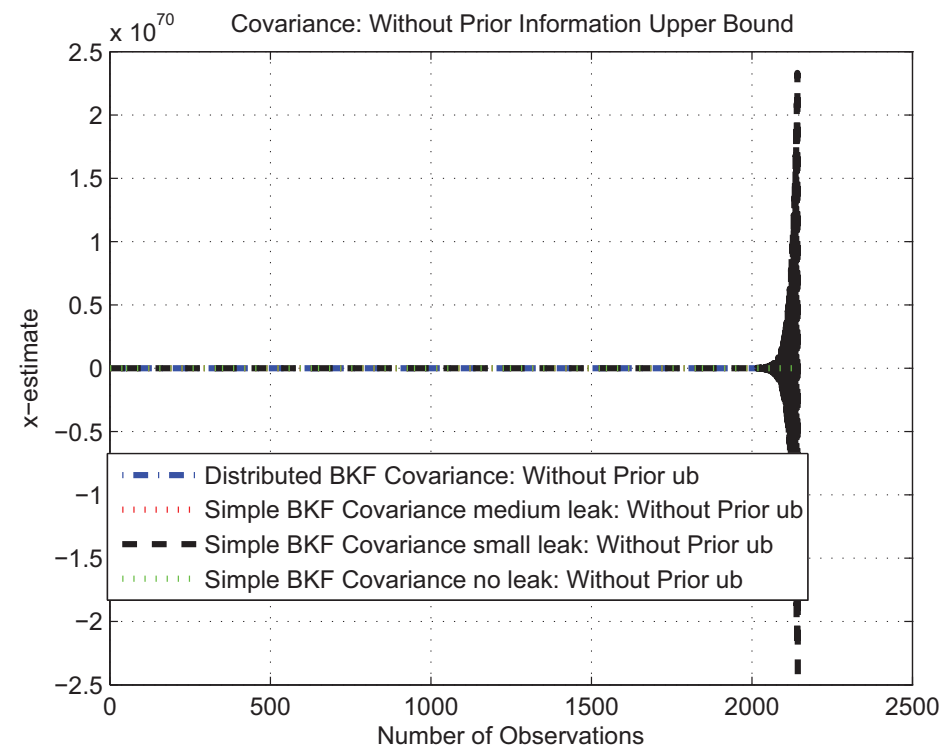

Figure 3.16: Comparison of Covariance for without prior information for Leakage Fault with Upper Bound Modified Filter 


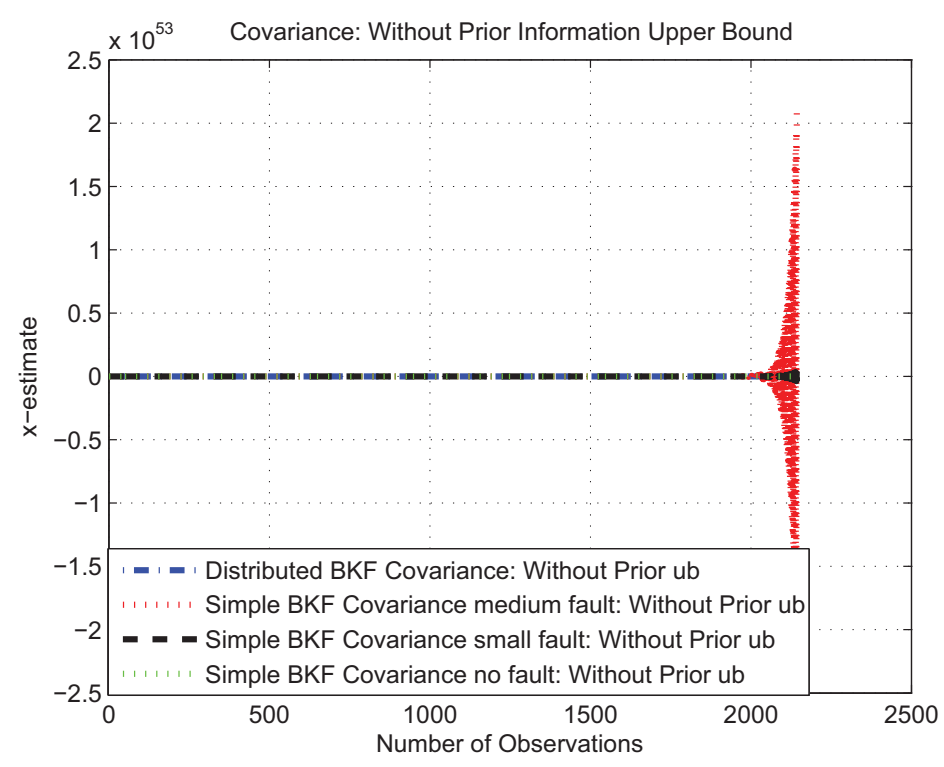

Figure 3.17: Comparison of Covariance for without prior information for Controller Fault with Upper Bound Modified Filter

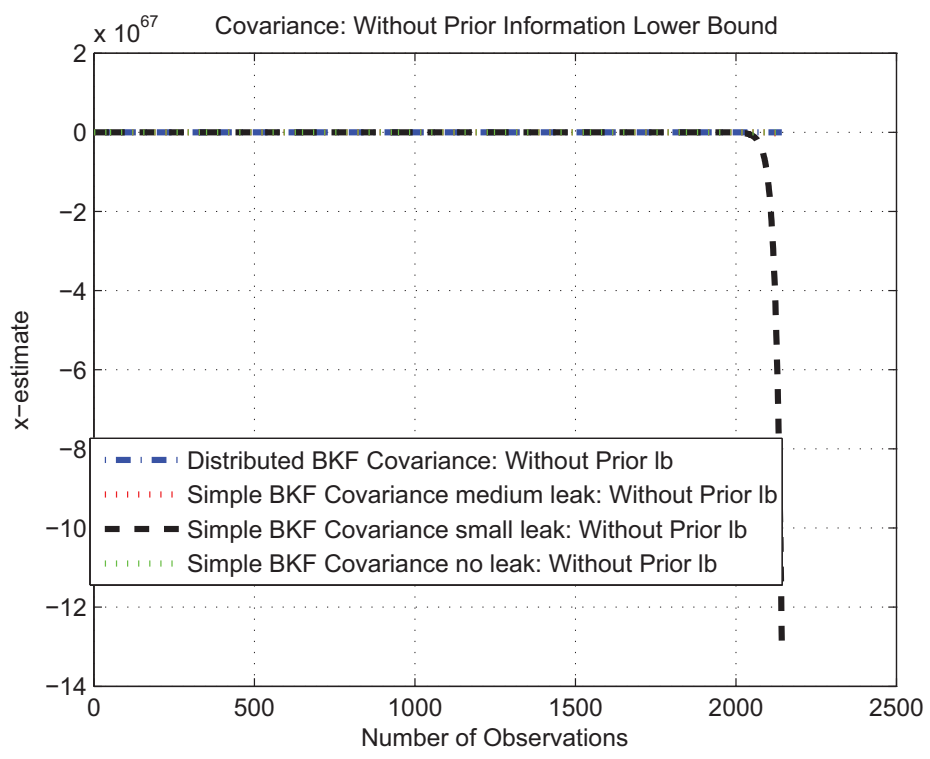

Figure 3.18: Comparison of Covariance for without prior information for Leakage Fault with Lower Bound Modified Filter 


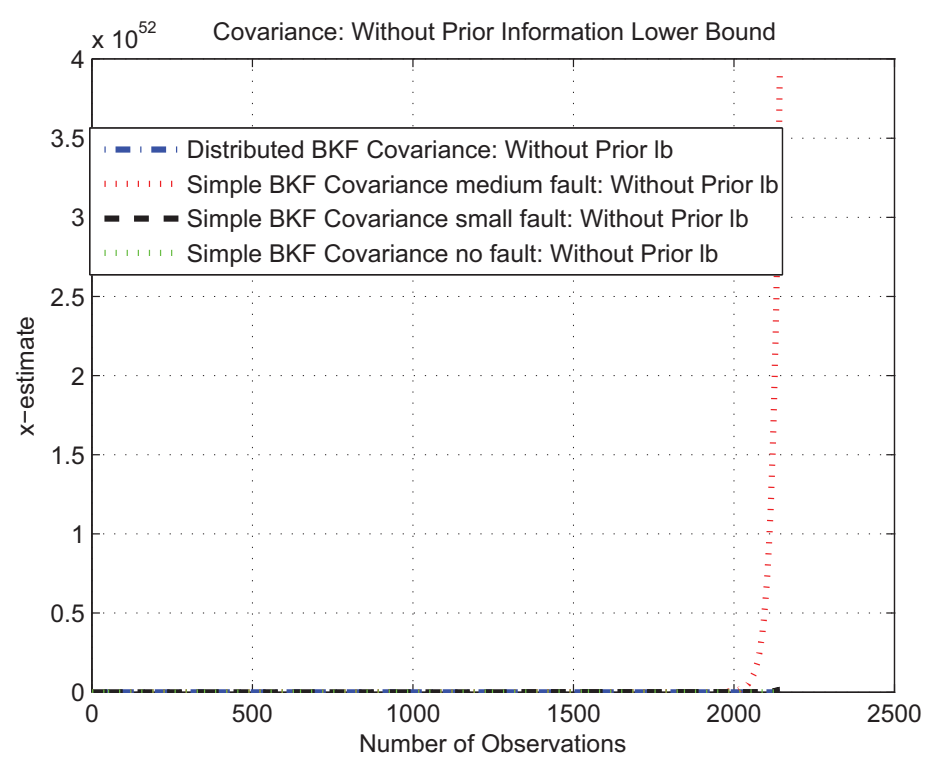

Figure 3.19: Comparison of Covariance for without prior information for Controller Fault with Lower Bound Modified Filter

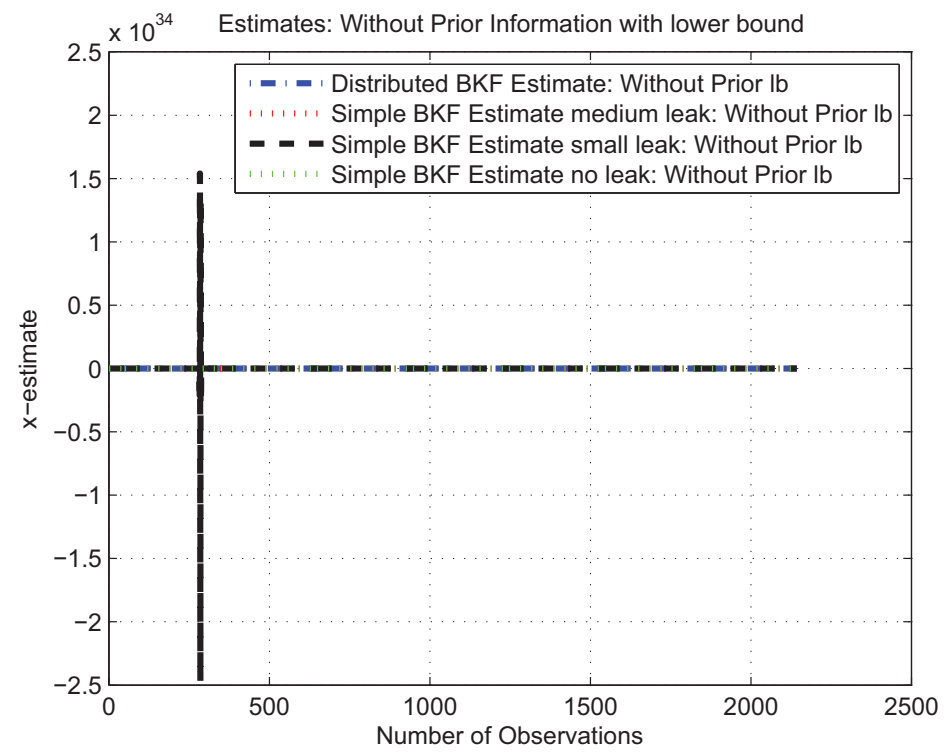

Figure 3.20: Comparison of Estimates for without prior information for Leakage Fault with Lower Bound Modified Filter 


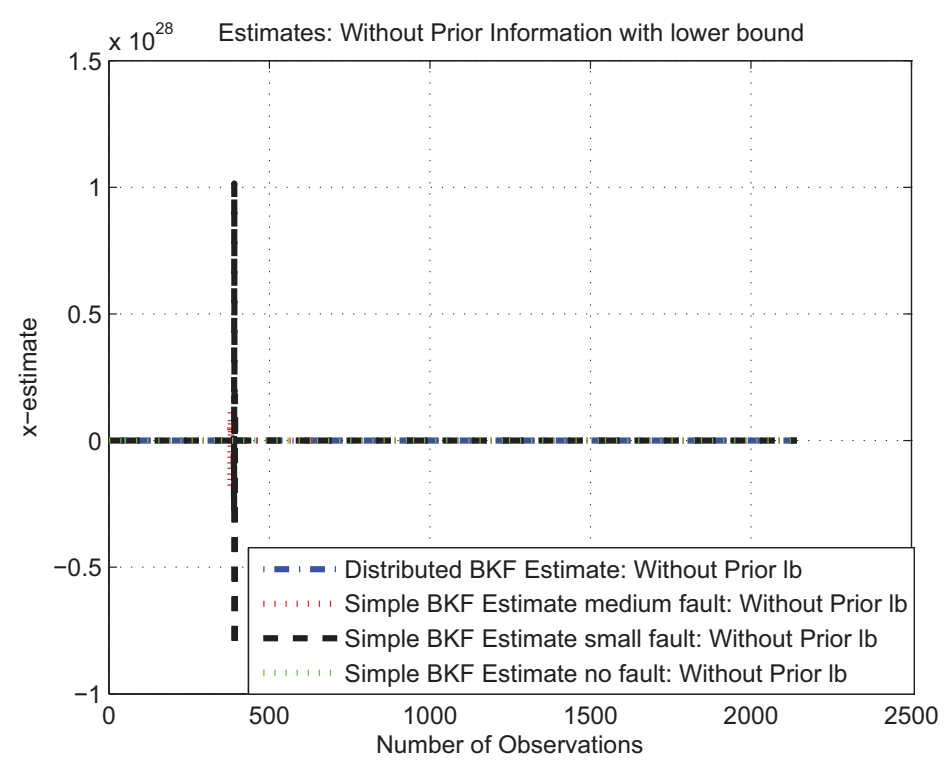

Figure 3.21: Comparison of Estimates for without prior information for Controller Fault with Lower Bound Modified Filter

is very much optimal in nature due to its structure than the regular Kalman filter, is taking the maximum number of time for the computation, whereas both modified filters of upper bound and lower bound are performing well with less computation time for leakage fault (fault 1) and controller fault (fault 2) respectively. Likewise are the cases of incomplete prior information, see Table 3.2 and without prior information in Table 3.3 which are even more crucial and critical because of their structures, and here the basic Bayesian-based FB Kalman filter is taking comparatively more time than the likes of modified lower bound and upper bound filters. The performance of the modified filters was consistent even here for both leakage fault (fault 1) and controller fault (fault 2) respectively. In the tables, Bayesian FB KF+ means with upper bound and Bayesian FB KF- corresponds to with lower bound 
Table 3.1: Case I: Time Computation Comparison for Complete Prior Information

\begin{tabular}{c|c|c|}
\hline Filter & LEAKAGE FAUlt & CONTROLler FAUlt \\
\hline \hline 1- BAYESIAN FB KF & 14.81 & 12.53 \\
\hline 2- BAYESIAN FB KF+ & 12.23 & 12.22 \\
\hline 3- BAYESIAN FB KF- & 12.09 & 12.26 \\
\hline
\end{tabular}

Table 3.2: Case II: Time Computation Comparison for Incomplete Prior Information

\begin{tabular}{c|c|c|}
\hline FiLTER & LEAKAGE FAULT & CONTROLLER FAULT \\
\hline \hline 1- BAYESIAN FB KF & 13.503922 & 12.492827 \\
\hline 2- BAYESIAN FB KF+ & 12.732579 & 12.191222 \\
\hline 3- BAYESIAN FB KF- & 12.939255 & 12.166062 \\
\hline
\end{tabular}

Table 3.3: Case III: Time Computation Comparison for Without Prior Information

\begin{tabular}{c|c|c|}
\hline Filter & LEAKAGE FAult & CONTROLler FAult \\
\hline \hline 1- BAYESIAN FB KF & 23.463690 & 22.445465 \\
\hline 2- BAYESIAN FB KF+ & 22.926070 & 12.165139 \\
\hline 3- BAYESIAN FB KF- & 22.366596 & 21.970777 \\
\hline
\end{tabular}




\section{A Distributed EM-Based Kalman}

\section{SMOOTHER}

\subsection{AN OVERVIEW}

In this chapter, we have discussed Distributed Expectation Maximization(EM)-Based Kalman smoother, where distributed EM-based smoother estimation is derived for both cases of full and reduced-order respectively.

\subsection{INTRODUCTION}

Estimation in distributed structures of different types often provide complementary and overlapping coverage on targets. Estimation using filters and their application in different fields is a wide area of research with intense science. Considering the papers of estimation using filters, [360] presents a procedure for design and tuning of reduced orders $H_{\infty}$ feed-forward compensators for active vibration control systems subject to wide band disturbances. The procedure took in account the inherent positive feedback coupling between the compensator system and the measurement of the image for 
disturbance. In [361], an open-loop observer is designed that estimates each wheel's orientation of the wheel chair based only on the rear wheels' kinematics. The model has been validated by propelling the wheelchair on three different floors (vinyl, carpet, and concrete) with five different normal forces between the caster wheels and the ground. In [362], estimator is implemented on a reduced order version i.e. a linear Kalman filter based on a reduced order electrochemical model is designed to estimate internal battery potentials, concentration gradients, and state-of-charge from external current and voltage measurements. A non-linear version of the estimator is used in [363], where an extended Kalman filter observer is presented to estimate manipulator states and couple these estimates to an adaptive rigid-link flexible-joint controller. When it comes to application of estimators, fault detection and isolation is one of the main areas. In [364], investigation is made on the leakage fault diagnosis problem for a physical internet-based three-tank system. In [365], the problem of designing and developing a hybrid fault detection and isolation scheme for a network of unmanned vehicles is dealt, subject to large environmental disturbances.

In essence, the driving force of estimation in dynamical estimation methods is Kalman filter [366]. Optimal linear smoothers stemming from the estimation theory can be considered as an extension of the Kalman filter, because they take future observations into account. Actually, all optimal linear smoother algorithms involve the Kalman smoother, perhaps because of its property of depending on the $a$-prior knowledge. A detailed description of the various types of smoothers based on Kalman's theory, and their algorithms can be found in [367] and [368]. For the sequential approach of the 
smoothing, as in [369] and [370], the Kalman filter analysis follows a retrospective approach, that is done by making corrections of the past state estimates using the Kalman filter innovation. In this paper, we have derived distributed EM-based smoother estimation for both cases of full and reduced-order respectively. It is done with the help of Kalman-like particle filter. The estimation is derived on a stochastic singular system [371]. After achieving a full and reduced-order distributed structure, we have stemmed EM algorithm in each case. The proposed scheme is then validated on a power quality system implemented in an experimental laboratory, where different types of loads were introduced, and then different load profile data were considered for the evaluation of the proposed scheme.

The main contribution of this paper is Kalman-like particle smoother which has been derived and implemented as full-order and reduced-order respectively with EM algorithm tunes for model paramters. The proposed smoothers have been implemented on a data from power quality system with various comparison of results for three types of loads.

The remainder of this paper is structured as follows. Problem formulation is described in Section II. The distributed full-order EM-based smoother is derived and discussed in Section III, followed by the distributed reduced-order EM-based smoother, derived and discussed in Section IV. Evaluation and testing is made in Section V. Finally some conclusion is described in Section VI. 


\subsection{Problem Formulation}

Consider the discrete-time stochastic singular linear system, as in [371] with multiple sensors given by:

$$
\begin{aligned}
M x_{k+1} & =\Phi x_{k}+\Gamma \omega_{k} \\
y_{k}^{(i)} & =H^{(i)} x_{k}+\nu_{k}^{(i)}, i=1,2, \ldots, l
\end{aligned}
$$

where the state $x_{k} \in \Re^{n}$, the measurements $y_{k}^{(i)} \in \Re^{m_{(i)}}, i=1,2, \ldots, l, \omega_{k} \in \Re^{r}$ and $\nu_{k}^{(i)} \in \Re^{m_{(i)}}, i=1,2, \ldots, l$ are independent white noises with zero mean and variance $Q_{\omega}$ and $Q_{\nu^{(i)}} . M, \Phi, \Gamma, H^{(i)}$ are the constant matrices with compatible dimensions, $l$ is the number of sensors, and the superscript $(i)$ denotes the $i$ th sensor.

Assumption 1. $M$ is a singular square matrix, $\operatorname{rank} M=n_{1}<n, \operatorname{rank} \Phi \geq n_{2}$ and $n_{1}+n_{2}=n$.

Assumption 2. System (4.1) is regular, i.e., $\operatorname{det}(z M-\Phi) \neq 0$ where $z$ is an arbitrary complex.

Assumption 3. The initial state $x(0)$ with mean $\mu_{0}$ and variance $P_{0}$ is independent of $w(t)$ and $v^{(i)}(t), i=1,2, l$.

Our aim is to find the distributed reduced-order fusion Kalman smoother $\hat{x}^{(0)}(t \mid t)$ of the state $x(t)$ based on measurements $\left(y^{(i)}(t), y^{(i)}(1)\right), i=1,2,, l$. 
For system (4.1) and (4.2), there are nonsingular matrices $L$ and $R^{[15]}$, such that:

$$
\begin{gathered}
L M R=\left[\begin{array}{ll}
M_{1} & 0 \\
M_{2} & 0
\end{array}\right], L \Phi R=\left[\begin{array}{cc}
\Phi_{1} & 0 \\
\Phi_{2} & \Phi_{3}
\end{array}\right], \\
L \Gamma R=\left[\begin{array}{c}
\Gamma_{1} \\
\Gamma_{2}
\end{array}\right], H^{(i)} R=\left[\begin{array}{ll}
H_{1}^{(i)} & H_{2}^{(i)}
\end{array}\right]
\end{gathered}
$$

where $M_{1} \in \Re^{n_{1} \times n_{2}}$ is non-singular lower-triangular, $\Phi_{1} \in \Re^{n_{1} \times n_{1}}$ is quasi-lowertriangular, $\Phi_{3} \in \Re^{n_{2} \times n_{2}}$ is non-singular lower-triangular. By introducing the transformation $x(t)=R\left[x_{1}^{T}(t) x_{2}^{T}(t)\right]^{T}$ with $x_{1}(t) \in \Re^{n_{1}}$ and $x_{2}(t) \in \Re^{n_{2}}$, where $T$ denotes the transpose.

The singular system (4.1) and (4.2) is transferred into the following two reducedorder subsystems:

$$
\begin{gathered}
\left\{\begin{array}{c}
x_{1(k+1)}=\Phi_{0} x_{1}(t)+\Gamma_{0} \omega(t) \\
y_{k}^{(i)}=\bar{H}^{(i)} x_{1(k)}+\eta_{k}^{(i)}
\end{array}\right. \\
x_{2(k)}=B x_{1(k)}+C \omega_{k}
\end{gathered}
$$

where $\Phi_{0}=M_{1}^{-1} \Phi_{1}, \Gamma_{0}=M_{1}^{-1} \Gamma_{1}, \bar{H}^{(i)}=H_{1}^{(i)}+H_{2}^{(i)} B, \eta_{k}^{i}=\Gamma_{3}^{(i)} \omega_{k}+\nu_{k}^{(i)}$, $\Gamma_{3}^{(i)}=H_{2}^{(i)} C, B=\Phi_{3}^{-1} M_{2} M_{1}^{-1} \Phi_{1}-\Phi_{3}^{-1} \Phi_{2}, C=\Phi_{3}^{-1} M_{2} M_{1}^{-1} \Gamma_{1}-\Phi_{3}^{-1} \Gamma_{2}$. 
Also, we have

$$
\begin{aligned}
& \mathrm{E}\left\{\left[\begin{array}{c}
\omega(t) \\
\eta^{(i)}(t)
\end{array}\right],\left[\begin{array}{cc}
\omega^{T}(k) & \eta^{(j)^{T}}(k)
\end{array}\right]\right\}=Q^{(i j)} \delta_{t k}, \\
& Q^{(i j)}=\left[\begin{array}{cc}
Q_{\omega} & S^{(j)} \\
S^{(i)^{T}} & Q_{\eta^{(i, j)}}
\end{array}\right],
\end{aligned}
$$

where $S^{(i)}=Q_{\omega} \Gamma_{3}^{(i)^{T}}, Q_{\eta^{(i i)}}=Q_{\eta^{(i)}}=\Gamma_{3}^{(i)} Q_{\omega} \Gamma_{3}^{(i)^{T}}+Q_{\nu^{(i)}}$ and $Q_{\eta^{(i j)}}=\Gamma_{3}^{(i)} Q_{\omega} \Gamma_{3}^{(i)^{T}}$, $i \neq j$. E is the expectation, and $\delta_{t k}$ is the Kronecker delta function.

\subsection{Full-ORdER EM-BASEd Fusion SMOOTHERS}

In this Section, and the Section coming ahead i.e. Section 4.5, we will derive Kalmanlike particle smoother fusion with full-order and reduced order respectively. The Kalmanlike particle smoother is expressed as follows with $i$ th sensor (See Equation (4.7-4.13)), where the simple Kalman-like particle filter is expressed in [414]. A question arises here that why Kalman-like particle smoother has been preferred on a basic Kalman smoother? The justification for the approach w.r.t filter is given in [414], moreover, it is preferred here as a smoother on the basic kalman smoother because of the following. (See Fig. 4.1 for the comparison of estimates of a basic kalman smoother and Kalmanlike particle smoother. See Fig. 4.2 where it can be seen, how the mean square error is reduced in less number of iterations for particle smoother as compared to a regular Kalman smoother): 


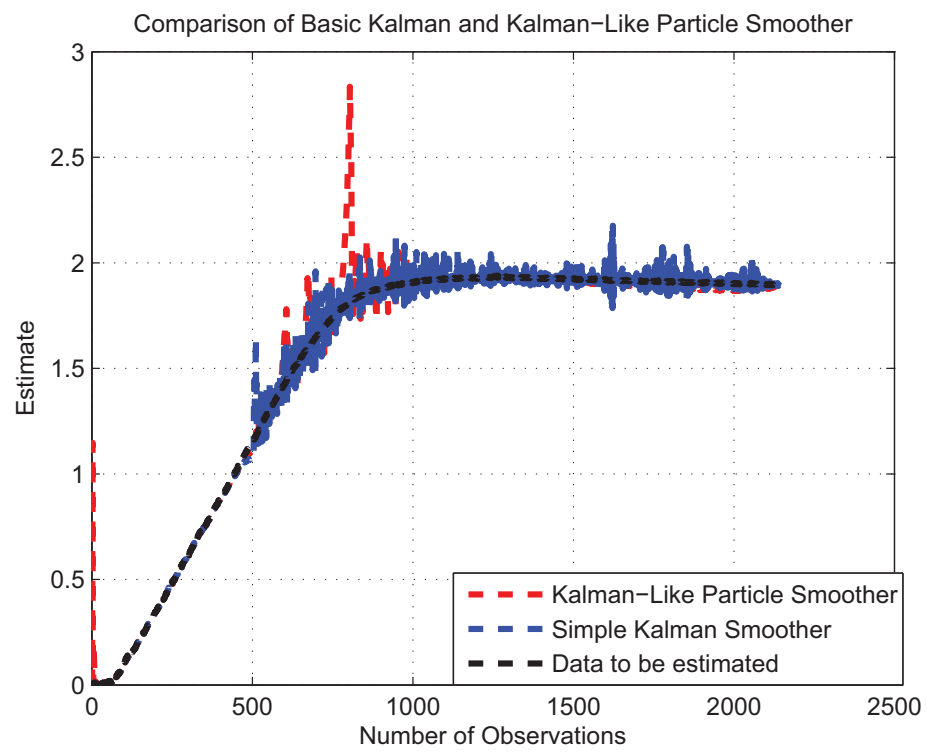

Figure 4.1: Estimates of Kalman-like particle and Basic Kalman smoother

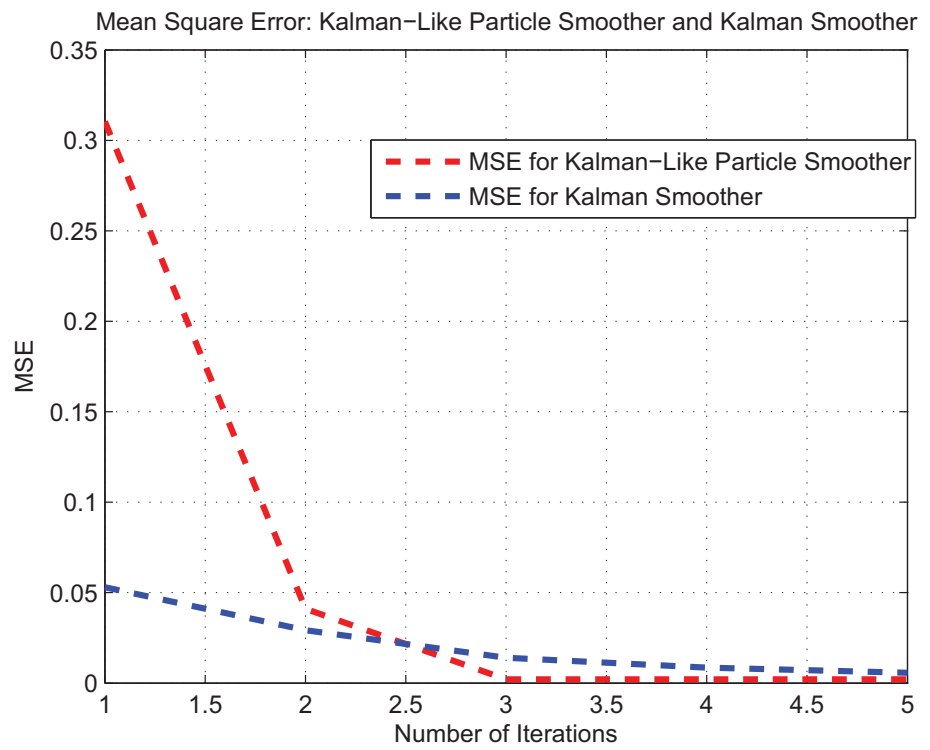

Figure 4.2: Mean Square Error: Kalman-like particle and basic Kalman smoother 
Initialization: $x_{0}^{a}$ and $P_{0}$

Forecast Step:

$$
\begin{aligned}
& x_{k / k-1}^{f_{i}}=\Phi_{k-1, k} x_{k-1 / k-1}^{a_{i}}, I \in \Sigma_{k} \\
& P_{k, i / k-1}^{f_{i} a_{i}}=\Phi_{k-1 / k} P_{k-1, i \mid k-1}^{a_{i} a_{i}}, i \in \Sigma_{k}, \\
& P_{k / k-1}^{f_{i}}=\Phi_{k-1 / k}\left(P_{k, k-1 \mid k-1}^{f_{i} a_{i}}\right)^{T}+Q_{k-1 / k},
\end{aligned}
$$

Smoother Analysis Step:

$$
\begin{aligned}
& d_{k}=y_{k}-H_{k} x_{k / k-1}^{f}, i \in \Sigma_{k} \\
& x_{i \mid k}^{a_{i}}=x_{i \mid k-1}^{a_{i}}+\frac{P_{k}^{f_{i} a_{i}}\left(H_{k}\right)^{i^{T}}}{H_{k}^{i} P_{k}^{f_{i} a_{i}} H_{k}^{i^{T}}+\sigma_{\nu}^{2}} d_{k}, i \in \Sigma_{k} \\
& P_{k, i / k}^{a_{i} a_{i}}=\left(I-\frac{P_{k}^{a_{i} a_{i}}\left(H_{k}\right)^{i^{T}}}{H_{k}^{i} P_{k}^{a_{i} a_{i}} H_{k}^{i^{T}}+\sigma_{\nu}^{2}} H_{k}^{i}\right) \\
& P_{k, i \mid k-1}^{f_{i} a_{i}}, i \in \Sigma_{k} \\
& P_{i / k}^{a_{i}}=P_{i \mid k-1}^{a_{i}}-\frac{P_{k}^{a_{i}}\left(H_{k}\right)^{i^{T}}}{H_{k}^{i} P_{k}^{a_{i}} H_{k}^{i^{T}}+\sigma_{\nu}^{2}} H_{k}^{i} P_{k, i \mid k-1}^{f_{i} a_{i}} \\
& , i \in \Sigma_{k}
\end{aligned}
$$

Notations: Smoothers are initialized as the Kalman filter is. In this case of fix-lag smoothers, for example, while $k$ is lower than the lag $L$, the retrospective analysis is performed only to the $k-1$ previous states. The smoother is initialized with an analysis state vector $x_{0}^{a_{i}}$ and the associated error covariance matrix $P_{0}^{a_{i}}$. The subscript and superscript notations are those of [372]. Superscripts $f$ and $a$ mean 'forecast' and 
'analysis' respectively. $k-1$ and $k$ indicate two consecutive time, $t_{k-1}$ and $t_{k}$, at which observations are available. The subscript notation $k / k-1$ is inherited from the estimation theory. $x_{k / k-1}^{f_{i}}$ represents the forecast state for $i$-th sensor at time $t_{k}$, i.e. the state estimate at time $t_{k}$ given the observations up to time $t_{k-1} \cdot x_{k / k}^{a_{i}}$ is the analysis state of $i$-th sensor at time $t_{k}$.i.e the state estimate at time $t_{k}$ given the observations up to time $t_{k}$. For smoother, the analysis state at $t_{I}$ that includes information of all observations till time $t_{k}$ is noted $x_{I, k}^{a_{i}}$, for $i$-th sensor $P_{k / k-1}^{f_{i}}$ and $P_{k / k}^{a_{i}}$ are the associated state error covariance matrices at $i$-th sensor. Equation (4.7), (4.8) and (4.9) perform the propagation between times $t_{k-1}$ and $t_{k}$. They involve the linear, dynamical model $\Phi_{k-1, k}$ and the model error covariance matrix $Q_{k-1, k}$. The equations (4.10)-(4.13) perform the observational updates of the state estimate and error statistics at time $t_{k}$. They use the observation vector $y_{k}$, the observation error covariance matrix $\sigma_{v}$, the observation operator $H_{k}$. The innovation $d_{k}$ is internally defined. $\Sigma_{k}$, the set of time, indices at which the retrospective anaylysis is produced, i.e. the ensemble that $I$ must span when the observations at $t_{k}$ is considered. The nature of $\Sigma_{k}$ determines the type of smoother. If $\Sigma_{k}$ is a singleton, it corresponds to the fixed-point smoother. If $\Sigma_{k}$ is a fixed and homogeneous series, $\Sigma_{k}=\{0,1, \ldots ., M-1, M\}$ for instance, the smoother is of the fixed-interval type. Note that in the paper $i$ is used for the $i$-th sensor and $k$ is used for the span of observations.

For every sensor subsystem of system (4.3) with multiple sensors, using [373], we can obtain the local Kalman filter $\hat{x}_{1}^{(i)}(t \mid t)$ for the reduced-order state $x_{1}(t)$, the filtering error covariance $P_{1}^{(i)}(t \mid t)$, innovation $\varepsilon^{(i)}(t)$ with covariance $Q_{\varepsilon^{(i)}}(t)$ and the white 
noise filter $\hat{w}(t \mid t)$, and smoothing $P^{S_{(i)}}(i \mid k)$. So, from (4.4), we have the filter of the reduced-order state $x_{2}(t)$ as:

$$
\hat{x}_{2}^{(i)}(k \mid k)=B \hat{x}_{1}^{(i)}(k \mid k)+C \hat{\omega}^{(i)}(k \mid k)
$$

\subsubsection{COMPUTATION OF CROSS COVARIANCE}

From (4.4)-(4.14), we can obtain the Kalman-like particle filter based filtering and smoothing error equations as follows:

$$
\begin{aligned}
& \tilde{x}_{1}^{(i)}(k+1 \mid k)=\bar{\Phi}_{0}^{(i)}\left[I_{n_{1}}-\frac{P_{k}^{i}\left(H_{k}\right)^{i^{T}}}{H_{k}^{i} P_{k}^{i} H_{k}^{i^{T}}+\sigma_{\nu}^{2}} \bar{H}^{(i)}\right] \tilde{x}_{1}^{(i)}(k \mid k-1) \\
& +\Gamma_{0} \omega_{k}-\left(\bar{\Phi}_{0}^{(i)} \frac{P_{k}^{i}\left(H_{k}\right)^{i^{T}}}{H_{k}^{i} P_{k}^{i} H_{k}^{i^{T}}+\sigma_{\nu}^{2}}+J^{i}\right) \eta_{k}^{(i)} \\
& \tilde{x}_{1}^{(i)}(k \mid k)=\left[I_{n_{1}}-\frac{P_{k}^{i}\left(H_{k}\right)^{i^{T}}}{H_{k}^{i} P_{k}^{i} H_{k}^{i^{T}}+\sigma_{\nu}^{2}} \bar{H}^{(i)}\right] \tilde{x}_{1}^{(i)}(k \mid k-1) \\
& \left.-\frac{P_{k}^{i}\left(H_{k}\right)^{i^{T}}}{H_{k}^{i} P_{k}^{i} H_{k}^{i^{T}}+\sigma_{\nu}^{2}}\right) \eta_{k}^{(i)} \\
& \tilde{x}_{2}^{(i)}(k \mid k)=\Phi_{k}^{(i)} \tilde{x}_{1}^{(i)}(k \mid k-1)+D^{(i)}(k)\left[\omega_{k}^{T}, \eta_{k}^{(i)^{T}}\right]^{T}
\end{aligned}
$$

where $\tilde{x}_{1}^{(i)}(k \mid k-1)=x_{1}(k)-\hat{x}_{1}^{(i)}(k \mid k-1), \tilde{x}_{1}^{(i)}(k \mid k)=x_{1}(k)-\hat{x}_{1}^{(i)}(k \mid k), \tilde{x}_{2}^{(i)}(k \mid k)=x_{2}(k)-$ $\hat{x}_{2}^{(i)}(k \mid k), \bar{\Phi}_{0}^{(i)}=\Phi_{0}-J^{(i)} \bar{H}^{(i)}, J^{(i)}=\Gamma_{0} S^{(i)} Q_{\eta^{(i)}}^{-1}, \Phi_{k}^{i}=B\left(I_{n_{1}}-\frac{P_{k}^{i}\left(H_{k} i^{i}\right.}{H_{k}^{i} P_{k}^{i} H_{k}^{i^{T}}+\sigma_{\nu}^{2}} \bar{H}^{i}\right)-$ $C S^{(i)} Q_{\varepsilon^{(i)}}^{-1}(k) \bar{H}^{(i)}$ and $D^{(i)}=\left[C-B \frac{P_{k}^{i}\left(H_{k}\right)^{T}}{H_{k}^{i} P_{k}^{i} H_{k}^{i^{T}}+\sigma_{\nu}^{2}}-C S^{(i)} Q_{\varepsilon^{(i)}}^{-1}(k)\right] . I_{n_{1}}$ is an $n_{1} \times n_{1}$ identity matrix. Using (4.15)-(4.17), and projection theory, the following Lemmas 4.1, 4.2 and 4.3 can be obtained for prediction, filtering and smoothing respectively. 


\section{Cross covariance of Prediction and Filtering Errors}

Lemma 4.1 For system (4.4) with multiple sensors, the cross-covariance matrices of prediction and filtering errors for state $x_{1}(k)$ between the ith and the jth sensor subsystems are given by (See Eqn. (4.18)-(4.19)):

$$
\begin{aligned}
& P_{1}^{i j}(k+1 \mid k)=\bar{\Phi}_{0}^{(i)}\left[I_{n_{1}}-\frac{P_{k}^{i}\left(H_{k}\right)^{i^{T}}}{H_{k}^{i} P_{k}^{i} H_{k}^{i^{T}}+\sigma_{\nu}^{2}} \bar{H}^{(i)}\right] P_{1}^{(i j)}(k \mid k-1) \\
& {\left[I_{n_{1}}-\frac{P_{k}^{j}\left(H_{k}\right)^{j^{T}}}{H_{k}^{j} P_{k}^{j} H_{k}^{j^{T}}+\sigma_{\nu}^{2}} \bar{H}^{(j)}\right]^{T}+\left[\Gamma_{0}-\bar{\Phi}_{0}^{(i)} \frac{P_{k}^{i}\left(H_{k}\right)^{i^{T}}}{H_{k}^{i} P_{k}^{i} H_{k}^{i^{T}}+\sigma_{\nu}^{2}}\right.} \\
& \left.-J^{(i)}\right] Q^{(i j)}\left[\Gamma_{0}-\bar{\Phi}_{0}^{(j)} \frac{P_{k}^{j}\left(H_{k}\right)^{j^{T}}}{H_{k}^{j} P_{k}^{j} H_{k}^{j^{T}}+\sigma_{\nu}^{2}}-J^{(j)}\right]^{T} \\
& P_{1}^{i j}(k \mid k)=\left[I_{n_{1}}-\frac{P_{k}^{i}\left(H_{k}\right)^{i^{T}}}{H_{k}^{i} P_{k}^{i} H_{k}^{i^{T}}+\sigma_{\nu}^{2}} \bar{H}^{(i)}\right] P_{1}^{(i j)}(k \mid k-1) \\
& {\left[I_{n_{1}}-\frac{P_{k}^{j}\left(H_{k}\right)^{j^{T}}}{H_{k}^{j} P_{k}^{j} H_{k}^{j^{T}}+\sigma_{\nu}^{2}} \bar{H}^{(j)}\right]^{T}+} \\
& \frac{P_{k}^{i}\left(H_{k}\right)^{i^{T}}}{H_{k}^{i} P_{k}^{i} H_{k}^{i^{T}}+\sigma_{\nu}^{2}} Q_{\eta^{(i, j)}} \frac{P_{k}^{j}\left(H_{k}\right)^{j^{T}}}{H_{k}^{j} P_{k}^{j} H_{k}^{j^{T}}+\sigma_{\nu}^{2}}
\end{aligned}
$$

with the initial value $P_{1}^{i, j}(0 \mid-1)=P_{01}$ where $P_{01}$ is the first $n_{1} \times n_{1}$ block of $R^{-1} P_{0} R^{-T}$.

Lemma 4.2 For system (4.5) with multiple sensors, the covariance matrix of the filtering errors for state $x_{2}(k)$ between the ith and the jth sensor subsystems is given by (See Eqn. (4.20)):

$$
\begin{aligned}
& P_{2}^{(i j)}(k \mid k)=\Phi^{(i)}(k) P_{1}^{(i j)}(k \mid k-1) \Phi^{(j)^{T}}(k)+D^{(i)}(k) Q^{(i j)} \\
& D^{(j)^{T}}(k)
\end{aligned}
$$

where $P_{2}^{(i i)}(k \mid k)$ is the filtering error covariance of $x_{2}(k)$ based on the $i$-th sensor i.e. 
$P_{2}^{(i)}(k \mid k)$

\section{Cross covariance of Smoothing}

Lemma 4.3 For system (4.4)-(4.5) with multiple sensors, the covariance of smoothing between the $i$-th and $j$-th subsystem are given by:

$$
\begin{aligned}
& \hat{x}(i \mid k)=\hat{x}(i \mid k-1)+P^{i j}(k \mid k-1) r^{i j}(i \mid K) \\
& r^{i j}(i \mid K)=\bar{\Phi}_{p}^{(i)^{T}}\left[I_{n_{1}}-\frac{P_{k}^{i j}\left(H_{k}\right)^{j^{T}}}{H_{k}^{i} P_{k}^{i j} H_{k}^{j^{T}}+\sigma_{\nu}^{2}} \bar{H}^{(j)}\right] r(i+1 \mid K)+H^{(j)^{T}} \\
& {\left[H^{(j)} P^{i j}(k \mid k-1) H^{(j)^{T}}+R(k)\right]^{-1}\left(\tilde{y}_{k+1}^{(j)}-\tilde{H}_{k+1}^{(j)} \tilde{x}_{k+1}^{(j)}\right)} \\
& P^{i j}(k, i \mid T)=P^{i j}(k \mid k-1)-P^{i j}(k \mid k-1) \\
& P^{S_{i j}}(i \mid K) P^{i j}(k \mid k-1) \\
& P^{S_{i j}}(i \mid K)=\bar{\Phi}_{p}^{(i)^{T}}\left[I_{n_{1}}-\frac{P_{k}^{i j}\left(H_{k}\right)^{i^{T}}}{H_{k}^{i} P_{k}^{i j} H_{k}^{j^{T}}+\sigma_{\nu}^{2}} \bar{H}^{(j)}\right]^{T} \\
& P^{S_{i j}}(i+1 \mid K) \bar{\Phi}_{p}^{(j)}\left[I_{n_{1}}-\frac{P_{k}^{i j}\left(H_{k}\right)^{j^{T}}}{H_{k}^{i} P_{k}^{i j} H_{k}^{j^{T}}+\sigma_{\nu}^{2}} \bar{H}^{(i)}\right] \\
& +H^{(i)^{T}}\left[H^{(j)} P(i \mid k-1) H^{(j)^{T}}+R_{k}\right]^{-1} H^{(j)}
\end{aligned}
$$

where $k=N-1, N-2, \ldots, 1$, and $n \times n$ vector $r$ satisfies the backward recursive equation, and $\Phi_{p}(k+1, k)=\Phi(k+1, k)[I-K(k) H(k)]$ and $j=N, N-1, \ldots, 1$ and $r(N+1 \mid N)=0$, also $n \times n$ matrix $P^{S_{i j}}(i \mid K)$, which is the covariance matrix of $r(i \mid K)$ satisfying the backward recursive equation. For state $x_{k}$ between $i$-th and $j$-th 
sensor, the cross-covariance matrices for smoothing are:

$$
\begin{aligned}
P_{1, k}^{\left(S_{i j}\right)}= & I_{n_{1}} P_{1, k}^{a_{i j}} I_{n_{1}}^{T}+\left(H^{(i)} P(j \mid j-1) H^{j^{T}}\right)^{-1} \\
P_{2, k}^{\left(S_{i j}\right)}= & F^{(i)}(k) P_{1, k}^{a_{i j}} F^{j^{T}}(k)+D^{i}(k)\left(H^{i} P(j \mid j-1) H^{j^{T}}\right)^{-1} \\
& D^{\left(i^{T}\right)}
\end{aligned}
$$

where $P_{2, k}^{\left(S_{i i}\right)}(k \mid k)$ is the smoothing error covariance of $x_{2}(k)$ based on the $i$-th sensor i.e. $P_{2}^{\left(S_{i}\right)}(k \mid k)$.

\subsubsection{ESTIMATION OF THE MODEL PARAMETERS USING AN EM}

\section{ALGORITHM}

In this section, we describe the estimation of model parameters with an EM algorithm. The objective is to compute an estimate of $\Theta$, where all the model parameters are denoted by $\Theta=\left\{A, \sigma_{v}^{2}, Q, \mu_{0}, \Sigma_{0}\right\}$. Note that because of the dependence on the states, which are not available, direct maximization is not possible. The problem is to maximize the likelihood with respect to two unknowns: states and model parameters. The EM algorithm takes an iterative approach by first maximizing the likelihood with respect to the states in the $E$-step, and then maximizing with respect to the parameters in the $M$-step. The $E$-step maximum is given by the expected value of the complete log-likelihood function as follows:

$$
Q=E_{X \mid Y}\left[\log p\left(Y_{1: K} X_{1: K} \mid \Theta\right)\right]
$$


where $p\left(Y_{1: K}, X_{1: K} \mid \Theta\right)$ is the probability density function of the measurement, and $Y_{1: K}$ is the a sequence of measurements as $Y_{1: K} \equiv\left\{y_{1}, \ldots ., y_{k}\right\}$. The $M$-step involves the direct differentiation of $Q$ to find the values of the parameters. These computations are done iteratively and convergence of the algorithm is guaranteed [376]. We now describe an EM algorithm for our case to stem it into the full-order Kalman-like particle smoother.

\section{E-Step}

This step involves the computation of $Q$ given the measurements $Y_{1: K}$, which is the future estimation where $K$ is a fixed positive integer. and an estimate of the model parameter from the previous iteration, $\hat{\Theta}_{k}$. The computation of $Q$ depends on the following three quantities:

$$
\begin{aligned}
\hat{x}_{k \mid K}^{a_{i}} & =E\left(x_{k}^{a} \mid Y_{1: K}\right) \\
\Xi_{k \mid K} & =E\left(x_{k}^{a} x_{k}^{a^{T}} \mid Y_{1: K}\right)=P_{k \mid K}+\hat{x}_{k \mid K}^{a} \hat{x}_{k \mid K}^{a^{T}} \\
\Xi_{k, k-1 \mid K} & =E\left(x_{k}^{a} x_{k-1}^{a^{T}} \mid Y_{1: K}\right)=P_{k, k-1 \mid K} \\
& +\hat{x}_{k \mid K}^{a} \hat{x}_{k-1 \mid K}^{a^{T}}
\end{aligned}
$$

where $x_{k}^{a}$ is the value from the smoother analysis step. The first two quantities can be obtained using the Kalman smoother as described in equation (4.11) and (4.13). The last quantity can be obtained with the following equation:

$$
P_{k, k-1 \mid K}=J_{k-1} P_{k \mid K}
$$


where $J_{k}=P_{1, k}^{a_{i}} \Phi_{k-1, k} P_{k, 1 / k-1}^{f_{i} a_{i}{ }^{-1}}$. $Q$ is then obtained using equation (4.33) given in the next section.

Log-Likelihood derivation and $M$-Step : Joint probability distribution of $X_{1: K}$, $Y_{1: K}$ can be written as:

$$
\begin{aligned}
p\left(X_{1: K}, Y_{1: K \mid \Phi}\right) & =p\left(x_{1}^{a}\right) \prod_{k=2}^{K} p\left(x_{k}^{a} \mid x_{k-1}\right) \\
& \cdot \prod_{k=1}^{K} p\left(y_{k} \mid x_{k}^{a}, H_{k}\right)
\end{aligned}
$$

Taking log and expectation, we get the expectation of joint log-likelihood with respect to the conditional expectation:

$$
\begin{aligned}
Q & =E_{X \mid Y}\left[\log p\left(X_{1: K}, Y_{1: K} \mid \Theta\right)\right] \\
& =-\frac{K}{2} \ln \sigma_{v}^{2}-\frac{1}{2 \sigma_{v}^{2}} \sum_{k=1}^{K}\left[y_{k / K}^{2}-2 H_{k}^{T} \widehat{x}_{i / k}^{a_{i}} y_{t}+H_{k}^{T} \Xi_{k \mid K} H_{k}\right] \\
& -\frac{1}{2} \sum_{k=2}^{K} \operatorname{trace}\left[Q ^ { - 1 } \left(\Xi_{k \mid K}-\Phi_{k-1, k} \Xi_{k, k-1 \mid K} \Phi_{k-1, k}{ }^{T}\right.\right. \\
& \left.\left.+\Phi_{k-1, k} \Xi_{k-1 \mid K} \Phi_{k-1, K}^{T}\right)\right] \\
& -\frac{1}{2} \operatorname{trace}\left[V_{1}^{-1}\left(P_{k \mid K}-2 \pi_{1} \widehat{x}_{1}^{a_{i}^{T}}+\pi_{1} \pi_{1}^{T}\right)\right]-\frac{1}{2} \ln \left|V_{1}\right| \\
& -\frac{K-1}{2} \ln |Q|-\frac{(p+1) K}{2} \ln 2 \pi
\end{aligned}
$$

where $y_{k}$ is a particular output measurement at instant $K$. For $M$-step, we take the derivative of $Q$ with respect to each model parameter, and set it to zero to get the 
estimate, e.g., an update for $\Phi_{k-1, k}$ can be found as:

$$
\frac{\partial Q}{\partial \Phi}=-\frac{1}{2} \sum_{k=2}^{K}\left[-2 \Xi_{k, k-1 \mid K}+2 \Phi \Xi_{k-1 \mid K}\right]=0
$$

which gives,

$$
\Phi_{k-1, k}^{k+1}=\left(\sum_{k=2}^{K} \Xi_{k, k-1 \mid K}\right)\left(\sum_{k=2}^{K} \Xi_{k-1, K}\right)^{-1}
$$

Updates for other parameters can be obtained similarly.

\section{M-Step}

By direct differentiation of $Q$, we get the following expressions of the model parameter estimates:

$$
\begin{aligned}
\hat{\Phi}_{k-1, k}^{k+1} & =\left(\sum_{k=2}^{K} \Xi_{k, k-1 \mid K}\right)\left(\sum_{k=2}^{K} \Xi_{k-1, K}\right)^{-1} \\
\hat{Q}_{k-1, k}^{k+1} & =\frac{1}{K-1}\left(\sum_{k=2}^{K} \Xi_{k, \mid K}-\hat{\Phi}^{k+1} \sum_{k=2}^{K} \Xi_{k-1, k \mid K}\right) \\
{\hat{\sigma_{v}^{2}}}^{k+1} & =\frac{1}{K} \sum_{k=1}^{K}\left[y_{k}^{2}-2 H_{k}^{T} \widehat{x}_{k}^{a_{i}} y_{k}+H_{k}^{T} \Xi_{k \mid K} H_{k}\right] \\
\hat{\mu}_{1}^{k+1} & =\hat{x}_{1 \mid K}^{a} \\
\hat{\Sigma}_{0}^{k+1} & =\Xi_{1}-\hat{x}_{1 \mid K}^{a_{i}} \hat{x}_{1 \mid K}^{a^{T}}
\end{aligned}
$$

where $k$ denotes the current iteration. We denote all these estimates together as $\hat{\Theta}^{k+1}$.

Both $E$ and $M$ steps are iterated, and convergence is monitored with the conditional 
likelihood function obtained as follows:

$$
\begin{aligned}
\log p\left(Y_{1: K} \mid \hat{\Theta}^{k}\right)= & \sum_{k=1}^{K} \log \left(N \left(H_{k}^{\prime} \hat{x}_{k \mid k-1}, H_{k}^{T} P_{k \mid k-1} H_{k}\right.\right. \\
& \left.\left.+\sigma_{v}^{2}\right)\right)
\end{aligned}
$$

The algorithm is said to have converged if the relative increase in the likelihood at the current time step compared to the previous time is below a certain threshold. The values of $\hat{\Phi}_{k-1 . k}^{k+1}$ and $\hat{Q}_{k-1, k}^{k+1}$ obtained from $M$-step is then fed into the Kalman-like particle smoother resulting in more efficient results.

The above algorithm can be easily extended to multiple measurements. Assuming trials to be i.i.d., the Kalman smoother estimates need to be averaged over all measurement sequences. Substitution in $M$-step equations will then give the estimate of the parameters corresponding to the multiple measurements.

\subsubsection{FulL-ORDER FUSION}

Theorem 4.1 For singular system (4.1) and (4.2) with multiple sensors, we have the distributed full-order optimal fusion filter

$$
\hat{x}^{0}(k \mid k)=\sum_{i=1}^{l} \bar{A}^{(i)}(k) \bar{x}^{(i)}(k \mid k)
$$

The optimal matrix weights $\bar{A}(i)(k), i=1,2$, , l are computed by:

$$
\bar{A}(k)=\Upsilon^{-1}(k) e\left(e^{T} \Upsilon^{-1}(k) e\right)^{-1}
$$


where $\bar{A}(k)=\left[\bar{A}^{(1)}(k),, \bar{A}^{(l)}(k)\right]^{T}$ and $e=\left[I_{n} I_{n}\right]^{T}$ are both $n l \times n$ matrices. $\Upsilon(k)=$ $\left(P^{(i j)}(k \mid k)\right)_{n l \times n l}$ is an $n l \times n l$ matrix. Covariance matrix $P^{(i j)}(k \mid k)$ between $\tilde{x}^{(i)}(k \mid k)$ and $\tilde{x}^{(j)}(k \mid k)$ is computed by:

$$
P^{(i j)}(k \mid k)=R\left[\begin{array}{cc}
P_{1}^{(i j)}(k \mid k) & P_{12}^{(i j)}(k \mid k) \\
P_{21}^{(i j)}(k \mid k) & P_{2}^{(i j)}(k \mid k)
\end{array}\right] R^{T}
$$

where the correlated matrix $P_{12}^{(i j)}(k \mid k)$ between $\tilde{x}_{1}^{(i)}(k \mid k)$ and $\tilde{x}_{2}^{(j)}(k \mid k)$ is computed by:

$$
\begin{aligned}
P_{12}^{i j}(k \mid k) & =\left(I_{n_{1}}-K^{(i)}(k) \bar{H}^{(i)}\right) P_{1}^{(i j)}(k \mid k-1) F^{(j)^{T}}(k) \\
& +\left[0,-K^{(i)}(k)\right] Q^{(i j)} D^{(j)^{T}}
\end{aligned}
$$

with $P_{12}^{(i j)}(k \mid k)=P_{21}^{(j i)^{T}}(k \mid k) \cdot \hat{x}^{(i)}(k \mid k)$ is computed by:

$$
\hat{x}^{(i)}(k \mid k)=R\left[\hat{x}_{1}^{(i)^{T}}(k \mid k), \hat{x}_{2}^{(i)^{T}}(k \mid k)^{T}\right]
$$

and the variance matrix of the optimal fusion filter $\hat{x}^{0}(k \mid k)$ is computed by:

$$
P^{0}(k \mid k)=\left(e^{T} \Upsilon^{-1}(k) e\right)^{-1}
$$

and we have $P^{o}(k \mid k) \leq P^{(i)}(k \mid k), i=1,2,, l$.

Proof. Taking projection on $x(k)=R\left[x_{1}^{T}(k) x_{2}^{T}(k)\right]^{T}$ gives (18). We have the filtering 
error:

$$
\tilde{x}^{(i)}(k \mid k)=R\left[\tilde{x}_{1}^{(i)^{T}}(k \mid k), \tilde{x}_{2}^{(i)^{T}}(k \mid k)\right]^{T}
$$

From (4.48) we have the covariance matrix of the filtering errors as (4.44). Using (4.16) and (4.17) gives (4.45). Using the optimal fusion algorithm[14], we have (4.42), (4.43), and (4.44). It should be noted that theorem 4.1 sets for the filter, and the theorem 4.2 sets for the smoother.

Theorem 4.2 For singular system (4.1) and (4.2) with multiple sensors, we have the distributed full-order optimal fusion smoother

$$
\hat{x}^{S_{0}}(k \mid k)=\sum_{i=1}^{l} \bar{A}^{(i)}(k) \bar{x}^{\left(S_{i}\right)}(k \mid k)
$$

In case of the full-order smoother fusion, all the other formulation is same except the covariance matrix. Covariance matrix $P^{\left(S_{i j}\right)}(k \mid k)$ between $\tilde{x}^{\left(S_{i}\right)}(k \mid k)$ and $\tilde{x}^{\left(S_{j}\right)}(k \mid k)$ is computed by:

$$
P^{\left(S_{i j}\right)}(k \mid k)=R\left[\begin{array}{cc}
P_{1}^{\left(S_{i j}\right)}(k \mid k) & P_{12}^{\left(S_{i j}\right)}(k \mid k) \\
P_{21}^{\left(S_{i j}\right)}(k \mid k) & P_{2}^{\left(S_{i j}\right)}(k \mid k)
\end{array}\right] R^{T}
$$

where the correlated matrix $P_{12}^{\left(S_{i j}\right)}(k \mid k)$ between $\tilde{x}_{1}^{\left(S_{i}\right)}(k \mid k)$ and $\tilde{x}_{2}^{\left(S_{j}\right)}(k \mid k)$ is computed 
by:

$$
\begin{aligned}
P_{12}^{S_{i j}}(k \mid k) & =\left(I_{n_{1}}\right) P_{1}^{\left(S_{i j}\right)}(k \mid k-1) F^{(j)^{T}}(k) \\
& +\left(\left[0,-H_{k}^{(i)}\right] P^{\left(S_{i j}\right)} D^{(j)^{T}}\right)^{-1}
\end{aligned}
$$

with $P_{12}^{\left(S_{i j}\right)}(k \mid k)=P_{21}^{\left(S_{j i}\right)^{T}}(k \mid k)$.

EM-based full-order Kalman smoother is summarized in Table 4.1.

\subsection{REDUCED-ORDER EM-BASED SEEK SMOOTHER}

Theorem 4.1 gives a distributed full-order optimal fusion Kalman filter. It requires the inverse of an $n l \times n l$ high dimension matrix $\Upsilon(t)$. To reduce the computational burden, we will give a distributed reduced-order fusion Kalman filter.

\subsubsection{SEEK SMOOTHER: A REDUCED-ORder KALMAN}

The SEEK filter is a Kalman filter in which the dimension of the state error space is reduced. It is designed to be applied to large systems. It was founded by Pham [377], based on earlier ideas of Cohn and Todling [378][379], and Verlaan and Heemink [380]. The integration of the matrix $P$, where $P$ comes from the propagation of error covariance matrix is made possible by the order reduction. This matrix is real and symmetric (thus Hermitian), and is therefore diagonalizable, with real eigenvalues and 
Table 4.1: Equations of the EM-Based Kalman-Like Particle Smoother

\section{Initialization :}

$x_{0}^{a}: \frac{P_{k \mid k-1}^{f_{i}}(0) H_{k}(0)^{T}}{H_{k}(0) P_{k \mid k-1}^{f_{i}}(0)\left(H_{k}(0)\right)^{T}+\sigma_{v}^{2}} y_{k}(0)$ and $P_{0}^{a}$

Forecast Step :

$x_{k / k-1}^{f_{i}}=\Phi_{k-1, k} x_{k-1 / k-1}^{a_{i}}$

$P_{k / k-1}^{f_{i}}=\Phi_{k-1 / k}\left(P_{k, k-1 \mid k-1}^{f_{i} a_{i}}\right)^{T}+Q_{k-1 / k}$

State Propagation

Filter Analysis Step :

$G_{k}=H_{k}\left(H_{k} P_{k \mid k-1}^{a}\right)^{T}+Q_{k-1, k}$

$d_{k}=y_{k}-H_{k} x_{k \mid k-1}^{f}$

$x_{k \mid k}^{a_{i}}=x_{k \mid k-1}^{a_{i}}+\frac{P_{k}^{f_{i} a_{i}}\left(H_{k}\right)^{i^{T}}}{H_{k}^{i} P_{k}^{f_{i} a_{i}} H_{k}^{i^{T}}+\sigma_{\nu}^{2}} d_{k}, i \in \Sigma_{k}$

$P_{k / k}^{a_{i}}=P_{k \mid k-1}^{a_{i}}-\frac{P_{k}^{a_{i}}\left(H_{k}\right)^{i}}{H_{k}^{i} P_{k}^{a_{i}} H_{k}^{i^{T}}+\sigma_{\nu}^{2}} H_{k}^{i} P_{k, I \mid k-1}^{f_{i} a_{i}}, i \in \Sigma_{k}$

Error Propagation

Smoother Analysis Step :

$x_{i \mid k}^{a_{i}}=x_{i \mid k-1}^{a_{i}}+\frac{P_{k}^{f_{i} a_{i}}\left(H_{k}\right)^{i^{T}}}{H_{k}^{i} P_{k}^{f_{i} a_{i}} H_{k}^{T^{T}}+\sigma_{\nu}^{2}} d_{k}, i \in \Sigma_{k}$

Smoother analysis

$S_{i \mid k}^{a}=S_{i \mid k-1}^{a}\left[I+\Gamma_{k}\right]^{-1 / 2}, i \in \Sigma_{k}$

Smoother analysis (cov.)

E-Step:

Computation of $Q$ quantities

$\hat{x}_{k \mid K}=E\left(x_{k}^{a} \mid Y_{1: K}\right)$

$\Xi_{k \mid K}=E\left(x_{k}^{a} x_{k}^{a^{T}} \mid Y_{1: K}\right)=P_{k \mid K}+\hat{x}_{k \mid K}^{a} \hat{x}_{k \mid K}^{a^{T}}$

$\Xi_{k, k-1 \mid K}=E\left(x_{k} x_{k-1}^{a^{T}} \mid Y_{1: K}\right)=P_{k, k-1 \mid K}+\hat{x}_{k \mid K}^{a} \hat{x}_{k-1 \mid K}^{a^{T}}$

M-Step :

Direct Differentiation of $Q$,

model parameters

$\hat{\Phi}_{k, k-1}^{k+1}=\left(\sum_{k=2}^{K} \Xi_{k, k-1 \mid K}\right)\left(\sum_{k=2}^{K} \Xi_{k-1, K}\right)^{-1}$

$\hat{Q}^{k+1}=\frac{1}{K-1}\left(\sum_{k=2}^{K} \Xi_{t, \mid T}-\hat{\Phi}_{k, k-1}^{k+1} \sum_{k=2}^{K} \Xi_{k-1, k \mid K}\right)$

${\hat{\sigma_{v}^{2}}}^{k+1}=\frac{1}{K} \sum_{k=1}^{K}\left[y_{k}^{2}-2 H_{k}^{T} \widehat{x}_{k}^{a} y_{k}+H_{k}^{T} \Xi_{k \mid K} H_{k}\right]$

$\hat{\mu}_{1}^{k+1}=\hat{x}_{1 \mid K}^{a}$

$\hat{\Sigma}_{0}^{k+1}=\Xi_{1}-\hat{x}_{1 \mid K}^{a} \hat{x}_{1 \mid K}^{a^{T}}$

Innovation Error Covariance Matrix

Innovation

Filter analysis

Filter analysis (cov.)

Full-Order Fusion :

$P^{(i j)}(k \mid k)$

Filtering covariance Matrix

$P^{\left(S_{i j}\right)}(k \mid k)$

Smoothing covariance Matrix 
orthogonal eigen vectors. It can be written as:

$$
P=N B N^{T}
$$

where $B$ is a diagonal matrix of order $n$ ( $n$ being the dimension of the dynamical system) containing the eigenvalues of $P$ and $N$ is a matrix containing its eigenvectors. The reduction of order consists of usually only a small number $r$ of eigenvectors for expressing $P$, i.e., using a matrix $N$ of order $n \times r$ rather than $n \times n$.

In this section, SEEK-like particle smoother is derived using [372]. To establish the SEEK smoother equations, we proceed recursively i.e. starting from the outputs of a Kalman filter analysis at a time $t_{k-1}$, we apply the smoother forecast and analysis equations at the observation time $t_{k}$. We elaborate the generalized SEEK smoother equations here.

\section{Forecast Step}

Introducing the square-root decomposition of the Kalman filter anaylysis covariance matrix, the smoother forecast equations (4.7)-(4.9) yield:

$$
\begin{aligned}
x_{k / k-1}^{f} & =\Phi_{k-1, k} x_{k-1 \mid k-1}^{a} \\
P_{k, k-1 \mid k-1}^{f a} & =\Phi_{k-1, k} S_{k-1 \mid k-1}^{a} S_{k-1 \mid k-1}^{a}{ }^{T} \\
& =S_{k \mid k-1}^{f} S_{k-1 \mid k-1}^{a}{ }^{T} \\
P_{k \mid k-1}^{f} & =\Phi_{k-1, k} S_{k-1 \mid k-1}^{a} S_{k \mid k-1}^{f}{ }^{T} \\
& =S_{k \mid k-1}^{f} S_{k \mid k-1}^{f} T^{T}
\end{aligned}
$$


where $S_{k \mid k-1}^{f}$ is defined by $S_{k \mid k-1}^{f}=\Phi_{k-1, k} S_{k-1 \mid k-1}^{a}$. It should be noted that the crosscovariance matrix $P_{k, k-1 \mid k-1}^{f a}$ is determined only from the outputs of the filter.

\section{Analysis Step}

We now focus on the smoother analysis components.

Smoother State: The smoothed state $x_{k-1 \mid k}^{a}$ is directly computed using the equation (4.11).

Analysis covariance: Introducing the decompositions of $P_{k-1 \mid k-1}^{a}$ and $P_{k, k-1 \mid k-1}^{f a}$, into the smoother equation (4.13), we compute:

$$
\begin{aligned}
P_{k-1 \mid k}^{a} & =S_{k-1 \mid k-1}^{a}\left(S_{k-1 \mid k-1}^{a}\right)^{T} \\
& -\frac{P_{k-1 \mid k} H_{k}^{T}}{\left(H_{k} P_{k-1 \mid k} H_{k}^{T}+\sigma_{v}^{2}\right)} \cdot H_{k} S_{k \mid k-1}^{f}\left(S_{k-1 \mid k-1}^{a}\right)^{T} \\
& =S_{k-1 \mid k-1}^{a}\left(S_{k-1 \mid k-1}^{a}\right)^{T} \\
& -\frac{S_{k \mid k-1}^{f} S_{k \mid k-1}^{f^{T}} H_{k}^{T} H_{k} S_{k \mid k-1}^{f} S_{k-1 \mid k-1}^{a}}{\left(H_{k} S_{k \mid k-1}^{f} S_{k \mid k-1}^{f^{T}} H_{k}^{T}+\sigma_{v}^{2}\right)} \\
& =S_{k-1 \mid k-1}^{a}\left(I-\frac{\zeta+\sigma_{v}^{2}}{\Gamma}\right)^{-1} S_{k-1 \mid k-1}^{a}{ }^{T}
\end{aligned}
$$

where $\zeta=H_{k} S_{k \mid k-1}^{f} S_{k \mid k-1}^{f^{T}} H_{k}^{T}$, and the Sherman-Morrison-Woodbury formula for matrix inversion is used to derive the SEEK smoother, where Sherman-Morrison-Woodbury 
formula states that:

$$
\begin{aligned}
(A+U D V)^{-1}= & A^{-1}-A^{-1} U\left(D^{-1}+V A^{-1} U\right)^{-1} \\
\cdot & V A^{-1}
\end{aligned}
$$

where $A \equiv R_{k}, U \equiv H_{k} S_{k \mid k-1}^{f}, V \equiv U^{T}$ and $D \equiv I$, the identity matrix.

and $\Gamma_{k}$ is:

$$
\Gamma_{k}=\left(H_{k} S_{k \mid k-1}^{f}\right)^{T} R_{k}^{-1}\left(H_{k} S_{k \mid k-1}^{f}\right)
$$

Now defining

$$
S_{k-1 \mid k}^{a}=S_{k-1 \mid k-1}^{a}\left[I+\Gamma_{k}\right]^{-1 / 2}
$$

a square-root decomposition of the smoother error covariance is obtained.

Analysis cross-covariances: Introducing again the composition (4.54) of $P_{k, k-1 \mid k-1}^{f a}$ from the forecast step, into the smoother expression (4.12) gives:

$$
\begin{aligned}
P_{k, k-1 \mid k}^{a a} & =\left(I-\frac{P_{k \mid k} H_{k}^{T}}{H_{k} P_{k \mid k} H_{k}^{T}+\sigma_{v}^{2}} H_{k}\right) S_{k-1 \mid k-1}^{a}{ }^{T} \\
& =S_{k \mid k-1}^{f}\left[I+\Gamma_{k}\right]^{-1} S_{k-1 \mid k-1}^{a}{ }^{T}
\end{aligned}
$$


and using the definition (4.59), it appears that the cross-covariance matrix $P_{k, k-1 \mid k}^{a a}$ can be decomposed using the square roots of $P_{k \mid k}^{a}$ and $P_{k-1 \mid k}^{a}$ :

$$
P_{k, k-1 \mid k}^{a a}=S_{k \mid k}^{a} S_{k-1 \mid k}^{a}
$$

At the end of the analysis step cycle, the analysis covariance and cross-covariance matrices of the smoother are fully determined with the square root matrices $S_{k \mid k}^{a}$ and $S_{k-1 \mid k}^{a}$

\section{Past states estimates}

The smoothed analysis state vector and square root error covariance matrix are determined for time $t_{k-1}$ given observations at $t_{k}$. The strong point is that the square root matrices not only lead to the covariance matrices, but also provide the cross-covariance matrix. Proceeding then recursively, the smoother estimates $x_{i \mid k}^{a}$ and $S_{i \mid k}^{a}(i<k-$ 1) from the filter estimate $x_{k-1 \mid k-1}^{a}, S_{k-1 \mid k-1}^{a}$, and the smoother estimates $x_{i \mid k-1}^{a}$ and $S_{i \mid k-1}^{a}(i<k-1)$. The smoother equations may be applied involving the smoother estimate at time $t_{i}$. This strictly follows the step of Sections 4.5.1 and 4.5.1. The forecast/analysis cross-covariance is given by:

$$
P_{k, i \mid k-1}^{f a}=\Phi_{k-1, k} S_{k-1 \mid k-1}^{a} S_{i \mid k-1}^{a}{ }^{T}=S_{k \mid k-1}^{f} S_{i \mid k-1}^{a}{ }^{T}
$$

and the square root error covariance matrix of the smoothed estimate are computed as:

$$
S_{i \mid k}^{a}=S_{i \mid k-1}^{a}\left[I+\Gamma_{k}\right]^{-1 / 2}
$$


Finally, it can be verified that the analysis error covariance and cross-covariance matrices are decomposed as:

$$
\begin{aligned}
P_{i \mid k}^{a} & =S_{i \mid k}^{a} S_{i \mid k}^{a}{ }^{T} \\
P_{k, i \mid k}^{a a} & =S_{k \mid k}^{a} S_{i \mid k}^{a}{ }^{T}
\end{aligned}
$$

This finalizes the full set of the SEEK smoother equations with a perfect model summarized in Table 4.2.

\subsubsection{ESTIMATION OF THE MODEL PARAMETERS USING AN EM}

\section{ALGORITHM}

In this section, we describe the estimation of model parameters for SEEK smother with an EM algorithm. The problem is to maximize the likelihood with respect to two unknowns: states and model parameters. We now describe an EM algorithm for the reduced-order SEEK smoother as follows.

\section{E-Step}

This step involves the computation of $Q$ given the measurements $Y_{1: K}$ and an estimate of the model parameter from the previous iteration, $\hat{\Phi}_{k}$. $Q$ depends on the following 
three quantities:

$$
\begin{aligned}
\hat{x}_{k \mid K} & =E\left(x_{k}^{a} \mid Y_{1: K}\right) \\
\Xi_{k \mid K} & =E\left(x_{k}^{a} x_{k}^{a^{T}} \mid Y_{1: K}\right)=S_{i \mid K}^{a}+\hat{x}_{k \mid K}^{a} \hat{x}_{k \mid K}^{a^{T}} \\
\Xi_{k, k-1 \mid K} & =E\left(x_{k}^{a} x_{k-1}^{a^{T}} \mid Y_{1: K}\right)=S_{k, k-1 \mid K}^{a} \\
& +\hat{x}_{k \mid K}^{a} \hat{x}_{k-1 \mid K}^{a^{T}}
\end{aligned}
$$

The first two quantities can be obtained using the smoother analysis equation smoother analysis (cov.) equation respectively from Table 4.2. The last quantity obtained with the following equation:

$$
S_{i, i-1 \mid k}^{f}=J_{k-1} S_{i \mid k}^{a}
$$

where $J_{k-1}=S_{i \mid k}^{a} \Phi_{k-1, k}^{T} S_{k \mid k-1}^{f}{ }^{-1} \cdot Q$ is then obtained using equation (4.71) given in the next section.

Log-Likelihood derivation and $M$-Step : Joint probability distribution of $X_{1: K}$, $Y_{1: K}$ can be written as:

$$
\begin{aligned}
p\left(X_{1: K}, Y_{1: K \mid \Phi}\right) & =p\left(x_{1}^{a}\right) \prod_{k=2}^{K} p\left(x_{k}^{a} \mid x_{k-1}^{a}\right) \\
& \cdot \prod_{k=1}^{K} p\left(y_{k} \mid x_{k}^{a}, H_{k}\right)
\end{aligned}
$$


Taking log and expectation, we get the expectation of joint log-likelihood with respect to the conditional expectation:

$$
\begin{aligned}
Q & =E_{X \mid Y}\left[\log p\left(X_{1: K}, Y_{1: K} \mid \Theta\right)\right] \\
& =-\frac{K}{2} \ln \sigma_{v}^{2}-\frac{1}{2 \sigma_{v}^{2}} \sum_{k=1}^{K}\left[y_{k}^{2}-2 H_{k}^{T} \widehat{x}_{k}^{a} y_{k}+H_{k}^{T} \Xi_{k \mid K} H_{k}\right] \\
& -\frac{1}{2} \sum_{k=2}^{K} \operatorname{trace}\left[Q ^ { - 1 } \left(\Xi_{k \mid K}-\Phi_{k-1, k} \Xi_{k, k-1 \mid T} \Phi_{k-1, k}^{T}\right.\right. \\
& \left.\left.+\Phi_{k-1, k} \Xi_{k-1 \mid K} \Phi_{k-1, k}^{T}\right)\right] \\
& -\frac{1}{2} \operatorname{trace}\left[V_{1}^{-1}\left(S_{1 \mid K}^{a}-2 \pi_{1} \widehat{x}_{1}^{a^{T}}+\pi_{1} \pi_{1}^{\prime}\right)\right]-\frac{1}{2} \ln \left|V_{1}\right| \\
& -\frac{K-1}{2} \ln |Q|-\frac{(p+1) T}{2} \ln 2 \pi
\end{aligned}
$$

For $M$-step, we take the derivative of $Q$ with respect to each model parameter, and set it to zero to get the estimate, e.g., an update for $\Phi_{k-1, k}$ can be found as:

$$
\frac{\partial Q}{\partial \Phi}=-\frac{1}{2} \sum_{k=2}^{K}\left[-2 \Xi_{k, k-1 \mid K}+2 \Phi_{k-1, k} \Xi_{k-1 \mid K}\right]=0
$$

which gives,

$$
\Phi_{k-1, k}^{k+1}=\left(\sum_{k=2}^{K} \Xi_{k, k-1 \mid K}\right)\left(\sum_{k=2}^{K} \Xi_{k-1, K}\right)^{-1}
$$

Updates for other parameters can be obtained similarly. 


\section{M-Step}

By direct differentiation of $Q$, we get the following expressions of the model parameter estimates:

$$
\begin{aligned}
\hat{\Phi}_{k-1, k}^{k+1} & =\left(\sum_{k=2}^{K} \Xi_{k, k-1 \mid K}\right)\left(\sum_{k=2}^{K} \Xi_{k-1, K}\right)^{-1} \\
\hat{Q}^{k+1} & =\frac{1}{K-1}\left(\sum_{k=2}^{K} \Xi_{k \mid k}-\hat{\Phi}_{k-1, k}^{k+1} \sum_{k=2}^{K} \Xi_{k-1, k \mid K}\right) \\
{\hat{\sigma_{v}^{2}}}^{k+1} & =\frac{1}{K} \sum_{k=1}^{K}\left[y_{k}^{2}-2 H_{k}^{T} \widehat{x}_{k}^{a} y_{k}+H_{k}^{T} \Xi_{k \mid K} H_{k}\right] \\
\hat{\mu}_{1}^{k+1} & =\hat{x}_{1 \mid K}^{a} \\
\hat{\Sigma}_{0}^{k+1} & =\Xi_{1}-\hat{x}_{1 \mid K} \hat{x}_{1 \mid K}^{a^{T}}
\end{aligned}
$$

where $k$ denotes the current iteration. We denote all these estimates together as $\hat{\Theta}^{k+1}$.

Both $E$ and $M$ steps are iterated, and convergence is monitored with the conditional likelihood function obtained as in (4.41).

\subsubsection{REDUCED-ORDER FUSION}

The estimates from SEEK smoother are fed into the $E$-step for computation of $Q$ and $M$-step for direct differentiation of $Q$ and the model estimates. It is an iterative algorithm. Full set of reduced-order EM-based SEEK smoother is summarized in Table 4.2.

Remark 4.5.1 It should be noted that there is no reduced-order fusion step involved in the formulation of Table 4.2 that is EM-based Kalman-like particle SEEK smoother. It 
Table 4.2: Equations of the EM-Based Kalman-Like Particle SEEK Smoother

\section{Initialization :}

$x_{0}^{a}: \frac{S_{k \mid k-1}^{f_{i}}(0) S_{k \mid k-1}^{f_{i}}(0)^{T} H_{k}(0)^{T}}{H_{k}(0) S_{k \mid k-1}^{f_{i}}(0) S_{k \mid k-1}^{f_{i}}(0)^{T}\left(H_{k}(0)\right)^{T}+\sigma_{v}^{2}} y_{k}(0)$ and $S_{0}^{a}$

Forecast Step :

$x_{k / k-1}^{f}=\Phi_{k-1, k} x_{k-1 \mid k-1}^{a}$

$S_{k / k-1}^{f}=\Phi_{k-1, k} S_{k-1 \mid k-1}^{a}$

\section{State Propagation}

Error Propagation

Filter Analysis Step :

$$
\begin{array}{ll}
\Gamma_{k}=\left(H_{k} S_{k \mid k-1}^{f}\right)^{T} R_{k}^{-1}\left(H_{k} S_{k \mid k-1}^{f}\right) & \\
d_{k}=y_{k}-H_{k} x_{k \mid k-1}^{f} & \text { Innovation } \\
x_{k \mid k}^{a}=x_{k \mid k-1}^{f}+S_{K \mid k}^{f}\left[I+\Gamma_{k}\right]^{-1}\left(H_{k} S_{k \mid k}^{f}\right) R_{k}^{-1} d_{k}, i \in \Sigma_{k} & \text { Filter analysis } \\
S_{k \mid k}^{a}=S_{k \mid k-1}^{f}\left[I+\Gamma_{k}\right]^{-1 / 2}, i \in \Sigma_{k} & \text { Filter analysis (cov.) }
\end{array}
$$

\section{Smoother Analysis Step :}

$x_{i \mid k}^{a}=x_{i \mid k-1}^{a}+S_{i \mid k}^{f}\left[I+\Gamma_{k}\right]^{-1}\left(H_{k} S_{i \mid k}^{f}\right) R_{k}^{-1} d_{k}, i \in \Sigma_{k}$

Smoother analysis

$S_{i \mid k}^{a}=S_{i \mid k-1}^{a}\left[I+\Gamma_{k}\right]^{-1 / 2}, i \in \Sigma_{k}$

Smoother analysis (cov.)

E-Step :

Computation of $Q$ quantities

$$
\begin{aligned}
& \hat{x}_{k \mid K}^{a}=E\left(x_{k}^{a} \mid Y_{1: K}\right) \\
& \Xi_{k \mid K}=E\left(x_{k}^{a} x_{k}^{a^{K}} \mid Y_{1: K}\right)=S_{k \mid K}^{a}+\hat{x}_{k \mid K}^{a} \hat{x}_{k \mid K}^{a^{T}} \\
& \Xi_{k, k-1 \mid K}=E\left(x_{k}^{a} x_{k-1}^{a^{T}} \mid Y_{1: K}\right)=S_{k, k-1 \mid K}^{a}+\hat{x}_{k \mid K}^{a} \hat{x}_{k-1 \mid K}^{a^{T}} \\
& \text { M-Step : } \\
& \hat{\Phi}_{k-1, k}^{k+1}=\left(\sum_{k=2}^{K} \Xi_{k, k-1 \mid K}\right)\left(\sum_{k=2}^{K} \Xi_{k-1, K}\right)^{-1} \\
& \hat{Q}^{k+1}=\frac{1}{K-1}\left(\sum_{k=2}^{K} \Xi_{k, \mid K}-\hat{\Phi}_{k-1, k}^{k+1} \sum_{k=2}^{K} \Xi_{k-1, k \mid K}\right) \\
& {\hat{\sigma_{v}^{2}}}^{k+1}=\frac{1}{K} \sum_{k=1}^{K}\left[y_{k}^{2}-2 H_{k}^{\prime} \hat{x}_{k}^{a} y_{k}+H_{k}^{T} \Xi_{k \mid K} H_{k}\right] \\
& \hat{\mu}_{1}^{k+1}=\hat{x}_{1 \mid K}^{a} \\
& \hat{\Sigma}_{0}^{k+1}=\Xi_{1}-\hat{x}_{1 \mid K}^{a} \hat{x}_{1 \mid K}^{a T} \\
&
\end{aligned}
$$

Direct differentiation of $Q$, model parameters 
is the embedded nature of the SEEK filter that it treates the covariance of the system in the form of reduced-order, which was not the case when we were dealing with the basic Kalman smoother as formulated in Table 4.1. In the following section, we will do the evaluation of the proposed smoother schemes.

\subsection{Evaluation AND TESTING}

\subsubsection{DESCRIPTION OF THE POWER QUALITy LAB}

The evaluation and testing has been made on an power lab designed as a utility plant in Electrical Engineering department at KFUPM. The layout of the system can be seen in Fig 4.3. The main idea behind the design of the system is that we have one AC Power source which is considered as a utility, and different units, which are considered to be as the consumers. The purpose of the system is to monitor and measure the voltage and current, and to control the active filter. The following are the units of the set-up:

\section{Programmable AC Source}

There is a programmable AC Source of $18 \mathrm{kVA}$ which is supplying a 3 phase of current and 400 Volts with cycle of 60 Hertz.

\section{Main Panel for Switching}

There is a main panel for switching which connects and controls all the transmission lines, breakers and multiple feeders. 


\section{Active Power Filter}

There is an active power filter which is a 3 phase filters. Its function is to mitigate the harmonics.

\section{Digital Signal Processing(DSP) Filter}

There is a DSP filter. Its function is to implement for active filter.

\section{DSP Setup}

DSP setup is planned basically in the National Instruments Lab-view to implement advance signal processing.

\section{Adjustable Speed Drive(ASD)}

ASD is used for the motor drive implementation. It gives non-linear current because it generate harmonics.

\section{Electronic Loads}

This is an AC/DC electronic load model. We can build any non-linear/dynamic load to the capacity of $1.8 \mathrm{~kW}$ here.

\section{Resistor Bank}

This is a resistive bank for the load. It carries linear load, which has no distortion and harmonics. 


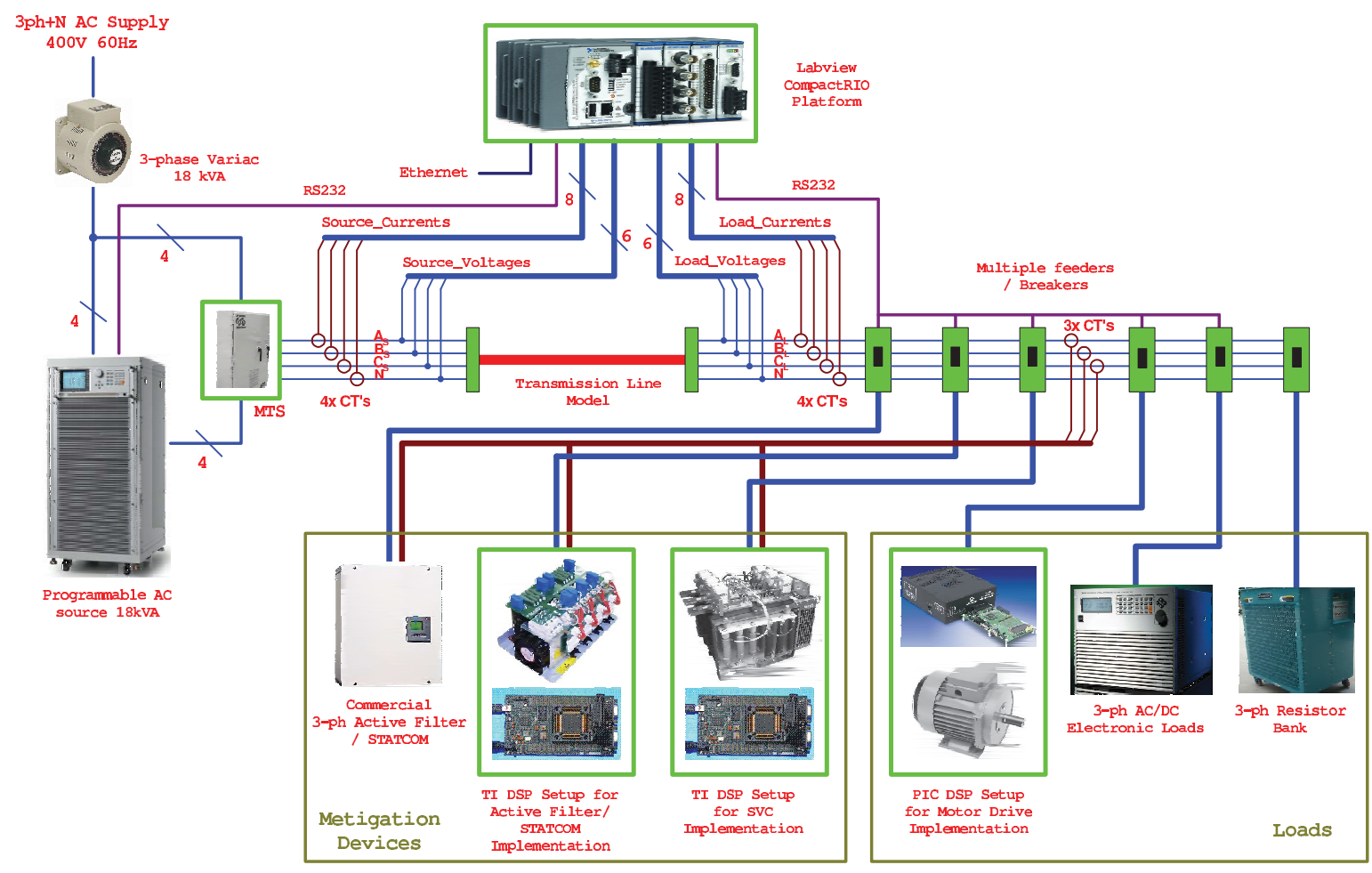

Figure 4.3: Power System Lab

\subsubsection{LOAD SCENARIOS}

Load scenarios are created by using the power quality laboratory. In these scenarios DC motor drive load, linear load and non-linear load fault are being considered.

Scenario I: DC Motor Load In this scenario, while the system is working in real time, DC Motor load is being introduced in the system by using the ASD. With the help of lab-view, we were able to collect the data of the 3 phases of voltage. The data is collected at a fixed sampling time 100 milliseconds.

Scenario II: linear load In this scenario, while the system is working in real time, linear load is being introduced in the system by using the resistor bank. With the help of lab-view, we were able to collect the data of the 3 phases of voltage. The data is 
collected at a fixed sampling time 100 milliseconds.

Scenario III: non-linear load In this scenario, while the system is working in real time, linear load is being introduced in the system by using the electronic loads which is capable of generating dynamic loads. With the help of lab-view, we were able to collect the data of the 3 phases of voltage. The data is collected at a fixed sampling time 100 milliseconds.

\subsubsection{EVALUATION OF RESUlts}

In what follows, we present simulation results for the proposed EM-Based smoother with versions of full and reduced order respectively. The experiments have been performed on the power quality system. Three sets of loads have been considered here, that is, the DC-motor drive load, linear load and non-linear load. Firstly, the data collected from the plant has been initialized and the parameters have been being optimized which comprises of the pre-processing and normalization of the data. The comparison of results for the distributed smoother estimation, and smoother estimation generated from various levels of loads, and the basic profile of that particular load has been compared. Moreover, same pattern of comparison has been followed for full-order and reduced-order showing the effectiveness of the proposed smoother in all cases. 


\section{Load 1 (DC-Motor Drive): Estimates comparison of distributed estimation with}

\section{full-order and reduced order EM-Based Smoothers}

The Kalman-like particle smoother has been simulated here for the DC-motor drive load of the plant. Simulations have been made for the estimate of each case. In the simulation, comparison of various phases of DC-motor drive load i.e. phase 1, phase 2, and phase 3 , and distributed estimation has been shown. It can be seen from the estimate profile in Fig. 4.4 for full-order EM based smoother that the EM results are trying to coop well with the estimates and even the original profile of the load. This is due to

the EM implementation made on the $Q, \sigma_{v}$, and $\hat{\Phi}_{k}$ parameters. When it comes to the reduced-order implementation, it can be seen from Fig. 4.7 for DC motor drive phase 1 load, Fig. 4.8 for DC motor drive phase 2 load and Fig. 4.9 for DC motor drive phase 3 load that reduced-order of the SEEK filter is performing very well as compared to the full-order version of Fig. 4.4. This is due to the reduced-order nature of the filter that it is treating the covariances seperately. In the case of reduced order EM implementation, the estimate is almost over-writing the original profile of the load without estimate, thus showing the effectiveness of the proposed scheme. The distributed version of the reduced-order smoother is even more succinct as can be seen from Fig. 4.10.

\section{Load 2 (Linear): Estimates comparison of distributed estimation with full-order and reduced order EM-Based Smoothers}

In case of load 2, it can be seen for the estimate profile in Fig. 4.5 for full-order EM based smoother that the EM results are trying to coop well with the estimates and even 
the original profile of the load. When it comes to the reduced-order implementation, it can be seen from Fig. 4.11 for linear phase 1 load, Fig. 4.12 for linear phase 2 load and Fig. 4.13 for linear phase 3 load that reduced-order of the SEEK filter is performing very well as compared to the full-order version of Fig. 4.5. The distributed version of the reduced-order smoother is even more succinct as can be seen from Fig. 4.14.

\section{Load 3 (Non-linear): Estimates comparison of distributed estimation with full- order and reduced order EM-Based Smoothers}

In case of non-linear load 3, it can be seen for the estimate profile in Fig. 4.6 for full-order EM based smoother that the EM results are trying to coop well with the estimates and even the original profile of the load. When it comes to the reduced-order implementation, it can be seen from Fig. 4.15 for non-linear phase 1 load, Fig. 4.16 for non-linear phase 2 load and Fig. 4.17 for non-linear phase 3 load that reduced-order of the SEEK filter is performing very well as compared to the full-order version of Fig. 4.6. The distributed version of the reduced-order smoother is even more succinct as can be seen from Fig. 4.18.

\section{Mean Square Error Comparison}

In this section, we have made a comparison of the full versions of both full and reducedorder respectively. Though both versions are having a mean square error value near to zero. But when it comes to precision in the performance, it can be seen from the Fig. 4.19 that how the full-order filter has a dead-end for the reduction of error. After 2 iterations, it almost the same level of mean square error. However, the reduced-order 
Table 4.3: Quantitative Error Comparison Table

\begin{tabular}{|c|c|c|}
\hline ITERATIONS & DISTRIBUTED FULL ORDER & DISTRIBUTED REDUCED ORDER \\
\hline $\bar{~} 1$ & 0.00508 & 0.00525 \\
\hline 2 & 0.00507 & 0.00521 \\
\hline 3 & 0.00507 & 0.00491 \\
\hline 4 & 0.00507 & 0.00489 \\
\hline 5 & 0.00507 & 0.00488 \\
\hline
\end{tabular}

version has better results at every iteration, thus leading almost to a value near to zero at 5 th iteration. The quantitative error comparison can be seen in the Table 4.3. 


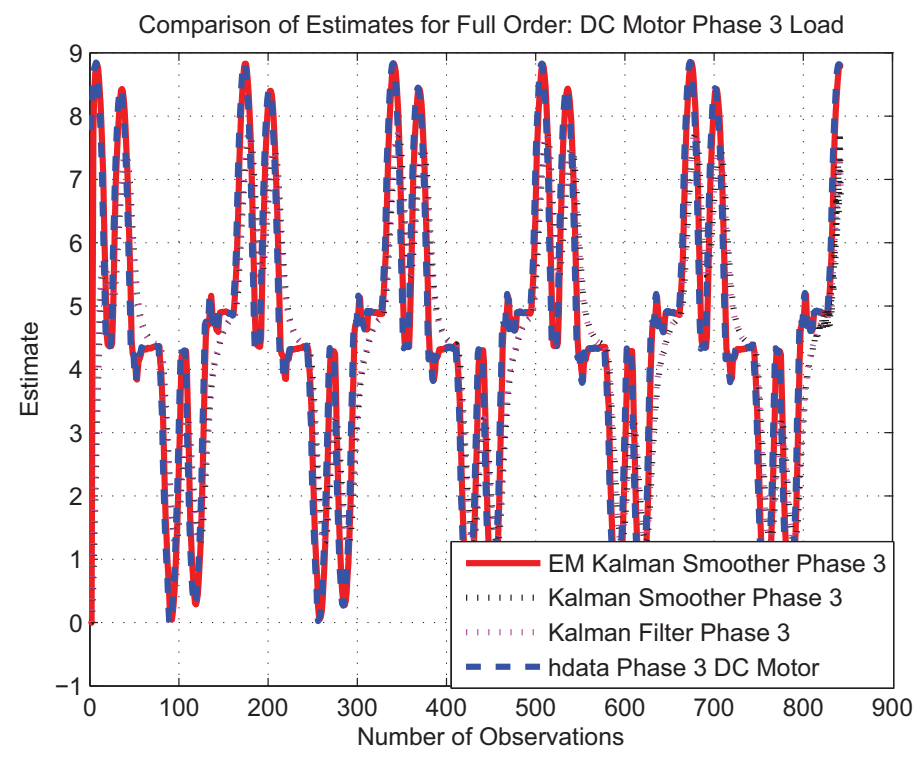

Figure 4.4: Estimates for full-order smoother for Phase 3: DC motor drive Load

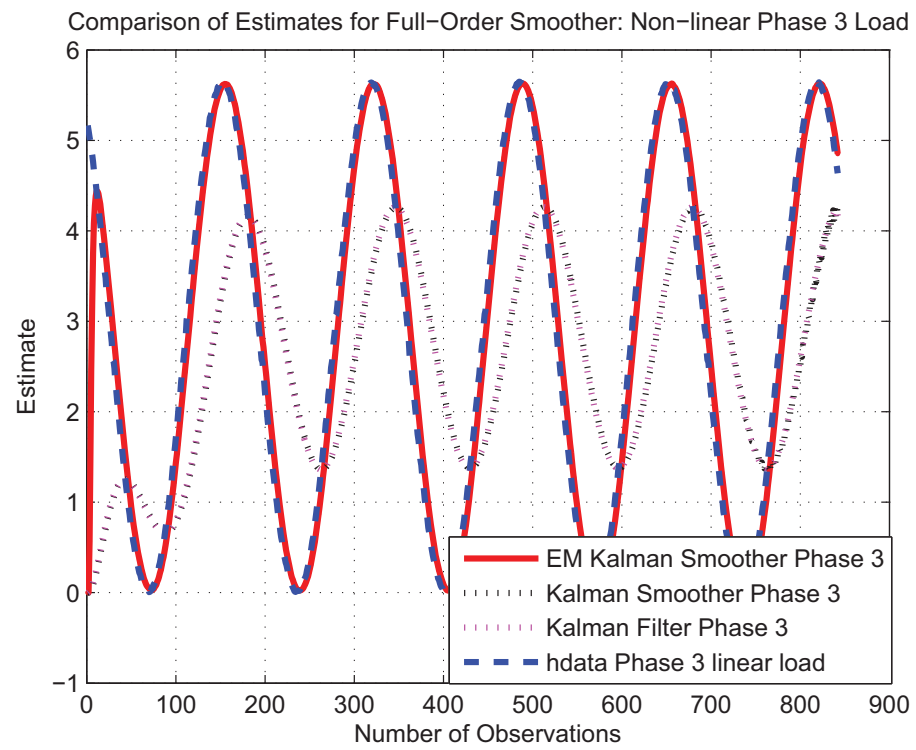

Figure 4.5: Estimates for full-order smoother for Phase 3: Linear Load 


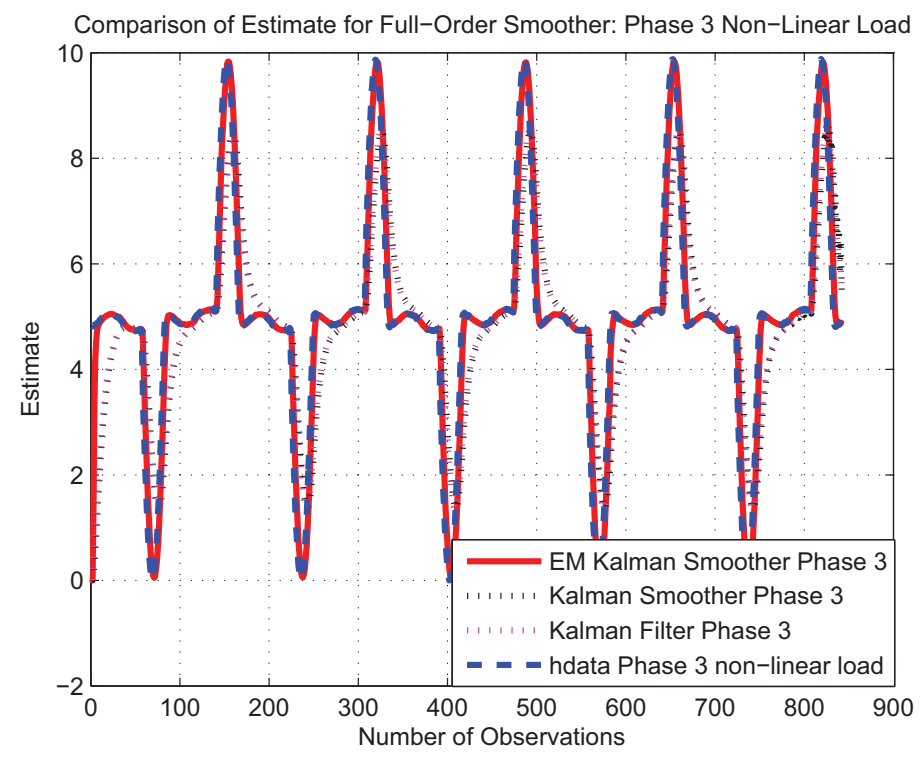

Figure 4.6: Estimates for full-order smoother for Phase 3: Nonlinear Load

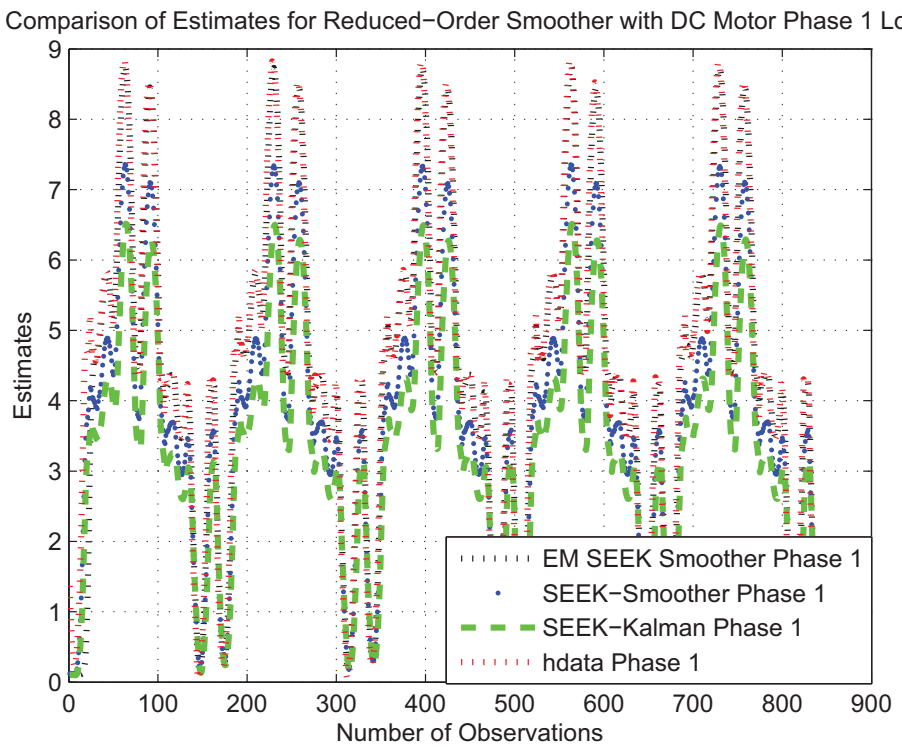

Figure 4.7: Estimates for reduced-order smoother for Phase 1: DC motor drive Load 


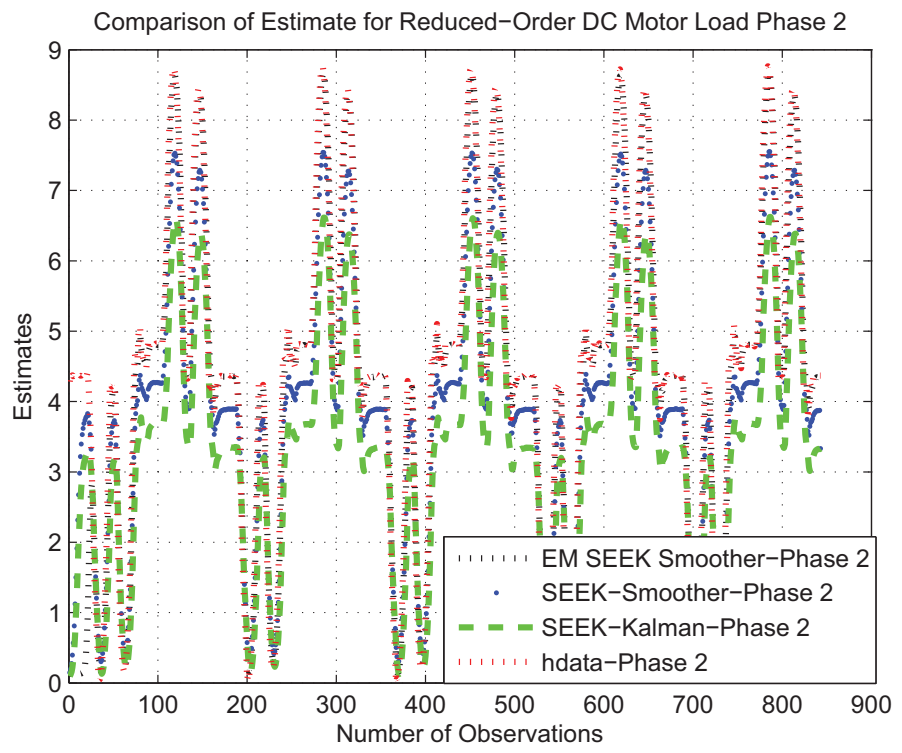

Figure 4.8: Estimates for reduced-order smoother for Phase 2: DC motor drive Load

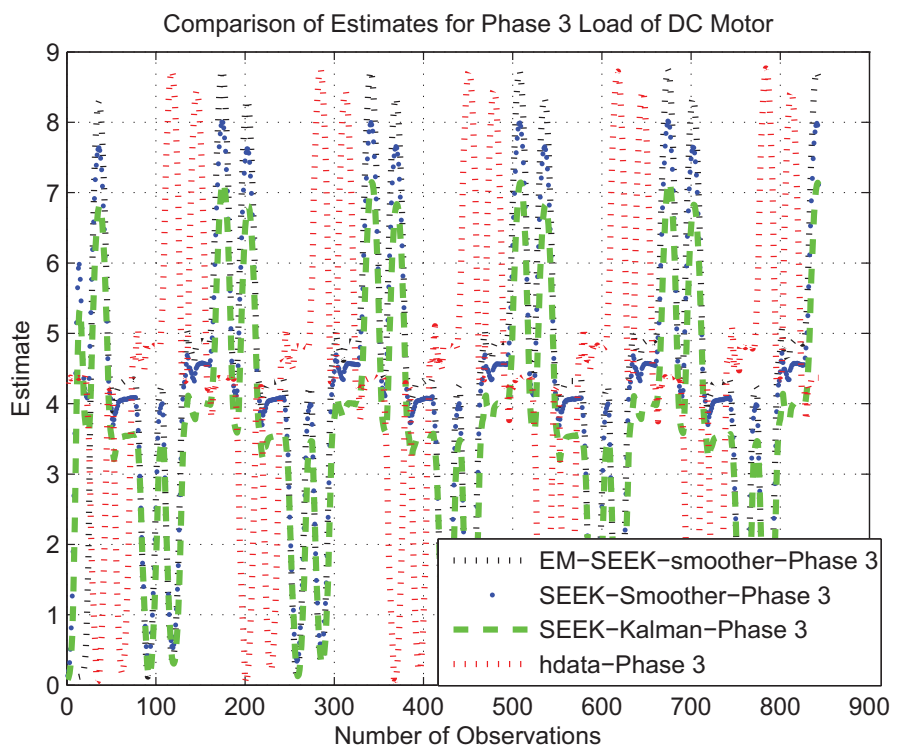

Figure 4.9: Estimates for reduced-order smoother for Phase 3: DC motor drive Load 


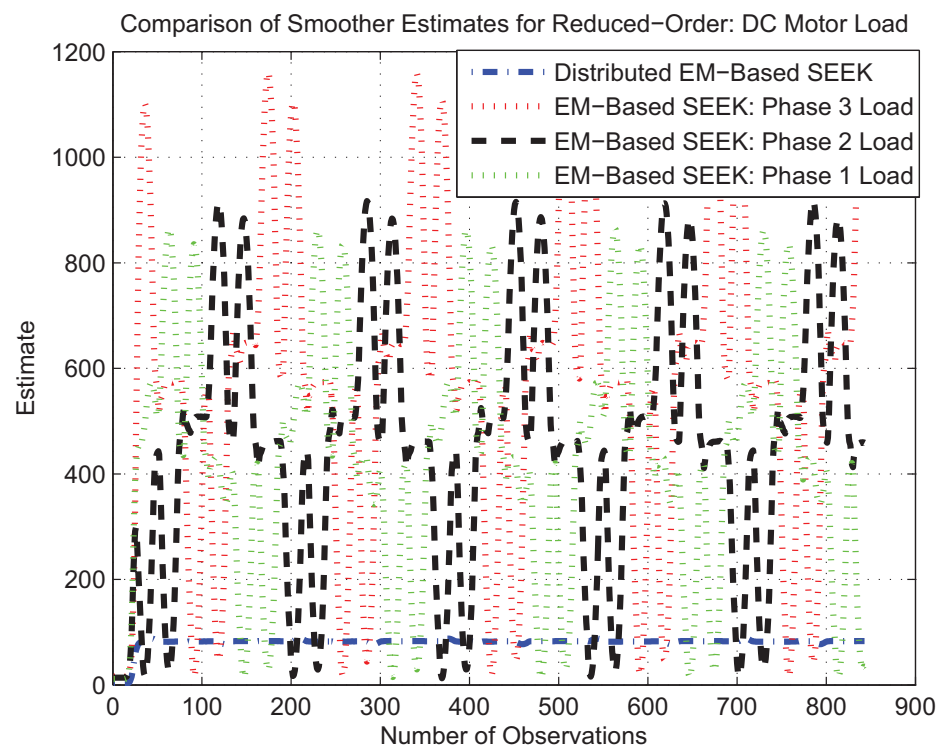

Figure 4.10: Estimates for various reduced-order smoothers: DC motor Load

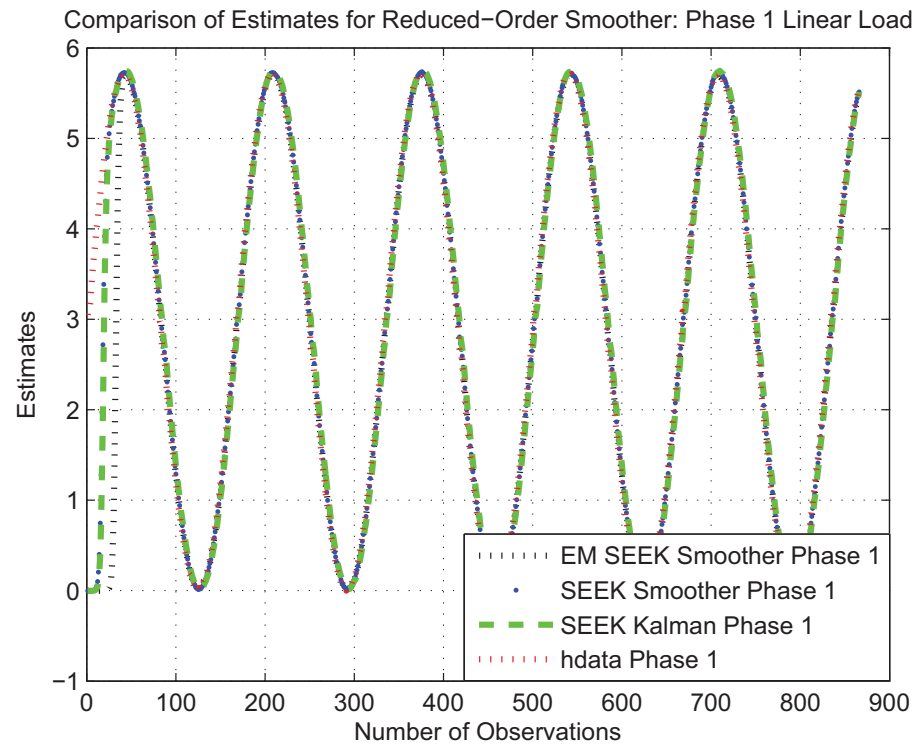

Figure 4.11: Estimates for reduced-order smoother for Phase 1: Linear Load 


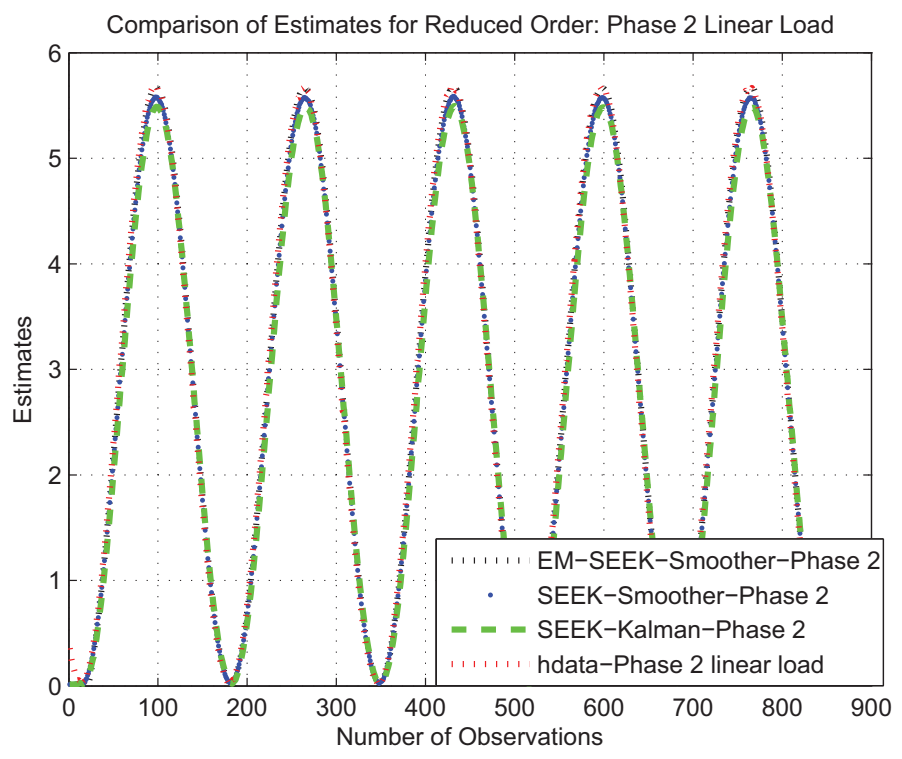

Figure 4.12: Estimates for reduced-order smoother for Phase 2: Linear Load

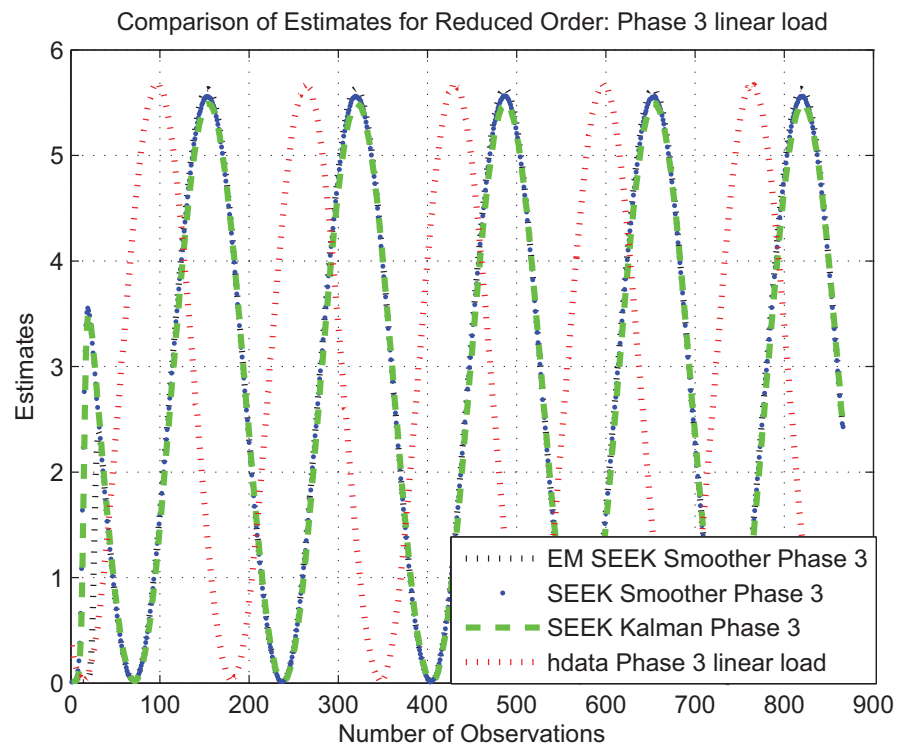

Figure 4.13: Estimates for reduced-order smoother for Phase 3: Linear Load 


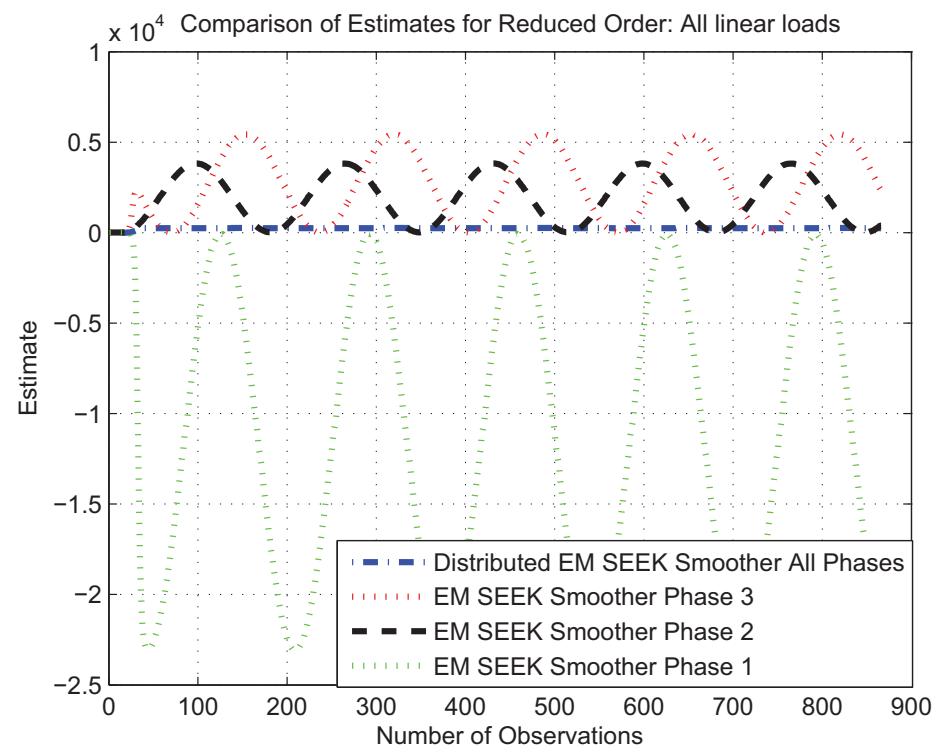

Figure 4.14: Estimates for various reduced-order smoothers: Linear Load

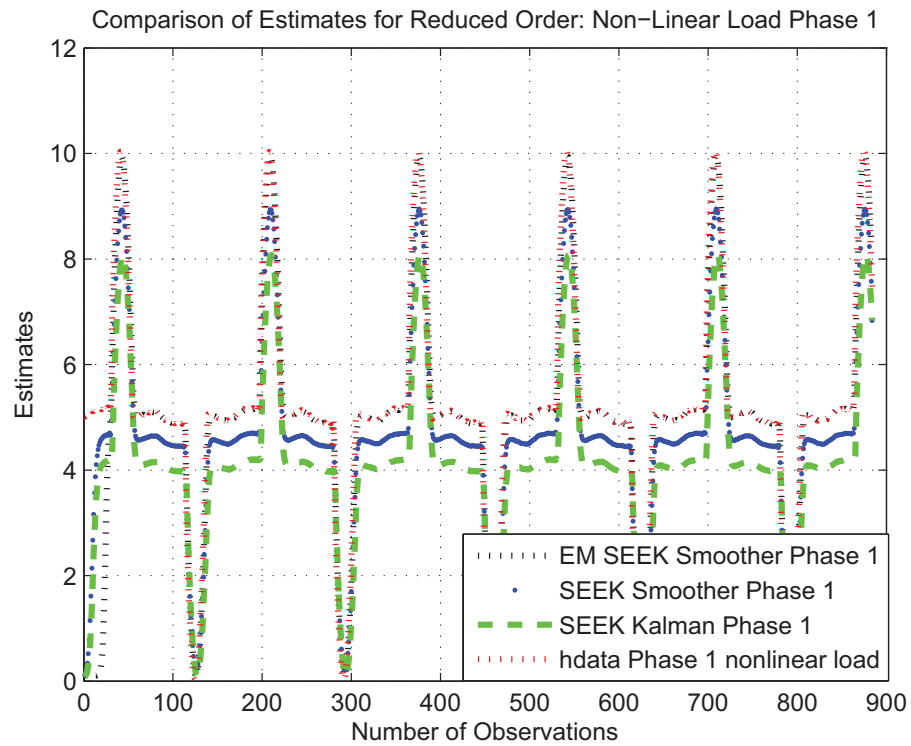

Figure 4.15: Estimates for reduced-order smoother for Phase 1: Nonlinear Load 


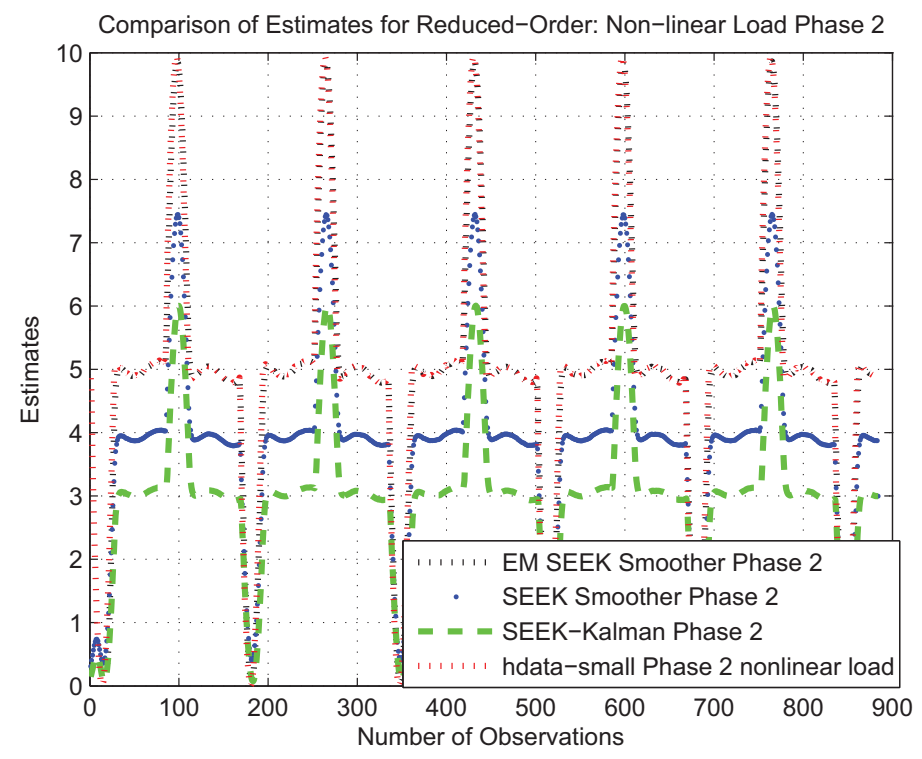

Figure 4.16: Estimates for reduced-order smoother for Phase 2: Nonlinear Load

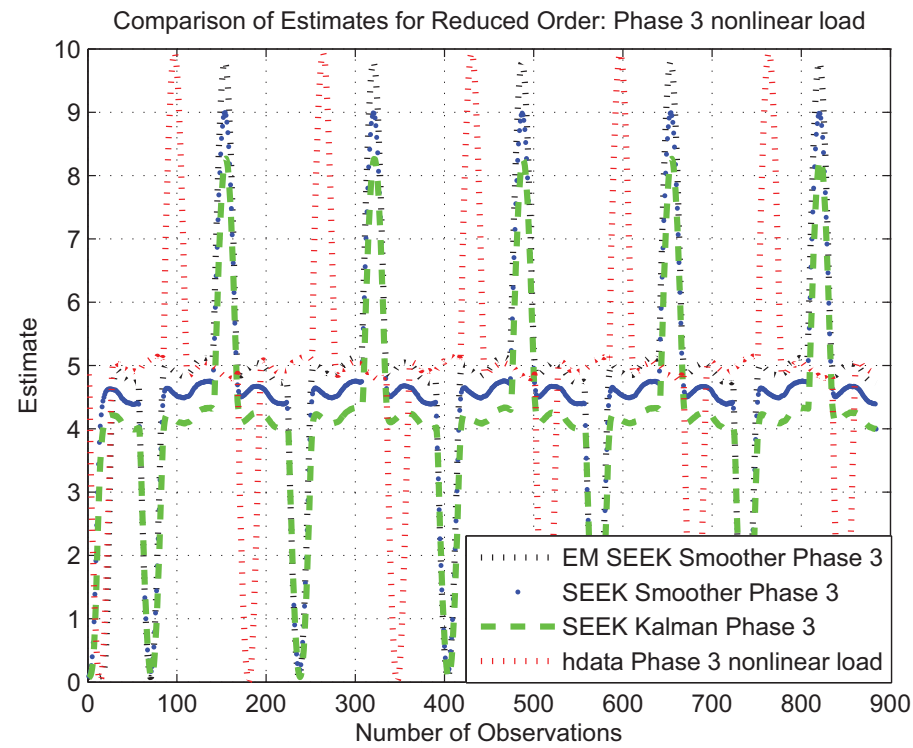

Figure 4.17: Estimates for reduced-order smoother for Phase 3: Nonlinear Load 


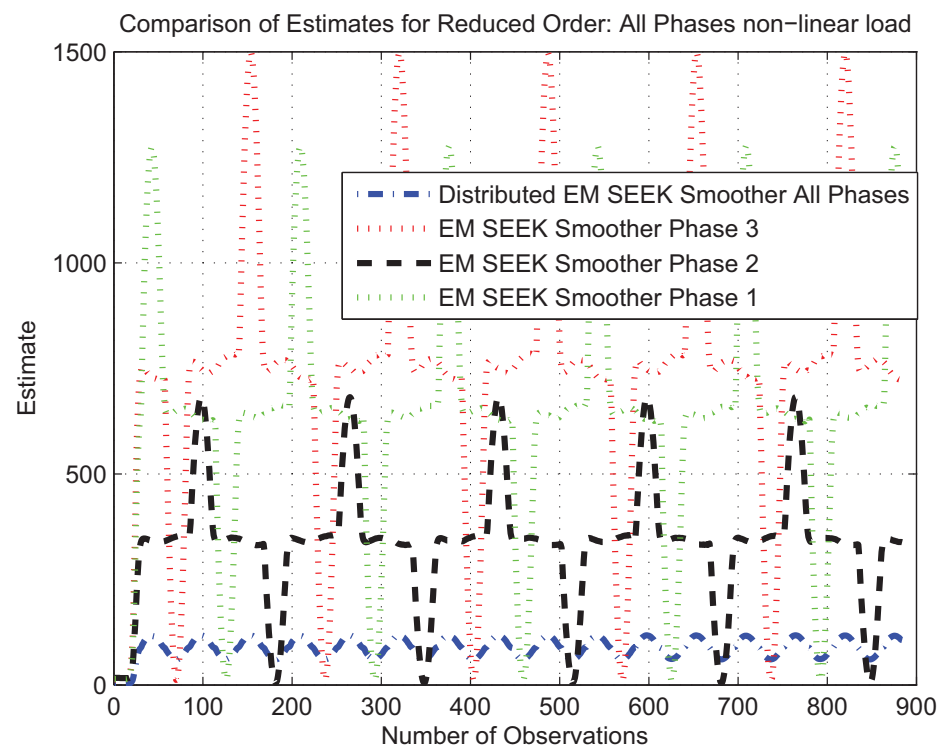

Figure 4.18: Estimates for various reduced-order smoothers: Nonlinear Load

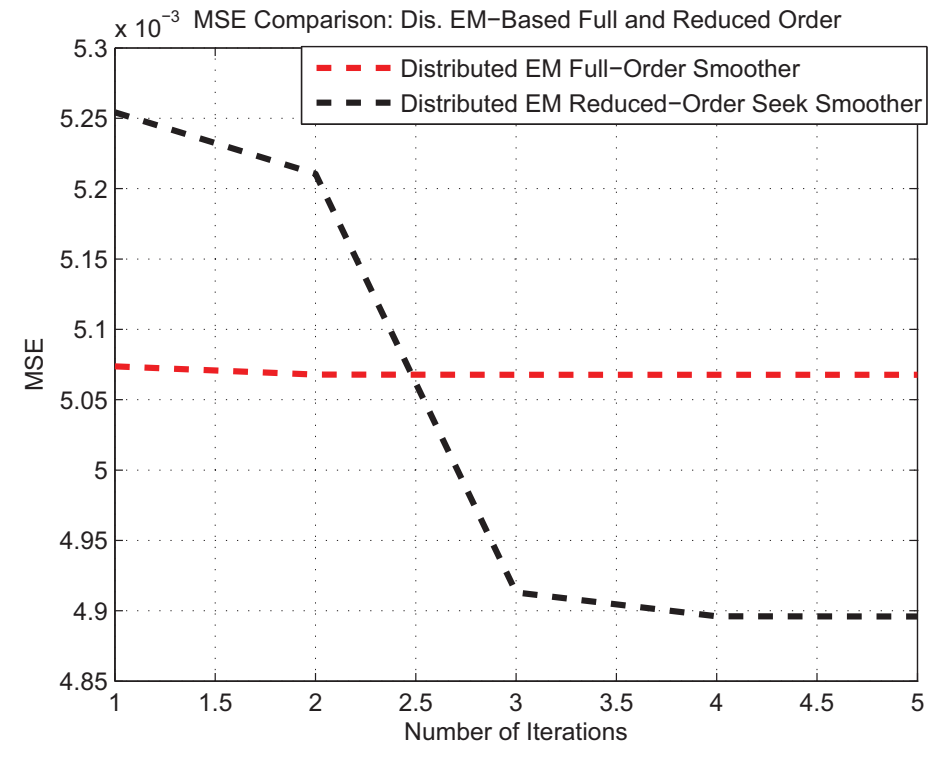

Figure 4.19: Mean Square Error: EM-Based full and reduced-order smoothers 


\section{Distributed Estimation Via IN-}

\section{FORMATION MATRIX APPROACH}

\subsection{AN OVERVIEW}

In this chapter, we have discussed distributed estimation via information matrix approach, where it is derived with various versions of information matrix filter. The estimation is derived on a distributed tracking system.

\subsection{INTRODUCTION}

Estimation is one of the precise solution in providing a strict surveillance system for an appropriate supervision. One of the methods to achieve such sort of estimation often requires a group of distributed sensors which provide information of the local targets. The classic work of Rao and Durrant-Whyte [381] presents an approach to decentralized Kalman filtering which accomplishes globally optimal performance in the case where all sensors can communicate with all other sensors. Other estimation methods can be a sensor-less approach [382][383], or a derivative-free filtering estimation [384], 
a least-squares-Kalman technique [386], a robot-based autonomous estimation and detection [385], $H_{\infty}$ filtering-based estimation made for stochastic incomplete measurements [387] etc.

During estimation, the problem of multi-target tracking utilizing information from multiple sensors employed has been in focus since last many years [388]-[396]. While achieving this approach, many fusion algorithms and filters were derived to combine local estimates local estimates [397][398][399][400] to prove better efficiency and effectiveness. For example, the state vectors can be fused using weighted covariance [406][407][408], information matrix [401], and covariance intersection [402][403]. The algorithms differ with the method they treat the covariance. As for the performance of different algorithms, [404] shows that the performance of weighted covariance algorithm is consistently worse as compared to the measurement fusion method. Moreover, it has been pointed out in [405] that results of weighted covariance algorithm are showing the behavior to be a maximum likelihood estimate. At the same time, Chang indicates that information matrix approach is optimal when the tracking systems are deterministic (i.e. process noise is zero) or when full-rate communication (i.e. two sensors exchange information each time when they receive new measurements and update their respective track files) is employed [405]. Covariance intersection avoids crosscovariance computation and its fusion result will be a consistent estimate, but its conservative estimates reduce performance [403]. However, covariance intersection is also being used for simultaneous localization and mapping to maintain the full correlation structure. 
In this chapter, we have derived distributed estimation with various versions of information matrix filter. The estimation is derived on a distributed tracking system. After achieving a distributed estimation with various versions, we have stemmed two methods for measurement fusion. The proposed scheme is then validated on a industrial utility boiler system, where different types of faults were introduced and were considered for the evaluation of the proposed scheme.

The remainder of this chapter is structured as follows. Problem formulation is described in Section II. The information-based covariance intersection filter is derived and discussed in Section III, followed by the information-based weighted covariance filter and Kalman-like particle filter derived and discussed in Section IV and Section V respectively. Measurement fusion algorithm is discussed in Section VI, followed by some evaluation and testing in Section VII. Finally some conclusion is made in Section VIII.

\subsection{PROBLEM Formulation}

Consider a distributed tracking system, as in [410] in which $N(N \geq 2)$ sensors are tracking the same target. The mathematical model describing target dynamic is assumed to be linear time invariant and of the form:

$$
x_{k+1}=F x_{k}+G v_{k}, k=0,1,2, \ldots
$$


where $x_{k} \in \Re^{n_{1}}$ is state vector of target at time $k$ and $F$ is state transition matrix, $v_{k} \in$ $\Re^{n_{2}}$ is zero mean white Gaussian process noise with known covariance $Q$, and $G$ is the input matrix. The target is tracked by $N$ sensors, where measurement model of sensor $j=1, \ldots, N$ is described by:

$$
z_{k}^{j}=H^{j} x_{k}+w_{k}^{j}
$$

where $w_{k}^{j} \in \Re^{n_{3}}$ is zero-mean white Gaussian measurement noise with covariance $\Re_{k}^{j}$.

It is assumed that local track estimates, $\hat{x}_{k \mid k}^{j}$ and $P_{k \mid k}^{j}$, where $j=1, \ldots, N$ are obtained by each sensor's information-based filter based on measurement sequence $Z_{k}^{j}=$ $\left\{z_{i}^{j}, i=1,2, \ldots, k\right\}$ and are optimal in the sense of minimum variance. At the end of each $n$ sampling interval, each sensor transmits its local estimate to fusion center where track association and fusion are performed. For fused estimate, there are two choices: either be sent back to sensor to improve local estimation performance or to store on fusion center. For the sake of simplicity, the dimension of the fused track and all local tracks are assumed to be the same. The distributed track fusion problem is to generate an "optimal" estimate $\hat{x}_{k \mid k}$ from all local track information, i.e. $\hat{x}_{k \mid k}^{j}$ and $P_{k \mid k}^{j}$, and prior information about local and fused estimation if possible [396]. The following sections work on the derived versions of information-based filters for the distributed tracking system. 


\subsection{COVARIANCE INTERSECTION}

According to the standard results of covariance intersection in [412], the covariance intersection at the sensor is:

$$
\begin{aligned}
\hat{x}_{k \mid k} & =P_{k \mid k}\left(\omega P_{k \mid k}^{i}{ }^{-1} \hat{x}_{k \mid k}^{i}+(1-\omega) P_{k \mid k}^{i}{ }^{-1} \hat{x}_{k \mid k}^{j}\right) \\
K_{1} & =\omega P_{k \mid k} P_{k \mid k}^{i}{ }^{-1} \\
K_{2} & =(1-\omega) P_{k \mid k} P_{k \mid k}^{j-1}
\end{aligned}
$$

where $K_{1}$ and $K_{2}$ are the gains and $\omega \in[0,1]$ and it manipulates the weights which are assigned to $\hat{x}_{k \mid k}^{i}$ and $\hat{x}_{k \mid k}^{j}$ respectively. The covariance of filtering error is given by:

$$
P_{k \mid k}=\left(\omega P_{k \mid k}^{i}{ }^{-1}+(1-\omega) P_{k \mid k}^{j-1}\right)^{-1}
$$

Or

$$
P_{k \mid k}^{-1}=\left(\omega P_{k \mid k}^{i}{ }^{-1}+(1-\omega) P_{k \mid k}^{j}{ }^{-1}\right)
$$

where $\omega=\left(K_{1} / P_{k \mid k}\right) \cdot P_{k \mid k}^{i}$ and $1-\omega=\left(K_{2} / P_{k \mid k}\right) \cdot P_{k \mid k}^{j}$, where $P_{k \mid k}^{i}$ and $P_{k \mid k}^{j}$ are error covariance matrices.

Thus substituting (5.4), (5.5), (5.7) into (5.3) yields

$$
P_{k \mid k}^{-1} x_{k \mid k}=\omega P_{k \mid k}^{i}{ }^{-1} x_{k \mid k}^{i}+(1-\omega) P_{k \mid k}^{j}{ }^{-1} x_{k \mid k}^{j}
$$


The main agenda is to bring two equations of inverse covariance and its product with the state from every covariance technique derived.

Remark 5.4.1 Different choices of $\omega$ can be used to optimize the update with respect to different performance criteria such as minimizing the trace or determinant of $P_{k \mid k}$.

\subsubsection{Information-BASED COVARiance InTERSECTION FiLter}

\section{ALGORITHM}

For the case of deriving information-based covariance intersection filter, the target dynamic model of (5.1) and (5.2) will be of the form:

$$
\begin{aligned}
x_{k+1} & =F x_{k}^{i}+F x_{k}^{j}+G \nu_{k} \\
z_{k}^{j} & =K_{1} x_{k}^{i}+K_{2} x_{k}^{j}+w_{k}
\end{aligned}
$$

The key idea of the information matrix filter is to identify the common information shared by estimates that are to be fused, and then removing the information or decorrelation is implemented. It will take into consideration the common information but not the common process noise. Under the assumption of no feedback, the estimation 
using information-based filter in the case of covariance intersection is as follows:

$$
\begin{aligned}
P_{k \mid k}^{-1} \hat{x}_{k \mid k} & =P_{k \mid k-n}^{-1} \hat{x}_{k \mid k-n}+\omega P_{k \mid k}^{i}{ }^{-1} \hat{x}_{k \mid k}^{i} \\
& -\omega P_{k \mid k-n}^{i}{ }^{-1} \hat{x}_{k \mid k-n}^{i}+(1-\omega) P_{k \mid k}^{j}{ }^{-1} \hat{x}_{k \mid k}^{j} \\
& -(1-\omega) P_{k \mid k-n}^{j}{ }^{-1} \hat{x}_{k \mid k n}^{j} \\
P_{k \mid k}^{-1} & =P_{k \mid k-n}^{-1}+\omega P_{k \mid k}^{i}{ }^{-1}-\omega P_{k \mid k-n}^{i}{ }^{-1}+(1-\omega) P_{k \mid k}^{j}{ }^{-1} \\
& -(1-\omega) P_{k \mid k-n}^{j}{ }^{-1}
\end{aligned}
$$

where the $n$ step fusion state prediction is:

$$
x_{k \mid k-n}=F x_{k}^{i}+F x_{k}^{j}
$$

The associated covariance is explained by the following theorem.

Theorem 5.1 Following [413], since $v_{k}$ is assumed to be $m \times 1$ zero-mean white noise process, and $x_{k}$ the $n \times 1$ so-called state vector, it can be easily seen from $x_{k+1}$ $=F x_{k}^{i}+F x_{k}^{j}+G v_{k}$ that covariance matrix of $x_{k}$ obeys the recursion,

$$
\Pi_{i+1}=F_{k} \Pi_{k}^{i} F_{k}^{*}+F_{k} \Pi_{k}^{j} F_{k}^{*}+G_{i} Q_{i} G_{i}^{*}
$$

where $\Pi_{k}^{i}=\mathbf{E} x_{k}^{i} x_{k}^{i *}$ and $\Pi_{k}^{j}=\mathbf{E} x_{k}^{j} x_{k}^{j^{*}}$.

Likewise, since $\hat{x}_{k \mid k-n}=F x_{k}^{i}+F x_{k}^{j}$, then it satisfies the recursion,

$$
\Sigma_{i+1}=F_{k}^{i} \Sigma_{k}^{i} F_{k}^{i^{*}}+F_{k}^{j} \Sigma_{k}^{j} F_{k}^{j^{*}}
$$


where $\Sigma_{k}^{i}=\mathbf{E} \hat{x}_{k \mid k-1}^{i} \hat{x}_{k \mid k-1}^{i^{*}}$ and $\Sigma_{k}^{j}=\mathbf{E} \hat{x}_{k \mid k-1}^{j} \hat{x}_{k \mid k-1}^{j^{*}}$ with initial condition $\Sigma_{0}=0$. Now the orthogonal decomposition $x_{i}=\hat{x}_{k \mid k-1}+$ with $\hat{x}_{i \mid i-1}$, shows that $\Pi_{i}=\Sigma_{k}^{i}+\Sigma_{k}^{j}+$ $P_{k \mid k-1}$. It is then immediate to conclude that $P_{k+1 \mid k}=\Sigma_{k+1}-\Sigma_{k+1}^{i}+\Sigma_{k+1}^{j}$ satisfies the recursion

$$
P_{k+1 \mid k}=F_{k}^{i} P_{k \mid k-1} F_{k}^{i^{*}}+G_{i} Q_{i} G_{i}^{*}
$$

As for the distributed tracking system, the communication network is considered to be large, therefore, the fused state estimate and associated covariance depends upon the local estimates as:

$$
\begin{aligned}
& \hat{x}_{k \mid k-n}^{i}+\hat{x}_{k \mid k-n}^{j}=\hat{x}_{k \mid k-n} \\
& P_{k \mid k-n}^{i}+P_{k \mid k-n}^{j}=P_{k \mid k-n}
\end{aligned}
$$

\subsubsection{INFORMATION-BASED COVARIANCE INTERSECTION FILTER: Complete Feedback Case}

For the case of complete feedback, closed form analytical solution of steady fused covariance of information-based covariance intersection filter with $N$ sensors is derived 
below. From (5.9) and (5.10), it is easy to show that the following two equations hold,

$$
\begin{aligned}
x_{k} & =F_{k}^{i} x_{k-n}+F_{k}^{j} x_{k-n}+\sum_{i=1}^{n} F^{n-i} G v_{k-n+i} \\
z_{k}^{j} & =K_{1} F^{i} x_{k-n}^{i}+K_{2}^{j} F^{j} x_{k-n}^{j}+w_{k-n}+K_{1} F^{i} G v_{k-n+i} \\
& +K_{2} F^{j} G v_{k-n+j}
\end{aligned}
$$

For the two local sensors in covariance intersection i.e. $i$ and $j$, it is possible to write

$$
x_{k \mid k}=\omega P_{k \mid k} P_{k \mid k}^{i}{ }^{-1} F x_{k \mid k}^{i}+(1-\omega) P_{k \mid k} P_{k \mid k}^{j}{ }^{-1} F x_{k \mid k}^{j}
$$

Using (5.21) and (5.17), we have

$$
\hat{x}_{k \mid k}=A_{n} x_{k \mid k}^{i}+B_{i} x_{k \mid k}^{j}
$$

where, $\forall i=1, \ldots, n$, we have $A_{0}=I, A_{i}=\omega A_{i-1} P_{k \mid k} P_{k \mid k}^{i}{ }^{-1} F, B_{i}=(1-\omega) A_{i-1} P_{k \mid k} P_{k \mid k}^{j}{ }^{-1} F$.

Under the assumption of complete feedback, (5.11) and (5.12) can be re-written as:

$$
\begin{aligned}
P_{k \mid k}^{-1} \hat{x}_{k \mid k} & =-(N-1) P_{k \mid k-n}^{-1} \hat{x}_{k \mid k-n}+\omega P_{k \mid k}^{i}{ }^{-1} \hat{x}_{k \mid k}^{i} \\
& +(1-\omega) P_{k \mid k}^{j}{ }^{-1} \hat{x}_{k \mid k}^{j} \\
P_{k \mid k}^{-1} & =-(N-1) P_{k \mid k-n}^{-1}+\omega P_{k \mid k}^{i-1} \\
& +(1-\omega) P_{k \mid k}^{j-1}
\end{aligned}
$$


To compute the steady state error covariance of fused state estimate, subtracting $P_{k \mid k}^{-1} x_{k}$, from both sides of (5.23), and substituting (5.22) yields

$$
\begin{aligned}
P_{k \mid k}^{-1}\left(\hat{x}_{k \mid k}-x_{k}\right) & =-P_{k \mid k}^{-1} x_{k}-(N-1) P_{k \mid k-n}^{-1} \hat{x}_{k \mid k-n} \\
& +\omega P_{k \mid k}^{i}{ }^{-1} \hat{x}_{k \mid k}^{i}+(1-\omega) P_{k \mid k}^{j}{ }^{-1} \hat{x}_{k \mid k}^{j} \\
& =-(N-1) P_{k \mid k-n}^{-1} F^{n}\left(\hat{x}_{k \mid k-n}-x_{k-n}\right) \\
& -P_{k \mid k}^{-1} x_{k}-(N-1) P_{k \mid k-n}^{-1} F^{n} x_{k-n} \\
& +P_{k \mid k}^{-1}\left[A_{n} x_{k \mid k}^{i}+B_{i} x_{k \mid k}^{j}\right]
\end{aligned}
$$

Through simple algebra manipulation and substituting (5.20) into (5.25) as:

$$
\begin{aligned}
P_{k \mid k}^{-1}\left(\hat{x}_{k \mid k}-x_{k}\right)= & \left\{-(N-1) P_{k \mid k-n}^{-1} F^{n}+P_{k \mid k}^{-1} A_{n}\right\} \\
\cdot & \left(\hat{x}_{k-n \mid k-n}-x_{k-n}\right)+P_{k \mid k}{ }^{-1} A_{n} \hat{x}_{k-n} \\
- & P_{k \mid k}^{-1} x_{k}-(N-1) P_{k \mid k-n}^{-1} F^{n} x_{k-n} \\
+ & P_{k \mid k}^{-1} B_{i} x_{k \mid k}^{j} \\
= & \left\{-(N-1) P_{k \mid k-n}^{-1} F^{n}+P_{k \mid k}^{-1} A_{n}\right\} \\
& \left(\hat{x}_{k-n \mid k-n}-x_{k-n}\right)+P_{k \mid k}{ }^{-1} A_{n} \hat{x}_{k-n} \\
- & (N-1) P_{k \mid k-n}^{-1} F^{n} x_{k-n} \\
+ & P_{k \mid k}^{-1} B_{i} w_{k-n+i}-P_{k \mid k}^{-1} x_{k} \\
+ & P_{k \mid k}^{-1} B_{i}\left(K_{1} F^{i} x_{k-n}^{i}+K_{2} F^{j} x_{k-n}^{j}\right) \\
+ & P_{k \mid k}^{-1} B_{i} \sum_{h=1}^{i}\left(K_{1}+K_{2}\right) \\
& F^{i-h} G v_{k-n+h} \\
&
\end{aligned}
$$


It has been proven in [394] that $A_{n}$ satisfies the following identity

$$
A_{n}=-\sum_{i=1}^{n} B_{i} K F^{\prime}+F^{n}
$$

Substituting (5.27) and (5.24) into (5.26), we have

$$
\begin{aligned}
P_{k \mid k}^{-1}\left(\hat{x}_{k \mid k}-x_{k}\right)= & \left\{-(N-1) P_{k \mid k-n}^{-1} F^{n}+P_{k \mid k}^{-1} A_{n}\right\} \\
\cdot & \left(\hat{x}_{k-n \mid k-n}-x_{k-n}\right)+P_{k \mid k}^{-1} A_{n} x_{k-n} \\
- & (N-1) P_{k \mid k-n}^{-1} F^{n} x_{k-n}+P_{k \mid k}^{-1} B_{i} w_{k-n+i} \\
- & P_{k \mid k}^{-1} x_{k}+P_{k \mid k}^{-1}\left(F^{n}-A_{n}\right) x_{k-n} \\
+ & P_{k \mid k}^{-1} B_{i} \sum_{h=1}^{i} F^{i-h} G v_{k-n+h} \\
= & \left\{-(N-1) P_{k \mid k-n}^{-1} F^{n}+P_{k \mid k}^{-1} A_{n}\right\} \\
& \left(\hat{x}_{k-n \mid k-n}-x_{k-n}\right)+P_{k \mid k}^{-1} B_{i} w_{k-n+i} \\
+ & \left(P_{k \mid k}^{-1} B_{i} \sum_{h=i}^{n}\left(K_{1}+K_{2}\right) F^{h-i}-P_{k \mid k}^{-1}\right. \\
& \left.F^{n-i}\right) G v_{k-n+i}
\end{aligned}
$$

Using (5.28), showing a Lyapunov form as follows

$$
\Omega_{x}=C_{f} \Omega_{x} C_{f}^{\prime}+\Omega_{f}
$$


where

$$
\begin{aligned}
C_{f} & =\lim _{k \rightarrow \infty} P_{k \mid k}\left(-(N-1) P_{k \mid k-n}^{-1} F^{n}+P_{k \mid k}^{i^{-1}} A_{n}^{i}+P_{k \mid k}^{j^{-1}} A_{n}^{j}\right), \\
\Omega_{f} & =W_{s}(k) R W_{s}(k)^{\prime}+V_{s}(k) G Q G^{\prime} V_{s}(k), \\
W_{s}(k) & =\lim _{k \rightarrow \infty} P_{k \mid k} P_{k \mid k}^{-1} B_{i}, \\
V_{s}(k) & =\lim _{k \rightarrow \infty} P_{k \mid k} P_{k \mid k}^{-1} B_{i} \sum_{h=1}^{n}\left(K_{1}+K_{2}\right) F^{h-i} \\
& -P_{k \mid k} P_{k \mid k}^{-1} F^{n-i}
\end{aligned}
$$

\subsubsection{INFORMATION-BASED COVARIANCE INTERSECTION FILTER:}

\section{Partial FeEdBack CASE}

In the case of partial feedback, (5.11) and (5.12) can be formulated as follows:

$$
\begin{aligned}
P_{k \mid k}^{-1} \hat{x}_{k \mid k} & =P_{k \mid k-n}^{-1} \hat{x}_{k \mid k-n}+\omega P_{k \mid k}^{i}{ }^{-1} \hat{x}_{k \mid k}^{i} \\
& -\omega P_{k \mid k-n}^{i}{ }^{-1} \hat{x}_{k \mid k-n}+(1-\omega) P_{k \mid k}^{j^{-1}} \hat{x}_{k \mid k}^{j} \\
& -(1-\omega) P_{k \mid k-n}^{j^{-1}} \hat{x}_{k \mid k-n} \\
P_{k \mid k}^{-1} & =P_{k \mid k-n}^{-1}+\omega P_{k \mid k}^{i^{-1}}-\omega P_{k \mid k-n}^{i^{-1}}+(1-\omega) P_{k \mid k}^{j^{-1}} \\
& -(1-\omega) P_{k \mid k-n}^{j^{-1}}
\end{aligned}
$$

Note that changing the value of $N$ does not alter the forms of (5.31) and (5.32) and only length of summation item need to be adjusted. Like the case of complete feedback, 
there is also a discrete Lyapunov equation,

$$
\Omega_{x}=C_{p} \Omega_{x} C_{p}^{\prime}+\Omega_{p}
$$

where

$$
\begin{aligned}
C_{p} & =\lim _{k \rightarrow \infty} P_{k \mid k}\left[P_{k \mid k}^{i^{-1}} A_{n}^{i}+P_{k \mid k}^{j^{-1}} A_{n}^{j}-P_{k \mid k-n}^{i^{-1}} F^{n}\right. \\
& \left.-P_{k \mid k-n}^{j^{-1}} F^{n}+P_{k \mid k-n}^{-1} F^{n}\right]
\end{aligned}
$$

with $\Omega_{p}$ has the same definition of $\Omega_{f}$ in (5.30).

\subsection{WeIghted COVARIANCE}

According to the standard results of covariance intersection in [412], the weighted covariance at the sensor is:

$$
\hat{x}_{k \mid k}=A_{k}^{i} \hat{x}_{k \mid k}^{i}+A_{k}^{j} \hat{x}_{k \mid k}^{j}
$$

where the weighted matrices of two local estimates are calculated as:

$$
\begin{aligned}
& A_{k}^{i}=\left(P_{k \mid k}^{j}-\Sigma_{k \mid k}^{j, i}\right)\left(P_{k \mid k}^{i}+P_{k \mid k}^{j}-\Sigma_{k \mid k}^{i j}-\Sigma_{k \mid k}^{j i}\right)^{-1} \\
& A_{k}^{j}=\left(P_{k \mid k}^{i}-\Sigma_{k \mid k}^{i, j}\right)\left(P_{k \mid k}^{i}+P_{k \mid k}^{j}-\Sigma_{k \mid k}^{i j}-\Sigma_{k \mid k}^{j i}\right)^{-1}
\end{aligned}
$$


And covariance of fused estimate is computed as:

$$
\begin{aligned}
P_{k \mid k}= & P_{k \mid k}^{j}-\left(P_{k \mid k}^{j}-\Sigma_{k \mid k}^{j, i}\right)\left(P_{k \mid k}^{i}+P_{k \mid k}^{j}-\Sigma_{k \mid k}^{i j}-\Sigma_{k \mid k}^{j i}\right)^{-1} \\
& \cdot\left(P_{k \mid k}^{j}-\Sigma_{k \mid k}^{j i}\right)^{T}
\end{aligned}
$$

Or

$$
\begin{aligned}
P_{k \mid k}^{-1}= & \left(P_{k \mid k}^{j}-\left(P_{k \mid k}^{j}-\Sigma_{k \mid k}^{j, i}\right)\left(P_{k \mid k}^{i}+P_{k \mid k}^{j}-\Sigma_{k \mid k}^{i j}-\Sigma_{k \mid k}^{j i}\right)^{-1}\right. \\
& \left.\cdot\left(P_{k \mid k}^{j}-\Sigma_{k \mid k}^{j i}\right)^{T}\right)^{-1}
\end{aligned}
$$

where $\Sigma_{1 \mid 1}^{i, j}=\left(I-K_{1}^{i} H_{1}^{i}\right) Q_{0}\left(I-K_{1}^{i} H_{1}^{i}\right)^{T}, \Sigma_{k \mid k}^{i, j}=\left(I-K_{k}^{i} H_{k}^{i}\right) F_{k-1} \sum_{k-1 \mid k-1}^{i, j} F_{k-1}^{T}(I-$ $\left.K_{k}^{i} H_{k}^{i}\right)^{T}+\left(I-K_{k}^{i} H_{k}^{i}\right) Q_{k-1}\left(I-K_{k}^{i} H_{k}^{i}\right)^{T}$, and $\Sigma_{k \mid k}^{j, i}=\left(\Sigma_{k \mid k}^{i, j}\right)^{T}$. Multiplying (5.39) with (5.35) gives:

$$
\begin{aligned}
P_{k \mid k}^{-1} \hat{x}_{k \mid k} & =\left(P_{k \mid k}^{j}-\left(P_{k \mid k}^{j}-\Sigma_{k \mid k}^{j, i}\right)\left(P_{k \mid k}^{i}+P_{k \mid k}^{j}-\Sigma_{k \mid k}^{i j}\right.\right. \\
& \left.\left.-\Sigma_{k \mid k}^{j i}\right)^{-1} \cdot\left(P_{k \mid k}^{j}-\Sigma_{k \mid k}^{j i}\right)^{T}\right)^{-1} \\
& \cdot\left(A_{k}^{i} \hat{x}_{k \mid k}^{i}+A_{k}^{j} \hat{x}_{k \mid k}^{j}\right)
\end{aligned}
$$




\subsubsection{Information-BAsed Weighted Covariance Filter Al-} GORITHM

For the case of deriving information-based weighted covariance filter, the target dynamic model of (5.1) and (5.2) will be of the form:

$$
\begin{aligned}
x_{k+1} & =F x_{k}+G w_{k} \\
z_{k} & =H^{i} x_{k}+H^{j} x_{k}+v^{i}+v^{j}
\end{aligned}
$$

The key idea of the information matrix filter is to identify the common information shared by estimates that are to be fused, and then removing the information or decorrelation is implemented. It will take into consideration the common information but not the common process noise. Under the assumption of no feedback, the estimation 
using information-based filter in the case of weighted covariance is as follows:

$$
\begin{aligned}
P_{k \mid k}^{-1} \hat{x}_{k \mid k} & =P_{k \mid k-n}^{-1} \hat{x}_{k \mid k-n}+\left(P_{k \mid k}^{j}-\left(P_{k \mid k}^{j}-\Sigma_{k \mid k}^{j, i}\right)\right. \\
& \cdot\left(P_{k \mid k}^{i}+P_{k \mid k}^{j}-\Sigma_{k \mid k}^{i j}-\Sigma_{k \mid k}^{j i}\right)^{-1}\left(P_{k \mid k}^{j}\right. \\
& \left.\left.-\Sigma_{k \mid k}^{j i}\right)^{T}\right)^{-1} \cdot\left(A_{k}^{i} \hat{x}_{k \mid k}^{i}+A_{k}^{j} \hat{x}_{k \mid k}^{j}\right)-\left(P_{k \mid k-n}^{j}\right. \\
& -\left(P_{k \mid k-n}^{j}-\Sigma_{k \mid k-n}^{j, i}\right) \cdot\left(P_{k \mid k-n}^{i}+P_{k \mid k-n}^{j}-\Sigma_{k \mid k-n}^{i j}\right. \\
& \left.\left.-\Sigma_{k \mid k-n}^{j i}\right)^{-1} \cdot\left(P_{k \mid k-n}^{j}-\Sigma_{k \mid k-n}^{j i}\right)^{T}\right)^{-1}\left(A_{k}^{i} \hat{x}_{k \mid k-n}^{i}\right. \\
& \left.+A_{k}^{j} \hat{x}_{k \mid k-n}^{j}\right) \\
& =P_{k \mid k-n}^{-1}+\left(P_{k \mid k}^{j}-\left(P_{k \mid k}^{j}-\Sigma_{k \mid k}^{j, i}\right) \cdot\left(P_{k \mid k}^{i}+P_{k \mid k}^{j}\right.\right. \\
P_{k \mid k}^{-1} & \left.\left.\Sigma_{k \mid k}^{i j}-\Sigma_{k \mid k}^{j i}\right)^{-1}\left(P_{k \mid k}^{j}-\Sigma_{k \mid k}^{j i}\right)^{T}\right)^{-1}-\left(P_{k \mid k-n}^{j}\right. \\
& -\left(P_{k \mid k-n}^{j}-\Sigma_{k \mid k-n}^{j, i}\right) \cdot\left(P_{k \mid k-n}^{i}+P_{k \mid k-n}^{j}-\Sigma_{k \mid k-n}^{i j}\right. \\
& \left.\left.-\Sigma_{k \mid k-n}^{j i}\right)^{-1} \cdot\left(P_{k \mid k-n}^{j}-\Sigma_{k \mid k-n}^{j i}\right)^{T}\right)^{-1}
\end{aligned}
$$

The $n$ step fusion state prediction and associated covariance from Theorem 5.1 is shown

as:

$$
\begin{aligned}
& \hat{x}_{k \mid k-n}=F^{i} \hat{x}_{k-n \mid k-n}+F^{j} \hat{x}_{k-n \mid k-n} \\
& P_{k+1 \mid k}=F_{k}^{i} P_{k \mid k-1} F_{k}^{i^{*}}+G_{i} Q_{i} G_{i}^{*}
\end{aligned}
$$


The fused state estimate and associated covariance depends upon the local estimates as:

$$
\begin{aligned}
& \hat{x}_{k \mid k-n}^{i}+\hat{x}_{k \mid k-n}^{j}=\hat{x}_{k \mid k-n} \\
& P_{k \mid k-n}^{i}+P_{k \mid k-n}^{j}=P_{k \mid k-n}
\end{aligned}
$$

\subsubsection{Information-Based Weighted Covariance Filter: Com- PLETE FEEDBACK CASE}

For the case of complete feedback, closed form analytical solution of steady fused covariance of information-based covariance intersection filter with $N$ sensors is derived below. From (5.41) and (5.42), it is easy to show that the following two equations hold,

$$
\begin{aligned}
x_{k} & =F_{k}^{i} x_{k-n}+F_{k}^{j} x_{k-n}+\sum_{i=1}^{n} F^{n-i} G v_{k-n+i} \\
z_{k} & =H^{i} F^{i} x_{k-n}+H^{j} F^{j} x_{k-n}+w_{k-n+i}^{i}+w_{k-n+j}^{j} \\
& +H^{i} F^{i} G v_{k-n+i}+H^{j} F^{j} G v_{k-n+j}
\end{aligned}
$$

For the local sensors, it is possible to write weighted covariance as:

$$
\begin{aligned}
\hat{x}_{k \mid k} & =P_{k \mid k}\left(P_{k \mid k}^{j} F \hat{x}_{k \mid k-n}+\left(P_{k \mid k}^{j}-\Sigma_{k \mid k}^{j i}\right)\left(P_{k \mid k}^{i}+P_{k \mid k}^{j}\right.\right. \\
& \left.\left.-\Sigma_{k \mid k}^{i j}-\Sigma_{k \mid k}^{j i}\right)^{-1}\left(P_{k \mid k}^{j}-\Sigma_{k \mid k}^{j i}\right)^{T}\right)^{-1} P_{k \mid k} \\
& \cdot\left(A_{k}^{i} F \hat{x}_{k \mid k}^{i}+A_{k}^{j} F \hat{x}_{k \mid k}^{j}\right)
\end{aligned}
$$


Using (5.51) and (5.49), we have

$$
\hat{x}_{k \mid k}=A_{n} P_{k \mid k} A_{k}^{i} F x_{k \mid k}^{i}+A_{n} P_{k \mid k} A_{k}^{j} F x_{k \mid k}^{j}
$$

where, $\forall i=1, \ldots, n$, we have $A_{0}=I, A_{i}=A_{i-1} P_{k \mid k}\left(P_{k \mid k}^{j} F \hat{x}_{k \mid k-n}+\left(P_{k \mid k}^{j}-\Sigma_{k \mid k}^{j i}\right)\left(P_{k \mid k}^{i}+\right.\right.$ $\left.\left.P_{k \mid k}^{j}-\Sigma_{k \mid k}^{i j}-\Sigma_{k \mid k}^{j i}\right)^{-1}\left(P_{k \mid k}^{j}-\Sigma_{k \mid k}^{j i}\right)^{T}\right)^{-1}$ Under the assumption of complete feedback, (5.43) and (5.44) can be re-written as:

$$
\begin{aligned}
P_{k \mid k}^{-1} \hat{x}_{k \mid k}= & -(N-1) P_{k \mid k-n}^{-1} \hat{x}_{k \mid k-n}+\left(P_{k \mid k}^{j}-\left(P_{k \mid k}^{j}-\Sigma_{k \mid k}^{j, i}\right)\right. \\
\cdot & \left.\left(P_{k \mid k}^{i}+P_{k \mid k}^{j}-\Sigma_{k \mid k}^{i j}-\Sigma_{k \mid k}^{j i}\right)^{-1} \cdot\left(P_{k \mid k}^{j}-\Sigma_{k \mid k}^{j i}\right)^{T}\right)^{-1} \\
\cdot & \left(A_{k}^{i} \hat{x}_{k \mid k}^{i}+A_{k}^{j} \hat{x}_{k \mid k}^{j}\right) \\
P_{k \mid k}^{-1}= & -(N-1) P_{k \mid k-n}^{-1}+\left(P_{k \mid k}^{j}-\left(P_{k \mid k}^{j}-\Sigma_{k \mid k}^{j, i}\right)\right. \\
\cdot & \left(P_{k \mid k}^{i}+P_{k \mid k}^{j}-\Sigma_{k \mid k}^{i j}-\Sigma_{k \mid k}^{j i}\right)^{-1} \\
\cdot & \left.\left(P_{k \mid k}^{j}-\Sigma_{k \mid k}^{j i}\right)^{T}\right)^{-1}
\end{aligned}
$$


To compute the steady state error covariance of fused state estimate, subtracting $P_{k \mid k}^{-1} x_{k}$ from both sides of (5.53) and substituting (5.52) yields

$$
\begin{aligned}
P_{k \mid k}^{-1}\left(\hat{x}_{k \mid k}-x_{k}\right) & =-P_{k \mid k}^{-1} x_{k}-(N-1) P_{k \mid k-n}^{-1} \hat{x}_{k \mid k-n} \\
& -(N-1) P_{k \mid k-n}^{-1} \hat{x}_{k \mid k-n}+\left(P_{k \mid k}^{j}-\left(P_{k \mid k}^{j}\right.\right. \\
& \left.-\Sigma_{k \mid k}^{j, i}\right) \cdot\left(P_{k \mid k}^{i}+P_{k \mid k}^{j}-\Sigma_{k \mid k}^{i j}-\Sigma_{k \mid k}^{j i}\right)^{-1} \\
& \left.\cdot\left(P_{k \mid k}^{j}-\Sigma_{k \mid k}^{j i}\right)^{T}\right)^{-1} \cdot\left(A_{k}^{i} \hat{x}_{k \mid k}^{i}+A_{k}^{j} \hat{x}_{k \mid k}^{j}\right) \\
& =-(N-1) P_{k \mid k-n}^{-1} F^{n}\left(\hat{x}_{k-n \mid k-n}-x_{k-n}\right) \\
& -P_{k \mid k}^{-1} x_{k}-(N-1) P_{k \mid k-n}^{-1} F^{n} x_{k-n} \\
& +P_{k \mid k}^{-1}\left(A_{n} P_{k \mid k} A_{k}^{i} F x_{k \mid k}^{i}\right. \\
& \left.+A_{n} P_{k \mid k} A_{k}^{j} F \hat{x}_{k \mid k}^{j}\right)
\end{aligned}
$$

Through simple algebra manipulations and substituting (5.50), we can re-write (5.55) as

$$
\begin{aligned}
P_{k \mid k}^{-1}\left(\hat{x}_{k \mid k}-x_{k}\right) & =\left(-(N-1) P_{k \mid k-n}^{-1} F^{n}+P_{k \mid k}^{-1} A_{n} P_{k \mid k} A_{k}^{i} F\right. \\
& \left.+P_{k \mid k}^{-1} A_{n} P_{k \mid k} A_{k}^{j} F\right) \cdot\left(\hat{x}_{k-n \mid k-n}-\hat{x}_{k \mid k}^{i}\right. \\
& \left.-\hat{x}_{k \mid k}^{j}\right)+P_{k \mid k}^{-1} A_{n} P_{k \mid k} A_{k}^{i} F \hat{x}_{k-n}^{i}+P_{k \mid k}^{-1} \\
& \cdot A_{n} P_{k \mid k} A_{k}^{j} F \hat{x}_{k \mid k-n}^{j}-P_{k \mid k}^{-1} x_{k} \\
& -(N-1) P_{k \mid k-n}^{-1} F^{n} x_{k-n}
\end{aligned}
$$


Using (5.56), showing a Lyapunov form as follows:

$$
\Omega_{x}=C_{f} \Omega_{x} C_{f}^{\prime}+\Omega_{f}
$$

where

$$
\begin{aligned}
C_{f} & =\lim _{k \rightarrow \infty} P_{k \mid k}\left(-(N-1) P_{k \mid k-n}^{-1} F^{n}+P_{k \mid k}^{-1} A_{n}\right. \\
& \left.\cdot P_{k \mid k} A_{k}^{i} F+P_{k \mid k}^{-1} A_{n} P_{k \mid k} A_{k}^{j} F\right) \\
\Omega_{f} & =W_{s}(k) R W_{s}(k)^{\prime}, \\
W_{s}(k) & =\lim _{k \rightarrow \infty} P_{k \mid k} P_{k \mid k}^{-1} A_{n} P_{k \mid k}\left(A_{k}^{i}+A_{k}^{j}\right)
\end{aligned}
$$




\subsubsection{Information-Based Weighted Covariance Filter: Par-}

\section{TIAL FEEDBACK CASE}

In the case of partial feedback, (5.43) and (5.44) can be formulated as follows:

$$
\begin{aligned}
P_{k \mid k}^{-1} \hat{x}_{k \mid k} & =P_{k \mid k-n}^{-1} \hat{x}_{k \mid k-n}+\left(P_{k \mid k}^{j}-\left(P_{k \mid k}^{j}-\Sigma_{k \mid k}^{j, i}\right)\right. \\
& \cdot\left(P_{k \mid k}^{i}+P_{k \mid k}^{j}-\Sigma_{k \mid k}^{i j}-\Sigma_{k \mid k}^{j i}\right)^{-1}\left(P_{k \mid k}^{j}\right. \\
& \left.\left.-\Sigma_{k \mid k}^{j i}\right)^{T}\right)^{-1} \cdot\left(A_{k}^{i} \hat{x}_{k \mid k}^{i}+A_{k}^{j} \hat{x}_{k \mid k}^{j}\right)-\left(P_{k \mid k-n}^{j}\right. \\
& -\left(P_{k \mid k-n}^{j}-\Sigma_{k \mid k-n}^{j, i}\right) \cdot\left(P_{k \mid k-n}^{i}+P_{k \mid k-n}^{j}-\Sigma_{k \mid k-n}^{i j}\right. \\
& \left.\left.-\Sigma_{k \mid k-n}^{j i}\right)^{-1} \cdot\left(P_{k \mid k-n}^{j}-\Sigma_{k \mid k-n}^{j i}\right)^{T}\right)^{-1}\left(A_{k}^{i} \hat{x}_{k \mid k-n}\right. \\
& \left.+A_{k}^{j} \hat{x}_{k \mid k-n}\right) \\
& =P_{k \mid k-n}^{-1}+\left(P_{k \mid k}^{j}-\left(P_{k \mid k}^{j}-\Sigma_{k \mid k}^{j, i}\right) \cdot\left(P_{k \mid k}^{i}+P_{k \mid k}^{j}\right.\right. \\
P_{k \mid k}^{-1} & \left.\left.\Sigma_{k \mid k}^{i j}-\Sigma_{k \mid k}^{j i}\right)^{-1}\left(P_{k \mid k}^{j}-\Sigma_{k \mid k}^{j i}\right)^{T}\right)^{-1} \\
& -\left(P_{k \mid k-n}^{j}-\left(P_{k \mid k-n}^{j}-\Sigma_{k \mid k-n}^{i j}\right.\right. \\
& \left.\left.-\Sigma_{k \mid k-n}^{j i}\right)^{-1} \cdot\left(P_{k \mid k-n}^{j}-\Sigma_{k \mid k-n}^{j i}\right)^{T}\right)^{-1}
\end{aligned}
$$

Note that changing the value of $N$ does not alter the forms of (5.59) and (5.60) and only length of summation item need to be adjusted. Like the case of complete feedback, there is also a discrete Lyapunov equation,

$$
\Omega_{x}=C_{p} \Omega_{x} C_{p}^{\prime}+\Omega_{p}
$$


where

$$
\begin{aligned}
C_{p} & =\lim _{k \rightarrow \infty} P_{k \mid k}\left[P_{k \mid k}^{i^{-1}} A_{n}^{i} P_{k \mid k} A_{k}^{i} F+P_{k \mid k}^{j^{-1}} A_{n}^{j} P_{k \mid k} A_{k}^{j} F\right. \\
& \left.-P_{k \mid k-n}^{i^{-1}} F^{n}-P_{k \mid k-n}^{j^{-1}} F^{n}+P_{k \mid k-n}^{-1} F^{n}\right]
\end{aligned}
$$

with $\Omega_{p}$ has the same definition of $\Omega_{f}$ in (5.58).

\subsection{Kalman-Like Particle Filter}

In this Section, we will derive information-based Kalman-like particle filter, where the simple Kalman-like particle filter is expressed in [414]. A question arises here that why Kalman-like particle filter has been preferred on a basic Kalman filter? The justification for the approach w.r.t filter is given in [414], moreover, it is preferred here on the basic Kalman filter because of the following. (See Fig. 5.2 for the comparison of estimates of a basic Kalman filter and Kalman-like particle filter. See Fig. 5.2 where it can be seen, how the mean square error is reduced in less number of iterations for particle

filter as compared to a regular Kalman filter): According to the standards results of Kalman-like particle filter in [414], the Kalman-like particle filter at sensor is:

$$
\begin{aligned}
\hat{x}_{k \mid k} & =\hat{x}_{k \mid k-1}+\frac{P_{k} H_{k}^{T}}{H_{k} P_{k} H_{k}^{T}+\sigma_{v}^{2}}\left(y_{k}-H_{k} \hat{x}_{k \mid k-1}\right) \\
& =\left(I-\frac{P_{k} H_{k}^{T}}{H_{k} P_{k} H_{k}^{T}+\sigma_{v}^{2}} H_{k}\right) \hat{x}_{k \mid k-1}+ \\
& +\frac{P_{k} H_{k}^{T}}{H_{k} P_{k} H_{k}^{T}+\sigma_{v}^{2}} y_{k}
\end{aligned}
$$




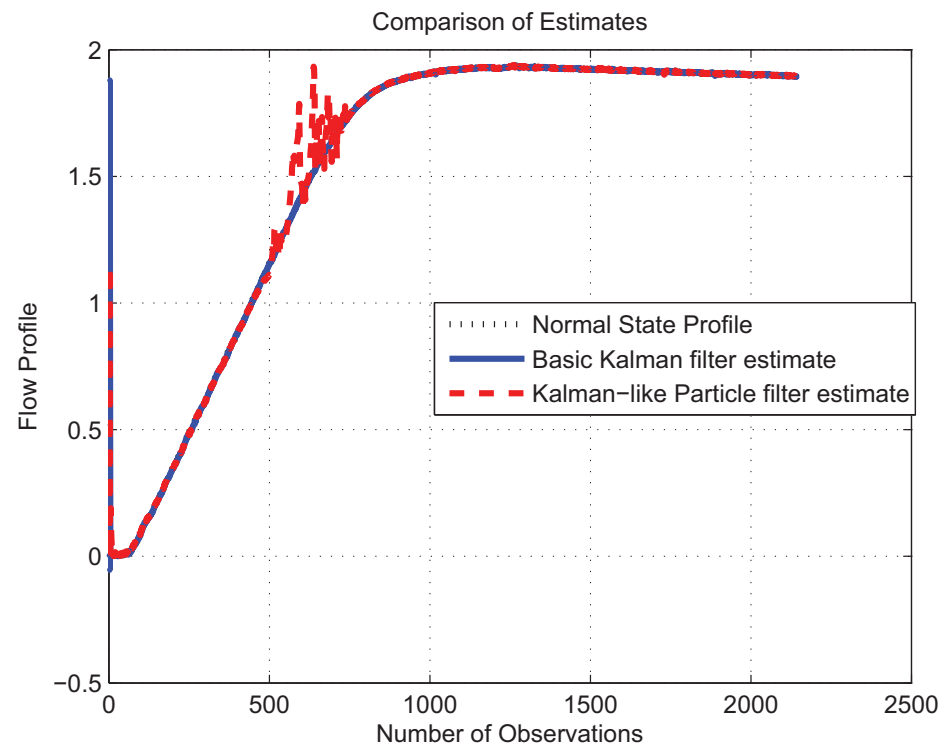

Figure 5.1: Estimates of Kalman-like particle and Basic Kalman filter

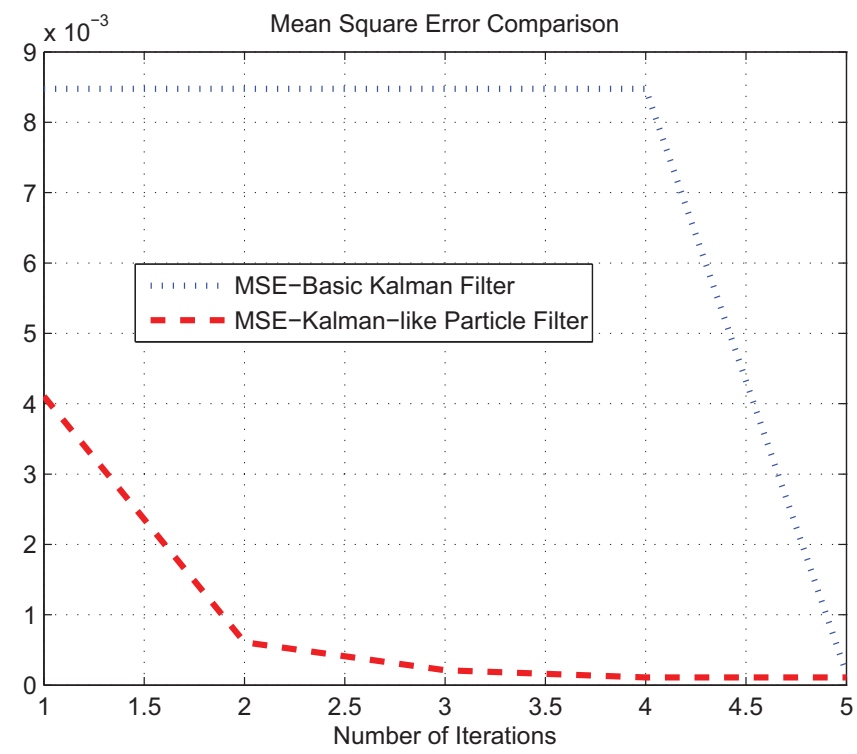

Figure 5.2: Mean Square Error: Kalman-like particle and basic Kalman filter 
with covariance of filtering error given by

$$
\begin{aligned}
P_{k \mid k} & =\left(I-\frac{P_{k} H_{k}^{T}}{H_{k} P_{k} H_{k}^{T}+\sigma_{v}^{2}} H_{k}\right) P_{k \mid k-1} \\
P_{k \mid k-1}^{-1} & =P_{k \mid k}^{-1}\left(I-\frac{P_{k} H_{k}^{T}}{H_{k} P_{k} H_{k}^{T}+\sigma_{v}^{2}} H_{k}\right)
\end{aligned}
$$

or

$$
P_{k \mid k}^{-1}=P_{k \mid k-1}^{-1}+P_{k \mid k}^{-1} \frac{P_{k} H_{k}^{T} H_{k}}{H_{k} P_{k} H_{k}^{T}+\sigma_{v}^{2}}
$$

Thus substituting (5.64) into (5.63) yields

$$
\begin{aligned}
P_{k \mid k}^{-1} \hat{x}_{k \mid k}= & P_{k \mid k-1}^{-1} \hat{x}_{k \mid k-1}+P_{k \mid k}^{-1} \\
& \cdot\left(\frac{P_{k} H_{k}^{T} H_{k}}{H_{k} P_{k} H_{k}^{T}+\sigma_{v}^{2}}\right) \hat{x}_{k \mid k}
\end{aligned}
$$

\subsubsection{Information-BASEd Kalman-Like PARticle Filter AL- GORITHM}

The key idea of the information matrix filter is to identify the common information shared by estimates that are to be fused, and then removing the information or decorrelation is implemented. It will take into consideration the common information but not the common process noise. Under the assumption of no feedback, the estimation 
using information-based filter in the case of Kalman-like particle filter is as follows:

$$
\begin{aligned}
P_{k \mid k}^{-1} \hat{x}_{k \mid k}= & P_{k \mid k-1}^{-1} \hat{x}_{k \mid k-1}+P_{k \mid k}^{j^{-1}} \\
& \cdot\left(\frac{P_{k}^{j} H_{k}^{j^{T}} H_{k}^{j}}{H_{k}^{j} P_{k}^{j} H_{k}^{j^{T}}+\sigma_{v}^{2}}\right) \hat{x}_{k \mid k}^{j} \\
& -P_{k \mid k-n}^{j^{-1}}\left(\frac{P_{k}^{j} H_{k}^{j^{T}} H_{k}^{j}}{H_{k}^{j} P_{k}^{j} H_{k}^{j^{T}}+\sigma_{v}^{2}}\right) \hat{x}_{k \mid k-n}^{j} \\
P_{k \mid k}^{-1} & =P_{k \mid k-1}^{-1}+P_{k \mid k}^{j^{-1}} \\
& \cdot\left(\frac{P_{k}^{j} H_{k}^{j^{T}} H_{k}^{j}}{H_{k}^{j} P_{k}^{j} H_{k}^{j^{T}}+\sigma_{v}^{2}}\right) \\
& -P_{k \mid k-n}^{j^{-1}}\left(\frac{P_{k}^{j} H_{k}^{j^{T}} H_{k}^{j}}{H_{k}^{j} P_{k}^{j} H_{k}^{j^{T}}+\sigma_{v}^{2}}\right)
\end{aligned}
$$

The $n$ step fusion state prediction and associated covariance from Theorem 5.1 is shown

as:

$$
\begin{aligned}
& x_{k \mid k-n}=F^{n} \hat{x}_{k-n \mid k-n} \\
& P_{k \mid k-n}=F^{n} P_{k-n \mid k-n} F^{n *}+F^{n-i} G Q G^{*} F^{n-i^{*}}
\end{aligned}
$$

where the $n$ step fusion state prediction and associated covariance is written as:

$$
\begin{aligned}
& \hat{x}_{k \mid k-n}^{j}=\hat{x}_{k \mid k-n} \\
& P_{k \mid k-n}^{j}=P_{k \mid k-n}
\end{aligned}
$$




\subsubsection{Information-Based Kalman-Like PARTiCle Filter: CoM- PLETE FEEDBACK CASE}

For the case of complete feedback, closed form analytical solution of steady fused covariance of information-based Kalman-like particle filter with $N$ sensors is derived below. From (5.1) and (5.2), it is easy to show that the following two equations hold,

$$
\begin{aligned}
x_{k} & =F_{k}^{i} x_{k-n}+F^{n-i} G v_{k-n+i} \\
z_{k-n+i}^{j} & =H^{j} F^{j} x_{k-n}+w_{k-n+i}^{j} \\
& +\sum_{h=1}^{i} H^{j} F^{i-h} G v_{k-n+h}
\end{aligned}
$$

For the two local sensor in Kalman-like particle filter, it is possible to write as:

$$
\begin{aligned}
\hat{x}_{k \mid k}^{j} & =P_{k \mid k} P_{k \mid k}^{j^{-1}} F \hat{x}_{k \mid k-1}^{j}+P_{k \mid k}^{j} P_{k \mid k}^{j^{-1}} \\
& \frac{P_{k}^{j} H_{k}^{j^{T}} H_{k}^{j}}{H_{k}^{j} P_{k}^{j} H_{k}^{j}+\sigma_{v}^{2}} \hat{x}_{k \mid k}
\end{aligned}
$$

Utilizing (5.71) and (5.75), we have

$$
\hat{x}_{k \mid k}^{j}=A_{n}^{j} \hat{x}_{k-n \mid k-n}+\sum_{i=1}^{n} B_{i}^{j} \hat{x}_{k \mid k}
$$

where, $\forall i=1, \ldots, n$, we have $A_{0}^{j}=I, A_{i}^{j}=P_{k-i+1 \mid k-i+1} P_{k-i+1 \mid k-i+1}^{j^{-1}} F, B^{j}=A_{i-1}^{j}$ $P_{k-i+1 \mid k-i+1}^{j} P_{k-i+1 \mid k-i+1}^{j^{-1}}\left(P_{k}^{j} H_{k}^{j^{T}} H_{k}^{j} /\left(H_{k}^{j} P_{k}^{j} H_{k}^{j}+\sigma_{v}^{2}\right)\right) F$. 
Under the assumption of complete feedback, (5.67) and (5.68) can be re-written as:

$$
\begin{aligned}
P_{k \mid k}^{-1} \hat{x}_{k \mid k} & =-(N-1) P_{k \mid k-n}^{-1} \hat{x}_{k \mid k-n} \\
& +\sum_{j=1}^{N} P_{k \mid k}^{j^{-1}} \frac{P_{k} H_{k}^{T} H_{k}}{H_{k} P_{k} H_{k}^{T}+\sigma_{v}^{2}} \hat{x}_{k \mid k}^{j} \\
P_{k \mid k}^{-1} & =-(N-1) P_{k \mid k-n}^{-1} \\
& +\sum_{j=1}^{N} P_{k \mid k}^{j^{-1}} \frac{P_{k} H_{k}^{T} H_{k}}{H_{k} P_{k} H_{k}^{T}+\sigma_{v}^{2}}
\end{aligned}
$$

To compute the steady state error covariance of fused state estimate, subtracting $P_{k \mid k}^{-1} x_{k}$ from both sides of (5.78) and substituting (5.76) yields

$$
\begin{aligned}
P_{k \mid k}^{-1}\left(\hat{x}_{k \mid k}-x_{k}\right) & =-P_{k \mid k}^{-1} x_{k}-(N-1) P_{k \mid k-n}^{-1} \hat{x}_{k \mid k-n} \\
& +\sum_{j=1}^{N} P_{k \mid k}^{j-1} \frac{P_{k} H_{k}^{T} H_{k}}{H_{k} P_{k} H_{k}^{T}+\sigma_{v}^{2}} \hat{x}_{k \mid k}^{j} \\
& =-(N-1) P_{k \mid k-n}^{-1} F^{n}\left(\hat{x}_{k \mid k-n}-x_{k-n}\right) \\
& -P_{k \mid k}^{-1} x_{k}-(N-1) P_{k \mid k-n}^{-1} F^{n} x_{k-n} \\
& +\sum_{j=1}^{N} P_{k \mid k}^{j^{-1}} \frac{P_{k} H_{k}^{T} H_{k}}{H_{k} P_{k} H_{k}^{T}+\sigma_{v}^{2}} \\
& \cdot\left[A_{n}^{j} \hat{x}_{k-n \mid k-n}+\sum_{i=1}^{n} B_{i}^{j} x_{k \mid k}\right]
\end{aligned}
$$

Through simple algebra manipulation and substituting (5.75), we can re-write (5.79) 
as:

$$
\begin{aligned}
P_{k \mid k}^{-1}\left(\hat{x}_{k \mid k}-x_{k}\right)= & \left(-(N-1) P_{k \mid k-n}^{-1} F^{n}\right. \\
+ & \left.\sum_{j=1}^{N} P_{k \mid k}^{j^{-1}}\left(\frac{P_{k} H_{k}^{T} H_{k}}{H_{k} P_{k} H_{k}^{T}+\sigma_{v}^{2}}\right) A_{n}^{j}\right) \\
& \cdot\left(\hat{x}_{k-n \mid k-n}-x_{k-n}\right)+\sum_{j=1}^{N} P_{k \mid k}^{j^{-1}} \\
& \left(\frac{P_{k} H_{k}^{T} H_{k}}{H_{k} P_{k} H_{k}^{T}+\sigma_{v}^{2}}\right) A_{n}^{j} x_{k-n}-P_{k \mid k}^{-1} x_{k} \\
& -(N-1) P_{k \mid k-n}^{-1} F^{n} x_{k-n} \\
& +\left(\sum_{j=1}^{N} P_{k \mid k}^{j-1} \sum_{i=1}^{n} B_{i}^{j} x_{k \mid k}\right)
\end{aligned}
$$

Using (5.80), showing a Lyapunov form as follows:

$$
\Omega_{x}=C_{f} \Omega_{x} C_{f}^{\prime}+\Omega_{f}
$$

where

$$
\begin{aligned}
C_{f} & =\lim _{k \rightarrow \infty} P_{k \mid k}\left(-(N-1) P_{k \mid k-n}^{-1} F^{n}+\sum_{j=1}^{n} P_{k \mid k}^{-1}\right. \\
& \left.\cdot \frac{P_{k} H_{k}^{T} H_{k}}{H_{k} P_{k} H_{k}^{T}+\sigma_{v}^{2}} A_{n}^{j}\right), \\
\Omega_{f} & =\sum_{j=1}^{N} \sum_{k=1}^{n} W_{s}^{j}(k) R^{j} W_{s}^{j}(k)^{\prime}, \\
W_{s}^{j}(k) & =\lim _{k \rightarrow \infty} P_{k \mid k} P_{k \mid k}^{j^{-1}} B_{i}^{j}
\end{aligned}
$$




\subsubsection{INFORMATION-BASED KALMAN-LIKE PARTICLE FILTER: PAR-}

\section{TIAL FEEDBACK CASE}

In the case of partial feedback, (5.67) and (5.68) can be formulated as follows:

$$
\begin{aligned}
P_{k \mid k}^{-1} \hat{x}_{k \mid k} & =P_{k \mid k-n}^{-1} \hat{x}_{k \mid k-n} \\
& +\sum_{j=1}^{N} P_{k \mid k}^{j^{-1}} \frac{P_{k} H_{k}^{T} H_{k}}{H_{k} P_{k} H_{k}^{T}+\sigma_{v}^{2}} \hat{x}_{k \mid k}^{j} \\
& -P_{k \mid k-n}^{j^{-1}} \frac{P_{k}^{j} H_{k}^{j^{T}} H_{k}^{j}}{H_{k}^{j} P_{k}^{j} H_{k}^{j^{T}}+\sigma_{v}^{2}} \hat{x}_{k \mid k-n}^{j} \\
P_{k \mid k}^{-1} & =P_{k \mid k-n}^{-1}+\sum_{j=1}^{N} P_{k \mid k}^{j^{-1}}\left(\frac{P_{k} H_{k}^{T} H_{k}}{H_{k} P_{k} H_{k}^{T}+\sigma_{v}^{2}}\right) \\
& -P_{k \mid k-n}^{j^{-1}}\left(\frac{P_{k}^{j} H_{k}^{j^{T}} H_{k}^{j}}{H_{k}^{j} P_{k}^{j} H_{k}^{j^{T}}+\sigma_{v}^{2}}\right)
\end{aligned}
$$

Note that changing the value of $N$ does not alter the forms of (5.83) and (5.84) and only length of summation item need to be adjusted. Like the case of complete feedback, there is also a discrete Lyapunov equation,

$$
\Omega_{x}=C_{p} \Omega_{x} C_{p}^{\prime}+\Omega_{p}
$$

where

$$
\begin{aligned}
C_{p} & =\lim _{k \rightarrow \infty} P_{k \mid k}\left[\sum_{j=1}^{n}\left(P_{k \mid k}^{-1} \cdot \frac{P_{k} H_{k}^{T} H_{k}}{H_{k} P_{k} H_{k}^{T}+\sigma_{v}^{2}} A_{n}^{j}-P_{k \mid k-n}^{j^{-1}} F^{n}\right)\right. \\
& \left.+P_{k \mid k-n}^{-1} F^{n}\right]
\end{aligned}
$$


with $\Omega_{p}$ has the same definition of $\Omega_{f}$ in (5.82).

\subsection{Measurement Fusion Algorithm}

The information captured in each of the information-based filter cases are designed for a distributed structure. The idea is taken from the fusion methods in [411].

Suppose there is $X$ number of sensors. For every measurement coming from these sensors that is received in fusion center, there is a corresponding estimation based solely on these individual sensors. The information can be structured as estimated information or prior estimated information in the following two ways which are measurement fusion method and state-vector fusion method as shown in the Fig. 5.3 and 5.4 respectively.

Measurement Fusion Method The measurement fusion method integrates the sensor measurement information by augmenting the observation vector as follows:

$$
\begin{aligned}
& y(k)=y^{(m f)}(k)=\left[\begin{array}{lll}
y_{1}(k) & \ldots & y_{N}(k)
\end{array}\right]^{T} \\
& C(k)=C^{(m f)}(k)=\left[\begin{array}{llll}
C_{1}(k) \ldots C_{N}(k) & ]^{T}
\end{array}\right. \\
& R(k)=R^{(m f)}(k)=\operatorname{diag}\left[R_{1}(k) \ldots R_{N}(k)\right]
\end{aligned}
$$

where the superscript $m f$ stands for the measurement fusion. 


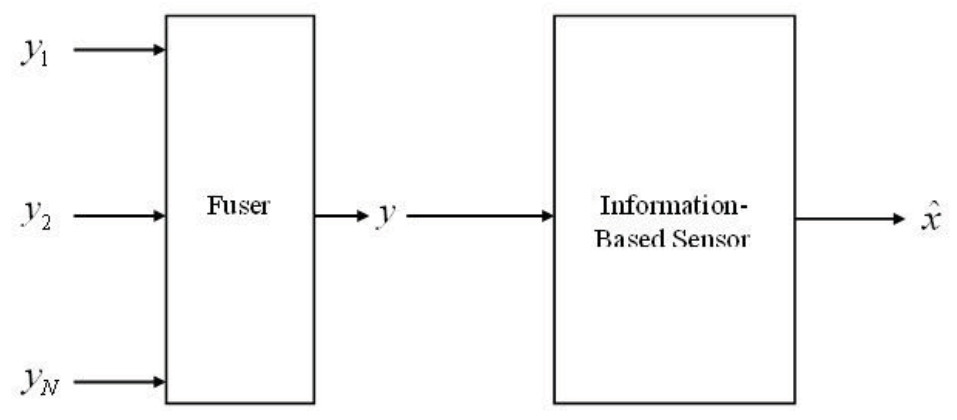

Figure 5.3: Measurement fusion employed for information-based sensor

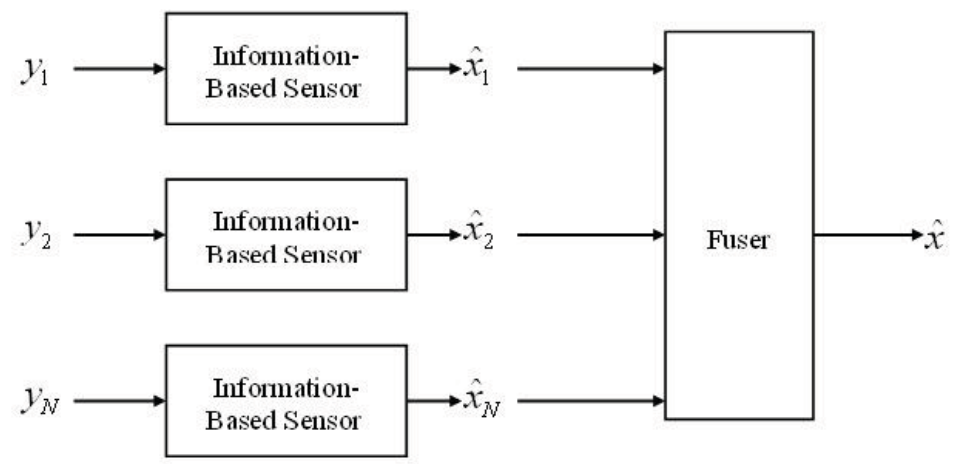

Figure 5.4: State vector fusion employed for information-based sensor 
Sate-vector Fusion Method The state-vector fusion method obtains the fused measurement information by weighted observation as follows:

$$
\begin{gathered}
y(k)=y^{(s f)}(k)=\left[\sum_{j=1}^{N} R_{j}^{-1}(k)\right]^{-1} \sum_{j=1}^{N} R_{j}^{-1}(k) y_{j}(k) \\
C(k)=C^{(s f)}(k)=\left[\sum_{j=1}^{N} R_{j}^{-1}(k)\right]^{-1} \sum_{j=1}^{N} R_{j}^{-1}(k) C_{j}(k) \\
R(k)=R^{(s f)}(k)=\left[\sum_{j=1}^{N} R_{j}^{-1}(k)\right]^{-1}
\end{gathered}
$$

where the superscript $s f$ stands for state-vector fusion.

\subsection{ON FUnCTIONAL EQUIVALENCE OF TWO MEASURE- MENT FUSION METHODS}

Comparing (5.87)-(5.89) with (5.90)-(5.92), we note that the treatment in the measurement fusion schemes is quite different. With reference to [411], we will show here that their exists a functional equivalence between the two methods.

Theorem 5.2 If the $N$ sensors used for data fusion with different and independent noise characteristics, have identical measurement matrices, i.e. $C_{1}(k)=C_{2}(k)=\ldots$ $=C_{N}(k)$, then the measurement fusion method is functionally equivalent to the statevector fusion. 
Proof. The following formula in linear algebra will be used to cope with the inversion of matrices:

$$
\begin{aligned}
{\left[\begin{array}{cc}
A_{1} & A_{2} \\
A_{3} & A_{4}
\end{array}\right]^{-1} } & =\left[\begin{array}{cc}
B_{1} & B_{2} \\
B_{3} & B_{4}
\end{array}\right] \\
\left(A+H B H^{t}\right)^{-1} & =A^{-1}-A^{-1} H\left(B^{-1}\right. \\
& \left.+H^{T} A^{-1} H\right)^{-1} H^{T} A^{-1}
\end{aligned}
$$

where $B_{1}=\left(A_{1}-A_{2} A_{4}^{-1} A_{3}\right)^{-1}, B_{2}=-B_{1} A_{2} A_{4}^{-1}, B_{3}=-A_{4}^{-1} A_{3} B_{1}$, and $B_{4}=$ $A_{4}^{-1}+A_{4}^{-1} A_{3} B_{1} A_{2} A_{4}^{-1}$. If the information-based covariance intersection filter is used, in order to demonstrate the functional equivalence of the two measurement fusion methods, we only need to check whether the terms $\left(K_{1}+K_{2}\right) C_{k}$ and $\left(K_{1}+K_{2}\right)(k) y(k)$ in measurement fusion method are functionally equivalent to those in state-vector fusion method. Alternatively, if the information filter is used, then we need to check the functional equivalence between terms $C^{T}(k) R^{-1}(k) C(k)$ and $C^{T}(k) R^{-1}(k) y(k)$ in both methods.

Consider the case when the information-based covariance intersection filter is applied, and $\left(K_{1}+K_{2}\right)^{(m f)}$ is:

$$
\begin{aligned}
&\left(K_{1}+\quad K_{2} \quad\right)^{(m f)}(k)= \\
& \omega P^{(m f)}(k \mid k-1)\left(C^{(s f)}\right)^{T}\left(C(k) P^{i}(k \mid k-1) C(k)\right. \\
&+\quadR(k))^{-1}+(1-\omega) P^{(m f)}(k \mid k-1)\left(C^{(s f)}\right)^{T} \\
&\left(C(k) P^{j}(k \mid k-1) C(k)+R(k)\right)^{-1}
\end{aligned}
$$


where $\Xi_{i}^{(m f)}=\left(C(k) P^{i}(k \mid k-1) C(k)+R(k)\right)^{-1}$ and $\Xi_{j}^{(m f)}=\left(C(k) P^{j}(k \mid k-1) C(k)+\right.$ $R(k))^{-1}$.

$$
\begin{aligned}
\left(K_{1}+K_{2} \quad\right)^{(m f)}(k)= & \\
& \omega P^{(m f)}(k \mid k-1)\left(C^{(s f)}\right)^{T} \\
\cdot & {\left[\begin{array}{cc}
R_{1}+\Xi_{i}^{(m f)} & \Xi_{i}^{(m f)} \\
\Xi_{i}^{(m f)} & R_{2}+\Xi_{i}^{(m f)}
\end{array}\right]^{-1} } \\
+ & (1-\omega) P^{m f}(k \mid k-1)\left(C^{(s f)}\right)^{T} \\
\cdot & {\left[\begin{array}{cc}
R_{1}+\Xi_{j}^{(m f)} & \Xi_{j}^{(m f)} \\
\Xi_{j}^{(m f)} & R_{2}+\Xi_{j}^{(m f)}
\end{array}\right]^{-1} }
\end{aligned}
$$




$$
\begin{aligned}
& \left(K_{1}+K_{2}\right)^{(m f)}(k)= \\
& \omega P^{(m f)}(k \mid k-1)(C)^{T}\left[\left(R_{2}+\Xi_{i}^{(m f)}\right)^{-1} \times R_{2}\right. \\
& \overbrace{\left[R_{1}+\Xi_{i}^{(m f)}-\Xi_{i}^{m f}\left(R_{2}\right.\right.}^{B_{1}}+\Xi_{i}^{(m f)})^{(m f)}]^{-1}, \\
& \times\left(_{\left(R_{2}+\Xi_{i}^{(m f)}\right.}^{A_{4}}\right)^{-1}-(\overbrace{R_{2}+\Xi_{i}^{(m f)}}^{A_{4}})^{-1} \\
& \times \overbrace{R_{2}}^{A_{3}}[\overbrace{R_{1}+\Xi_{i}^{(m f)}-\Xi_{i}^{(m f)}\left(R_{2}+\Xi_{i}^{(m f)}\right)^{-1} \Xi_{i}^{(m f)}}^{B_{1}}]^{-1} \\
& \times \overbrace{\Xi_{i}^{(m f)}}^{A_{2}} \overbrace{\left(R_{2}+\Xi_{i}^{(m f)}\right.}^{A_{4}})^{-1}+(1-\omega) P^{(m f)}(k \mid k-1) C^{T} \\
& \text {. } \quad\left[\left(R_{2}+\Xi_{j}^{(m f)}\right)^{-1} \times R_{2}[\overbrace{R_{1}+\Xi_{j}^{(m f)}}^{B_{1}}]-\Xi_{j}^{(m f)}\left(R_{2}\right.\right. \\
& \left.\left.+\Xi_{j}^{(m f)}\right)^{-1} \Xi_{j}^{(m f)}\right]^{-1} \times(\overbrace{R_{2}+\Xi_{j}^{(m f)}}^{A_{4}})^{-1} \\
& -\overbrace{\left(R_{2}+\Xi_{j}^{(m f)}\right.}^{A_{4}})^{-1} \\
& \times \overbrace{R_{2}}^{A_{3}}[\overbrace{R_{1}+\Xi_{j}^{(m f)}-\Xi_{i}^{(m f)}\left(R_{2}+\Xi_{j}^{(m f)}\right)^{-1} \Xi_{j}^{(m f)}}^{B_{1}}]^{-1} \\
& \times \overbrace{\Xi_{j}^{(m f)}}^{A_{2}} \overbrace{\left(R_{2}+\Xi_{j}^{(m f)}\right.}^{A_{4}})^{-1}
\end{aligned}
$$

where as proved in [411],

$$
\begin{aligned}
& \left(R_{2}+\Xi_{i}^{(m f)}\right)^{-1} R_{2}\left[R_{1}+\Xi^{(m f)}\right. \\
- & \left.\Xi_{i}^{(m f)}\left(R_{2}+\Xi_{i}^{(m f)}\right)^{-1} \Xi_{i}^{(m f)}\right]^{-1} \\
= & {\left[\Xi_{i}^{(m f)}+R_{1}\left(R_{1}+R_{2}\right)^{-1} R_{2}\right]^{-1} R_{2}\left(R_{1}+R_{2}\right)^{-1} }
\end{aligned}
$$


and

$$
\begin{aligned}
& \left(R_{2}+\Xi_{i}^{(m f)}\right)^{-1}-\left(R_{2}+\Xi_{i}^{(m f)}\right)^{-1} R_{2} \times\left[R_{1}+\Xi_{i}^{(m f)}-\Xi^{(m f)}\right. \\
\times & \left.\left(R_{2}+\Xi_{i}^{(m f)}\right)^{-1} \Xi_{i}^{(m f)}\right]^{-1} \Xi_{i}^{(m f)}\left(R_{2}+\Xi_{i}^{(m f)}\right)^{-1} \\
= & {\left[\Xi_{i}^{(m f)}+R_{1}\left(R_{1}+R_{2}\right)^{-1} R_{2}\right]^{-1} R_{1}\left(R_{1}+R_{2}\right)^{-1} }
\end{aligned}
$$

likewise for $\Xi_{j}^{(m f)}$ from equation (5.98) and (5.99). Based on (5.97)-(5.99), we have

$$
\begin{aligned}
\left(K_{1}+\quad K_{2} \quad\right)^{(m f)}(k)= & \\
& \omega P^{(m f)}(k \mid k-1) C^{T} \times\left[C P^{i^{(m f)}}(k \mid k-1) C^{T}\right. \\
+ & \left.R_{1}\left(R_{1}+R_{2}\right)^{-1} R_{2}\right]^{-1} \times\left[R_{2}\left(R_{1}+R_{2}\right)^{-1},\right. \\
& \left.R_{1}\left(R_{1}+R_{2}\right)^{-1}\right]+(1-\omega) P^{(m f)}(k \mid k-1) C^{T} \\
\times & {\left[C P^{j^{(m f)}}(k \mid k-1) C^{T}\right.} \\
+ & \left.R_{1}\left(R_{1}+R_{2}\right)^{-1} R_{2}\right]^{-1} \times\left[R_{2}\left(R_{1}+R_{2}\right)^{-1},\right. \\
& \left.R_{1}\left(R_{1}+R_{2}\right)^{-1}\right]
\end{aligned}
$$




$$
\begin{aligned}
&\left(K_{1}+\quad K_{2} \quad\right)^{(m f)}(k) C^{(m f)}(k)= \\
& \omega P^{(m f)}(k \mid k-1) C^{T} \times\left[C P^{i^{(m f)}}(k \mid k-1)\right. \\
&\left.\quad C^{T}+R_{1}\left(R_{1}+R_{2}\right)^{-1} R_{2}\right]^{-1} C \\
&+\quad(1-\omega) P^{(m f)}(k \mid k-1) C^{T} \\
& \times {\left[C P^{j^{(m f)}}(k \mid k-1) C^{T}\right.} \\
&+\left.R_{1}\left(R_{1}+R_{2}\right)^{-1} R_{2}\right]^{-1} C
\end{aligned}
$$

$$
\begin{aligned}
&\left(K_{1}+\quad K_{2} \quad\right)^{(m f)}(k) y^{(m f)}(k)= \\
& \omega P^{(m f)}(k \mid k-1) C^{T} \times\left[C P^{i^{(m f)}}(k \mid k-1)\right. \\
& \cdot \quad\left.C^{T}+R_{1}\left(R_{1}+R_{2}\right)^{-1} R_{2}\right]^{-1} \\
& \times \quad {\left[R_{2}\left(R_{1}+R_{2}\right)^{-1}\right.} \\
&\left.\quad y_{1}(t)+R_{1}\left(R_{1}+R_{2}\right)^{-1} y_{2}(t)\right]+(1-\omega) \\
& \quad P^{(m f)}(k \mid k-1) C^{T} \times\left[C P^{j^{(m f)}}(k \mid k-1) C^{T}\right. \\
&+\left.R_{1}\left(R_{1}+R_{2}\right)^{-1} R_{2}\right]^{-1} \times\left[R_{2}\left(R_{1}+R_{2}\right)^{-1}\right. \\
&\left.y_{1}(t)+R_{1}\left(R_{1}+R_{2}\right)^{-1} y_{2}(t)\right]
\end{aligned}
$$

If $C_{1}=C_{2}=C$, then $C^{(I I)}=C$, and we obtain the Kalman gain in state-vector method 
as follows:

$$
\begin{aligned}
&\left(K_{1}+\quad K_{2} \quad{ }^{(s f)}(k)=\right. \\
& \omega P^{(s f)}(k \mid k-1) C^{T} \times\left[C P^{i^{(s f)}}(k \mid k-1) C^{T}\right. \\
&+\left.R_{1}\left(R_{1}+R_{2}\right)^{-1} R_{2}\right]^{-1}+(1-\omega) P^{(s f)}(k \mid k-1) C^{T} \\
& \times \quad\left[C P^{j^{(s f)}}(k \mid k-1) C^{T}\right. \\
&\left.+\quad R_{1}\left(R_{1}+R_{2}\right)^{-1} R_{2}\right]^{-1}
\end{aligned}
$$


and we can derive the terms $K^{(s f)}(k) C^{(s f)}(k)$ and $K^{(s f)}(k) y^{(s f)}(k)$ :

$$
\begin{array}{cl}
\left(K_{1}+\quad K_{2} \quad{ }^{(s f)}(k) C^{(s f)}(k)=\right. \\
& \omega P^{(s f)}(k \mid k-1) C^{T} \times\left[C P^{i^{(s f)}}(k \mid k-1)\right. \\
\cdot & \left.C^{T}+R_{1}\left(R_{1}+R_{2}\right)^{-1} R_{2}\right]^{-1} C+(1-\omega) \\
\cdot & P^{(s f)}(k \mid k-1) C^{T} \times\left[C P^{j^{(s f)}}(k \mid k-1) C^{T}\right. \\
+ & \left.R_{1}\left(R_{1}+R_{2}\right)^{-1} R_{2}\right]^{-1} C \\
K_{2} & )^{(s f)}(k) y^{(s f)}(k)= \\
& \omega P^{(s f)}(k \mid k-1) C^{T} \times\left[C P^{i^{(s f)}}(k \mid k-1) C^{T}\right. \\
+ & \left.R_{1}\left(R_{1}+R_{2}\right)^{-1} R_{2}\right]^{-1} \times\left[R_{2}\left(R_{1}+R_{2}\right)^{-1}\right. \\
& \left.y_{1}(t)+R_{1}\left(R_{1}+R_{2}\right)^{-1} y_{2}(t)\right]+(1-\omega) \\
& P^{(s f)}(k \mid k-1) C^{T} \times\left[C P^{j^{(s f)}}(k \mid k-1) C^{T}\right. \\
+ & \left.R_{1}\left(R_{1}+R_{2}\right)^{-1} R_{2}\right]^{-1} \times\left[R_{2}\left(R_{1}+R_{2}\right)^{-1}\right. \\
& \left.y_{1}(t)+R_{1}\left(R_{1}+R_{2}\right)^{-1} y_{2}(t)\right]
\end{array}
$$

Note that (5.101) and (5.104) are in the same form and that (5.102) and (5.105) are also in the same form. Therefore, with the same initial conditions, i.e., $P^{(m f)}(0 \mid 0)$ $=P^{(s f)}(0 \mid 0)$ and $\hat{x}^{(m f)}(0 \mid 0)=\hat{x}^{(s f)}(0 \mid 0)$, the Kalman filters based on the observation information generated by (5.87-5.89) and (5.90-5.92), irrespectively, will result in the same state estimate $\hat{x}(k \mid k)$. This means that the two measurement fusion methods are functionally equivalent in the sensor-to-sensor case. 
Now, consider the case when the information filter is applied. From (5.87)(5.92), it is easy to prove the following equalities:

$$
\begin{aligned}
& {\left[C^{(m f)}(k)\right]^{T}\left[R^{(m f)}(k)\right]^{-1} C^{(m f)}(k) } \\
= & \sum_{j=1}^{N} C_{j}^{T} R_{j}^{-1} C_{j} \\
& {\left[C^{(m f)}(k)\right]^{T}\left[R^{(m f)}(k)\right]^{-1} y^{(m f)}(k) } \\
= & \sum_{j=1}^{N} C_{j}^{T} R_{j}^{-1} y_{j} \\
& {\left[C^{(s f)}(k)\right]^{T}\left[R^{(s f)}(k)\right]^{-1} C^{(s f)}(k)=\left[\left(\sum_{j=1}^{N} R_{j}^{-1}\right)^{-1}\right.} \\
\times & \left.\sum_{j=1}^{N} R_{j}^{-1} C_{j}\right]^{T} \sum_{j=1}^{N} R_{j}^{-1} C_{j} \\
& {\left[C^{(s f)}(k)\right]^{T}\left[R^{(s f)}(k)\right]^{-1} y^{(s f)}(k)=\left[\left(\sum_{j=1}^{N} R_{j}^{-1}\right)^{-1}\right.} \\
\times & \left.\sum_{j=1}^{N} R_{j}^{-1} C_{j}\right]^{T} \sum_{j=1}^{N} R_{j}^{-1} y_{j}
\end{aligned}
$$

If $C_{j}=C, j=1,2, \ldots, N$, then we have

$$
\begin{aligned}
& {\left[C^{(m f)}(k)\right]^{T}\left[R^{(m f)}(k)\right]^{-1} C^{(m f)}(k) } \\
= & {\left[C^{(s f)}(k)\right]^{T}\left[R^{(s f)}(k)\right]^{-1} C^{(s f)}(k) } \\
& {\left[C^{(m f)}(k)\right]^{T}\left[R^{(m f)}(k)\right]^{-1} y^{(m f)}(k) } \\
= & {\left[C^{(s f)}(k)\right]^{T}\left[R^{(s f)}(k)\right]^{-1} y^{(s f)}(k) }
\end{aligned}
$$

Remark 5.8.1 The functional equivalence is proved here with considering the gain $K$ as the center of existence for all the calculations, which can be the case for information- 
based weighted covariance filter too, but not for information-based Kalman-like particle filter where the gain $K$ is not present.

\subsection{Evaluation And Testing}

\subsubsection{UTILITY BOILER}

The evaluation and testing has been made on an industrial utility boiler [415]. In the system, the principal input variables are $u_{1}$, feedwater flow rate $(\mathrm{kg} / \mathrm{s}) ; u_{2}$, fuel flow rate $(\mathrm{kg} / \mathrm{s})$; and $u_{3}$, attemperator spray flow rate $(\mathrm{kg} / \mathrm{s})$, the states are $x_{1}$, fluid density, $x_{2}$, drum pressure, $x_{3}$, water flow input, $x_{4}$, fuel flow input, $x_{5}$, spray flow input. The principal output variables are $y_{1}$, drum level $(\mathrm{m}) ; y_{2}$, drum pressure $k P a$; and $y_{3}$, steam temperature $C^{0}$. The schematic diagram of the utility boiler can be seen in Fig. 5.5.

\section{Fault model for utility boiler}

Fault model for the utility boiler is being developed. The mathematical model of the faulty utility boiler can be given as follows where fault of steam pressure are there in state 4 (fuel flow input) and 5 (spray flow input) respectively (See Eqns. (5.112)(5.116)).

In the utility boiler, the steam temperature must be kept at a certain level to avoid overheating of the super-heaters. By applying a step to the water flow input (state 3), steam temperature increases and the steam temperature dynamics behaves like a fist order system. Applying a step to the fuel flow input (state 4), the steam temperature increases and the system behaves like a second order system. Applying a step to the 


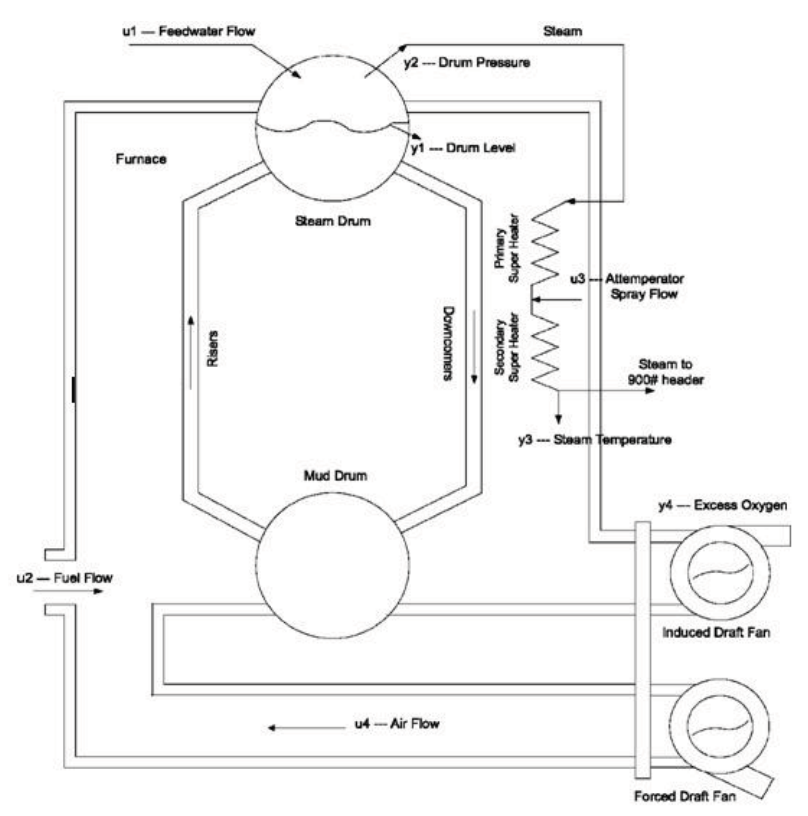

Figure 5.5: Schematic Diagram of an Industrial Utility Boiler

spray flow input (state 5), steam temperature decreases and the system behaves like a first order system. Then, a third order system is selected for the steam temperature model. Steam pressure is added there in state 4 and 5 resulting in a more uncontrolled non-linear system. Following [415] and the proposed fault scenarios, the fault model 
of the system can be described as:

$$
\begin{aligned}
& \dot{x}_{1}(t)=\frac{u_{1}-0.03 \sqrt{x_{2}^{2}-(6306)^{2}}}{155.1411} \\
& \dot{x}_{2}(t)=\left(-1.8506 \times 10^{-7} x_{2}-0.0024\right) \sqrt{x_{2}^{2}-(6306)^{2}} \\
& -0.0404 u_{1}+3.025 u_{2} \\
& \dot{x}_{3}(t)=-0.0211 \sqrt{x_{2}^{2}-(6306)^{2}}+x_{4}-0.0010967 u_{1} \\
& +0.0475 u_{2}+3.1846 u_{3} \\
& \dot{x}_{4}(t)=0.0015 \sqrt{x_{2}^{2}-(6306)^{2}}+x_{5}-0.001 u_{1} \\
& +0.32 u_{2}-2.9461 u_{3} \\
& +\left(a_{s t p r}\right) \sqrt{x_{2}^{2}-(6306)^{2}} \\
& \dot{x}_{5}(t)=-1.278 \times 10^{-3} \sqrt{x_{2}^{2}-(6306)^{2}} \\
& -0.00025831 x_{3}-0.29747 x_{4} \\
& -0.8787621548 x_{5}-0.00082 u_{1}-0.2652778 \\
& u_{2}+2.491 u_{3} \\
& +\left(a_{s t p r}\right) \sqrt{x_{2}^{2}-(6306)^{2}}
\end{aligned}
$$

\subsubsection{EVAluation OF RESUlts}

In what follows, we present simulation results for the proposed information-based versions of filters. The simulations have been performed on the utility boiler system where the faults due to steam pressure have been introduced in state 4 and 5 respectively. Firstly, the data generated from the simulation of the plant has been initialized and the 
parameters have been being optimized which comprises of the pre-processing and normalization of the data. The comparison of results for the distributed estimation, and normal estimation with different feedbacks generated from faults, and the basic profile of that particular state has been compared. Moreover, same pattern of comparison has been followed for all the versions of information-based filters.

\section{Information-Based Covariance Intersection filter}

The information-based covariance intersection filter has been simulated here for the utility boiler steam pressure fault of state 4 . Simulations have been made for the estimate of each case using state-vector fusion method. In the simulation, comparison of various profiles have been made i.e. profile of normal fault-free state, estimate of normal fault-free state, estimate of faulty state, distributed estimate based on state-vector fusion for different feedback strategies. The comparison of profiles mentioned above for complete feedback, partial feedback and no feedback profile can be seen in Fig. 5.6-5.8 respectively. Moreover, the one on one full comparison for all the feedback strategies can be seen in Fig. 5.9. It can be seen that here in case of information-based covariance intersection, the complete feedback case is performing better than the partial and no feedback case.

\section{Information-Based Weighted Covariance filter}

The information-based weighted covariance filter has been simulated here for the utility boiler steam pressure fault of state 4 . Simulations have been made for the estimate of each case using state-vector fusion method. In the simulation, comparison of various 
profiles have been made i.e. profile of normal fault-free state, estimate of normal faultfree state, estimate of faulty state, distributed estimate based on state-vector fusion for different feedback strategies. The comparison of profiles mentioned above for complete feedback and partial feedback profile can be seen in Fig. 5.10 and 5.11 respectively. Moreover, the one on one full comparison for all the feedback strategies can be seen in Fig. 5.12. It can be seen that here in case of information-based weighted covariance, the no feedback case is performing better than the partial feedback, and complete feedback has the lowest performance.

\section{Information-Based Kalman-like Particle filter}

The information-based Kalman-like particle filter has been simulated here for the utility boiler steam pressure fault of state 4 . Simulations have been made for the estimate of each case using state-vector fusion method. In the simulation, comparison of various profiles have been made i.e. profile of normal fault-free state, estimate of normal faultfree state, estimate of faulty state, distributed estimate based on state-vector fusion for different feedback strategies. The comparison of profiles mentioned above for complete feedback and partial feedback profile can be seen in Fig. 5.13 and 5.14 respectively. Moreover, the one on one full comparison for all the feedback strategies can be seen in Fig. 5.15. It can be seen that here in case of information-based Kalman-like particle filter, the partial feedback case is performing better than the complete feedback, and no feedback has the lowest performance. Also, a profile comparison for the measurement fusion method can be seen in Fig. 5.16 for a complete feedback case. 
Table 5.1: MSE Comparison for All Information-Based Filters*

\begin{tabular}{l|c|c|c|}
\hline FILTER & COMPLETE FB & PARTIAL FB & NO FB \\
\hline \hline CI & 6.424 & 8.2759 & 8.411 \\
\hline WC & $1.031 \times 10^{-3}$ & $1.0273 \times 10^{-3}$ & $1.0275 \times 10^{-3}$ \\
\hline KLPF & 0.565 & 0.703 & 0.6223 \\
\hline
\end{tabular}

\section{Mean Square Error Comparison}

In this section, we have made a comparison of the all versions of information-based filters with complete, partial and no feedback respectively. It can be seen from Table 5.1 that how the feedback versions are performing differently for a particular case of information-based filter. The mean square error value of complete feedback is the minimum in the case of information-based covariance intersection filter and Kalman-like particle filter respectively, whereas partial feedback is performing well in the case of information-based weighted covariance filter.

$11 *$ The table is showing the comparison of all the versions of information-based filters, where MSE stands for mean square error, FB stands for feedback, CI stands for covariance intersection, WC stands for weighted covariance and KLPF stands for Kalman-like Particle filter 


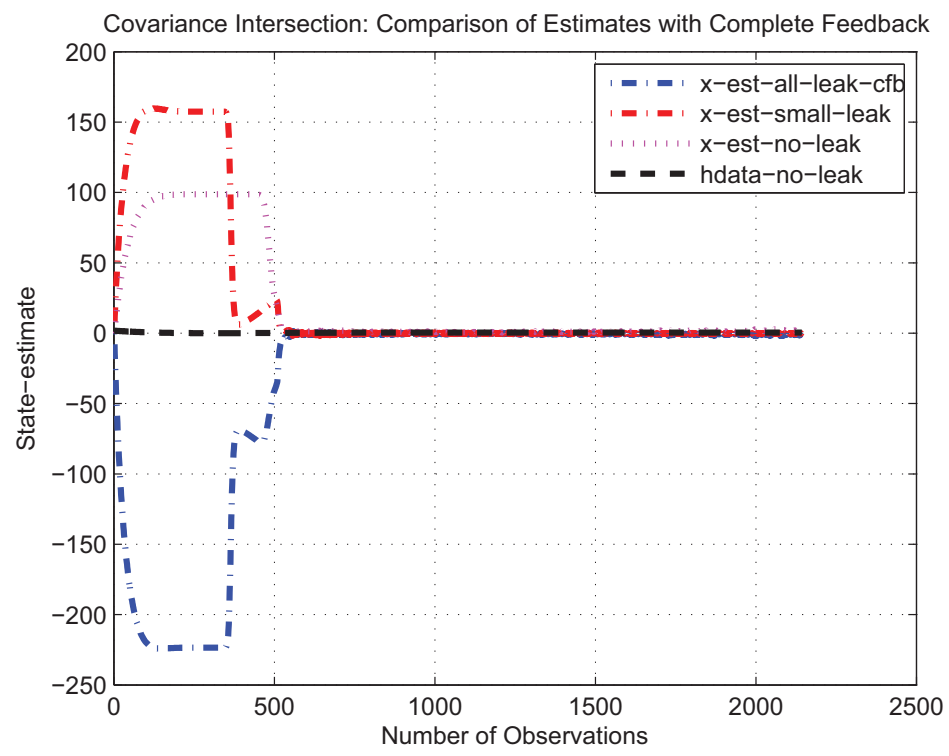

Figure 5.6: Covariance Intersection: Complete Feedback Comparison

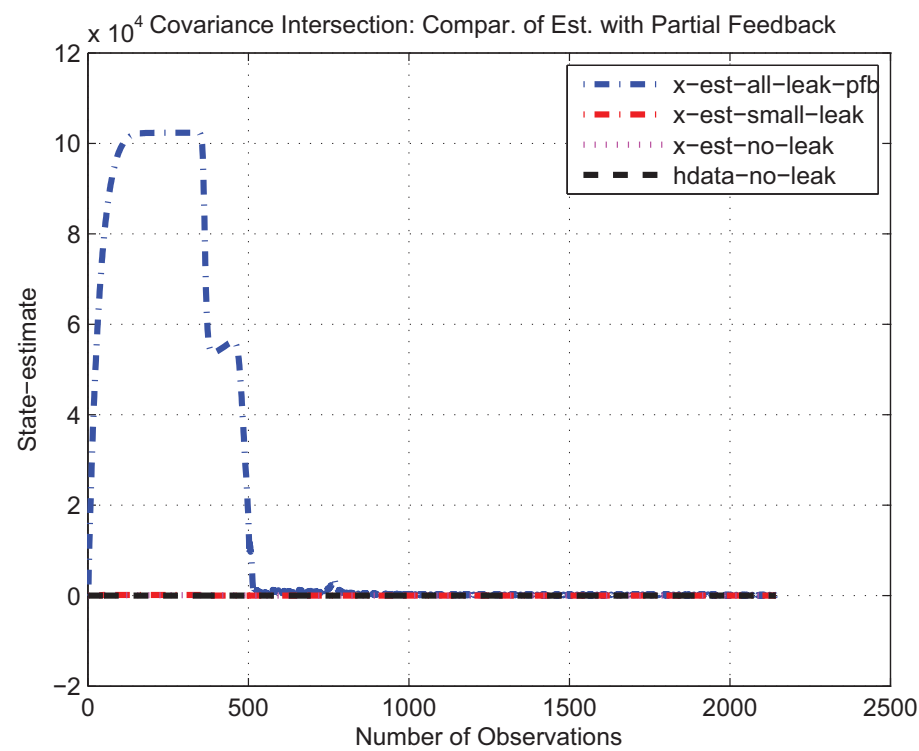

Figure 5.7: Covariance Intersection: Partial Feedback Comparison 


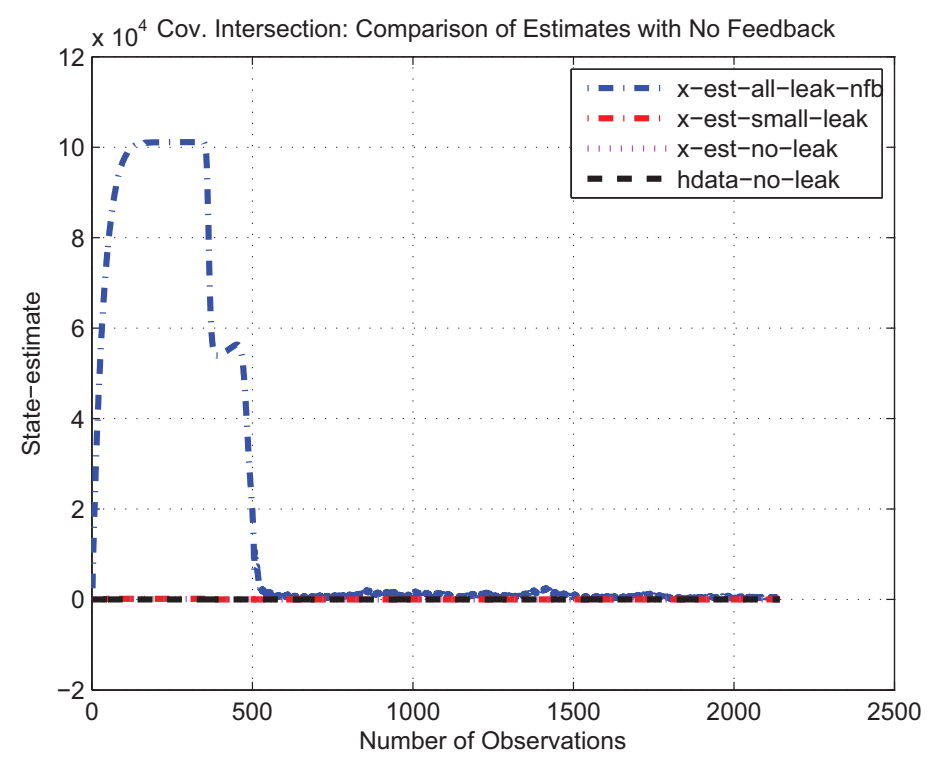

Figure 5.8: Covariance Intersection: No Feedback Comparison

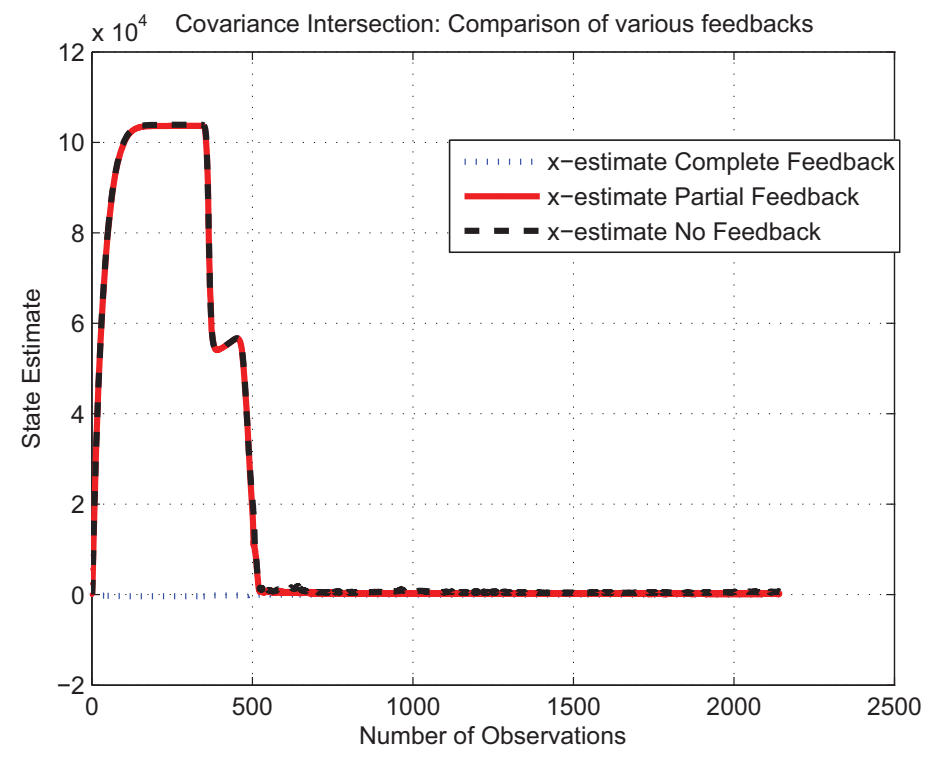

Figure 5.9: Covariance Intersection: Feedback Comparison 


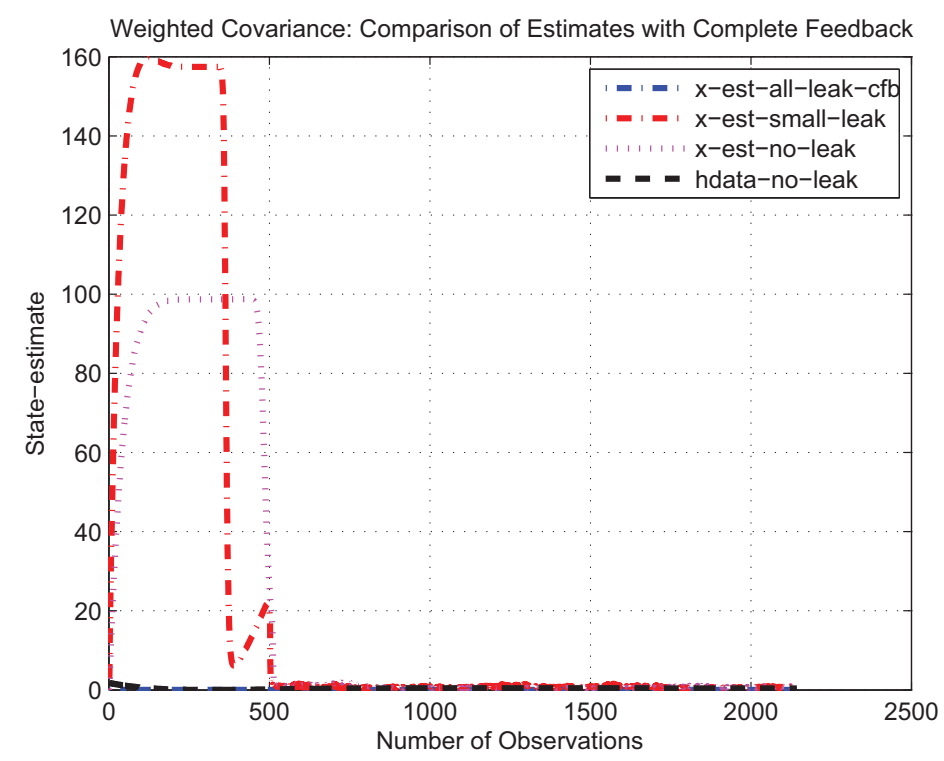

Figure 5.10: Weighted Covariance: Complete Feedback Comparison

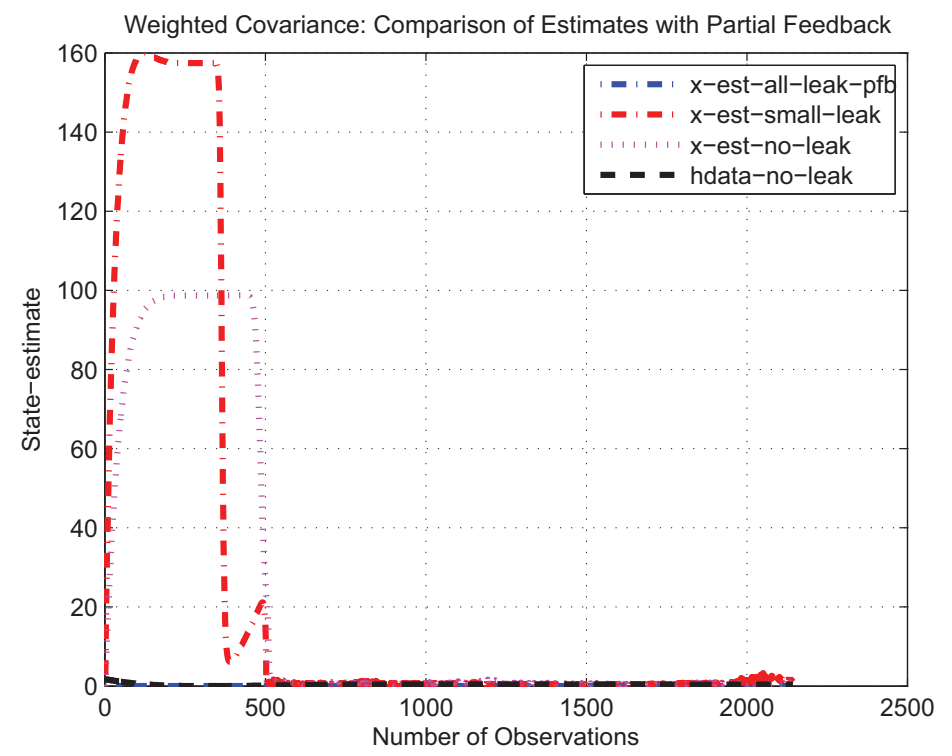

Figure 5.11: Weighted Covariance: Partial Feedback Comparison 


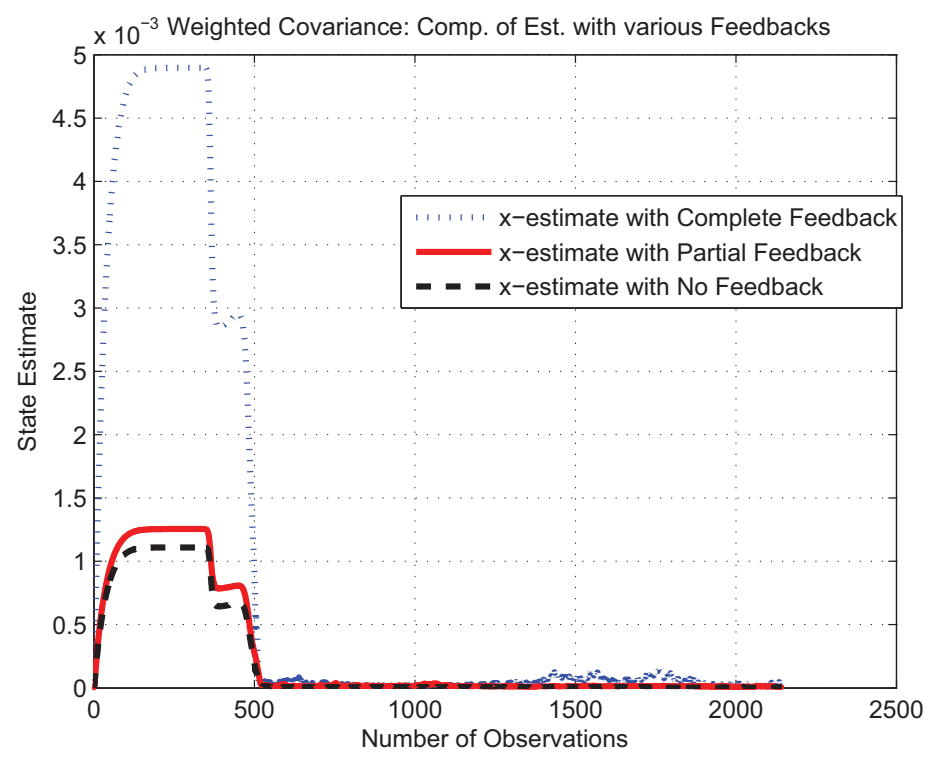

Figure 5.12: Weighted Covariance: Feedback Comparison

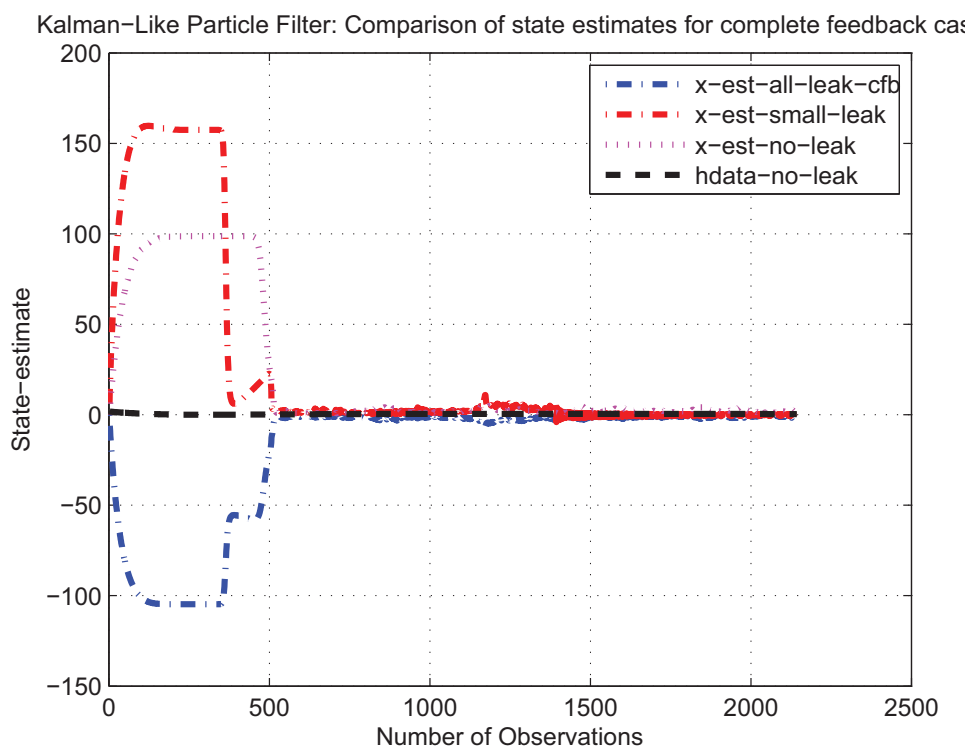

Figure 5.13: Kalman-like Particle Filter: Complete Feedback Comparison 


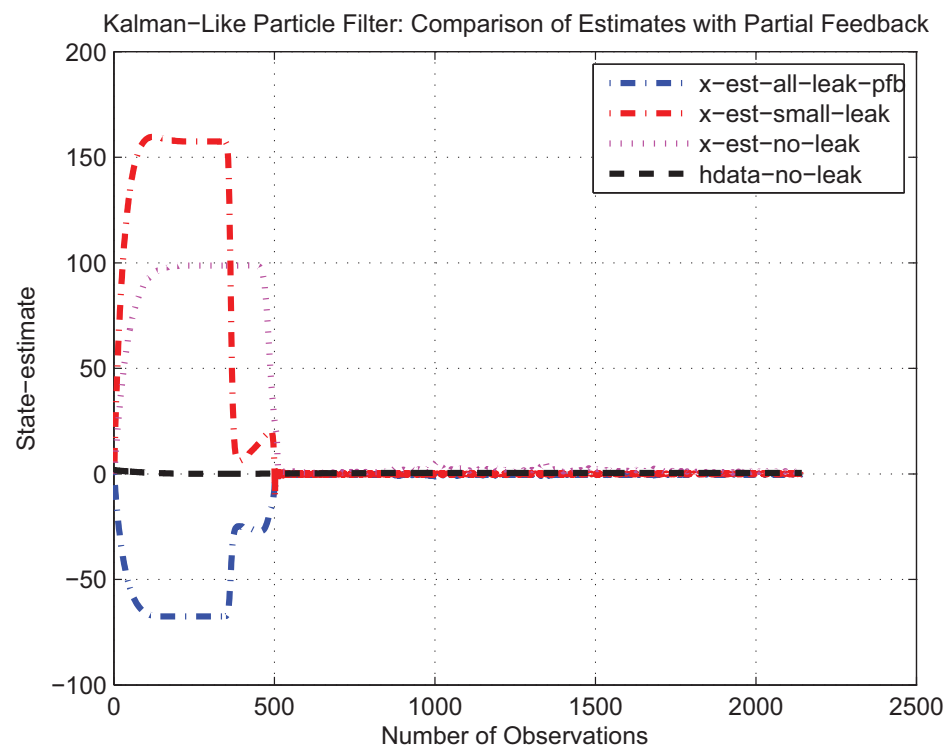

Figure 5.14: Kalman-like Particle Filter: Partial Feedback Comparison

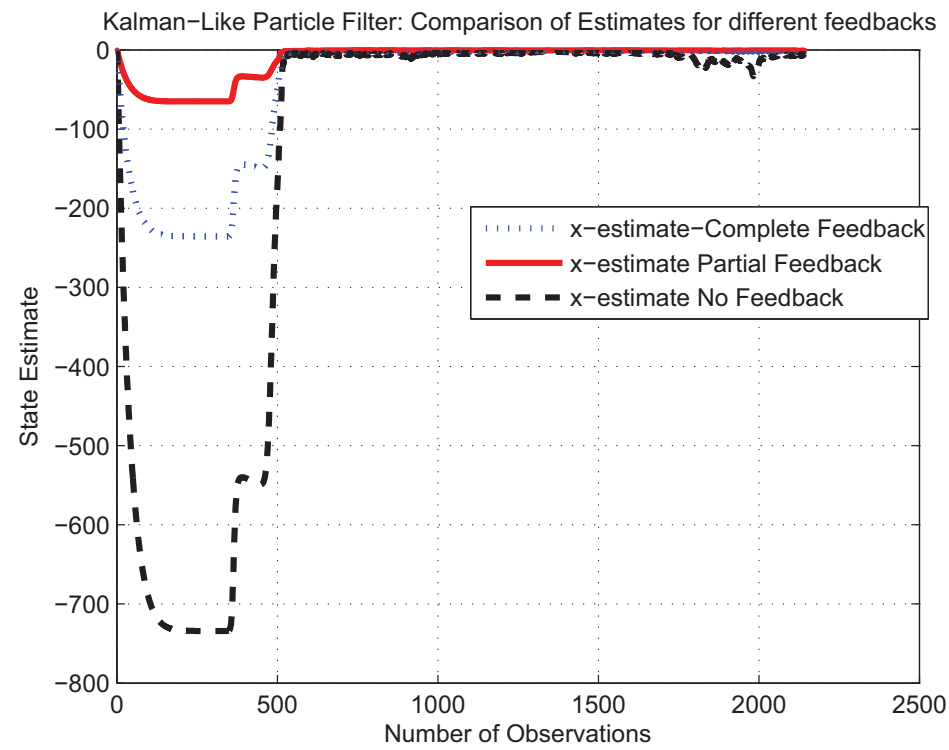

Figure 5.15: Kalman-like Particle Filter: Feedback Comparison 


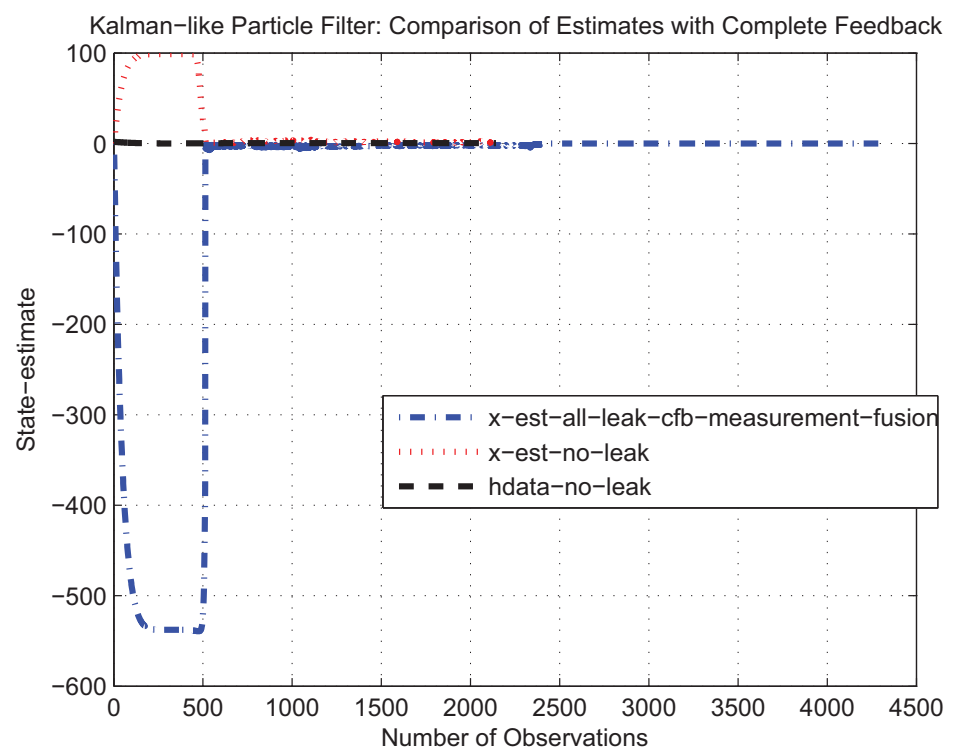

Figure 5.16: Kalman-like Particle Filter: Complete Feedback with measurement fusion method 


\section{Conclusions And Perspectives}

\subsection{Research CONCLUSions}

This dissertation proposes a different perspective for distributed Kalman filtering. It has the following conclusion.

Firstly, the distributed system architecture, on the whole, is very powerful since it allows the design of the individual units or components to be much simpler, while not compromising too much on the performance. A brief technical review and bibliography listing on the advances in DKF have been presented in the chapter 2. The current and previous approaches have been reported in this chapter. DKF comprising of OOSM approaches, Diffusion-Based approaches, Consensus Based Estimation, Self-Tuning designs and various applications of DKF have been classified. Some open problems and current research activities have been discussed and around 300 references have been categorized. We apologize in advance for any omission of publications, in spite of our best effort.

Secondly, approximate distributed estimation has been proposed in explicit forms using Bayesian-based FB Kalman filter for estimating states of a network control sys- 
tem for an arbitrary number of sensors with complete, incomplete, or no prior information. The approximate estimation presents all the prior cases with an effort to minimize time complexity and cases showing dependency of prior knowledge. Then, the algorithms were being made effective by data fusion of all the knowledge in a distributed filtering architecture. The proposed scheme has been evaluated on a rotational drive-based electro-hydraulic system using various fault scenarios, thus ensuring the effectiveness of the approach with different prior cases.

Thirdly, smoother extension to the SEEK filter with Kalman-like particle filter and EM implementation has been presented. The iterative process of EM helps the smoother to improve the covariance. The results show that the distributed filter of such kind has performed even better. Due to the EM implementation, the estimate almost mimics the original profile of the loads. The results have been then compared with the full-order version of such kind, thus ensuring its effectiveness.

Finally in the end, distributed estimation has been proposed using various versions of information matrix filter. Different feedback strategies were evaluated and the focal point is relation of performance and number of sensors. It is shown that for algorithms, the feedback strategies are performing differently i.e. information-based covariance intersection and Kalman-like particle filter is performing better with complete feedback case, whereas information-based weighted covariance is performing better with partial feedback case. The proposed scheme has been evaluated on a industrial boiler using fault scenarios, thus ensuring a thorough performance evaluation of the proposed filters with measurement fusion. 


\subsection{FUTURE RESEARCH WORK}

The following are the possible future research work which can be extended from this dissertation.

- DeVelopment of test Bed ShOWING APPROXimate ESTIMATION BASED ON PRIOR INFORMATION - A test bed to be designed based on real time prototype system for approximate estimation (using Bayesian-based Kalman filter), and the proposed approximate estimation filters with upper and lower bounds should be applied based on situations of complete, incomplete and no prior knowledge respectively. This estimation is to be extended to ensemble Kalman filtering presenting the case of large number of variables in interconnected system or multi-sensor data fusion.

- DeVElopMent of SMOOTHERS USING VARIOUS TYPE OF SigNALS SUCH AS ARMA SIGNALS - A robust smoother based on the a-priori knowledge of different signal types such as auto-regressive moving average (ARMA) and auto-regressive (AR) signals proposed to handle the smoothing process for every signal type.

- Development of a fault tolerant control scheme Considering DiSTRIBUTED ESTIMATION - Design of a fault tolerant control scheme which comprises of two steps, where the first step estimates and detects the error using a heavy non-linear a-priori knowledge-based filter, and the second step designs a reconfigurable controller which can control the plant with an error scenario being calculated from the filter in the first step. These type of systems are employed in mission critical situations, aircrafts etc. For example, during a flight of an aircraft, if one of the engine fails, how 
the second engine of the aircraft maneuvers itself to sustain a full load capacity of the plane and make a safe landing. 


\section{BIBLIOGRAPHY}

[1] Speyer, J. L., 'Computation and transmission requirements for a decentralized linear-quadratic-gaussian control problem', IEEE Trans. Autom. Control, vol. AC24(2), pp. 266-269, Apr. 1979.

[2] Olfati-Saber, R., 'Distributed Kalman filter with embedded consensus filter', In: Proc. 44th IEEE Conf. Decision and Control, European Control Conf., Dec. 2005, pp. 8179-8184.

[3] Nettleton, E., Durrant-Whyte, H., and Sukkarieh, S., 'A robust architecture for decentralised data fusion', presented at the Int. Conf. Advanced Robotics, 2003.

[4] Ribeiro, A., Giannakis, G., and Roumeliotis, S., 'SOI-KF: Distributed Kalman filtering with low-cost communications using the sign of innovations', IEEE Trans. Signal Process., vol. 54(12), pp. 4782-4795, Dec. 2006.

[5] Goshorn, R., Goshorn, J., Goshorn, D., and Aghajan, H., 'Architecture for clusterbased automated surveillance network for detecting and tracking multiple persons', In Proc. 1st IEEE/ACM Int. Conf. Distributed Smart Cameras, Sep. 2007, pp. 219226. 
[6] Luo, Y., Zhu, Y., Luo, D., Zhou, J., Song, E., and Wang, D., 'Globally optimal multisensor distributed random parameter matrices Kalman filtering fusion with applications', Sensors 2008, vol. 8, pp. 8086-8103.

[7] Luo, D. D., Zhu, Y. M., 'Applications of random parameter matrices Kalman filtering in Uncertain observation and multi-model systems', Proceedings of the International Federation of the Automatic Control, Seoul, Korea, July 6-11, 2008.

[8] Zhu, Y. M., You, Z. S., Zhao, J., Zhang, K. -S., and Li, r., X., 'The optimality for the distributed Kalman filtering fusion with feedback', Automatica, vol. 37, pp. 1489-1493, 2001.

[9] Jiang, T., Matei, I., Baras, J. S., 'A trust-based distributed Kalman filtering approach for mode estimation in power systems', Proceedings of the First Workshop on Secure Control Systems (SCS), pp. 1-6, Stockholm, Sweden, April 12, 2010.

[10] Saber, O., R., 'Distributed Kalman filtering for sensor networks', Proceedings of the 46th IEEE Conference on Decision and Control, pp. 5492-5498, 2007.

[11] Song, E. B., Zhu, Y. M., Zhou, J., You, Z. S., 'Optimality Kalman filtering fusion with cross-correlated sensor noises', Automatica 2007, vol. 43, pp. 1450-1456.

[12] Rao, B. S., Whyte, D-, H. F., 'Fully decentralized algorithm for multisensor Kalman filtering', IEE Proceedings on Control Theory and Applications, Sep. 1991; Part D, vol. 138, pp. 413-420.

[13] Chong, C. Y., Chang, K. C., Mori, S., 'Distributed tracking in distributed sensor networks', Proceedings of the American Control Conference, Seattle, 1986. 
[14] Hashmipour, H. R., Roy, S., Laub, A. J., 'Decentralized structures for parallel Kalman filtering', IEEE Trans. Autom. Control, 1988, vol. 33, pp. 88-93.

[15] Chang, K. C., Saha, R. K., Shalom, B., Y., 'On optimal track-to-track fusion', IEEE Trans. on Aerospace and Electronic Systems, vol. 33(4), Oct., 1997, pp. 1271-1276.

[16] Shalom, B., Y., Campo, L., 'The effect of the common process noise on the twosensor fused-track covariance', IEEE Trans. on Aerospace and Electronic Systems, vol. 22(6), 1986.

[17] Shalom, B., Y., Campo, L., 'The effect of the common process noise on the twosensor fused-track covariance', IEEE Trans. on Aerospace and Electronic Systems, vol. 22(6), pp. 803-805, 1986.

[18] Roecker, J. A., and McGillem, C. D., 'Comparison of two-Sensor tracking methods based on state vector fusion and measurement fusion', IEEE Trans. on AES, vol. 24(4), pp. 447-449, July 1988.

[19] Saha, R. K., and Chang, K. C., 'An efficient algorithm for multisensor track fusion', IEEE Trans on AES, vol. 34(1), January 1998, pp. 200-210.

[20] Saha, R. K., 'Track to track fusion with dissimilar sensors', IEEE Transactions on Aerospace and Electronic Systems, 1996, vol. 32(3), pp. 1021-1029.

[21] Shalom, B., Y., 'On the track-to-track correlation problem', IEEE Trans . on Automatic Control, 1981, vol. 26(2), pp. 571-572. 
[22] Kim, K. H., 'Development of track-to-track fusion algorithm', Proc. of American Control Conf., Troy : Rensselaer Polytechnic Institute, Maryland, 1994, pp. 10371041.

[23] Chen, H., Kirubarajan, T., Shalom, B., Y., 'Performance limits of track- totrack fusion vs. centralized estimation : theory and application', IEEE Trans . on Aerospace and Electronic Systems, 2003, vol. 39(2), pp. 386-398.

[24] Chen, H., Kirubarajan, T., Shalom, B., Y., 'Performance limits of track-to-track fusion vs. centralized estimation: theory and application', In: Proc. 4th Internat. Conf. on Information Fusion, Montreal, Quebec, 2001.

[25] Usman, A. K., Jose, M. F. M., 'Distributing the Kalman filter for large-scale systems', IEEE Transactions on Signal Processing, vol. 56(10), October 2008, pp. 4919-4935.

[26] Saligrama, V., and Castanon, D., 'Reliable distributed estimation with intermittent communications', In Proc. 45th IEEE Conf. Decision Control, San Diego, CA, Dec. 2006, pp. 6763-6768.

[27] Saber, O., R., 'Distributed Kalman filters with embedded consensus filters ', In Proc. 44th IEEE Conf. Decision Control, Seville, Spain, Dec. 2005, pp. 8179-8184.

[28] Saber, O., R., and Shamma, J., 'Consensus filters for sensor networks and distributed sensor fusion', In Proc. 44th IEEE Conf. Decision Control, Seville, Spain, Dec. 2005, pp. 6698-6703. 
[29] Kahn, U. A., and Moura, J. M. F., 'Distributed Kalman filters in sensor networks: bipartite fusion graphs', In Proc. 15th IEEE Workshop Statistical Signal Processing, Madison, WI, Aug. 2629, 2007, pp. 700-704.

[30] Xiao, L., and Boyd, S., 'Fast linear iterations for distributed averaging', Syst. Controls Lett., vol. 53(1), pp. 65-78, Apr. 2004.

[31] Carli, R., Chiuso, A., Schenato, L., and Zampieri, S., 'Distributed Kalman filtering based on consensus strategies', Information Engineering Dept., Univ. of Padova, Padova, Italy, Tech. Rep., 2007.

[32] Xiao, L., and Boyd, S., 'Designing fast distributed iterations via semi-definite programming', presented at the Workshop on Large Scale Nonlinear and Semidefinite Programming, Waterloo, Canada, May 2004.

[33] Kar, S., and Moura, J. M. F., 'Sensor networks with random links: Topology design for distributed consensus', IEEE Trans. Signal Process., vol. 56(7), pp. 33153326, Jul. 2008.

[34] Li, S., S., 'Optimal multi-sensor Kalman smoothing fusion for discrete multichannel ARMA signals', Journal of Control Theory and Applications, 2005, vol. 2, pp. $168-172$.

[35] Sun, S., 'Multi-sensor optimal information fusion Kalman filter for discrete multichannel ARMA signals', Proc. of 2003 IEEE Int. Symposium on Intelligent Control, Chicago : Institute of Electrical and Electronic Engineers Inc., Houston, October 2003 , pp. $377-382$. 
[36] Sun, S., Deng, Z., 'Multi-sensor optimal information fusion Kalman filter', Automatica, 2004, vol. 40(6), pp. 1017-1023.

[37] Li, S., S., 'Distributed optimal component fusion deconvolution filtering', Signal Processing, vol. 87, 2007, pp. 202-209.

[38] Hashmipour, H. R., Roy, S., Laub, A. J., 'Decentralized structures for parallel Kalman filtering', IEEE Trans. Automat. Control, vol. 33(1), 1988, pp. 88-93.

[39] Shalom, B., Y., 'On the track-to-track correlation problem', IEEE Trans. Automat. Control, vol. 26(2), 1981, pp. 571-572.

[40] Carlson, N. A., 'Federated square root filter for decentralized parallel processes', IEEE Trans. Aerospace Electron. Systems, pp. 26(3), (1990), pp. 517-525.

[41] Sun, S. L., 'Multi-sensor optimal information fusion Kalman filter with application', Aerospace Sci. Technol., vol. 8(1), (2004), pp. 57-62.

[42] Sun, S. L., Deng, Z. L., 'Multi-sensor optimal information fusion Kalman filter', Automatica, vol. 40(6), (2004), pp. 1017-1023.

[43] Sun, S. L., 'Multi-sensor information fusion white noise filter weighted by scalars based on Kalman predictor', Automatica, vol. 40(8), (2004), pp. 1447-1453.

[44] Sun, S. L., 'Multisensor optimal information fusion input white noise deconvolution estimators', IEEE Trans. Systems Man, Cybernet., vol. 34(4), (2004), pp. 1886-1893. 
[45] Li, S., S., Li, Z., D., 'Multi-sensor optimal information fusion Kalman filter', Automatica, vol. 40, 2004, pp. 1017-1023.

[46] Deng, Z. L., and Qi, R. B., 'Multi-sensor information fusion suboptimal steadystate Kalman filter', Chinese Science Abstracts, vol. 6(2), pp. 183-184, 2000.

[47] Qiang, G., and Harris, C. J., 'Comparison of two measurement fusion methods for Kalman filter-based multisensor data fusion', IEEE Transactions on Aerospace and Electronic Systems, vol. 37(1), 273280, 2001.

[48] Willner, D., Chang, C. B., Dunn, K. P., 'Kalman filter algorithm for a multisensor system’, In: Proc IEEE Conf. Decision and Control, 1976.

[49] Ling, S., Karl, H. J., and Richard, M. M., 'Kalman filtering with uncertain process and measurement noise covariances with application to state estimation in sensor networks', 16th IEEE International Conference on Control Applications, Part of IEEE Multi-conference on Systems and Control, Singapore, 1-3 October 2007, pp. $1031-1036$.

[50] Yang, W., Wendong, X. and Lihua, X., 'Diffusion-based EM algorithm for distributed estimation of gaussian mixtures in wireless sensor networks, Sensors 2011, vol. 11, pp. 6297-6316.

[51] Cattivelli, F., Sayed, A. H., 'Diffusion strategies for distributed Kalman filtering and smoothing', IEEE Trans. Autom. Control, 2010, vol. 55, pp. 2069-2084.

[52] Li, R., X., 'Optimal linear estimation fusion part VII: dynamic systems', Int. Conf. Information Fusion, Cairns, Australia,, July 2003, pp. 455-462. 
[53] Zhang, K. -S., Li, R., X., Zhang, P., and Li, H. -F., 'Optimal linear estimation fusionpart VI: sensor data compression', In: Proc. 2003 Int. Conf. Information Fusion, Cairns, Australia, July 2003.

[54] Li, R., X., Zhang, K. -S., Zhao, J., and Zhu, Y. -M., 'Optimal linear estimation fusionpart V: relationships', In: Proc. 2002 International Conf. on Information Fusion, pp. 497-504, Annapolis, MD, USA, July 2002.

[55] Li, R., X., and Zhang, K. -S., 'Optimal linear estimation fusionPart IV: optimality and efficiency of distributed fusion', In: Proc. 2001 Int. Conf. Information Fusion, Montreal, QC, Canada, Aug. 2001, pp. WeB1.19-WeB1.26.

[56] Li, R., X., and Zhang, P., 'Optimal linear estimation fusionPart III: crosscorrelation of local estimation errors', In: Proc. 2001 Int. Conf. Information Fusion, Montreal, QC, Canada, Aug. 2001, pp. WeB1.11-WeB1.18.

[57] Li, R., X., and Wang, J., 'Unified optimal linear estimation fusionPart II: discussions and examples', In: Proc. 2000 Int. Conf. Information Fusion, Paris, France, July 2000, pp. MoC2.18-MoC2.25.

[58] Li, R., X., Zhu, Y. M., and Han, C. Z., 'Unified optimal linear estimation fusionpart I: unified models and fusion rules', In: Proc. 2000 International Conf. on Information Fusion, pp. MoC2.10-MoC2.17, Paris, France, July 2000.

[59] Li, R., X., Y. M. Zhu, J. Wang, and C. Z. Han. 'Optimal linear estimation fusionpart I: unified fusion rules', IEEE. Trans. Information Theory, vol. 49(9), 2003, pp. 2192-2208. 
[60] Han, C. Z., Wang, J., and Li, R., X., 'Fused state estimation of linear continuoustime system with multi-sensor asynchronous measurements', In Proc. 2001 International Conf. on Information Fusion, pp. FrB1.3-FrB1.9, Montreal, QC, Canada, Aug. 2001.

[61] Zhang, K. S., Li, R., X., and Zhu, Y. M., 'Optimal update with out-of-sequence observations for distributed filtering', In: Proc. 2002 International Conf. on Information Fusion, pp. 1519-1526, Annapolis, MD, USA, July 2002.

[62] Keshu, Z., Li, R., X., and Yunmin, Z., 'Optimal update with out-of-sequence measurements for distributed filtering', ISIF, 2002, pp. 1519-1526.

[63] Shalom, B., Y., 'Update with out-of-sequence measurements in tracking: exact solution', In: Proc. 2000 SPIE Conf. on Signal and Data Processing of Small Targets, vol. 4048, Apr. 2000.

[64] Thomopoulos, S., and Zhang, L., 'Distributed filtering with random sampling and delay', In: Proc. 27th IEEE Conf. Decision and Control, (Austin, TX), Dec. 1988.

[65] Aleksandar, D., and Kun, Q., 'Decentralized random-field estimation for sensor networks using quantized spatially correlated data and fusion-center feedback', IEEE transactions on Signal Processing, vol. 56(12), December 2008, pp. 60696085.

[66] Dogandzic, A., and Qiu, K., 'Estimating a random field in sensor networks using quantized spatially correlated data and fusion-center feedback', In: Proc. 42nd 
Asilomar Conf. Signals, Syst. Comput., Pacific Grove, CA, Oct. 2008, pp. 19431947.

[67] Hong Z. Q., Hong Y. Z., Hong, J., 'Fusion algorithm of correlated local estimates', Aerospace Science and Technology, vol. 8, 2004, pp. 619-626.

[68] Sun, S- L., 'Multi-sensor optimal information fusion Kalman filters with applications', Aerospace Science and Technology, vol. 8, (2000), pp. 57-62.

[69] Oh, S., and Sastry, S., 'Distributed networked control system with lossy links: state estimation and stabilizing communication control', In: Proc. of the 45th IEEE Conference on Decision and Control, San Diego, CA, Dec. 2006.

[70] Oh, S., and Shankar S., 'Approximate estimation of distributed networked control systems', Proceedings of the 2007 American Control Conference, Marriott Marquis Hotel at Times Square, New York City, USA, July 11-13, 2007, pp. 997-1002.

[71] Chang, K. C., 'Evaluating hierarchical track fusion with information matrix filter', Proceedings of the Third International Conference on Information Fusion, 10-13 July 2000, vol. 1, pp. MOC2/3- MOC2/9.

[72] Shalom, B., Y., Campo, L., 'The effect of the common process noise on the twosensor fused-track covariance', IEEE Trans. on Aerospace and Electronic Systems, vol. 22(6), November, 1986, pp. 803-805.

[73] Saha, R. K., and Chang, K. C., 'An efficient algorithm for multi-sensor track Fusion', IEEE Trans on AES, vol. 34(1), January 1998, pp. 200-210. 
[74] Saha, R. K., and Chang, K. C., 'Centralized fusion of remote tracks', Optical Engineering, Special Section on Sensor Fusion, vol. 37(2), February 1998, pp. 468-476.

[75] Belkin, B., Anderson, S. L., and Sommar, K. M., 'The pseudo-measurement approach to track-to-track data fusion', In: Proc. 1993 Joint Service Data Fusion Symposium, pp 519-538, 1993.

[76] Drummond, O. E., 'Feedback in track fusion without process noise', In: Proc. SPIE Conference on Signal and Data Processing of Small Targets, vol. 2561, pp 369-383, 1995.

[77] Chang, K. C., Tian, Z., and Saha, R. K., 'Performance evaluation of track fusion with information filter', Proc. First International Conference on MultisourceMultisensor Information Fusion, pp. 648-654, Las Vegas, NV, July 1998.

[78] Mori, S., Chong, C. -Y., Chang, K. C., and Barker, W., 'Track association and track fusion with non-deterministic target dynamics', Proc. Second International Conference on Information Fusion, pp. 231-238, July 1999.

[79] Chong, C. -Y., Mori, S., Chang, K. C., and Barker, W., 'Architectures and algorithms for track association and fusion', Proc. Second International Conference on Information Fusion, pp. 239-246, July 1999.

[80] Saber, O., R., 'Distributed Kalman filtering and sensor fusion in sensor networks', Springer- Lecture Notes in Control and Information Sciences, 2006, vol. 331/2006, pp. 157-167, DOI: 10.1007/11533382-10. 
[81] Zhang, K., Li, X. R., and Zhu, Y., 'Optimal update with out-of-sequence measurements', IEEE Transactions on Signal Processing, June 2005, vol. 53(6), pp. 1992-2004.

[82] Shalom, B., Y., 'Update with out-Of-sequence measurements in tracking: exact solution', IEEE Trans. Aerosp. Electron. Syst., vol. 38(3), pp. 769-777, Jul. 2002.

[83] Shalom, B., Y., Mallick, M., Chen, H., and Washburn, R., 'One-step solution for the general out-of-sequence measurements problem in tracking', In: Proceedings of the 2002 IEEE Aerospace Conference, Big Sky, MT, pp. 1551-1559, Mar, 2002.

[84] Challa, S., Evans, R., and Legg, J., 'A fixed Lag smoothing framework for oosi problems', Commun. Inf. Syst., vol. 2(4), pp. 327-350, Dec. 2002.

[85] Challa, S., Evans, R., and Wang, X., 'A bayesian solution to out-Of-sequence measurements problem and its approximations', Inf. Fusion, vol. 4(3), September 2003, pp. 185-199.

[86] Challa, S., Wang, X., and Legg, J., 'track-to-track fusion using out-of-sequence tracks', In: Proc. Int. Conf. Inf. Fusion, Annapolis, MD, July 2002, pp. 919-926.

[87] Mallick, M., Krant, S. J., and Shalom, B., Y., 'Multi-sensor multi-target tracking using out-of-sequence measurements', In: Proc. Int. Conf. Inf. Fusion, Annapolis, MD, Jul. 2002, pp. 135-142.

[88] Orton, M., and Marrs, A., 'A Bayesian approach to multi-target tracking data fusion with out-of-sequence measurements', Proc. IEEE, Algorithms and Applications (Ref. No. 2001/174), IEE, 16-17 Oct. 2001, pp. 15/1-15/5. 
[89] Thomopoulos, S., and Zhang, L., 'Distributed filtering with random sampling and delay', In: Proc. 27th IEEE Conf. Decision Control, Austin, TX, Dec. 1988, vol. 3, pp. 2348-2353.

[90] Zhang, K. S., Li, R., X., and Zhu, Y. M., 'Optimal update with out-of-sequence measurements for distributed filtering', In: Proc. Int. Conf. Inf. Fusion, Annapolis, MD, Jul. 2002, pp. 1519-1526.

[91] Yunmin, Z., Xianrong, L., Li, R., X., and Juan, Z., 'Linear minimum variance estimation fusion', Science in China Ser. F Information Sciences, 2004, vol.47(6), pp. $728-740$.

[92] Yoon, J. H., and Shin, V., 'Comparison analysis of distributed receding horizon filters for linear discrete-time systems with uncertainties', International Journal of Systems Control, vol.1(2), 2010, pp. 48-56.

[93] Sun, S. L., 'Multi-sensor optimal information fusion Kalman filter with applications', Aerospace Science and Technology, 2004, vol. 8(1), pp. 57-62.

[94] Sun, S. L., and Deng, Z. L., 'Multisensor information fusion Kalman filter weighted by scalars for systems with colored measurement noises', Journ. Dynam. Syst, Measurem., Contr., vol. 127(12), pp. 663-667, 2005.

[95] Xiangdong, Q., and Kuochu, C., 'Information matrix fusion with feedback versus number of sensors', CiteSeerX, 10.1.1.63.6672. 
[96] Chang, K. C., 'Evaluating hierarchical fusion with information matrix filter', In: Proceedings of the 3rd International Information Fusion Conference, Paris, France: ISIF. 2000.

[97] Gu, D., 'Distributed EM algorithm for gaussian mixtures in sensor networks', IEEE Transactions on Neural Networks, vol. 19(7), July 2008, pp. 1154-1166.

[98] Sheng, Y., Hu, X., and Ramanathan, P., 'Distributed particle filter with GMM approximation for multiple targets localization and tracking in wireless sensor networks', In: Proc. 4th Int. Symp. Inf. Process. Sensor Netw., Los Angeles, CA, Apr. 2005, pp. 181-188.

[99] Saber, O., R., 'Distributed Kalman filter with embedded consensus filters', In: Proc. 44th IEEE Conf. Decision Control, Dec. 1215, 2005, pp. 8179-8184.

[100] Sheng, Y., Hu, X., and Ramanathan, P., 'Distributed particle filter with GMM approximation for multiple targets localization and tracking in wireless sensor networks', Fourth International Symposium on Information Processing in Sensor Networks, April 2005.

[101] Spanos, D. P., Olfati-Saber, R., and Murray, R. M., 'Approximate distributed Kalman filtering in sensor networks with quantifiable performance', Fourth International Symposium on Information Processing in Sensor Networks (IPSN 05), pp. 133-139, April 2005.

[102] Matei, I., and Baras, J. S., 'Consensus-based linear distributed filtering', The Institute for Systems Research, ISR Technical Report 2010-5. 
[103] Carli, R., Chiuso, A., Schenato, L., and Zampieri, S., 'Distributed Kalman filtering based on consensus strategies', IEEE Journal on selected area in communication, vol. 26(4), pp. 622-633, May 2008.

[104] Saber, O., R., 'Distributed Kalman filter with embedded consensus filters', In: Proc. of 44th IEEE Conf. on Dec., and the Eur. Contr. Conf., Seville, Spain, 2005, pp. $8179-8184$.

[105] Barooah1, P., Russell, W. J., and Hespanha, J. P., 'Approximate distributed Kalman filtering for cooperative multi-agent localization', Proceedings of the 6th IEEE international conference on Distributed Computing in Sensor Systems, DCOSS'10, Santa Barbara, CA, 2010, pp. 102-115.

[106] Alriksson, P., Rantzer, A., 'Distributed Kalman filtering using weighted averaging', In: 17th International Symposium on Mathematical Theory of Networks and Systems (MTNS), (2006).

[107] Saber, O., R. 'Distributed Kalman filtering for sensor networks', In: 46th IEEE Conference on Decision and Control, (December 2007), pp. 5492-5498

[108] Carli, R., Chiuso, A., Schenato, L., Zampieri, S., 'Distributed Kalman filtering using consensus strategies', In: 46th IEEE Conference on Decision and Control, (December 2007), pp. 5486-5491

[109] Alriksson, P., Rantzer, A., 'Experimental evaluation of a distributed Kalman filter algorithm', In: 46th IEEE Conference on Decision and Control, (December 2007), pp. 5499-5504. 
[110] Stankovic, S. S., Stankovic, M. S., and Stipanovic, D. M., 'Consensus based overlapping decentralized estimator', IEEE Transactions on Automatic Control, Feb. 2009, vol. 54(2), pp. 410-415.

[111] Saber, O., R., and Shamma, J. S., 'Consensus filters for sensor networks and distributed sensor fusion,' 44th IEEE Conference on Decision and Control, 2005 and 2005 European Control Conference, CDC-ECC '05, 12-15 Dec. 2005, pp. $6698-6703$.

[112] Saber, O., R., and Murray, R. M., 'Consensus problems in networks of agents with switching topology and time-delays', IEEE Trans. on Automatic Control, vol. 49(9), pp. 1520-1533, Sep. 2004.

[113] Saber, O., R., and Murray, R. M., 'Consensus protocols for networks of dynamic agents', Proc. of the American Control Conference, vol. 2, pp. 951-956, June 2003.

[114] Ogren, P., Fiorelli, E., and Leonard, N. E., 'Cooperative control of mobile sensor networks: adaptive gradient climbing in a distributed GMM approximation for multiple targets localization and tracking in wireless sensor networks', Fourth International Symposium on Information Processing in Sensor Networks, April 2005.

[115] Li, R., X., 'Optimal linear estimation fusion for multi-sensor dynamic Systems', Proc. Workshop on Multiple Hypothesis Tracking - A Tribute to Sam Blackman, San Diego, CA, USA, May 2003. 
[116] Zhang, K. -S., Li, R., X., and Zhu, Y. -M., 'Optimal update with out-of-sequence observations for distributed filtering', IEEE Transactions on Signal Processing, vol. 53(6), pp. 1992-2004, June 2005.

[117] Zhu, Y., Gana, Yu, Li, R., X., 'Multisensor statistical interval estimation fusion', Proc. 2002 SPIE Conf. Sensor Fusion: Architectures, Algorithms, and Applications VI, vol. 47, pp. 31-36, Orlando, FL, April 2002.

[118] Mohammadi, A., and Asif, A., 'Consensus-based disributed unscented particle filter', 2011 IEEE Statistical Signal Processing Workshop (SSP), pp. 28-30, June 2011, pp. 237-240.

[119] Coates, M. J., and Oreshkin, B. N., 'Asynchronous distributed particle filter via decentralized evaluation of gaussian products', In: Proc. ISIF Int. Conf. Inf. Fusion, 2010.

[120] Gu, D., Junxi, S., Zhen, H., and Hongzuo, L., 'Consensus based distributed particle filter in sensor networks', In: IEEE Int. Conference on Information and Automation, 2008, pp. 302-307.

[121] Saber, O., R., Fax, A., and Murry, R. M., 'Consensus and coopration in networked multi-agent systems', In: Proceedings of the IEEE, 2007.

[122] Dimakis, A. G., Kar, S., Moura, J. M. F., Rabbat, M. G., and Scaglione, A., 'Gossip algorithms for distributed signal processing', Proc. of the IEEE, vol. 98, pp. 1847-1864, 2010. 
[123] Alriksson, P., and Rantzer, A., 'Distributed Kalman filtering Using Weighted Averaging', 17th International Symposium on Mathematical Theory of Networks and Systems, 2006, Kyoto, Japan.

[124] Chang, K., Zhi, T., and Saha, R., 'Performance evaluation of track fusion with information matrix filter', IEEE Transactions on Aerospace and Electronic Systems, vol. 38(2), pp. 455-466, 2002.

[125] Duan, Z., Rong-Li, X., 'Optimal distributed estimation fusion with transformed Data', 2008 11th International Conference on Information Fusion, June 30-July 3, 2008, pp. 1291-1297.

[126] Chen, H. M., Kirubarajan, T., and Bar-Shalom, Y., 'Performance limits of trackto-track fusion versus centralized estimation: theory and application', IEEE Transactions on Aerospace and Electronic Systems, vol. 39(2), pp. 386-400, April 2003.

[127] Chang, K. C., Tian, Z., and Mori, S., 'Performance evaluation for MAP state estimate fusion', IEEE Transactions on Aerospace and Electronic Systems, vol. 40(2), pp. 706-714, April 2004.

[128] Duan, Z., Li, R., X., 'The optimality of a class of distributed estimation fusion algorithm', The 11th International Conference on Information Fusion, 2008, pp. $1285-1290$.

[129] Xu, J., Song, E., Luo, Y., and Zhu, Y., 'Optimal distributed Kalman filtering fusion algorithm Without Invertibility of estimation error and sensor noise covariances', IEEE Signal Processing Letters, vol. 19(1), January 2012, pp. 55-58. 
[130] Shen, X., Zhu, Y., Song, E., and Luo, Y., 'Minimizing euclidian state estimation error for linear Uncertain dynamic systems based on multi-sensor and multialgorithm fusion', IEEE Transactions on Information Theory, vol. 57(10), October 2011, pp. 7131-7146.

[131] Noack, B., Lyons, D., Nagel, M., and Hanebeck, U. D., 'Non-linear information filtering for distributed multi-sensor data fusion', 2011 American Control Conference, on O'Farrell Street, San Francisco, CA, USA, June 29 - July 01, 2011, pp. 4846-4852.

[132] Gasparri, A., Pascucci, F., and Ulivi, G., 'A distributed extended information filter for self-localization in sensor networks', In: Proceedings of IEEE 19th International Symposium on Personal, Indoor and Mobile Radio Communications (PIMRC 2008), Sept. 2008, pp. 1-5.

[133] Ong, L., -L., Upcroft, B., Ridley, M., Bailey, T., Sukkarieh, S., and Whyte, D., H., 'Consistent Methods for Decentralised Data Fusion using particle filters', Proceedings of the 2006 IEEE International Conference on Multisensor Fusion and Integration for Intelligent Systems (MFI 2006), pp. 85-91, 2006.

[134] Mohammadi, A., and Asif, A., 'Consensus-based distributed unscented particle filter', 2011 IEEE Statistical Signal Processing Workshop (SSP), pp. 237-240.

[135] Coates, M. J., and Oreshkin, B. N., 'Asynchronous distributed particle filter via decentralized evaluation of gaussian products', In: Proc. ISIF Int. Conf. Inf. Fusion, 2010. 
[136] Gu, d., Junxi, S., Zhen, H., and Hongzuo, L., 'Consensus based distributed particle filter in sensor networks', In: IEEE Int. Conference on Information and Automation, 2008, pp. 302-307.

[137] Oreshkin, B. N., Liu, X., and Coates, M. J., 'Efficient delay-tolerant particle filtering', IEEE Transactions on Signal Processing, vol. 59(7), July 2011, pp. 3369_ 3381.

[138] Shalom, B., Y., 'Update with out-of-sequence measurements in tracking: exact solution', IEEE Trans. Aerosp. Electron. Syst., vol. 38(3), pp. 769-778, Mar. 2002.

[139] Shalom, B., Y., Chen, H., and Mallick, M., 'One-step solution for the multistep out-of-sequence-measurement problem in tracking', IEEE Trans. Aerosp. Electron. Syst., vol. 40(1), pp. 27-37, Jan. 2004.

[140] Zhang, K., Rong-Li, X., and Zhu, Y., 'Optimal update with out-of-sequence measurements’, IEEE Trans. Signal Process., vol. 53(6), pp. 1992-2004, Jun. 2005.

[141] Maskell, S. R., Everitt, R. G., Wright, R., and Briers, M., 'Multi-target out-ofsequence data association: tracking using graphical models', Inf. Fusion, vol. 7(4), pp. 434-447, Dec. 2006.

[142] Shen, X., Zhu, Y., Song, E., and Luo, Y., 'Optimal centralized update with multiple local out-of-sequence measurements', IEEE Trans. Signal Process., vol. 57(4), pp. 1551-1562, Apr. 2009. 
[143] Zhang, S., Shalom, B., Y., and Watson, G., 'Tracking with multisensor out-ofsequence measurements with residual biases', In: Proc. ISIF Int. Conf. Inf. Fusion, Edinburgh, U.K., Jul. 2010.

[144] Orton, M., and Marrs, A., 'Storage efficient particle filters for the out-ofsequence measurement problem', In: Proc. IEE Colloq. Target Tracking: Algorithms and Appl., Enschede, The Netherlands, Oct. 2001.

[145] Orton, M., and Marrs, A., 'Particle filters for tracking with out-of-sequence measurements', IEEE Trans. Aerosp. Electron. Syst., vol. 41(2), pp. 693-702, Feb. 2005.

[146] Mallick, M., Kirubarajan, T., and Arulampalam, S., 'Out-of-sequence measurement processing for tracking ground target using particle filters', In: Proc. IEEE Aerosp. Conf., Mar. 2002, vol. 4, pp. 1809-1818.

[147] Zhang, W., Huang, X., and Wang, M., 'Out-of-sequence measurement algorithm based on Gaussian particle filter', Inf. Technol. J., vol. 9(5), pp. 942-948, May 2010.

[148] Orguner, U., and Gustafsson, F., 'Storage efficient particle filters for the out-ofsequence measurement problem', In: Proc. ISIF Int. Conf. Inf. Fusion, Cologne, Germany, Jul. 2008.

[149] Tasoulis, D. K., Adams, N. M., and Hand, D. J., 'Selective fusion of out-ofsequence measurements', Inf. Fusion, vol. 11(2), pp. 183-191, Apr. 2010.

[150] Liu, X., Oreshkin, B. N., and Coates, M. J., 'Efficient delay-tolerant particle filtering through selective processing of out-of-sequence measurements', In: Proc. ISIF Int. Conf. Inf. Fusion, Edinburgh, U.K., Jul. 2010. 
[151] Jiang, P., Zhou, J., and Zhu, Y., 'Globally optimal Kalman filtering with finitetime correlated noises', 49th IEEE Conference on Decision and Control, December 15-17, 2010, Hilton Atlanta Hotel, Atlanta, GA, USA, pp. 5007-5012.

[152] Govaers, F., and Koch, W., 'Distributed Kalman filter fusion at arbitrary instants of time', 2010 13th Conference on Information Fusion (FUSION), 26-29 July 2010, Sensor Data and Inf. Fusion, Univ. of Bonn, Bonn, Germany, pp. 1-8.

[153] Koch, W., 'Exact update formulae for distributed Kalman filtering and retrodiction at arbitrary communication rates', In: Proc. of the 12th ISIF International Conference on Information Fusion, FUSION 2009, Seattle, USA, July 6-9, 2009.

[154] Zheng, S., Jiang, T., Baras, J. S., 'Robust state estimation Under false data injection in distributed sensor networks', IEEE Globecom 2010 Proceedings, Miami, Florida, December 6-10, 2010, pp. 1-5.

[155] Saber, O., R., 'Distributed Kalman filter with embedded consensus filters', In: Proceedings of 44th IEEE Conference on Decision and Control, 2005 and 2005 European Control Conference (CDC-ECC 05), 2005.

[156] Wang, D., Niu, K., He, Z., Tian, B., 'A novel OFDM channel estimation method based On Kalman filtering and distributed compressed sensing', 2010 IEEE 21st International Symposium on Personal Indoor and Mobile Radio Communications, pp. 1080-1090. 
[157] Gui-Li, T., Xue, H., G., Zi-Li, D., 'Self-tuning distributed fusion Kalman filter With asymptotic global optimality', Proceedings of the 29th Chinese Control Conference, July 29-31, 2010, Beijing, China, pp. 1268-1272.

[158] Deng, Z. L., Gao, Y., Mao, L., Li, Y., and Hao, G., 'New approach to information fusion steady-state Kalman filtering', Automatica, 2005, vol. 41(10), pp. 16951707.

[159] Deng, Z. L., and Li, C. B., 'Self-tuning information fusion Kalman predictor weighted by diagonal matrices and its convergence anaysis', Acta Automatic Sinica, 2007, vol. 33(2), pp. 156-163.

[160] Ran, C. J., Tao, G. L., Liu, J. F., and Deng, Z. L., 'Self-tuning decoupled fusion Kalman predictor and its convergence analysis', IEEE Sensors Journal, 2009, vol. 9(12), pp. 2024-2032.

[161] Gao, Y., Jia, W. J., Sun, X. J., and Deng, Z. L., 'Self-tuning multisensor weighted measurement fusion Kalman filter', IEEE Tran. Aerospace and Electronic System, 2009, vol. 45(1), 179-191.

[162] Yingting, L., and Yunmin, Z., 'Distributed Kalman filtering fusion with packet loss or intermittent communications from local estimators to fusion Center', Proceedings of the 29th Chinese Control Conference, July 29-31, 2010, Beijing, China, pp. $4768-4775$.

[163] Shen, X., Zhu, Y., and You, Z., 'Optimal flight path update with adding or removing out-of-sequence measurements', International Conference on Intelligent 
Control and Information Processing, August 13-15, 2010 - Dalian, China, pp. 427432.

[164] Shalom, B., Y., Chen, H., 'Removal of out-of-sequence measurements from tracks', IEEE Transactions on Aerospace and Electronic Systems, vol. 45, pp. 612$619,2009$.

[165] Challa, S., Evans, R. J., and Wang, X., 'Track-to-track fusion using out-ofsequence tracks', Proceedings of the Fifth International Conference on Information Fusion, Annapolis, MD, pp. 919-926, 2002.

[166] Challa, S., Evans, R. J., Wang, X., and Legg, J., 'A fixed lag smoothing framework for OOSM problems', Communications in Information and Systems, vol. 2(4), 327-350, 2002.

[167] Rothrock, R., 'The need to remove out-of-sequence measurements', Presented at the Tracking and CID Alternatives Identification and Evaluation Team (TCAIET) Meeting, Arlington, VA, 2006.

[168] Shen, X. J., Zhu, Y. M., Song, E. B., and Luo, Y. T., 'Globally optimal distributed Kalman fusion with local out-of-sequence-measurement updates', IEEE Transactions on Automatic Control, vol. 54, pp. 1928-1934, 2009.

[169] Teng, Z., Jing, M., and Shuli, S., 'Distributed fusion filter for discrete-time stochastic systems with Uncertain observation and correlated noises', 2010 8th IEEE International Conference on Control and Automation, Xiamen, China, June 9-11, 2010, pp. 704-708. 
[170] Sun, S. L., 'Multi-sensor optimal information fusion Kalman filter with application', Aerospace Science and Technology, vol. 8(1), pp. 57-62, Jan. 2004.

[171] Sun, S. L., Deng, Z. L., 'Multi-sensor optimal information fusion Kalman filter', Automatica, vol. 40(6), pp. 1017-1023, Jun. 2004.

[172] Sun, S. L., 'Multi-sensor optimal information fusion input white noise deconvolution estimators', IEEE Transactions on Systems, Man, And Cybernetics, vol. 34(4), pp. 1886-1893, Aug 2004.

[173] Cattivelli, F., Sayed, A. H., 'Diffusion distributed Kalman filtering with adaptive Weights', 2009 Conference Record of the Forty-Third Asilomar Conference on Signals, Systems and Computers, pp. 908-912, 1-4 November 2009.

[174] Cattivelli, F. S., Lopes, C. G., and Sayed, A. H., 'Diffusion strategies for distributed Kalman filtering: formulation and performance analysis', In: Proc. Cognitive Information Processing, Santorini, Greece, June 2008.

[175] Cattivelli, F. S., and Sayed, A. H., 'Diffusion mechanisms for fixed-point distributed Kalman smoothing', In: Proc. EUSIPCO, Lausanne, Switzerland, August 2008, [CD ROM].

[176] Schizas, I., Roumeliotis, S. I., Giannakis, G. B., and Ribeiro, A., 'Anytime optimal distributed Kalman filtering and smoothing', In: Proc. IEEE Workshop on Statistical Signal Process., Madison, WI, August 2007, pp. 368-372. 
[177] Khan, U. A., and Moura, J. M. F., 'Distributing the Kalman filter for large scale systems', IEEE Trans. on Signal Processing, vol. 56(10), Part 1, pp. 4919-4935, October 2008.

[178] Cattivelli, F. S., and Sayed, A. H., 'Diffusion strategies for distributed Kalman filtering and smoothing', IEEE Transactions on Automatic Control, vol. 55(9), September 2010, pp. 2069-2084.

[179] Alriksson, P., and Rantzer, A., 'Distributed Kalman filtering using weighted averaging', In: Proc. 17th Int. Symp. Math. Theory Networks Syst., Kyoto, Japan, Jul. 2006, [CD ROM].

[180] Carli, R., Chiuse, A., Schenato, L., and Zampieri, S., 'Distributed Kalman filtering based on consensus strategies', IEEE J. Selected Areas Commun., vol. 26(4), pp. 622-633, May 2008.

[181] Sukhavasi, R. T., and Hassibi, B., 'The Kalman like particle filter : optimal estimation With quantized innovations/measurements', Joint 48th IEEE Conference on Decision and Control and 28th Chinese Control Conference, Shanghai, P.R. China, December 16-18, 2009, pp. 4446-4451.

[182] Ran, C., and Deng, Z., 'Self-tuning Weighted measurement fusion Kalman filter and its convergence snalysis', Joint 48th IEEE Conference on Decision and Control and 28th Chinese Control Conference, Shanghai, P.R. China, December 16-18, 2009, pp. 1830-1835. 
[183] Gao, Y., Ran, C. J., and Deng, Z. L., 'Weighted measurement fusion Kalman filter with correlated measurement noise and its global optimality', Proceedings of the International colloquiumen information Fusion 2007, Xian, China, August 22252007 , pp. 195-200.

[184] Deng, Z. L., Gao, Y., Li, C. B., and Hao, G., 'Self-tuning decoupled information fusion Wiener state component filters and their convergence', Automatica, vol. 44, pp. 685-695, 2008.

[185] Sun, X. J., Zhang, P., and Deng, Z. L., 'Self-tuning decoupled fusion Kalman filter based on Riccati equation', Frontiers of Electrical and Electronic Engineering in China, vol. 3(4), pp. 459-464, 2008.

[186] Cortes, J., 'Distributed kriged Kalman filter for spatial estimation', IEEE Transactions on Automatic Control, vol. 54(12), December 2009, pp. 2816-2827.

[187] Wen, C. L., Ge, Q. B., and Feng, X. L., 'Optimal recursive fusion estimator for asynchronous system', Proceedings of the 7th Asian Control Conference, Hong Kong, China, August 27-29, 2009, pp. 148-153.

[188] Zhou, W., Li, L., and Chen, G., and Yu, A., 'Optimality analysis of one-step OOSM filtering algorithms in target tracking', Science in China Series F: Information Sciences, vol. 50(2), pp.170-187, 2007.

[189] Aounallah, F., Amara, R., and Alouane, M. T. H., 'Particle filtering based on sign of innovation for tracking a jump karkovian motion in a binary WSN', 2009 Third International Conference on Sensor Technologies and Applications, pp. 252-255. 
[190] Aounallah, F., Amara, R., and Alouane, M. T. -H., 'Particle filtering based on sign of innovation for distributed estimation in binary wireless sensor networks', IEEE Workshop SPAWC2008, Recife, Brasil, July 2008.

[191] Bickson, D., Shental, O., Dolev, D., 'Distributed Kalman filter via gaussian belief propagation', Forty-Sixth Annual Allerton Conference, Allerton House, UIUC, Illinois, USA September 23-26, 2008, pp. 628-635.

[192] Dey, S., Leong, A. S., and Evans, J. S., 'On Kalman filtering With faded measurements', Forty-Sixth Annual Allerton Conference, Allerton House, UIUC, Illinois, USA, September 23-26, 2008, pp. 607-614.

[193] Berg, T. M., and Durrant-Whyte, H. F., 'Model distribution in decentralized multi-sensor data fusion', American Control Conference, pp. 2292-2293, 26-28 June 1991.

[194] Shen, X., Zhu, Y., Song, E., and Luo, Y., 'Optimal centralized update With multiple local out-of-sequence measurements', IEEE Transactions on Signal Processing, vol. 57(4), April 2009, pp. 1551-1562.

[195] Challa, S., Evans, R. J., and Wang, X., 'Target tracking in clutter using timedelayed out-of-sequence measurements', In: Proc. Defence Applicat. Signal Process. (DASP), Sep. 2001.

[196] Ahmed, K. H., Massoud, A. M., Finney, S. J., and Williams, B. W., 'Sensorless modified adaptive Kalman filter for current control of a three-phase inverter based 
distributed generation', IEEE International Symposium on Industrial Electronics, pp. 1592-1597, June 30-July 22008.

[197] Schwarzenberg, G. F., Mayer, U., Ruiter, N. V., and Hanebeck, U. D., ‘3D reflectivity reconstruction by Means of spatially distributed Kalman Filters', Proceedings of IEEE International Conference on Multisensor Fusion and Integration for Intelligent Systems, Seoul, Korea, August 20 - 22, 2008, pp. 384-391.

[198] Aounallah, F., Amara, R., and Alouane, M. T. H., 'Particle filtering based on sign of innovation for distributed estimation in binary wireless sensor networks', SPAWC 2008, pp. 629-633.

[199] Medeiros, H., Park, J. and Kak, A. C., 'Distributed object tracking Using a cluster-based Kalman filter in wireless camera networks', IEEE Journal of Selected Topics in Signal Processing, vol. 2(4), August 2008, pp. 448-463.

[200] Zhu, B., and Sastry, S., 'Data fusion assurance for the Kalman filter in Uncertain networks', The Fourth International Conference on Information Assurance and Security, pp. 115-119.

[201] Mosquera, C., Jayaweera, S. K., 'Entangled Kalman filters for cooperative estimation', 5th IEEE SAM Sensor Array and Multichannel Signal Processing Workshop, 21-23 July 2008, pp. 279-283.

[202] Saber, O., R., 'Distributed Kalman filter with consensus filters', In: Proceedings of the 44th Em-bedded IEEE Conference on Decision and Control, Sevilla, Spain, Dec 2005, pp. 8179-8184. 
[203] Kirti, S., and Scaglione, A., 'Scalable distributed Kalman filtering through consensus', In: Proceedings of the IEEE International Conference on Acoustics, Speech and Signal Processing, ICASSP, April 2008.

[204] Saber, O. R., 'Distributed Kalman filter for sensor networks', In: Proceedings of the 46th IEEE Conferenceon Decision and Control, New Orleans, Dec 2007, pp. $5492-5498$.

[205] Alriksson, P., and Rantzer, A., 'Experimental evaluation of a distributed Kalman filter algorithm', In: Proceedings of the 46th IEEE Conference on Decision and Control, 2007.

[206] Schenato, L., Carli, R., Chiuso, A., and Zampieri, S., 'Distributed Kalman filtering based on consensus strategies', IEEE Journal on Selected Areas in Communications, vol. 26(4), pp. 622-633, May 2008.

[207] Wang, D., Zhu, Y., Shen, X., 'Distributed multi-sensor estimation fusion with out-of-sequence measurements', The 2008 International Conference on Embedded Software and Systems (ICESS2008), pp. 390-395.

[208] Shalom, B. Y., 'Update with out-of-sequence measurements in tracking: exact solution', IEEE Transactions on Aerospace and Electronic Systems, vol. 38(3), pp. 769-778, July 2002.

[209] Saber, O. R., and Nils F. Sandell, 'Distributed tracking in sensor networks with limited sensing range', 2008 American Control Conference, Westin Seattle Hotel, Seattle, Washington, USA, June 11-13, 2008, pp. 3157-3162. 
[210] Saber, O. R., 'Distributed Kalman filtering algorithms for sensor networks', Proceedings of the 46th IEEE Conference on Decision and Control, Dec. 2007.

[211] Msechu, E. J., Ribeiro, A., Roumeliotis, S. I., and Giannakis, G. B., 'Distributed Kalman filtering based on quantized innovations', ICASSP 2008, pp. 3293-3296.

[212] Msechu, E. J., Roumeliotis, S. I., Ribeiro, A., and Giannakis, G. B., 'Distributed quantized Kalman filtering with scalable communication cost', IEEE Trans. on Sig. Proc., Aug. 2007 (submitted), available at http://spincom.ece.umn.edu/journal.html.

[213] Kirti, S., and Scaglione, A., 'Scalable distributed Kalman filtering through consensus', ICASSP 2008, pp. 2725-2728.

[214] Saber, O., R., 'Distributed Kalman filter with embedded consensus filters', In: Proc. 44th IEEE Conference on Decision and Control, Dec. 2005, pp. 8179-8184,

[215] Spanos, D. P., Saber, O., R., and Murray, R., 'Approximate distributed Kalman filtering in sensor networks with quantifiable performance', In: Proc. 4th Int. Symposium on Information Processing in Sensor Networks, 2005, pp. 133-139.

[216] Msechu, E. J., Roumeliotist, S. I., Ribeiro, A., and Giannakis, G. B., 'Distributed iteratively quantized Kalman filtering for Wireless sensor networks', pp. 646-650.

[217] Schizas, I. D., Giannakis, G. B., Roumeliotis, S. I., and Ribeiro, A., 'Consensus in ad hoc WSNs With noisy linkspart II: distributed estimation and smoothing of random signals', IEEE Transactions on Signal Processing, vol. 56(4), April 2008, pp. 1650-1666. 
[218] Fong, L. W., 'Multi-sensor track-to-track fusion Using simplified maximum likelihood estimator for maneuvering target tracking', 2007 IEEE, pp. 36-41.

[219] Chang, K. C., Saha, R. K., and Shalom, B.,Y., 'On optimal track-to-track fusion', IEEE Transactions on Aerospace and Electronic Systems, vol. 33(4), pp. 12711276, May 1997.

[220] Fong, L. W., 'Integrated track-to-track fusion with modified probabilistic neural network', 2006 CIE International Conference on Radar, Shanghai China, pp. 1869-1873, Oct. 2006.

[221] Schizas, I. D., Giannakis, G. B., Roumeliotis, S. I., and Ribeiro, A., 'Anytime optimal distributed Kalman filtering and smoothing', SSP 2007, pp. 368-372.

[222] Spanos, D. P., Saber, O., R., and Murray, R. J., 'Approximate distributed Kalman filtering in sensor networks with quantifiable performance', In: Proc. of the 4th Intl. Symp. on Info. Proc. in Sensor Networks, Los Angeles, CA, 2005.

[223] Zhu, B., Sinopoli, B., Poolla, K., and Sastry, S., 'Estimation over wireless sensor networks', Proceedings of the 2007 American Control Conference, Marriott Marquis Hotel at Times Square, New York City, USA, July 11-13, 2007, pp. 2732 2737.

[224] Portas, E. B., Orozco, J. A. L., Cruz, J. M., 'Multisensor out-Of-sequence data fusion for estimating the State of dynamic systems', 2007 Information, Decision and Control, pp. 348-353. 
[225] Shalom, B., Y., 'Update with out-of-Sequence measurements in tracking: exact solution', IEEE Transactions on Aerospace and Electronic Systems, vol. 38(3), pp 769-778. Feb. 2002.

[226] Shalom, B., Y., Mallick, M., Chen, H., Washburn, R., 'One step solution for the general out-of-sequence measurement problem in tracking', In: Proc. of the IEEE Aerospace Conference, pp. 1-9. 2002.

[227] Challa, S., Evans, R., Wang, X. 'A Bayesian solution and its approximation to out-of-sequence measurement problems', Information Fusion 4, pp. 185-199, Elservier, 2003.

[228] Zhang, K., Li, R., X., Chen, Y., 'Optimal update with out-of-sequence measurements for distributed filtering', In: Proc. of 5th International Conference of Information Fusion (ISIF2002), pp. 1519-1526. 2002.

[229] Zhang, K., Li, R., X., Chen, Y., 'Multi-sensor multi-tracking with out-ofSequence measurements', In: Proc. of ISIF, 2003. pp. 672-679. 2003.

[230] Orton, M., Marrs, A., 'A Bayesian approach to multi-target tracking and data fusion with out-of-sequence measuerements', In: Proc. of IEE International Workshop on Target Tracking Algorithms and Applications, pp. v1-v15. 2001.

[231] Mookerjee, P., Reifler, F., 'Application of Reduced State Estimation to Multisensor Fusion with Out-Of-Sequence Measurements', In: Proc. of IEEE Radar Conference, pp. 111-116. 2004. 
[232] Oh, S., and Sastry, S., 'Distributed networked control system with lossy links: state estimation and stabilizing communication control', Proceedings of the 45th IEEE Conference on Decision and Control, Manchester Grand Hyatt Hotel, San Diego, CA, USA, December 13-15, 2006, pp. 1942-1947.

[233] Franco, E., Saber, O., R., Parisini, T., and Polycarpou, M. M., 'Distributed fault diagnosis using sensor networks and consensus-based filters', Proceedings of the 45th IEEE Conference on Decision and Control, Manchester Grand Hyatt Hotel, San Diego, CA, USA, December 13-15, 2006, pp. 386-391.

[234] Schenato, L., Sinopoli, B., Sastry, S. S., 'Foundations of control and estimation over lossy networks', Proceedings of the IEEE, vol. 95(1), January 2007, pp. 163187.

[235] Zuo, L., Mehrotra, K., Varshney, P. K., and Mohan, C. K., 'Bandwidth-efficient target tracking In distributed sensor networks using particle filters', 2006 9th International Conference on Information Fusion, 10-13 July 2006, pp. 1-4.

[236] Sheng, X., and Hu, Y. -H., 'Distributed particle filters for wireless sensor network target tracking', In: ICASSP, 2005, Philadelphia, PA, USA.

[237] Ribeiro, A., Giannakis, G. B., and Roumeliotis, S. I., 'SOI-KF: distributed Kalman filtering with low-cost communications Using the sign of innovations', IEEE Transactions on Signal Processing, vol. 54(12), December 2006, pp. 47824795 . 
[238] Ribeiro, A., Giannakis, G. B., and Roumeliotis, S. I., 'SOI-KF: distributed Kalman filtering with low-cost communications using the sign of innovations', In: Proc. Int. Conf. Acoustics, Speech, Signal Processing, Toulouse, France, May 1419, 2006, pp. IV-153-IV-156.

[239] Ma, J., Sun, S., 'Self-Tuning information fusion reduced-Order Kalman predictors for Stochastic Singular Systems', Proceedings of the 6th World Congress on Intelligent Control and Automation, June 21 - 23, 2006, Dalian, China, pp. 1524 1528.

[240] Sun, S. L., 'Multi-sensor information fusion white noise filter weighted by scalars based on Kalman predictor', Automatica, vol. 40(8), pp. 1447-1453, 2004.

[241] Sun, S. L., 'Multi-sensor optimal information fusion input white noise deconvolution estimators', IEEE Trans. Systems, Man, and Cybernetics, vol. 34(4), pp. 1886-1893, 2004.

[242] Spanos, D. P., Olfati-Saber, R., and Murray, R. M., 'Approximate distributed Kalman filtering in sensor networks with quantifiable performance', Fourth International Symposium on Information Processing in Sensor Networks, pp. 133-139, April 2005.

[243] Saber, O., R., Shamma, J. S., 'Consensus filters for sensor networks and distributed Sensor Fusion', Proceedings of the 44th IEEE Conference on Decision and Control, and the European Control Conference 2005, Seville, Spain, December 12-15, 2005, pp. 6698-6703. 
[244] Sun, S., and Zhang, C., 'Optimal information fusion distributed smoother for discrete multichannel ARMA signals', IEE Proc.-Vis. Image Signal Process., vol. 152(5), October 2005, pp. 583-589.

[245] Moir, T. J., and Grimble, M. J., 'Optimal self-tuning filtering, prediction and smoothing for discrete multivarianble processes', IEEE Trans. Autom. Control, 1984, vol. 29(2), pp. 128-137.

[246] Hagander, P., and Wittenmark, B., 'A self-tuning filtering for fixed-lag smoothing', IEEE Trans. Inf. Theory, 1977, vol. 23, pp. 377-384.

[247] Song, E., Zhu, Y., Zhou, J., Sichuan, C., 'The optimality of Kalman filtering fusion with cross-correlated sensor Noises', 43rd IEEE Conference on Decision and Control, 610064, P.R. China, , December 14-17, 2004, Atlantis, Paradise Island, Bahamas, pp. 4637-4642.

[248] Zhu, Y. M., You, Z. S., Zhao, J., Zhang, K. S., and Li, R., X., 'The optimality of the distributed Kalman filter with feedback', Automatica, vol. 37, pp. 1489-1493, 2001.

[249] Zhu, Y., Song, E., Zhou, J., and You, Z., 'Optimal dimensionality reduction of sensor data in multi-sensor estimation fusion', IEEE Transactions on Signal Processing, vol. 53(5), May 2005. pp. 1631-1639.

[250] Sheng, X., and Hu, Y. H., 'Distributed particle filters for wireless sensor network target tracking', ICASSP 2005, pp. IV-845-IV-848. 
[251] Coates, M., 'Distributed particle filters for sensor networks', Information Processing in Sensor Networks, IPSN2003, Springer, pp. 99-107, 2004.

[252] Deng, Z., and Qi, R., 'Multi-sensor infomation fusion sub-optimal steady-state Kalman filter', Chinese Scicnss Absmcts, 2000, vol. 6(2), pp. 183-184.

[253] 'Comparison of two measurement fusion methods for Kalman-filter-Based multisensor data fusion', IEEE Transactions on Aerospace and Electronic Systems, vol. 37(1), January 2001, pp. 273-280.

[254] Chang, K. C., Tian, Z., and Saha, R. K., 'Performance evaluation of track fusion with information filter', In: Proceedings of International Conference on Multisource-Multisensor Information Fusion, July 1998, pp. 648-655.

[255] Saha, R. K., and Chang, K. C., 'An efficient algorithm for multisensor track fusion', IEEE Transactions on Aerospace and Electronic Systems, vol. 34(1), 1998, pp. 200-210.

[256] Saha, R. K., 'Track-to-track fusion with dissimilar sensors', IEEE Transactions on Aerospace and Electronic Systems, vol. 32(3), 1996, pp. 1021-1029.

[257] Whyte, D., H. F., Rao, B. Y. S., and Hu, H., 'Toward a fully decentralized architecture for multi-sensor data fusion', pp, 2/1-2/4.

[258] Zi-Li, D., Bo, C., Li., 'Self-tuning information fusion Kalman predictor weighted by diagonal matrices and its convergence analysis', Acta Automatica Sinica, vol. 33(2), February 2007, pp. 156-163. 
[259] Li, S., S., Jing, M., 'Distributed reduced-order optimal fusion Kalman filters for stochastic singular systems', Acta Automatica Sinica, vol. 32(2), March 2006, pp. 286-290.

[260] Chang, K. C., Zhi, T., Saha, R. K., 'Performace evaluation of track fusion with information matrix filter', IEEE Transactions on Aerospace and Electronic Systems, vol. 38(2), April 2002, pp. 455-466.

[261] Belkin, B., Anderson, S. L., and Sommar, K. M., 'The pseudo-measurement approach to track-to-track data fusion', In: Proceedings of the 1993 Joint Service Data Fusion Symposium, 1993, pp. 519-538.

[262] Drummond, O. E., 'Feedback in track fusion without process noise', In: Proceedings of the SPIE Conference on Signal and Data Processing of Small Targets, vol. 2561, 1995, pp. 369-383.

[263] Liu, Z., Wang, M., Huang, J., 'An evaluation of several fusion algorithms for multi-sensor tracking system', Journal of Information and Computational Science. vol. 7(10), 2010, pp. 2101-2109.

[264] Chang, K. C., Saha, R. K., Bar-Shalom, Y., 'On optimal track-to-track fusion', IEEE Transactions on Aerospace and Electronic Systems, vol. 33, 1997, pp. 12711276.

[265] Shalom, B., Y., 'On the track-to-track cross-covariance problem', IEEE Transactions on Automatic Control, vol. 26, 1981, pp. 571-572. 
[266] Chang, K. C., Tian, Z., Saha, R. K., 'Performance evaluation of track fusion with information matrix filter', IEEE Transactions on Aerospace and Electronic Systems, vol. 38(2), 2002, pp. 455-466.

[267] Abdelgawad, A., and Bayoumi, M., 'Low-power distributed Kalman filter for Wireless Sensor Networks', Hindawi Publishing Corporation EURASIP Journal on Embedded Systems, Volume 2011, Article ID 693150, 11 pages, doi:10.1155/2011/693150.

[268] Kirti, S., and Scaglione, A., 'Scalable distributedkKalman filtering through consensus', In: Proceedings of the IEEE International Conference on Acoustics, Speech and Signal Processing (ICASSP 08), March-April 2008, pp. 2725-2728,

[269] Khan, U. A., and Moura, J. M. F., 'Model distribution for distributed Kalman filters: a graph theoretic approach', In: Proceedings of the 41st Asilomar Conference on Signals, Systems and Computers (ACSSC 07), November 2007, , pp. 611-615.

[270] Azizi, S. M., and Khorasani, K., 'A distributed Kalman filter for actuator fault estimation of deep space formation flying satellites', In: Proceedings of the $3 \mathrm{rd}$ IEEE International Systems Conference, March 2009, pp. 354-359,

[271] Bai, H., and Freeman, R. A., and Lynch, K. M., 'Distributed Kalman filtering using the internal model average consensus estimator', 2011 American Control Conference, O’Farrell Street, San Francisco, CA, USA, June 29 - July 01, 2011, pp. $1500-1505$. 
[272] Cortes, J., 'Distributed kriged Kalman filter for spatial estimation', IEEE Transactions on Automatic Control, vol. 54(12), December 2009, pp. 2816-2827.

[273] Spanos, D. P., Saber, S., R., and Murray, R. M., 'Distributed Kalman filtering in sensor networks with quantifiable performance', In: Information Processing for Sensor Networks, 2005, pp. 133-139.

[274] Qiang, G., Song-nian, Y., 'Distributed multisensor data fusion based on Kalman filtering and the parallel implementation', Journal of Shanghai University ( English Edition ), 2006, vol. 10(2), pp. 118-122, Article ID: 1007-6417(2006)02-0118-05.

[275] Zhu, Y., You, Z., Zhao, J., Zhang, K., and Li, R., X., 'The optimality for the distributed Kalman filtering Fusion', Automatica, vol. 37(9), 2001, pp. 1489-1493.

[276] XiaoJing, S., YingTing, L., YunMin, Z., and EnBin, S., 'Globally optimal distributed Kalman filtering fusion', Science China Information Sciences, March 2012, vol. 55(3): 512529, DOI: 10.1007/s11432-011-4538-7.

[277] Zhu, Y. M., You, Z. S., Zhao, J., et al., 'The optimality for the distributed Kalman filter with feedback', Automatica, 2001, vol. 37, pp. 1489-1493.

[278] Zhao, M. H., Zhu, Z. M., Meng, S., et al., 'Suboptimal distributed Kalman filtering fusion with feedback', J Syst Engin Electron, 2005, vol. 4, pp. 746-749.

[279] Shen, X. J., Luo, Y. T., Zhu, Y. Z., et al., 'Globally optimal flight path update with adding or removing out-of-sequence measurements', Automatica, 2010, vol. 46, pp. 1437-1442. 
[280] Rothrock, R., 'The need to remove out-of-sequence measurements', In: Presented at the Tracking and CID Alternatives Identification and Evaluation Team (TCAIET) Meeting, Arlington, VA, 2006.

[281] Gao, Y., Tao, G. L., Deng, Z. L., 'Decoupled distributed Kalman fuser for descriptor systems', Signal Processing, vol. 88, 2008, pp. 1261-1270.

[282] Lendeka, Z., Babuska, R., Schuttera, B., 'Distributed Kalman filtering for cascaded systems', Engineering Applications of Artificial Intelligence, vol. 21, 2008, pp. $457-469$.

[283] Deng, Z. L., Ma, J. W., and Gao, Y., 'Two-sensor self-tuning information fusion Kalman filter', Science Technology and Engineering , 2003, vol. 3, pp. 321-324.

[284] Sun, S. L., 'Multi-sensor information fusion white noise filter weighted by scalars based on Kalman predictor', Automatica, 2004, vol. 40(8), pp. 1447-1453.

[285] Gao, Y., Ran, C. J., Sun, X. J., and Deng, Z. L., 'Optimal and self-tuning weighted measurement fusion Kalman filters and their asymptotic global optimality', International Journal of Adaptive Control and Signal Processing, Int. J. Adapt. Control Signal Process, 2010, vol. 24, pp. 982-1004.

[286] Hagander, P., Wittenmark, B., 'A self-tuning filter for fixed-lag smoothing', IEEE Transactions on Information Theory, 1977, vol. 23(3), pp. 377-384.

[287] Moir, T., Grimble, M. J., 'Optimal self-tuning filtering, prediction, and smoothing for multivariable processes', IEEE Transactions on Automatic Control, 1984, vol. 29(2), pp. 128-137. 
[288] Deng, Z. L., Zhang H. S., Liu, S. J., Zhou, L., 'Optimal and self-tuning white noise estimators with applications to deconvolution and filtering problems', Automatica, 1996, vol. 32(2), pp. 199-216.

[289] Fung, P. T. K., Grimble, M. J., 'Dynamic ship positioning using a self-tuning Kalman filter', IEEE Transactions on Automatic Control, 1983, vol. 28(3), pp. 339350.

[290] Deng, Z. L., Li, C. B., 'Self-tuning information fusion Kalman predictor weighted by scalars', Proceeding of the Sixth World Congress on Control and Automation, vol. 2, Dalian, China, 2123 June 2006, pp. 1487-1491.

[291] Deng, Z. L., Gao, Y., Li, C. B., Hao, G., 'Self-tuning decoupled information fusion Wiener state component filters and their convergence', Automatica, 2008, vol. 44, pp. 685-695.

[292] Hao, G., Deng, Z. L., 'Self-tuning measurement fusion Kalman filter', Proceeding of the Sixth World Congress on Control and Automation, Dalian, China, vol. 2, 2123 June 2006, pp. 1571-1575.

[293] Hao, G., Jia, W. J., Deng, Z. L., 'Self-tuning multisensor measurement fusion Kalman filter', Proceedings of the 25th Chinese Control Conference, Harbin, China, 711 August, 2006, pp. 395-403.

[294] Gao, Y., Jia, W. J., Sun, X. J., Deng, Z. L., 'Self-tuning multisensor weighted measurement fusion Kalman filter', IEEE Transactions on Aerospace and Electronic Systems, 2009, vol. 45(1), pp. 168-179. 
[295] Fong, L. W., 'Multi-sensor track-to-track fusion via linear minimum variance sense estimators', Asian Journal of Control, vol. 10(3), May 2008, pp. 277-290.

[296] Fong, L. W., 'Integrated track-to-track fusion with modified probabilistic neural network', 2006 CIE Int. Conf. Radar, Shanghai China, 2006, vol. 2, pp. 1869-1873.

[297] Quirino, R. B., and Bottura, C. P., 'An approach for distributed Kalman filtering', SBA Controle and Automacoa, vol. 12(1), 2001, pp. 19-28.

[298] Quirino, R. B., Bottura, C. P., Costa F. J. T., 'A computational structure for parallel and distributed Kalman filtering', Proc. 12th Brazilian Automatic Control Conference, Uberlandia-MG, Brazil, 14-18 de Setembro de 1998.

[299] Spanos, D. P., Saber, O., R., Murray, R. M., 'Distributed Kalman filtering in sensor networks with quantifiable performance', In: 2005 Fourth International Symposium on Information Processing in Sensor Networks (IPSN), 2005, pp. 133-139.

[300] Assimakis, N. D., Tziallas, G., Koutsonikolas, A., 'Optimal distributed Kalman filter', Technical Report, pp. 1-19.

[301] Mallick, M., Kirubarajan, T., and Arulampalam, S., 'Out-of-sequence measurement processing for tracking ground target using particle flters', In: Proceedings of the IEEE Aerospace Conference, Big Sky, MT, USA, vol. 4, March 2002, pp. 1809-1818.

[302] Mallick, M., and Marrs, A., 'Comparison of the KF and particle flter based outof-sequence measurement fltering algorithms', In: Proceedings of the Sixth Inter- 
national Conference on Information Fusion, Cairns, Qld., Australia, vol. 1, July 2003, pp. 422-429.

[303] Shalom, B., Y., and Campo, L., 'The effect of the common process noise on the two-sensor fused-track covariance', IEEE Transactions on Aerospace and Electronic Systems, vol. AES-22, 1986, pp. 803-805.

[304] Chong, C. Y., Mori, S., and Chang, K. C., 'Information fusion in distributed sensor networks', In: Proc. of the American Control Conference, 1985, pp. 830835.

[305] Mori, S., Barker, W. H., Chong, C. Y., and Chang, K. C., 'Track association and track fusion with non-deterministic target dynamics', IEEE Transactions on Aerospace and Electronic Systems, vol. AES-38, 2002, pp. 659-668.

[306] Roecker, J. A., and McGillem, C. D., 'Comparison of two-sensor tracking methods based on state vector fusion and measurement fusion', IEEE Transactions on Aerospace and Electronic Systems, vol. AES-24, 1988, pp. 447-449.

[307] Chou, K. C., Willsky, A. S., Benveniste, A., 'Multiscale recursive estimation, data fusion, and regularization', IEEE Transactions on Automatic Control, vol. 39(3), March 1994, pp. 464-474.

[308] Ran, C. J., Tao, G. L., Liu, J. F., Deng, Z. L., 'Self-tuning decoupled fusion Kalman predictor and its convergence analysis', IEEE Sensors Journal, 2009, vol. 9(12), pp. 2024-2032. 
[309] Sinopoli, B., Schenato, L., Franceschetti, M., Poolla, K., Jordan, M. and Sastry, S., 'Kalman filtering with intermittent observations', IEEE Trans. Automatic Control, vol. 49(9), pp. 1453-1464, 2004.

[310] Liu, X., and Goldsmith, A., 'Kalman filtering with partial observation losses', In Proc. of the 43rd IEEE Conference on Decision and Control, Paradise Island, Bahamas, Dec. 2004.

[311] Figueiredo, M. A. T., and Jain, A. K., 'Unsupervised learning of finite mixture models', IEEE Trans. Pattern Anal. Mach. Intell., vol. 24(3), pp. 381-396, Mar. 2002.

[312] Ren, W., and Beard, R. W., 'Consensus seeking in multiagent systems under dynamically changing interaction topologies', IEEE Trans. Autom. Control, vol. 50(5), pp. 655-661, May 2005.

[313] Ding, D., Wang, Z., Dong, H., and Shu, H., 'Distributed H-infinity state estimation with stochastic parameters and nonlinearities through sensor networks: the finite-horizon case', Automatica, vol. 48(8), Aug. 2012, pp. 1575-1585.

[314] Dong, H., Wang, Z., and Gao, H., 'Distributed filtering for a class of timevarying systems over sensor networks with quantization errors and successive packet dropouts', IEEE Transactions on Signal Processing, vol. 60, No. 6, Jun. 2012, pp. 3164-3173.

[315] Liang, J., Wang, Z., and Liu, X., 'Distributed state estimation for uncertain Markov-type sensor networks with mode-dependent distributed delays', Interna- 
tional Journal of Robust and Nonlinear Control, vol. 22(3), Feb. 2012, pp. 331346.

[316] Shen, B., Wang, Z., and Liu, X., 'A stochastic sampled-data approach to distributed H-infinity filtering in sensor networks', IEEE Transactions on Circuits and Systems - Part I, vol. 58(9), Sept. 2011, pp. 2237-2246.

[317] Shen, B., Wang, Z., Hung, Y. S., and Chesi, G., 'Distributed H-infinity filtering for polynomial nonlinear stochastic systems in sensor networks', IEEE Transactions on Industrial Electronics, vol. 58(5), May 2011, pp. 1971-1979.

[318] Liang, J., Wang, Z., and Liu, X., 'Distributed state estimation for discrete-time sensor networks with randomly varying nonlinearities and missing measurements', IEEE Transactions on Neural Networks, vol. 22(3), Mar. 2011, pp. 486-496.

[319] He, X., Wang, Z., Ji, Y., and Zhou, D., 'Robust fault detection for networked systems with distributed sensors', IEEE Transactions on Aerospace and Electronic Systems, vol. 47(1), Jan. 2011, pp. 166-177.

[320] Shen, B., Wang, Z., and Hung, Y. S., 'Distributed consensus H-infinity filtering in sensor networks with multiple missing measurements: the finite-horizon case', Automatica, vol. 46(10), Oct. 2010, pp. 1682-1688.

[321] Deutscher, J., and C. Harkort, C., 'Parametric approach to the decoupling of linear distributed-parameter systems', IET Control Theory Appl., December 2010, vol. 4(12), pp. 2855-2866. 
[322] Li, J., Xu, S., Chu, Y., and Wang, H., 'Distributed average consensus control in networks of agents using outdated states', IET Control Theory Appl., May 2010, vol. 4(5), pp. 746-758.

[323] Smith, R. S., and Hadaegh, F. Y., 'Distributed estimation, communication and control for deep space formations', IET Control Theory Appl., March 2007, vol. 1(2), pp. 445-451.

[324] Azizi, S. M., Tousi, M. M., and Khorasani, K., 'Multi-agent methodology for distributed and cooperative supervisory estimation subject to unreliable information', IET Control Theory Appl., March 2011, vol. 5(4), pp. 606-621.

[325] Wen, K., and Geng, Z., 'Modelling and analysis of distributed networked control systems', IET Control Theory Appl., June 2012, vol. 6(9), pp. 1304-1312.

[326] Yang, S., and Zhao, Q., 'Probability distribution characterisation of fault detection delays and false alarms', IET Control Theory Appl., May 2012, vol. 6(7), pp. 953-962.

[327] Ugrinovskii, V., and Langbort, C., 'Distributed $H_{\infty}$ consensus-based estimation of uncertain systems via dissipativity theory', IET Control Theory Appl., August 2011, vol. 5(12), pp. 1458-1469.

[328] Li, W., and Jia, Y., 'Consensus-based distributed information filter for a class of jump Markov systems', IET Control Theory Appl., July 2011, vol. 5(10), pp. 1214-1222. 
[329] Chen, Z., 'Bayesian filtering: From Kalman filters to particle filters and beyond', adaptive Syst Lab McMaster Univ Hamilton ON Canada, Citeseer, 2003, pp. 9-13, 25-46.

[330] Oh, S., and Sastry, S., 'Approximate estimation of distributed networked control system', 2007 ACC '07 American Control Conference, 9-13 July 2007, pp. 9971002.

[331] Rao, B. S., and Whyte, H. F., 'Fully decentralised algorithm for multisensor kalman filtering', IEEE Proceedings-D, 1991.

[332] Li, S., S., 'Optimal multi-sensor kalman smoothing fusion for discrete multichannel ARMA signals', Journal of Control Theory and Applications, 2005, vol. 2, pp. $168-172$.

[333] Sun, S., 'Multi-sensor optimal information fusion Kalman filter for discrete multichannel ARMA signals', Proc. of 2003 IEEE Int. Symposium on Intelligent Control, Chicago : Institute of Electrical and Electronic Engineers Inc., Houston, October 2003 , pp. $377-382$.

[334] Sun, S., Deng, Z., 'Multi-sensor optimal information fusion Kalman filter', $A u$ tomatica, 2004, vol. 40(6), pp. 1017-1023.

[335] Luo, Y., Zhu, Y., Luo, D., Zhou, J., Song, E., and Wang, D., 'Globally optimal multisensor distributed random parameter matrices kalman filtering fusion with applications', Sensors 2008, vol. 8, pp. 8086-8103; DOI: 10.3390/s8128086. 
[336] Song, E. B., Zhu, Y. M., Zhou, J., You, Z. S., 'Optimality kalman filtering fusion with cross-correlated sensor noises', Automatica 2007, vol. 43, pp. 1450-1456.

[337] Rao, B. S., Whyte, D-, H. F., 'Fully decentralized algorithm for multisensor kalman filtering', IEE Proceedings on Control Theory and Applications, Sep. 1991; Part D, vol. 138, pp. 413-420.

[338] Chong, C. Y., Chang, K. C., Mori, S., 'Distributed tracking in distributed sensor networks', Proceedings of the American Control Conference, Seattle, 1986.

[339] Hashmipour, H. R., Roy, S., Laub, A. J., 'Decentralized structures for parallel kalman filtering', IEEE Trans. Autom. Control, 1988, vol. 33, pp. 88-93.

[340] Saber, R. O., and Murray, R. M., 'Consensus problems in networks of agents with switching topology and time-delays', IEEE Trans. on Automatic Control, vol. 49(9), pp. 1520-1533, Sep. 2004.

[341] Saber, R. O., and Murray, R. M., 'Consensus protocols for networks of dynamic agents', Proc. of the American Control Conference, vol. 2, pp. 951-956, June 2003.

[342] Mesbahi, M., 'On state-dependent dynamic graphs and their controllability properties', IEEE Trans. on Automatic Control, vol. 50(3), pp. 387-392, 2005.

[343] Saber, R. O., 'Ultrafast consensus in small-world networks', Proceedings of American Control Conference, June 2005.

[344] Hatano, Y., and Mesbahi, M., 'Agreement over random networks', IEEE Conf. on Decision and Control, 2004. 
[345] Moreau, L., 'Stability of multiagent systems with time-dependent communication links', IEEE Trans. on Automatic Control, vol. 50(2), pp. 169-182, 2005.

[346] Ren, W., and Beard, R. W., 'Consensus seeking in multi-agent systems under dynamically changing interaction topologies', IEEE Trans. on Automatic Control, vol. 50(5), pp. 655-661, 2005.

[347] Xiao, L., and Boyd, S., 'Fast linear iterations for distributed averaging', Systems and Control Letters, vol. 52, pp. 65-78, 2004.

[348] Xiao, L., Boyd, S., and Lall, S., 'A scheme for asynchronuous distributed sensor fusion based on average consensus', Fourth International Symposium on Information Processing in Sensor Networks, April 2005.

[349] Saber, O. R., 'Flocking for multi-agent dynamic systems: algorithms and theory', IEEE Transactions on Automatic Control, vol. 51(3), pp. 401-420.

[350] Zhu, Y. M., You, Z. S., Zhou, J., Zhang, K. -S., and Li, X. R., 'The optimality for the distributed Kalman filtering fusion with feedback', Automatica, vol. 37, pp. 1489-1493, 2001.

[351] Sheng, Y., Hu, X., and Ramanathan, P., 'Distributed particle filter with GMM approximation for multiple targets localization and tracking in wireless sensor networks', Fourth International Symposium on Information Processing in Sensor Networks, April 2005.

[352] Whyte, H. D., 'Data fusion in sensor networks', Fourth International Symposium on Information Processing in Sensor Networks, April 2005. 
[353] Grime, S., and Whyte, H. D., 'Data fusion in decentralized sensor networks', J. Control Engineering Practice, vol. 2, 1994.

[354] Spanos, D. P., Saber, R. O., and Murray, R. M., 'Distributed sensor fusion using dynamic consensus', Proceedings of IFAC World Congress, 2005.

[355] Costa, O., and Fragoso, M., 'Stability results for discrete-time linear systems with Markovian jumping parameters', Journal of Mathematical Analysis and Applications, vol. 179, pp. 154-178, 1993.

[356] Mosca, E., 'Optimal, predictive, adaptive control', New Jersey: Prentice-Hall, 1995.

[357] Mahmoud, M. S., and Emzir, M. F., 'State estimation with asynchronous multirate multi-smart sensors', Information Sciences, volume 196(1), August 2012, pp. $15-27$.

[358] Kaddissi, C., Kenne, J. -P., and Saad, M., 'Identification and real time control of an electro-hydraulic servo system based on nonlinear backstepping', IEEE/ASME Transactions on Mechatronics, vol. 12, 2007, pp. 12-22

[359] Sukhavasi, R. T., Hassibi, B.,'The Kalman like particle filter: Optimal estimation with quantized innovations/measurements', CDC, 2009, pp. 4446-4451.

[360] Martinez, J. J., Landau, I. D., Buche, G., 'Design and tuning of reduced order Hinfinity feedforward compensators for active vibration control',IEEE Transactions on Control Systems Technology, vol. 20(2), March 2012, pp. 554-561. 
[361] Chenier, F., Bigras, P., Aissaoui, R., 'An orientation estimator for the wheelchair's caster wheels', IEEE Transactions on Control Systems Technology, November 2011, vol. 19(6), pp. 1317-1326.

[362] Smith, K. A., Rahn, C. D., Wang, C. -Y., 'Model-based electrochemical estimation and constraint management for pulse operation of lithium ion batteries', IEEE Transactions on Control Systems Technology, May 2010, vol. 18(3), pp. 654-663.

[363] Lightcap, C. A., Banks, S. A., 'An extended kalman filter for real-time estimation and control of a rigid-link flexible-joint manipulator', IEEE Transactions on Control Systems Technology, January 2010, vol. 18(1), pp. 91-103.

[364] Zhou, D. H., He, X., Wang, Z., Liu, G. -P., Ji, Y. D., 'Leakage fault diagnosis for an internet-based three-tank system: An experimental study', IEEE Transactions on Control Systems Technology, July 2012, vol. 20(4), pp. 857-870.

[365] Meskin, N., Khorasani, K., Rabbath, C. A., 'A hybrid fault detection and isolation strategy for a network of unmanned vehicles in presence of large environmental disturbances', IEEE Transactions on Control Systems Technology, November 2010, vol. 18(6), pp. 1422-1429.

[366] Kalman, R. E., 'A new approach to linear filter and prediction problems', Journal of Basic Engineering, vol. 82, pp. 35-45.

[367] Anderson, B. D. O., Moore, J. B., 'Optimal filtering', Prentice Hall, 1979, 357 pp.

[368] Simon, D., 'Optimal state estimation', Wiley and Sons, 2006, 530 pp. 
[369] Cohn, S. E., Sivakumaran, N. S., Todling, R., 'A fix-lag Kalman smoother for data assimilation', Monthly Weather review, vol. 122, pp. 2838-2867.

[370] Evensen, G., Leeuwen, P. J., 'An ensemble Kalman smoother for nonlinear dynamics', Monthly Weather review, vol. 128, pp. 1852-1867.

[371] Li, S. S., and Jing, M. A., 'Distributed reduced-order optimal fusion kalman filters for stochastic singular systems', Acta Automatica Sinica, March 2006, vol. 32(2), pp. 286-290.

[372] Cosme, E., Brankart, J. -M., Verron, J., Brasseur, P., Krysta, M., 'Implementation of a reduced rank square-root smoother for high resolution ocean data assimilation', Ocean Modelling, vol. 33, 2010, pp. 87-100.

[373] Deng, Z. L., 'Kalman filtering and Wiener filtering - Modern time series analysis method', Harbin, Harbin Institute of Technology, 2001, pp. 1-396.

[374] Khan, M. E., and Dutt, D. N., 'An expectation maximization algorithm based on Kalman smoother approach for event-related desynchronization (ERD) estimation from EEG', em IEEE Trans. on Biomedical Engg., July 2007, vol. 54(7), pp. 11911198.

[375] Mahmoud, M. S., and Khalid, H. M., 'Expectation maximization approach to data-based fault diagnostics', Elsevier-Information Sciences - Special Issue, Available online, January 2012, DOI: 10.1016/j.ins.2012.01.031.

[376] Moon, T. K., 'The expectation maximization algorithm', IEEE Signal Processing Magazine, November 1996, pp. 47-59. 
[377] Pham, D., Verron, J., Roubaud, M., 'A singular evolutive extended Kalman filter for data assimilation of sea-surface temperature and altimetric data in the southatlantic ocean', J. Marine Res., 2002, vol. 60, pp. 805-833.

[378] Cohn, S. E., Todling, R., 'Approximate Kalman filters for unstable dynamics', Proceedings of the Second International Symposium on assimilation of observations in meteorology and oceanography, WMO, 1995, pp. 241-246.

[379] Cohn, S. E., Todling, R., 'Approximate Kalman filters for stable and unstable dynamics', J Meteor. Soc., Japan, vol. 74, 1996, pp. 63-75.

[380] Verlaan, M., and Heemink, A. W., 'Reduced rank square root filters for large scale data assimilation problem', Proceedings of the Second International Sysmposium on Assimilation of Observations in Meteorology and Oceanography, WMO, 1995, pp. 247-252.

[381] Rao, B. S., and Whyte, H. F., 'Fully decentralised algorithm for multisensor kalman filtering', IEEE Proceedings-D, 1991.

[382] Idkhajine, L., Monmasson, E., Maaalouf, A.,'Fully FPGA-based sensorless control for synchronous AC drive using an extended Kalman filter', IEEE Trans. on Industrial Electronics, October 2012, vol. 59(10), pp. 3908-3915.

[383] Yen, W., and Hua, T. L., 'A high-performance sensorless position control system of a synchronous reluctance motor using dual current-slope estimating technique', IEEE Trans. on Industrial Electronics, September 2012, vol. 59(9), pp. 3411-3426. 
[384] Rigatos, G., 'A derivative-free Kalman filtering approach to state estimationbased control of nonlinear systems', IEEE Trans. on Industrial Electronics, October 2012, vol. 59(10), pp. 3987-3997.

[385] Luo, R., and Lai, C., 'Enriched indoor map construction based on multisensor fusion approach for intelligent service robot', IEEE Trans. on Industrial Electronics, August 2012, vol. 59(8), pp. 3135-3145.

[386] Sadinezhad, I., and Agelidis, V., 'Frequency adaptive least-squares-Kalman technique for real-time voltage envelope and flicker estimation', IEEE Trans. on Industrial Electronics, August 2012, vol. 59(8), pp. 3330-3341.

[387] Shi, P., Luan, X., and Liu, F., ' $H_{\infty}$ filtering for discrete-time systems with stochastic incomplete measurement and mixed delays', IEEE Trans. on Industrial Electronics, June 2012, vol. 59(6), pp. 2732-2739.

[388] Shalom, Y., 'On the track-to-track correlation problem', IEEE Trans on AC, 1981, vol. 26(2), pp. 571-572.

[389] Shalom, Y., and Campo, L., 'The effect of the common process noise on the twosensor fused-track covariance', IEEE Trans On AES, 1986, AES-22, pp. 803-805.

[390] Chang, K. C., Saha, R. K., and Shalom Y., 'On optimal track-to-track fusion. IEEE Trans On AES, 1997, vol. 33(4): 1271-1276.

[391] Chong, C. Y., Chang K. C., and Mori, S., 'Distributed tracking in distributed sensor networks', Proceedings of the American Control Conference, Seattle, 1986. 
[392] Julier, S., and Uhlmann, J. K., 'General decentralized data fusion with Covariance Intersection (CI)', In: D. Hall and J. Llinas, editors, Handbook of multisensor data fusion, chapter12, pp. 12-1-12-25. CRC Press, 2001.

[393] Li, X. R., Zhu, Y. M., and Han, C. Z., 'Unified optimal linear estimation fusion Part I: unified model and fusion rules', Proceedings of the 3rd International Information Fusion Conference, Paris, France: ISIF. 2000, pp. MoC2-10-Moc2-17.

[394] Chang K. C., Zhi, T., and Saha, R. K., 'Performance evaluation of track fusion with information matrix filter', IEEE Trans On AES, 2002, vol. 38(2), pp. 455-466.

[395] Chang, K. C., 'Evaluating hierarchical fusion with information matrix filter', Proceedings of the 3rd International Information Fusion Conference, Paris, France: ISIF. 2000.

[396] Chong, C. Y., and Mori, S., 'Convex combination and covariance intersection algorithm in distributed fusion', Proceedings of the 4th International Information Fusion Conference, Montreal, Canada: ISIF. 2001.

[397] Shalom, B. Y., Campo, L., 'The effect of common process noise on the twosensor fused-track covariance', IEEE Transactions on Aerospace and Electronic Systems, vol. 22, 1986, pp. 803-805.

[398] Sun, S. L., 'Multi-sensor optimal information fusion kalman filter for discrete multichannel ARMA Signals', Proceedings of 2003 IEEE International Symposium on Intelligent Control, 2003, pp. 377-382. 
[399] Sun, S. L., Deng, Z. L., 'Multi-sensor optimal information fusion kalman filter', Automatica, vol. 40, 2004, pp. 1017-1023.

[400] Scala, B. F., Farina, A., 'Choosing a track association method', Information Fusion, vol. 3, 2001, pp. 119-133.

[401] Chong, C. Y., Chang, K. C., Mori, S., 'Distributed tracking in distributed sensor networks', Proceedings of the American Control Conference, Seattle, 1986, pp. $1863-1868$.

[402] Uhlmann, J. K., Julier, S., Whyte, H. F., 'A culminating theory in the theory and practice of data fusion, filtering and decentralized estimation', technical report, Covariance Intersection Working Group, 1997.

[403] Julier, S. J., Uhlman, J. K., 'A non-divergent estimation algorithm in the presence of unknown correlaiions', Proceedings of the American Control Conference, 1997, pp. 2369-2373.

[404] Roecker, J. A., McGillem, C. D., 'Comparison of two-sensor tracking methods based on state vector fusion and measurement fusion', IEEE Transactions on Aerospace and Electronic Systems, vol. 24, 1988, pp. 447-449.

[405] Chang, K. C., Tian, Z., Saha, R. K., 'Performance evaluation of track fusion with information matrix filter', IEEE Transactions on Aerospace and Electronic Systems, vol. 38, 2002, pp. 455-466.

[406] Chang, K. C., Saha, R. K., Shalom, Y., 'On optimal track-to-track fusion’, IEEE Transactions on Aerospace and Electronic Systems, vol. 33, 1997, pp. 1271-1276. 
[407] Shalom, Y., 'On the track-to-track cross-covariance problem', IEEE Transactions on Automatic Control, vol. 26, 1981, pp. 571-572.

[408] Shalom, Y., Campo, L., 'The effect of common process noise on the two-sensor fused-track covariance', IEEE Transactions on Aerospace and Electronic Systems, vol. 22, 1986, pp. 803-805.

[409] Juliera, S., and Uhlmann, J., 'Using covariance intersection for SLAM Robotics and Autonomous Systems', vol. 55, 2007, pp. 3-20.

[410] Dong, Q. X., and Chang, K. C., 'Information matrix fusion with feedback versus number of sensors', Proc. Fusion, 2004, vol. I, pp. 686-692, Stockholm, Sweden, July, 2004.

[411] Gan, Q., Harris, C. J., 'Comparison of two measurement fusion methods for Kalman-filter-based multisensor data fusion', IEEE Transactions on Aerospace and Electronic Systems, January 2001, vol. 37(1), pp. 273-279.

[412] Liu, Z., Wang, M., and Huang, J., 'An evaluation of several fusion algorithms multi-sensor tracking system', Journal of Information and Computational Science, vol. 7(10), 2010, pp. 2101-2109.

[413] Sayed, A. H., 'Fundamentals of Adaptive Filtering', Wiley, NJ, 2003, IEEE Press, 2003.

[414] Sukhavasi, R. T., Hassibi, B., 'The Kalman like particle filter: Optimal estimation with quantized innovations/measurements', $C D C, 2009$, pp. 4446-4451. 
[415] Marquez, H., Riaz, M., 'Robust state observer design with application to an industrial boiler system', Control Engineering Practice, vol. 13, 2005, pp. 713728. 


\section{Vitae}

- Haris M. Khalid

- Received Bachelors of Science (B.S.) degree in Mechatronics and Control Engineering from University of Engineering and Technology, Lahore, Pakistan in 2007.

- Received Masters of Science (M.S.) degree in Control Systems Engineering from King Fahd University of Petroleum and Minerals, Dhahran, Saudi Arabia in 2009.

- Completed Doctor of Philosophy (PhD) degree in Control Systems Engineering in September 2012.

- Email: harism.khalid@yahoo.com 